\title{
TANGLE FLOER HOMOLOGY AND COBORDISMS BETWEEN TANGLES
}

\author{
AKRAM ALISHAHI AND EAMAN EFTEKHARY
}

\begin{abstract}
We introduce a generalization of oriented tangles, which are still called tangles, so that they are in one-to-one correspondence with the sutured manifolds. We define cobordisms between sutured manifolds (tangles) by generalizing cobordisms between oriented tangles. For every commutative algebra $\mathbb{A}$ over $\mathbb{Z} / 2 \mathbb{Z}$, we define $\mathbb{A}$-Tangles to be the category consisting of $\mathbb{A}$-tangles, which are balanced tangles with $\mathbb{A}$-colorings of the tangle strands and fixed $\operatorname{Spin}^{c}$ structures, and $\mathbb{A}$-cobordisms as morphisms. An $\mathbb{A}$-cobordism is a cobordism with a compatible $\mathbb{A}$-coloring and an affine set of $\operatorname{Spin}^{c}$ structures. Associated with every $\mathbb{A}$-module $\mathbb{M}$ we construct a functor

$$
\mathrm{HF}^{\mathbb{M}}: \mathbb{A} \text {-Tangles } \longrightarrow \mathbb{A} \text {-Modules, }
$$

called the tangle Floer homology functor, where $\mathbb{A}$-Modules denotes the the category of $\mathbb{A}$-modules and $\mathbb{A}$-homomorphisms between them. Moreover, for any $\mathbb{A}$-tangle $\mathcal{T}$ the $\mathbb{A}$-module $\mathrm{HF}^{\mathbb{M}}(\mathcal{T})$ is the extension of sutured Floer homology defined in an earlier work of the authors.

In particular, this construction generalizes the 4-manifold invariants of Ozsváth and Szabó. Moreover, applying the above machinery to decorated cobordisms between links, we get functorial maps on link Floer homology.
\end{abstract}

\section{Contents}

1. Introduction

1.1. Introduction and background 2

1.2. Main results 4

1.3. Examples and applications 6

1.4. Outline of the paper $\quad 7$

2. Tangles, Spin $^{c}$ structures and Heegaard poly-tuples 8

2.1. Sutured manifolds and tangles 8

2.2. Heegaard Floer homology for tangles 8

2.3. $\mathrm{Spin}^{c}$ structures over cobordisms 9

2.4. Heegaard poly-tuples 10

2.5. A-diagrams and holomorphic polygon maps 11

3. Tangle complex and the issue of naturality 13

3.1. Oriented graph of isotopy Heegaard diagrams

3.2. Special Heegaard diagrams

3.3. Weak Heegaard invariance 14

3.4. Strong Heegaard invariance 16

3.5. The action of $\Lambda^{*}\left(H_{1}(M, \mathbb{Z}) /\right.$ Tors $) \quad 18$

4. Parametrized Cerf decomposition $\quad 19$

4.1. Parametrized elementary cobordism $\quad 19$

4.2. Parametrized Morse data $\quad 20$

4.3. Cerf moves 23

4.4. Parametrized Cerf decomposition theorem 26

4.5. Ordered Morse data and parametrized decompositions 33

5. One-handles, Three-handles and the cobordism maps 35

5.1. Adding one-handles 35

5.2. Stretching the necks in Heegaard diagrams 35

5.3. Construction of the cobordism map 37

5.4. Proof of Lemma $5.3 \quad 38$ 
5.5. Invariance of the cobordism map for one-handles

5.6. Three-handles

6. Framed arcs, framed knots and cobordism maps 42

6.1. Special tangles corresponding to cobordisms 42

6.2. The distinguished generator 44

6.3. Framed arcs, framed knots and the cobordism map 47

6.4. Proof of Theorem 6.9 51

7. The cobordism map and its invariance 55

7.1. Invariance under arc slides.

7.2. A composition law for framed arcs and links

7.3. The composition law 58

7.4. Action of $\Lambda^{*}\left(H_{1}(W, \mathbb{Z}) /\right.$ Tors $)$

7.5. Relative $\operatorname{Spin}^{c}$ structures and the cobordism map 62

8. Applications and special cases 64

8.1. Cobordisms between closed 3-manifolds 64

8.2. Functoriality of link Floer homology 64

References $\quad 66$

\section{INTRODUCTION}

1.1. Introduction and background. Ozsváth and Szabó introduced Heegaard Floer homology for closed three dimensional manifolds [OS04c, OS04b] which resulted in powerful tools for the study of various structures in low dimensional topology, including invariants for knots OS04a, Ras02, Eft05], for links [OS08, for contact structures OS05] and for sutured manifolds [Juh06, AE15]. Juhász and Thurston [JT] showed that the Heegaard Floer groups associated with three-dimensional objects (closed manifolds, links and sutured manifolds)are in fact functors which associate a concrete module to any of the aforementioned topological objects, rather than just the isomorphism class of it. Typically, Heegaard Floer homology groups come in different flavours, which are denoted by $\widehat{\mathrm{HF}}, \mathrm{HF}^{+}, \mathrm{HF}^{-}$and $\mathrm{HF}^{\infty}$, besides many other flavours which appear in knot and link Floer homology theories. The simplest version of these invariants, $\widehat{\mathrm{HF}}$ and $\widehat{\mathrm{HFK}}$, has been generalized to compact, non-closed 3-manifolds, with a specific on the boundary, called sutured manifolds by Juhász. The authors gave a framework that generalizes sutured Floer homology and brings all flavours of Heegaard Floer homology under the same roof in AE15.

In this paper, to define a natural notion of cobordism between sutured manifolds, that generalizes cobordisms between 3-manifolds, knots and links, we introduce a generalization of classical oriented tangles such that they are in one-to-one correspondence with sutured manifolds without toroidal sutures. So, we call our Heegaard Floer invariants, tangle Floer homology, and denote it by HF.

Definition 1.1. A tangle $(M, T)$ is an oriented 3-manifold $M$ with boundary and a properly embedded, oriented 1-manifold $T$. Both $M$ and $T$ have no closed components, $\partial M$ is equipped with a fixed decomposition $\partial M=\partial^{+} M \amalg \partial^{-} M$ such that $\partial^{-} T \subset \partial^{-} M$ and $\partial^{+} T \subset \partial^{+} M$.

The tangle $(M, T)$ is called balanced if every component of $\partial M$ intersects $T$ and for every connected component $M_{\circ}$ of $M, \chi\left(\partial^{+} M_{\circ}\right)=\chi\left(\partial^{-} M_{\circ}\right)$.

Fix an algebra $\mathbb{A}$ over $\mathbb{F}=\mathbb{Z} / 2 \mathbb{Z}$ (which will always be commutative through this paper). For every connected component $s \in \pi_{0}\left(\partial^{\circ} M\right)$ and any map $\mathfrak{u}: \pi_{0}(T) \rightarrow \mathbb{A}$ define

$$
\mathfrak{u}(s):=\prod_{\substack{t \in \pi_{0}(T) \\ \imath_{T}^{\circ}(t)=s}} \mathfrak{u}(t)
$$

where $\circ=+,-$ and $\imath_{T}^{\circ}: \pi_{0}(T)=\pi_{0}\left(\partial^{\circ} T\right) \rightarrow \pi_{0}\left(\partial^{\circ} M\right)$ is the map induced by the inclusion $\partial^{\circ} T \subset \partial^{\circ} M$. 
Definition 1.2. An $\mathbb{A}$-coloring for a balanced tangle $(M, T)$ is a map $\mathfrak{u}: \pi_{0}(T) \rightarrow \mathbb{A}$ satisfying the following two conditions.

(1) If $s \in \pi_{0}(\partial M)$ corresponds to a connected component with positive genus, then $\mathfrak{u}(s)=0$,

(2) For every connected component $M_{\circ}$ of $M$

$$
\sum_{s \in \pi_{0}\left(\partial^{+} M_{\circ}\right)} \mathfrak{u}(s)=\sum_{s \in \pi_{0}\left(\partial^{-} M_{\circ}\right)} \mathfrak{u}(s)
$$

In particular, $\mathbb{A}=\mathbb{F}$ and $\mathfrak{u}=0$ give an $\mathbb{A}$-coloring for any balanced tangle.

The set $\operatorname{Spin}^{c}(M)$ of $\operatorname{Spin}^{c}$ structures over a tangle $(M, T)$ is the set of homology classes of nonzero vector fields on $M$ which restrict to the outward normal of $\partial^{+} M$ and the inward normal of $\partial^{-} M$.

Definition 1.3. An $\mathbb{A}$-tangle is a 4 -tuple $\mathcal{T}=[M, T, \mathfrak{s}, \mathfrak{u}]$ where $(M, T)$ is a balanced tangle, $\mathfrak{s} \in \operatorname{Spin}^{c}(M)$ is a $\operatorname{Spin}^{c}$ structure over $M$ and $\mathfrak{u}: \pi_{0}(T) \rightarrow \mathbb{A}$ is an $\mathbb{A}$-coloring of $(M, T)$.

If $\mathcal{T}$ is an $\mathbb{A}$-tangle, we use $\left[M_{\mathcal{T}}, T_{\mathcal{T}}, \mathfrak{s}_{\mathcal{T}}, \mathfrak{u}_{\mathcal{T}}\right]$ to denote the corresponding 4-tuple. Given an $\mathbb{A}$ module $\mathbb{M}$ and associated with an $\mathbb{A}$-tangle $\mathcal{T}$ the construction of the authors in [AE15] defines an A-module

$$
\operatorname{HF}^{\mathbb{M}}(\mathcal{T})=H_{*}\left(\mathrm{CF}(\mathcal{T}) \otimes_{\mathbb{A}} \mathbb{M}\right)
$$

so that its isomorphism type is an invariant of $\mathcal{T}$. In light of Juhász and Thurston's naturality discussions in [JT, one can strengthen this result as follows.

Definition 1.4. The category $\mathbb{A}$-Tang is defined such that:

(1) Objects are $\mathbb{A}$-tangles.

(2) Morphisms from an $\mathbb{A}$-tangle $\mathcal{T}=[M, T, \mathfrak{s}, \mathfrak{u}]$ to another $\mathbb{A}$-tangle $\mathcal{T}^{\prime}=\left[M^{\prime}, T^{\prime}, \mathfrak{s}^{\prime}, \mathfrak{u}^{\prime}\right]$ are the diffeomorphisms $d:(M, T) \rightarrow\left(M^{\prime}, T^{\prime}\right)$ such that $d^{*} \mathfrak{s}^{\prime}=\mathfrak{s}$ and the following diagram is commutative.

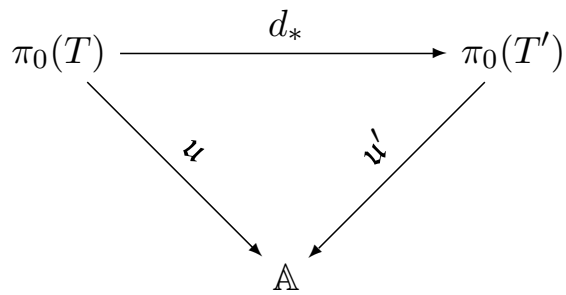

Here $d^{*}: \operatorname{Spin}^{c}\left(M^{\prime}\right) \rightarrow \operatorname{Spin}^{c}(M)$ and $d_{*}: \pi_{0}(T) \rightarrow \pi_{0}\left(T^{\prime}\right)$ are the maps induced by the diffeomorphism $d$.

Let $\mathbb{A}$-Mod denote the category of $\mathbb{A}$-modules together with the isomorphisms between them. Using [JT, Theorem 2.39] one can prove the following theorem (see Section 3).

Theorem 1.1. For every algebra $\mathbb{A}$ over $\mathbb{F}$ and every $\mathbb{A}$-module $\mathbb{M}$, assigning the $\mathbb{A}$-module $\mathrm{HF}^{\mathbb{M}}(\mathcal{T})$ to the $\mathbb{A}$-tangle $\mathcal{T}$ in $\mathbb{A}$-Tang gives a functor

$$
\mathrm{HF}^{\mathbb{M}}: \mathbb{A} \text {-Tang } \longrightarrow \mathbb{A} \text {-Mod. }
$$

Example 1.1. Tangle Floer homology extends sutured Floer homology, as well as different versions of knot and link Floer homology in the following sense:

(a) Let $\mathbb{A}=\mathbb{F}$. Equipping any balanced tangle $(M, T)$ with the trivial $\mathbb{A}$-coloring $\mathfrak{u}=0$ and an arbitrary $\operatorname{Spin}^{c}$ class $\mathfrak{s} \in \operatorname{Spin}^{c}(M)$, we have $\operatorname{HF}^{\mathbb{F}}(M, T, \mathfrak{s}, \mathfrak{u})=\operatorname{SFH}(M, T, \mathfrak{s})$.

(b) Corresponding to any closed, oriented 3-manifold $Y$ with a based point $p \in Y$, there is a tangle $\left(Y_{p}, T_{p}\right)$ where $Y_{p}$ is obtained from $Y$ by removing two disjoint 3-balls, and $T_{p} \subset$ $Y_{p}$ is a properly embedded, oriented arc connecting the two sphere boundary component. Furthermore, $T_{p}$ passes through the point $p$. For $\mathbb{A}=\mathbb{F}[\mathfrak{u}]$, there is a natural $\mathbb{A}$-coloring $\mathfrak{u}_{p}$ of $T_{p}$ which labels $T_{p}$ by $\mathfrak{u}$. Then, for any $\operatorname{Spin}^{c}$ structure $\mathfrak{s} \in \operatorname{Spin}^{c}(Y)$, setting the $\mathbb{A}$ module $\mathbb{M}$ equal to $\mathbb{F}, \mathbb{F}[\mathfrak{u}], \mathbb{F}\left[\mathfrak{u}, \mathfrak{u}^{-1}\right]$ and $\mathbb{F}\left[\mathfrak{u}^{-1}\right]$ the tangle Floer homology $\operatorname{HF}^{\mathbb{M}}\left(Y_{p}, T_{p}, \mathfrak{s}, \mathfrak{u}\right)$ is equal to $\widehat{\mathrm{HF}}(Y, \mathfrak{s}), \operatorname{HF}^{-}(Y, \mathfrak{s}), \operatorname{HF}^{\infty}(Y, \mathfrak{s})$ and $\operatorname{HF}^{+}(Y, \mathfrak{s})$, respectively. 
(c) Suppose that $L=\amalg_{i=1}^{m} L_{i}$ is an oriented link in a connected, oriented, closed 3-manifold $Y$ and $\mathbf{p}=\left\{p_{1}, \ldots, p_{n}\right\}$ is a collection of markings on $L$, so that each component of $L$ contains at least one marked point. We may consider a collection of $n$ small arcs on $L$ containing $\mathbf{p}$, and remove small balls from a neighborhood of the endpoints of these arcs. This gives a 3 -manifold $Y_{\mathbf{p}}$ with $2 n$ sphere boundary components. The orientation on $L$ may be used to decompose this boundary into $n$ spheres in $\partial^{+} Y_{\mathbf{p}}$ and $n$ spheres in $\partial^{-} Y_{\mathbf{p}}$. After changing the orientation of the small arcs, we also obtain a tangle $L_{\mathbf{p}}$ with $2 n$ connected components which connect the negative boundary to the positive boundary. Let $\mathbb{A}=\mathbb{F}\left[\mathfrak{u}_{1}, \ldots, \mathfrak{u}_{n}, \mathfrak{v}_{1}, . ., \mathfrak{v}_{m}\right]$, and label the small arc containing $p_{i}$ by $\mathfrak{u}_{i}$ and the remaining arcs on $L_{i}$ by $\mathfrak{v}_{i}$. This gives an $\mathbb{A}$-coloring of $\left(Y_{\mathbf{p}}, L_{\mathbf{p}}\right)$, denote by $\mathfrak{u}_{\mathbf{p}}$, which assigns the variables $\mathfrak{u}_{1}, \ldots, \mathfrak{u}_{n}$ to the marked points in $\mathbf{p}$ and the variables $\mathfrak{v}_{1}, \ldots, \mathfrak{v}_{m}$ to the connected components of the link $L$. For any $\operatorname{Spin}^{c}$ structure $\mathfrak{s} \in \operatorname{Spin}^{c}(Y)$, the tangle Floer homology groups $\operatorname{HF}^{\mathbb{M}}\left(Y_{\mathbf{p}}, L_{\mathbf{p}}, \mathfrak{u}_{\mathbf{p}}, \mathfrak{s}\right)$ give the usual link Floer homology groups associated with $L$ using different $\mathbb{A}$-modules $\mathbb{M}$. In particular, for any knot $K \subset S^{3}$ with one based point $p$, $\operatorname{HF}^{\frac{\mathbb{F}[\mathfrak{u}, \mathfrak{v}]}{\langle\mathfrak{v}\rangle}}\left(S_{p}^{3}, K_{p}, \mathfrak{u}_{p}, \mathfrak{s}\right)=\operatorname{HFK}^{-}(K, \mathfrak{s})$.

1.2. Main results. Associated with a cobordism $W$ from a closed, oriented 3-manifold $M$ to another closed, oriented 3-manifold $M^{\prime}$, and a $\operatorname{Spin}^{c}$ class $\mathfrak{t} \in \operatorname{Spin}^{c}(W)$, Ozsváth and Szabó construct the homomorphisms

$$
\mathfrak{f}_{W, \mathfrak{t}}^{\circ}: \operatorname{HF}^{\circ}\left(M,\left.\mathfrak{t}\right|_{M}\right) \longrightarrow \operatorname{HF}^{\circ}\left(M^{\prime},\left.\mathfrak{t}\right|_{M^{\prime}}\right) .
$$

With Theorem 1.1 in place, it is natural to ask if the construction of Ozsváth and Szabó may be extended to an invariant for cobordisms between tangles. First, we define the notion of cobordisms between tangles, by generalizing the notion of cobordisms between classical tangles.

Definition 1.5. A cobordism $(W, F)$ from $(M, T)$ to $\left(M^{\prime}, T^{\prime}\right)$ consists of a smooth oriented fourmanifold $W$, with boundary and corners and without closed components, and a properly embedded smooth oriented surface $F$ in $W$, with boundary and corners and without closed components, such that:

(1) The boundary $(\partial W, \partial F)$ of $(W, F)$ consists of a horizontal part

$$
\begin{aligned}
\left(\partial_{h} W, \partial_{h} F\right) & =\left(\partial_{h}^{+} W, \partial_{h}^{+} F\right) \amalg\left(\partial_{h}^{-} W, \partial_{h}^{-} F\right) \\
& =\left(\partial^{+} M \times I, \partial^{+} T \times I\right) \amalg\left(\partial^{-} M \times I, \partial^{-} T \times I\right) \\
& =\left(\partial^{+} M^{\prime} \times I, \partial^{+} T^{\prime} \times I\right) \amalg\left(\partial^{-} M^{\prime} \times I, \partial^{-} T^{\prime} \times I\right)
\end{aligned}
$$

and a vertical part $\left(\partial_{v} W, \partial_{v} F\right)=-(M, T) \amalg\left(M^{\prime}, T^{\prime}\right)$, with corners

$$
\left(\partial_{v} W, \partial_{v} F\right) \cap\left(\partial_{h} W, \partial_{h} F\right)=(\partial M, \partial T) \amalg\left(\partial M^{\prime}, \partial T^{\prime}\right) .
$$

(2) For every component $F_{\circ}$ of $F$ the orientation induced on $\partial F_{\circ}$ by the orientation of $F_{\circ}$ agrees with the orientation inherited from $-T \amalg T^{\prime}$.

The cobordism $(W, F)$ is called stable if $(M, T)$ and $\left(M^{\prime}, T^{\prime}\right)$ are balanced and for every connected component $F_{\circ}$ of $F$ which is not homeomorphic to a disk, $T \cap F_{\circ}$ and $T \cap F_{\circ}$ have more than one connected component.

Assume that $(W, F)$ is a stable cobordism from a balanced tangle $(M, T)$ to a balanced tangle $\left(M^{\prime}, T^{\prime}\right)$, as above. The inclusions of $T$ and $T^{\prime}$ in $F$ induce maps

$$
\jmath_{T}: \pi_{0}(T) \rightarrow \pi_{0}(F) \quad \text { and } \quad \jmath_{T^{\prime}}: \pi_{0}\left(T^{\prime}\right) \rightarrow \pi_{0}(F) .
$$

Definition 1.6. An $\mathbb{A}$-coloring for $(W, F)$ is a map $\mathfrak{u}: \pi_{0}(F) \rightarrow \mathbb{A}$ such that $\mathfrak{u} \circ \jmath_{T}$ and $\mathfrak{u} \circ \jmath_{T^{\prime}}$ are $\mathbb{A}$-colorings for $(M, T)$ and $\left(M^{\prime}, T^{\prime}\right)$, respectively.

Let $\operatorname{Spin}^{c}(W)$ denote the set of $\operatorname{Spin}^{c}$ structures on $W$ (Definition 2.4). By an affine set of $\operatorname{Spin}^{c}$ structures over $W$ we mean a subset $\mathfrak{T}=\mathfrak{t}+H_{\mathfrak{T}} \subset \operatorname{Spin}^{c}(W)$ which is determined by a $\operatorname{Spin}^{c}$ 
structure $\mathfrak{t} \in \operatorname{Spin}^{c}(W)$ and a submodule of $\operatorname{Ker}(\pi) \subset H^{2}(W, \mathbb{Z})$. Here, $\pi$ is the map from the cohomology long exact sequence

$$
\cdots \longrightarrow H^{2}\left(W, M \amalg M^{\prime}, \mathbb{Z}\right) \stackrel{\imath}{\longrightarrow} H^{2}(W, \mathbb{Z}) \stackrel{\pi}{\longrightarrow} H^{2}\left(M \amalg M^{\prime}, \mathbb{Z}\right) \longrightarrow \cdots
$$

for $\left(W, M \amalg M^{\prime}\right)$. In particular, if this fixed submodule is trivial, then $\mathfrak{T}$ consists of a single $\operatorname{Spin}^{c}$ structure $\mathfrak{t}$. If $\mathfrak{t}-\mathfrak{t}^{\prime} \in \operatorname{ker}(\pi)$, then $\left.\mathfrak{t}\right|_{M}=\left.\mathfrak{t}^{\prime}\right|_{M}$ and $\left.\mathfrak{t}\right|_{M^{\prime}}=\left.\mathfrak{t}^{\prime}\right|_{M^{\prime}}$, so $\left.\mathfrak{T}\right|_{M}$ and $\left.\mathfrak{T}\right|_{M^{\prime}}$ are well-defined.

Definition 1.7. An $\mathbb{A}$-cobordism $\mathcal{C}=[W, F, \mathfrak{T}, \mathfrak{u}]$ from $\mathcal{T}$ to $\mathcal{T}^{\prime}$ consists of a stable cobordism $(W, F)$ from $(M, T)$ to $\left(M^{\prime}, T^{\prime}\right)$, an affine set $\mathfrak{T} \subset \operatorname{Spin}^{c}(W)$ of $\operatorname{Spin}^{c}$ structure over $W$, and an $\mathbb{A}$-coloring $\mathfrak{u}: \pi_{0}(F) \rightarrow \mathbb{A}$ so that

$$
\mathcal{T}=\left[M, T, \mathfrak{s}=\left.\mathfrak{T}\right|_{M}, \mathfrak{u} \circ \jmath_{T}\right] \quad \text { and } \quad \mathcal{T}^{\prime}=\left[M^{\prime}, T^{\prime}, \mathfrak{s}^{\prime}=\left.\mathfrak{T}\right|_{M^{\prime}}, \mathfrak{u} \circ \jmath_{T^{\prime}}\right] .
$$

If $\mathcal{C}$ is an $\mathbb{A}$-cobordism from $\mathcal{T}$ to $\mathcal{T}^{\prime}$, we write $\mathcal{C}: \mathcal{T} \leadsto \mathcal{T}^{\prime}$.

The 4-tuple associated with an $\mathbb{A}$-cobordism $\mathcal{C}$ is denoted by $\left[W_{\mathcal{C}}, F_{\mathcal{C}}, \mathfrak{T}_{\mathcal{C}}, \mathfrak{u}_{\mathcal{C}}\right]$ and we set $\operatorname{Spin}^{c}(\mathcal{C})=$ $\operatorname{Spin}^{c}\left(W_{\mathcal{C}}\right)$. When $\mathfrak{T}$ consists of a single $\operatorname{Spin}^{c}$ structure $\mathfrak{t}$, we abuse the notation and denote it by $\mathfrak{t}$ (or use similar notation for it).

Using affine sets of Spin ${ }^{c}$ structures instead of single $\operatorname{Spin}^{c}$ structures over cobordisms allows us to compose $\mathbb{A}$-cobordisms. In fact, if

$$
\mathcal{C}_{1}=\left[W_{1}, F_{1}, \mathfrak{T}_{1}, \mathfrak{u}_{1}\right]: \mathcal{T} \leadsto \mathcal{T}^{\prime} \quad \text { and } \quad \mathcal{C}_{2}=\left[W_{2}, F_{2}, \mathfrak{T}_{2}, \mathfrak{u}_{2}\right]: \mathcal{T}^{\prime} \leadsto \mathcal{T}^{\prime \prime}
$$

are $\mathbb{A}$-cobordisms, then the Mayer-Vietoris exact sequence

$$
\cdots \longrightarrow H^{1}\left(M_{\mathcal{T}^{\prime}}, \mathbb{Z}\right) \stackrel{\delta}{\longrightarrow} H^{2}(W, \mathbb{Z}) \stackrel{\pi}{\longrightarrow} H^{2}\left(W_{1}, \mathbb{Z}\right) \oplus H^{2}\left(W_{2}, \mathbb{Z}\right) \longrightarrow \cdots
$$

for $W=W_{1} \cup W_{2}$, and the submodules corresponding to $\mathfrak{T}_{1}$ and $\mathfrak{T}_{2}$ determine a submodule of $H^{2}(W, \mathbb{Z})$ as their pre-image under $\pi$. Since $\mathfrak{T}_{1}$ restricts to $\mathfrak{s}=\mathfrak{s}_{\mathcal{T}}$ on $M=M_{\mathcal{T}}$ and $\mathfrak{T}_{2}$ restricts to $\mathfrak{s}^{\prime \prime}=\mathfrak{s}_{\mathcal{T}^{\prime \prime}}$ on $M^{\prime \prime}=M_{\mathcal{T}^{\prime \prime}}$, this submodule determines an affine set $\mathfrak{T}$ of $\operatorname{Spin}^{c}$ structures on $W$, which is bigger than $\mathfrak{T}_{1} \times \mathfrak{T}_{2}$ unless the map $\delta$ in the above sequence is trivial. We may then compose the $\mathbb{A}$-cobordisms $\mathcal{C}_{1}$ and $\mathcal{C}_{2}$ to obtain

$$
\mathcal{C}=\mathcal{C}_{1} \cup \cup_{\mathcal{T}^{\prime}} \mathcal{C}_{2}: \mathcal{T} \leadsto \mathcal{T}^{\prime \prime}
$$

Definition 1.7 gives a category $\mathbb{A}$-Tangles. The objects of $\mathbb{A}$-Tangles are $\mathbb{A}$-tangles and the morphisms are $\mathbb{A}$-cobordisms. The category $\mathbb{A}$-Tang is a subcategory of $\mathbb{A}$-Tangles: given a diffeomorphism

$$
d:[M, T, \mathfrak{s}, \mathfrak{u}] \rightarrow\left[M^{\prime}, T^{\prime}, \mathfrak{s}^{\prime}, \mathfrak{u}^{\prime}\right]
$$

define an $\mathbb{A}$-cobordism $\mathcal{C}=\left[W, F, \mathfrak{t}, \mathfrak{u}_{F}\right]$, where $(W, F)=(M, T) \times[0,1]$, and $(M, T) \times\{1\}$ is identified with $\left(M^{\prime}, T^{\prime}\right)$ by the diffeomorphism $d$. Further, the Spin ${ }^{c}$ structure $\mathfrak{t}$ and the map $\mathfrak{u}_{F}$ are trivially determined by $\mathfrak{s}$ and $\mathfrak{u}$, respectively.

For every $\mathbb{A}$-cobordism $\mathcal{C}$ from an $\mathbb{A}$-tangle $\mathcal{T}$ to an $\mathbb{A}$-tangle $\mathcal{T}^{\prime}$, and any $\mathbb{A}$-module $\mathbb{M}$, we define an $\mathbb{A}$-homomorphism

$$
\mathfrak{f}_{\mathcal{C}}^{\mathbb{M}}: \operatorname{HF}^{\mathbb{M}}(\mathcal{T}) \rightarrow \operatorname{HF}\left(\mathcal{T}^{\prime}\right)
$$

Theorem 1.2. For every $\mathbb{F}$-algebra $\mathbb{A}$, the functor $\mathrm{HF}^{\mathbb{M}}$ from Theorem 1.1, extends to a functor

$$
\mathrm{HF}^{\mathbb{M}}: \mathbb{A} \text {-Tangles } \longrightarrow \mathbb{A} \text {-Modules }
$$

by setting $\operatorname{HF}^{\mathbb{M}}(\mathcal{C})=\mathfrak{f}_{\mathcal{C}}^{\mathbb{M}}$, for any $\mathbb{A}$-cobordism $\mathcal{C}$. Here, $\mathbb{A}$-Modules denotes the category of $\mathbb{A}$ modules with $\mathbb{A}$-homomorphisms between them.

If $\phi: \mathbb{M} \rightarrow \mathbb{M}^{\prime}$ is a homomorphism of $\mathbb{A}$-modules, we obtain a corresponding homomorphism of A-modules

$$
\mathfrak{f}^{\phi}: \operatorname{HF}^{\mathbb{M}}(\mathcal{T}) \longrightarrow \operatorname{HF}^{\mathbb{M}^{\prime}}(\mathcal{T})
$$


Given an $\mathbb{A}$-cobordism $\mathcal{C}: \mathcal{T} \leadsto \mathcal{T}^{\prime}$, the following diagram is commutative:

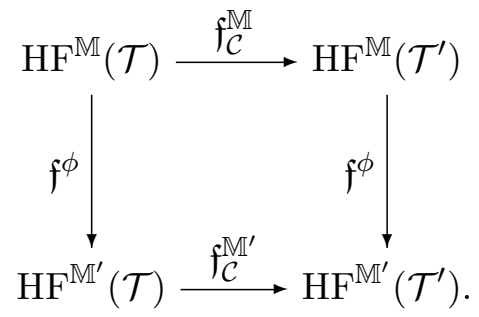

Moreover, given a short exact sequence

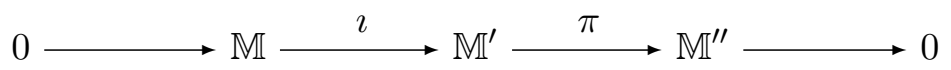

of $\mathbb{A}$-modules, we obtain a corresponding exact triangle of tangle Floer homology $\mathbb{A}$-modules:

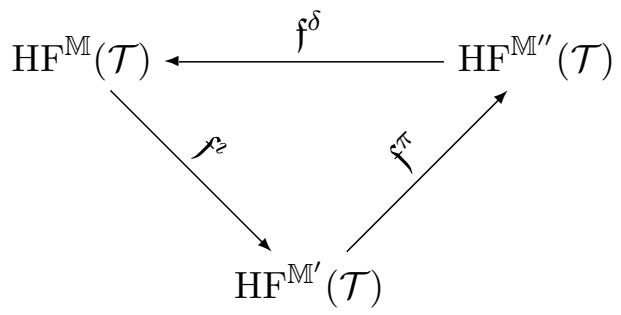

where $\mathfrak{f}^{\delta}$ is a connecting homomorphism.

1.3. Examples and applications. Let us first review some of the familiar cases of the above construction.

Example 1.2. Let $\mathcal{Y}=(Y, p)$ and $\mathcal{Y}^{\prime}=\left(Y^{\prime}, p^{\prime}\right)$ be pointed, oriented, connected and closed 3manifolds. If $X$ is a smooth 4-dimensional cobordism from $Y$ to $Y^{\prime}$ and if $\sigma$ is a simple path in $X$ from $p$ to $p^{\prime}$, then $\mathcal{X}=(X, \sigma)$ gives a cobordism $\left(X_{\sigma}, F_{\sigma}\right)$ from $\left(Y_{p}, T_{p}\right)$ to $\left(Y_{p^{\prime}}, T_{p^{\prime}}\right)$, where $F_{\sigma}$ is a disk. Let $\mathfrak{u}_{\sigma}$ be the $\mathbb{F}\left[\mathfrak{u}\right.$-coloring that labels $F_{\sigma}$ by $\mathfrak{u}$. For every $\operatorname{Spin}^{c} \operatorname{structure}^{t} \in \operatorname{Spin}^{c}(X)$, $\mathcal{C}_{\mathcal{X}, \mathfrak{t}}=\left(X_{\sigma}, F_{\sigma}, \mathfrak{t}, \mathfrak{u}_{\sigma}\right)$ is an $\mathbb{A}$-cobordism from $\mathcal{T}_{\mathcal{Y}, \mathfrak{s}}$ to $\mathcal{T}_{\mathcal{Y}^{\prime}, \mathfrak{s}^{\prime}}$, where $\mathfrak{s}=\left.\mathfrak{t}\right|_{Y_{p}}$ and $\mathfrak{s}^{\prime}=\left.\mathfrak{t}\right|_{Y_{p^{\prime}}^{\prime}}$. In view of Example 1.1 (part (a)), when we choose $\mathbb{M}$ equal to $\mathbb{F}, \mathbb{F}[\mathfrak{u}], \mathbb{F}\left[\mathfrak{u}^{-1}\right]$ or $\mathbb{F}\left[\mathfrak{u}, \mathfrak{u}^{-1}\right]$, the $\mathbb{A}$-homomorphism $\operatorname{HF}^{\mathbb{M}}\left(\mathcal{C}_{\mathcal{X}, t}\right)=\mathfrak{f}_{\mathcal{C}_{\mathcal{X}, t}}^{\mathbb{M}}$ is the cobordism map of Ozsváth and Szabó in the corresponding cases.

Example 1.3. (Functoriality of knot and link Floer homologies) Suppose that $(L, \mathbf{p}) \subset Y$ and $\left(L^{\prime}, \mathbf{p}^{\prime}\right) \subset Y^{\prime}$ are marked links, as in Example 1.1, with $|\mathbf{p}|=\left|\mathbf{p}^{\prime}\right|=n$. For a decorated cobordism $\mathcal{Z}=(Z, F, \sigma)$ consisting of a smooth, oriented, 4-dimensional cobordism $Z$ from $Y$ to $Y^{\prime}$, a properly embedded, smooth, oriented surface $F \subset Z$ which connects $L$ to $L^{\prime}$ and a properly embedded, oriented 1-manifold $\sigma \subset F$ which connects $\mathbf{p}$ to $\mathbf{p}^{\prime}$ i.e. $\partial^{-} \sigma=\mathbf{p}$ and $\partial^{+} \sigma=\mathbf{p}^{\prime}$, we construct a cobordism $\left(X_{\sigma}, F_{\sigma}\right)$ connecting $\left(Y_{\mathbf{p}}, L_{\mathbf{p}}\right)$ to $\left(Y_{\mathbf{p}^{\prime}}^{\prime}, L_{\mathbf{p}^{\prime}}^{\prime}\right)$. We require that $\sigma$ does not have any closed components and that any connected component of $F-\sigma$ with positive genus intersects $L$ and $L^{\prime}$ in more than one connected component, to achieve stability. Then the connected components $\sigma_{1}, \ldots, \sigma_{n}$ give a matching between $\mathbf{p}$ and $\mathbf{p}^{\prime}$. We may thus assume that the endpints of $\sigma_{i}$ are $p_{i} \in \mathbf{p}$ and $p_{i}^{\prime} \in \mathbf{p}^{\prime}$, for $i=1, \ldots, n$.

Let us assume that $F=\coprod_{j=1}^{m} F_{j}$ and set $\mathbb{A}=\mathbb{F}\left[\mathfrak{u}_{1}, \ldots, \mathfrak{u}_{n}, \mathfrak{v}_{1}, \ldots, \mathfrak{v}_{m}\right]$. The $\mathbb{A}$-coloring of $\left(X_{\sigma}, F_{\sigma}\right)$ is given by labeling the disk associated with each $\sigma_{i}$ by $\mathfrak{u}_{i}$, and labeling each connected component of $F_{j} \backslash \sigma$ by $\mathfrak{v}_{j}$. If $\mathbb{A}_{L, \mathbf{p}}$ denotes the ring associated with the marked link $(L, \mathbf{p})$, there is a quotient map from $\mathbb{A}_{L, \mathbf{p}}$ to $\mathbb{A}$. If two link components in $L$ are on the boundary of the same connected component of $F$, the variables associated to these link components by $\mathfrak{u}_{\mathbf{p}}$ are identified in the quotient. In particular, if each connected component of $F$ intersects precisely one connected component of each one $L$ and $L^{\prime}, \mathbb{A}_{L, \mathbf{p}}$ and $\mathbb{A}_{L^{\prime}, \mathbf{p}^{\prime}}$ are both identified with $\mathbb{A}$. In this case, we call $(Z, F, \sigma)$ a decorated link cobordism from $(Y, L, \mathbf{p})$ to $\left(Y^{\prime}, L^{\prime}, \mathbf{p}^{\prime}\right)$. 
Let us assume that $(Z, F, \sigma)$ is a decorated link cobordism from $(Y, L, \mathbf{p})$ to $\left(Y^{\prime}, L^{\prime}, \mathbf{p}^{\prime}\right)$. Associated with every $\operatorname{Spin}^{c}$ structure $\mathfrak{t} \in \operatorname{Spin}^{c}(Z)$ we obtain an $\mathbb{A}$-cobordism

$$
\mathcal{C}_{\mathcal{Z}, \mathfrak{t}}=\left[W_{\mathcal{Z}}, F_{\mathcal{Z}}, \mathfrak{t}, \mathfrak{u}_{\mathcal{Z}}\right]: \mathcal{T}_{L, \mathbf{p},\left.\mathfrak{t}\right|_{Y}} \leadsto \mathcal{T}_{L^{\prime}, \mathbf{p}^{\prime},\left.\mathfrak{t}\right|_{Y^{\prime}}}
$$

Correspondingly, we obtain the cobordism maps

$$
\mathfrak{f}_{\mathcal{Z}, \mathfrak{t}}^{\mathbb{M}}: \operatorname{HF}^{\mathbb{M}}\left(Y, L, \mathbf{p},\left.\mathfrak{t}\right|_{Y}\right) \rightarrow \operatorname{HF}^{\mathbb{M}}\left(L^{\prime}, \mathbf{p}^{\prime},\left.\mathfrak{t}\right|_{Y^{\prime}}\right) .
$$

The functoriality of link Floer homology then follows from our main theorem.

Remark 1.3. A similar construction, in the context of pointed links and cobordisms between them, is independently given by Ian Zemke in [Zem].

Suppose that the oriented knots $K$ and $K^{\prime}$ differ by changing one crossing. Corresponding to this crossing change there is a cobordism obtained by a band attachment from $K$ to $L=K^{\prime} \# H$, where $H$ is the right- or left-handed Hopf link. In AE, we use the corresponding cobordism maps for appropriate choices of based points on $K$ and $L$ and decoration on the cobordism, and we define a lower bound $\mathfrak{l}(K)$ on the unknotting number of $K$. This bound is greater than or equal to $\nu^{-}(K), \nu^{-}(-K)$ and the order of $U$-torsions in $\operatorname{HFK}^{-}(K)$. Additionally, it only vanishes for the unknot, and we present examples of slice knots $K$ such that $\mathfrak{l}(K)$ is arbitrarily large. A parallel construction is used by the first author in [Ali] to construct lower bounds on the unknotting number from Khovanov homology, also see [AD].

1.4. Outline of the paper. The paper is organized as follows. In Section 2 we review Heegaard poly-tuples and $\mathrm{Spin}^{c}$ structures over them. Then in Section 3 we follow the footsteps of Juhász and Thurston [JT] to show that tangle Floer homology for $\mathbb{A}$-tangles gives functors from $\mathbb{A}$-Tang to A-Mod. In Section 4 we study parametrized Cerf decompositions of cobordisms between tangles. We show that any two parametrized Cerf decompositions for a stable cobordism can be connected by a sequence of Cerf moves.

In Section 5 we define cobordism maps for parametrized cobordisms associated with attaching one or three handles and show that the map is invariant. In Section 6 we introduce a special $\mathbb{A}$-tangle $\mathcal{T}_{F}$ associated to the positive boundary of any $\mathbb{A}$-cobordism together with a distinguished generator $\Theta_{F} \in \operatorname{HF}\left(\mathcal{T}_{F}\right)$. The distinguished generator makes it possible to define invariant cobordism maps for cobordisms parametrized with framed links and framed arcs. In Section 7 we define cobordism maps for arbitrary $\mathbb{A}$-cobordisms by composing cobordism maps constructed in Sections 5 and 6 for cobordisms parametrized by framed 0 -spheres, framed knots and arcs and framed 2 -spheres. We prove that this map is in fact an invariant and does not depend on the parametrized Cerf decomposition. Moreover, we show that this construction gives functors from $\mathbb{A}$-Tangles to A-Modules. Finally in Section 8 we discuss some special cases and applications. In particular, decorated cobordisms between pointed links induce functorial maps on link Floer homology.

Acknowledgements. The authors would like to thank András Juhász and Robert Lipshitz for helpful discussions and suggestions. Most of this work was done when the first author was a postdoc at Max Planck Institute for Mathematics (MPIM) in Bonn. She gratefully acknowledges the support and the hospitality of MPIM through this period. 


\section{Tangles, Spin $^{c}$ Structures and HeegaArd poly-tuples}

In this section, we describe the correspondence between sutured manifolds and tangles. Then, we review some definitions and results from [AE15] about Heegaard Floer homology for tangles (sutured manifolds), to fix our notation. We will also reformulate some definitions and results about Heegaard diagrams, Heegaard poly-tuples and $\mathrm{Spin}^{c}$ structures from [AE15, GW10, OS04c, OS06] to work in our setup.

2.1. Sutured manifolds and tangles. Sutured manifolds were introduced by Gabai in Gab83, Gab87a, Gab87b]. Throughout this paper, we use a less general family of sutured manifolds by excluding toroidal sutures.

Definition 2.1. A sutured manifold $(X, \tau)$ is an oriented 3-manifold $X$ with boundary, together with a set of pairwise disjoint, oriented, simple closed curves $\tau=\left\{\tau_{1}, \ldots, \tau_{\kappa}\right\}$ on $\partial X$. We will denote by $A\left(\tau_{i}\right)$ a tubular neighborhood of $\tau_{i}$ in $\partial X$, which is an annulus. We let $A(\tau)=A\left(\tau_{1}\right) \amalg \cdots \amalg A\left(\tau_{\kappa}\right)$. Every connected component of $\mathfrak{R}(\tau)=\partial X \backslash A(\tau)^{\circ}$ is oriented with the orientation induced from $X$, where $A(\tau)^{\circ}$ denotes the interior of $A(\tau)$. Furthermore, we require that $\mathfrak{R}(\tau)=\mathfrak{R}^{+}(\tau) \amalg$ $\mathfrak{R}^{-}(\tau)$ where $\mathfrak{R}^{+}(\tau)$ (respectively, $\mathfrak{R}^{-}(\tau)$ ) denotes the union of components of $\mathfrak{R}(\tau)$ such that the orientation induced on $\tau$ as the boundary of $\mathfrak{R}^{+}(\tau)$ (respectively, $\mathfrak{R}^{-}(\tau)$ ) agrees with (respectively, is the opposite of) the orientation of $\tau$.

Every sutured manifold $(X, \tau)$ determines a tangle $(M, T)$, where $M=\bar{X}$ is obtained from $X$ by filling the sutures (i.e. attaching 2-handles along the sutures) and $T$ is the set of cocores of these 2-handles. The orientation on $\tau$ induces an orientation on $T$ and the decomposition $\partial \mathfrak{R}(\tau)=\mathfrak{R}^{+}(\tau) \amalg \mathfrak{R}^{-}(\tau)$ induces a decomposition $\partial M=\partial^{+} M \amalg \partial^{-} M$ of the boundary of $M$. Conversely, every tangle $(M, T)$ determines a sutured manifold $(X, \tau)$ where $X=M \backslash \operatorname{nd}(T)$ and $\tau$ is the set of meridians of $T$ along with the induced orientation.

Note that a tangle $(M, T)$ is balanced if and only if the corresponding sutured manifold is balanced in the sense of [Juh06, Definition 2.2].

In AE15, we introduced a $\mathbb{Z}$-algebra $\mathbb{A}_{\tau}$ associated to the boundary of any balanced sutured manifold $(X, \tau)$. Assume $\tau=\amalg_{i=1}^{\kappa} \tau_{i}$,

$$
\mathfrak{R}^{-}(\tau)=\coprod_{i=1}^{k} R_{i}^{-} \quad \text { and } \quad \mathfrak{R}^{+}(\tau)=\coprod_{j=1}^{l} R_{j}^{+} .
$$

Associated with the connected components of $\mathfrak{R}(\tau)$, consider the elements

$$
\mathfrak{u}_{i}^{-}:=\prod_{\tau_{j} \subset \partial R_{i}^{-}} \mathfrak{u}_{j}, \quad i=1, \ldots, k \quad \text { and } \quad \mathfrak{u}_{i}^{+}:=\prod_{\tau_{j} \subset \partial R_{i}^{+}} \mathfrak{u}_{j}, \quad i=1, \ldots, l,
$$

in the free $\mathbb{Z}$-algebra $\mathbb{Z}\left[\mathfrak{u}_{1}, \ldots, \mathfrak{u}_{\kappa}\right]$ generated by $\mathfrak{u}_{1}, \ldots, \mathfrak{u}_{\kappa}$. Then

$$
\mathbb{A}_{\tau}=\frac{\mathbb{Z}\left[\mathfrak{u}_{1}, \ldots, \mathfrak{u}_{\kappa}\right]}{\left\langle\mathfrak{u}^{+}(\tau)-\mathfrak{u}^{-}(\tau)\right\rangle+\left\langle\mathfrak{u}_{i}^{+} \mid g_{i}^{+}>0\right\rangle+\left\langle\mathfrak{u}_{i}^{-} \mid g_{i}^{-}>0\right\rangle}
$$

where $\mathfrak{u}^{-}(\tau)=\sum_{i=1}^{k} \mathfrak{u}_{i}^{-}, \mathfrak{u}^{+}(\tau)=\sum_{i=1}^{l} \mathfrak{u}_{i}^{+}$and $g_{i}^{\bullet}$ denotes the genus of $R_{i}^{\bullet}$ for $\bullet=+,-$. For any balanced tangle $(M, T)$, denote the algebra associated to its corresponding balanced sutured manifold by $\mathbb{A}_{T}$.

Suppose $(M, T)$ be a connected balanced tangle (i.e. a balanced tangle with $M$ connected). The map $\mathfrak{u}_{T}: \pi_{0}(T) \rightarrow \mathbb{A}_{T}$ which sends each component $T_{i}$ to the variable $\mathfrak{u}_{i}$ corresponding to its meridian gives an $\mathbb{A}_{T}$-coloring for $(M, T)$. In fact, any map $\mathfrak{u}: \pi_{0}(T) \rightarrow \mathbb{A}$ is an $\mathbb{A}$-coloring for $(M, T)$ if and only if $\mathfrak{u}=\phi \circ \mathfrak{u}_{T}$ for a homomorphism $\phi$ from $\mathbb{A}_{T}$ to $\mathbb{A}$.

2.2. Heegaard Floer homology for tangles. Recall that a balanced Heegaard diagram is a 4-tuple $H=(\Sigma, \boldsymbol{\alpha}, \boldsymbol{\beta}, \mathbf{z})$ where $\Sigma$ is a closed oriented surface, $\boldsymbol{\alpha}$ and $\boldsymbol{\beta}$ are sets of pairwise disjoint circles on $\Sigma$, and $\mathbf{z} \subset \Sigma \backslash(\boldsymbol{\alpha} \cup \boldsymbol{\beta})$ is a set of points. Further, $|\boldsymbol{\alpha}|=|\boldsymbol{\beta}|$ and $\mathbf{z}$ intersects every connected component of $\Sigma \backslash \boldsymbol{\alpha}$ and $\Sigma \backslash \boldsymbol{\beta}$. Every balanced Heegaard diagram $H$, specifies a balanced tangle 
$(M, T)$, where $M$ is obtained from $\Sigma \times[0,1]$ by attaching 2-handles along the circles $\boldsymbol{\alpha} \times\{0\}$ and $\boldsymbol{\beta} \times\{1\}$, and $T=\mathbf{z} \times[0,1]$.

A Heegaard diagram for a balanced tangle $(M, T)$, is a balanced Heegaard diagram $H=$ $(\Sigma, \boldsymbol{\alpha}, \boldsymbol{\beta}, \mathbf{z})$, so that $\Sigma$ is an embedded separating surface in $M$ which cuts $T$ transversely in $\mathbf{z}$, $\boldsymbol{\alpha}$ and $\boldsymbol{\beta}$ bound disjoint disks on the two sides of $\Sigma$. Moreover, let $\Sigma[\boldsymbol{\alpha}]$ and $\Sigma[\boldsymbol{\beta}]$ be embedded surfaces obtained from compressing $\Sigma$ along the $\boldsymbol{\alpha}$ and $\boldsymbol{\beta}$ curves, respectively. Then, $(\Sigma[\boldsymbol{\alpha}], \mathbf{z})$ and $(\Sigma[\boldsymbol{\beta}], \mathbf{z})$ are isotopic relative to $T$ to $\left(\partial^{-} M, \partial^{-} T\right)$ and $\left(\partial^{+} M, \partial^{+} T\right)$, respectively.

Let $H=\left(\Sigma, \boldsymbol{\alpha}, \boldsymbol{\beta}, \mathbf{z}=\left\{z_{1}, \ldots, z_{\kappa}\right\}\right)$ be a Heegaard diagram which corresponds to a balanced tangle $(M, T)$. We set $\operatorname{Spin}^{c}(H)$ equal to $\operatorname{Spin}^{c}(M)$. For an $\mathbb{F}$-algebra $\mathbb{A}$, consider a map $\mathfrak{u}: \mathbf{z} \rightarrow \mathbb{A}$. Then, corresponding to any 2-chain $\mathcal{D}$ on $\Sigma$ with boundary on $\boldsymbol{\alpha} \cup \boldsymbol{\beta}$, let $n_{i}(\mathcal{D})$ denote the coefficient of $\mathcal{D}$ at $z_{i}$ and set

$$
\mathfrak{u}(\mathcal{D}):=\prod_{i=1}^{\kappa} \mathfrak{u}_{i}^{n_{i}(\mathcal{D})} \in \mathbb{A},
$$

where $\mathfrak{u}_{i}=\mathfrak{u}\left(z_{i}\right)$. Let $\Sigma \backslash \boldsymbol{\alpha}=\amalg_{i=1}^{k} A_{i}$ and $\Sigma \backslash \boldsymbol{\beta}=\amalg_{j=1}^{l} B_{j}$.

Definition 2.2. With the above notation fixed, $\mathfrak{u}$ is called an $\mathbb{A}$-coloring for $H$ if it satisfies the following two conditions.

- $\sum_{i=1}^{k} \mathfrak{u}\left(A_{i}\right)=\sum_{j=1}^{l} \mathfrak{u}\left(B_{j}\right)$

- if $A_{i}$ or $B_{j}$ is not a punctured sphere, then $\mathfrak{u}\left(A_{i}\right)=0$ or $\mathfrak{u}\left(B_{j}\right)=0$, respectively.

Definition 2.3. A Heegaard diagram $H$, together with an $\mathbb{A}$-coloring $\mathfrak{u}$ and a $\operatorname{Spin}^{c}$ class $\mathfrak{s} \in$ $\operatorname{Spin}^{c}(H)$ is called an $\mathbb{A}$-diagram, if $(H, \mathfrak{u})$ is $\mathfrak{s}$-admissible. Here, $(H, \mathfrak{u})$ is called $\mathfrak{s}$-admissible if for any periodic domain $\mathcal{P}$ with $\left\langle c_{1}(\mathfrak{s}), H(\mathcal{P})\right\rangle=0$, either $\mathfrak{u}(\mathcal{P})=0$ or the coefficient of $\mathcal{P}$ at some point is negative.

Any $\mathbb{A}$-diagram specifies an $\mathbb{A}$-tangle. Given an $\mathbb{A}$-tangle $\mathcal{T}=[M, T, \mathfrak{s}, \mathfrak{u}]$, an $\mathbb{A}$-diagram for $\mathcal{T}$ consists of a Heegaard diagram for $(M, T)$, together with the $\mathbb{A}$-coloring induced by $\mathfrak{u}$ and the $\operatorname{Spin}^{c}$ class induced by $\mathfrak{s}$.

Let $\mathcal{H}=(\Sigma, \boldsymbol{\alpha}, \boldsymbol{\beta}, \mathfrak{u}: \mathbf{z} \rightarrow \mathbb{A}, \mathfrak{s})$ be an $\mathbb{A}$-diagram for the $\mathbb{A}$-tangle $\mathcal{T}$. Choose a generic path $J_{s}$ of almost complex structures on $\operatorname{Sym}^{\ell}(\Sigma)$, where $\ell=|\boldsymbol{\alpha}|=|\boldsymbol{\beta}|$. In [AE15, we construct a chain complex

$$
\mathrm{CF}_{J_{s}}(\Sigma, \boldsymbol{\alpha}, \boldsymbol{\beta}, \mathfrak{u}, \mathfrak{s})
$$

which is generated by the intersection points $\mathbf{x} \in \mathbb{T}_{\alpha} \cap \mathbb{T}_{\beta}$ with $\mathfrak{s}(\mathbf{x})=\mathfrak{s}$, and its chain homotopy type is an invariant of $\mathcal{T}$. We usually drop $J_{s}$ from the notation for simplicity. Furthermore, for any $\mathbb{A}$-module $\mathbb{M}$ the isomorphism type of the Floer homology group

$$
\operatorname{HF}^{\mathbb{M}}(\mathcal{T}):=H_{\star}\left(\mathrm{CF}(\Sigma, \boldsymbol{\alpha}, \boldsymbol{\beta}, \mathfrak{u}, \mathfrak{s}) \otimes_{\mathbb{A}} \mathbb{M}\right)
$$

is an invariant of $\mathcal{T}$. For simplicity, we set $\operatorname{HF}(\mathcal{T}):=\operatorname{HF}^{\mathbb{A}}(\mathcal{T})$. Moreover, for any balanced tangle $(M, T)$, let

$$
\operatorname{HF}(M, T, \mathfrak{s}):=\operatorname{HF}\left(M, T, \mathfrak{u}_{T}: \pi_{0}(T) \rightarrow \mathbb{A}_{T}, \mathfrak{s}\right)
$$

where $\mathbb{A}_{T}$ is the algebra associated to $(M, T)$ and $\mathfrak{u}_{T}$ is its corresponding coloring map.

2.3. $\operatorname{Spin}^{c}$ structures over cobordisms. Given a stable cobordism $(W, F)$ from $(M, T)$ to $\left(M^{\prime}, T^{\prime}\right)$, let $\xi$ denote the oriented 2-plane field in $\partial_{h} W=\partial M \times[0,1]$ consisting of the tangent planes of the surfaces $\partial M \times\{t\}$ for any $t \in[0,1]$. Fix an almost complex structure $J_{0}$ on $\left.T W\right|_{\partial_{h} W}$ such that $\xi$ consists of complex lines i.e. 2-planes in $\xi$ are invariant under $J_{0}$. One may further extend $J_{0}$ to a an almost complex structure on $F$ by requiring that $J_{0}$ preserves the tangent space of $F$. Note that the set of almost complex structures $J_{0}$ with the above property is contractible.

Definition 2.4. A $\operatorname{Spin}^{c}$ structure on $W$ is the homology class of a pair $(J, P)$, where

(1) $P \subset W \backslash \partial_{h} W$ is a finite collection of points,

(2) $J$ is an almost complex structure on $W \backslash P$ with $\left.J\right|_{\partial_{h} W}=J_{0}$. 
We call the pairs $\left(J_{1}, P_{1}\right)$ and $\left(J_{2}, P_{2}\right)$ homologous if there exists a compact 1-manifold $C \subset W \backslash \partial_{h} W$ without closed components so that $\partial C=P_{1} \cup P_{2}$, and $\left.J_{1}\right|_{W \backslash C}$ is isotopic relative to $\partial_{h} W$ to $\left.J_{2}\right|_{W \backslash C}$. Denote the set of $\operatorname{Spin}^{c}$ structures on $W$ by $\operatorname{Spin}^{c}(W)$. Similarly, a relative $\operatorname{Spin}^{c}$ structure on the pair $(W, F)$ is the homology class of a pair $(J, P)$, where $P \subset W \backslash\left(\partial_{h} W \cup F\right)$ is a finite collection of points and $J$ is an almost complex structure on $W \backslash P$ which agrees with $J_{0}$ over $\partial_{h} W \cup F$. The notion of homologous pairs is defined similarly, the only difference is that we need to consider isotopies relative $\partial_{h} W \cup F$. The set of relative $\operatorname{Spin}^{c}$ structures over $(W, F)$ is denoted by $\operatorname{Spin}^{c}(W, F)$.

Since $J_{0}$ is chosen from a contractible family, the above definition does not depend on the particular choice of $J_{0}$.

After fixing a metric over the 4-manifold $W$, any oriented 2-plane field which extends $\xi$ and is defined in the complement $W-P$ determines a corresponding almost complex structure. It is not hard to show that $\operatorname{Spin}^{c}(W)$ is an affine space over $\mathrm{H}^{2}\left(W, \partial_{h} W ; \mathbb{Z}\right)$.

For every $\operatorname{Spin}^{c}$ structure $\mathfrak{s}$ over $W$, the induced $\operatorname{Spin}^{c}$ structures $\left.\mathfrak{s}\right|_{M}$ and $\left.\mathfrak{s}\right|_{M^{\prime}}$ are defined as follows. Consider an almost complex structure $J$ (defined on $W \backslash P$ ) representing $\mathfrak{s}$. At any point $p$ in $M, J$ specifies a subspaces $V_{p}=T_{p} M \cap J\left(T_{p} M\right)$ of $T_{p} M$. Similarly, at any $p \in M^{\prime}$, it specifies a subspace $V_{p}^{\prime} \subset T_{p} M^{\prime}$. Let $V$ and $V^{\prime}$ denote the corresponding plane fields in $M$ and $M^{\prime}$, respectively. Then, $\left.\mathfrak{s}\right|_{M}$ and $\left.\mathfrak{s}\right|_{M^{\prime}}$ are defined to be the $\operatorname{Spin}^{c}$ classes represented by $V$ and $V^{\prime}$, respectively.

2.4. Heegaard poly-tuples. Let $H=\left(\Sigma, \boldsymbol{\alpha}^{1}, \ldots, \boldsymbol{\alpha}^{m}, \mathbf{z}\right)$ be a balanced Heegaard diagram. This means that $\Sigma$ is a closed oriented surface and for any $1 \leq i \leq m, \boldsymbol{\alpha}^{i}=\left\{\alpha_{1}^{i}, \ldots, \alpha_{\ell}^{i}\right\}$ is a set of $\ell$ disjoint simple closed curves on $\Sigma$ for some $\ell>0$. Moreover, $\mathbf{z}=\left\{z_{1}, \ldots, z_{\kappa}\right\}$ is a set of marked points in $\Sigma-\boldsymbol{\alpha}^{1}-\boldsymbol{\alpha}^{2}-\cdots-\boldsymbol{\alpha}^{m}$ such that for any $1 \leq i \leq m$ every connected component of $\Sigma-\boldsymbol{\alpha}^{i}$ intersects $\mathbf{z}$.

Associated with the balanced Heegaard diagram $H$, we define a pair $\left(W_{H}, F_{H}\right)$ as follows. For every $1 \leq i \leq m$, the Heegaard diagram $\left(\Sigma, \boldsymbol{\alpha}^{i}, \emptyset, \mathbf{z}\right)$ determines a tangle $\left(U_{i}, T_{i}\right)$, where $U_{i}=C\left[\boldsymbol{\alpha}^{i}\right]$ is the compression body determined by $\boldsymbol{\alpha}^{i}$ i.e. it is obtained from $\Sigma \times[0,1]$ by attaching 2 -handles along the curves $\boldsymbol{\alpha}^{i} \times\{1\}$, and $T_{i}=\mathbf{z} \times[0,1]$. Thus $\partial U_{i}=\partial^{-} U_{i} \amalg \partial^{+} U_{i}$, where

$$
\partial^{-} U_{i}=\Sigma \quad \text { and } \quad \partial^{+} U_{i}=\Sigma\left[\boldsymbol{\alpha}_{i}\right]
$$

and $\Sigma\left[\boldsymbol{\alpha}_{i}\right]$ is obtained by cutting $\Sigma$ along $\boldsymbol{\alpha}_{i}$ and attaching disks to the boundary components of the resulting surface. We denote the finite set $T_{i} \cap \partial^{-} U_{i}=\mathbf{z} \times\{0\}$ by $\mathbf{z}_{i}$.

Let $\mathbb{D}_{m}$ be a $m$-gon with the vertices $v_{1}, \ldots, v_{m}$, labelled in clockwise order, and edges $e_{1}, \ldots, e_{m}$, where $e_{i}$ connects $v_{i-1}$ to $v_{i}$ for $i=2, \ldots, m$ and $e_{1}$ connects $v_{m}$ to $v_{1}$. Define

$$
W_{H}:=\frac{\left(\Sigma \times \mathbb{D}_{m}\right) \amalg\left(\coprod_{i=1}^{m} U_{i} \times e_{i}\right)}{\Sigma \times e_{i} \sim \partial^{-} U_{i} \times e_{i}} \text { and } F_{H}:=\frac{\left(\mathbf{z} \times \mathbb{D}_{m}\right) \amalg\left(\coprod_{i=1}^{m} T_{i} \times e_{i}\right)}{\mathbf{z} \times e_{i} \sim \mathbf{z}_{i} \times e_{i}} \text {. }
$$

We smooth the corners of $W_{H}$ and $F_{H}$ along $v_{i} \times \Sigma$ for $1 \leq i \leq m$. Corresponding to any vertex $v_{i}$, we obtain a balanced tangle in $\left(\partial W_{H}, \partial F_{H}\right)$ determined by the Heegaard diagram $\left(\Sigma, \boldsymbol{\alpha}^{i}, \boldsymbol{\alpha}^{i+1}, \mathbf{z}\right)$ denoted by $\left(M_{i, i+1}, T_{i, i+1}\right)$. Note that $\boldsymbol{\alpha}^{m+1}=\boldsymbol{\alpha}^{1}$ and $\left(M_{m, m+1}, T_{m, m+1}\right)=\left(M_{m, 1}, T_{m, 1}\right)$. Let

$$
M^{\prime}:=M_{1,2} \amalg M_{2,3} \amalg \cdots \amalg M_{m, 1} \subset \partial W_{H} \quad \text { and } \quad Z:=\partial W_{H}-\operatorname{int}\left(M^{\prime}\right)
$$

Thus, $Z$ is a product and

$$
Z=\left(\partial^{-} M_{1,2} \amalg \ldots \amalg \partial^{-} M_{m, 1}\right) \times[0,1] .
$$

Fix an almost complex structure $J_{0}$ on $\left.T W_{H}\right|_{Z}$ such that for any $t \in[0,1]$ the tangent planes to the surface $\left(\partial^{-} M_{1,2} \amalg \cdots \amalg \partial^{-} M_{m, 1}\right) \times\{t\}$ are complex lines. The almost complex structure $J_{0}$ may further be extended to $F_{H}$ so that it preserves the tangent space of $F_{H}$, i.e. the tangent planes of $F_{H}$ are all complex lines. We may then talk about the Spin ${ }^{c}$ structures on $W_{H}$ and the relative Spin $^{c}$ structures on $\left(W_{H}, F_{H}\right)$. 
Definition 2.5. The set of $\operatorname{Spin}^{c}$ structures on $W_{H}$, denoted by $\operatorname{Spin}^{c}\left(W_{H}\right)$, is defined as the set of homology classes of the pairs $(J, P)$ consisting of a finite set of points $P$ in the interior of $W_{H}$ and an almost complex structure $J$ on $W_{H}-P$ such that $\left.J\right|_{Z}=J_{0}$. Similarly, the set of relative Spin ${ }^{c}$ structures on $\left(W_{H}, F_{H}\right)$, denoted by $\operatorname{Spin}^{c}\left(W_{H}, F_{H}\right)$, is defined as the set of homology classes of the pairs $(J, P)$ consisting of a finite set $P$ of points in the interior of $W_{H}-F_{H}$ and an almost complex structure $J$ on $W_{H}-P$ such that $\left.J\right|_{Z \cup F_{H}}=J_{0}$.

$\operatorname{Spin}^{c}\left(W_{H}\right)$ is an affine space over $\mathrm{H}^{2}\left(W_{H}, Z ; \mathbb{Z}\right)$, and $\operatorname{Spin}^{c}\left(W_{H}, F_{H}\right)$ is an affine space over $\mathrm{H}^{2}\left(W_{H}, Z \cup F_{H} ; \mathbb{Z}\right)$.

Let $\mathbb{T}_{i} \subset \operatorname{Sym}^{\ell}(\Sigma)$ denote the torus $\alpha_{1}^{i} \times \cdots \times \alpha_{\ell}^{i}$. Given intersection points $\mathbf{x}_{i} \in \mathbb{T}_{i} \cap \mathbb{T}_{i+1}$, the homotopy classes of maps

$$
\Psi: \mathbb{D}_{m} \rightarrow \operatorname{Sym}^{\ell}(\Sigma) \quad \text { s.t. } \Psi\left(v_{i}\right)=\mathbf{x}_{i}, \Psi\left(e_{i}\right) \subset \mathbb{T}_{i}, \quad i=1, \ldots, m
$$

is denoted by $\pi_{2}\left(\mathbf{x}_{1}, \ldots, \mathbf{x}_{m}\right)$. If $\pi_{2}\left(\mathbf{x}_{1}, \ldots, \mathbf{x}_{m}\right)$ is non-empty, [GW10, Proposition 3.3] implies that there is an affine correspondence

$$
\pi_{2}\left(\mathbf{x}_{1}, \ldots, \mathbf{x}_{m}\right) \simeq \operatorname{Ker}\left(\bigoplus_{i=1}^{m} \operatorname{Span}\left(\boldsymbol{\alpha}^{i}\right) \rightarrow \mathrm{H}_{1}(\Sigma ; \mathbb{Z})\right) \cong \mathrm{H}^{2}\left(W_{H}, \partial W_{H} ; \mathbb{Z}\right)
$$

where $\operatorname{Span}\left(\boldsymbol{\alpha}^{i}\right)$ denotes the submodule of $\mathrm{H}_{1}(\Sigma ; \mathbb{Z})$ spanned by the elements of $\boldsymbol{\alpha}^{i}$.

Fix intersection points $\mathbf{x}_{i}, \mathbf{x}_{i}^{\prime} \in \mathbb{T}_{i} \cap \mathbb{T}_{i+1}$ for $i=1, \ldots, m$. Two homotopy classes $\Psi \in$ $\pi_{2}\left(\mathbf{x}_{1}, \ldots, \mathbf{x}_{m}\right)$ and $\Psi^{\prime} \in \pi_{2}\left(\mathbf{x}_{1}^{\prime}, \ldots, \mathbf{x}_{m}^{\prime}\right)$ are called equivalent if there exist Whitney disks $\psi_{i} \in$ $\pi_{2}\left(\mathbf{x}_{i}, \mathbf{x}_{i}^{\prime}\right)$ for $i=1, \ldots, m$ so that $\Psi$ is obtained from $\Psi^{\prime}$ by juxtaposition of each disk $\psi_{i}$ at the vertex $\mathbf{x}_{i}^{\prime}$. Let Polygons $\{1, \ldots, m\}$ denote the set of equivalence classes of such $m$-gons. We may thus re-state Proposition 3.9 of [GW10] as follows.

Proposition 2.1. There is a one to one map

$$
\mathfrak{s}_{H}: \text { Polygons }_{\{1, \ldots, m\}} \longrightarrow \operatorname{Spin}^{c}\left(W_{H}\right)
$$

so that for any $\Psi \in \pi_{2}\left(\mathbf{x}_{1}, \ldots, \mathbf{x}_{m}\right)$, we have $\left.\mathfrak{s}_{H}([\Psi])\right|_{M_{i, i+1}}=\mathfrak{s}\left(\mathbf{x}_{i}\right)$ for any $i=1, \ldots, m$.

For every index set

$$
I=\left\{i_{1}<\cdots<i_{p}\right\} \subset\{1, \ldots, m\}
$$

with $|I| \geq 3$ we may consider the cobordism $W_{I}$ which corresponds to the compression of $W_{H}-$ $\coprod_{i \notin I}\left(U_{i} \times e_{i}\right)$ along the edges $\Sigma \times e_{i} \subset \Sigma \times \mathbb{D}_{m}$ with $i \notin I$. Thus, $W_{I}$ is represented by the Heegaard diagram $H_{I}=\left(\Sigma, \boldsymbol{\alpha}^{i_{1}}, \ldots, \boldsymbol{\alpha}^{i_{p}}, \mathbf{z}\right)$. Let

$$
r_{I}: \operatorname{Spin}^{c}\left(W_{H}\right) \rightarrow \operatorname{Spin}^{c}\left(W_{I}\right) .
$$

be the corresponding restriction map. Denote $\mathfrak{s}_{I}=r_{I}(\mathfrak{s})$ for any $\mathfrak{s} \in \operatorname{Spin}^{c}\left(W_{H}\right)$.

2.5. $\mathbb{A}$-diagrams and holomorphic polygon maps. Let $\mathbb{A}$ be a commutative algebra over $\mathbb{F}$ and $H=\left(\Sigma, \boldsymbol{\alpha}^{1}, \ldots, \boldsymbol{\alpha}^{m}, \mathbf{z}\right)$ be a balanced Heegaard diagram as before. Consider a map $\mathfrak{u}: \mathbf{z} \rightarrow \mathbb{A}$. We will denote $\mathfrak{u}\left(z_{i}\right)$ by $\mathfrak{u}_{i}$. For any 2 -chain $\mathcal{D}$ on $\Sigma$ with $\partial \mathcal{D}$ on $\boldsymbol{\alpha}^{1} \cup \cdots \cup \boldsymbol{\alpha}^{m}$ we define $\mathfrak{u}(\mathcal{D})$ as in Equation (1). Suppose $\Sigma \backslash \boldsymbol{\alpha}^{i}=\amalg_{j=1}^{m^{i}} A_{j}^{i}$. Then, as before, $\mathfrak{u}$ is called an $\mathbb{A}$-coloring for $H$ if the following are satisfied:

(1) For any $i, j \in\{1, \ldots, m\}$ we have $\sum_{k=1}^{m^{i}} \mathfrak{u}\left(A_{k}^{i}\right)=\sum_{l=1}^{m^{j}} \mathfrak{u}\left(A_{l}^{j}\right)$.

(2) If $A_{j}^{i}$ is not a punctured sphere, then $\mathfrak{u}\left(A_{j}^{i}\right)=0$.

Definition 2.6. Let the balanced Heegaard diagram $H=\left(\Sigma, \boldsymbol{\alpha}^{1}, \ldots, \boldsymbol{\alpha}^{m}, \mathbf{z}\right)$, the $\mathbb{A}$-coloring $\mathfrak{u}$ : $\mathbf{z} \rightarrow \mathbb{A}$ and $\operatorname{Spin}^{c}$ structure $\mathfrak{s} \in \operatorname{Spin}^{c}\left(W_{H}\right)$ be as above. We call

$$
\mathcal{H}=\left(\Sigma, \boldsymbol{\alpha}^{1}, \ldots, \boldsymbol{\alpha}^{m}, \mathfrak{u}: \mathbf{z} \rightarrow \mathbb{A}, \mathfrak{s}\right)
$$

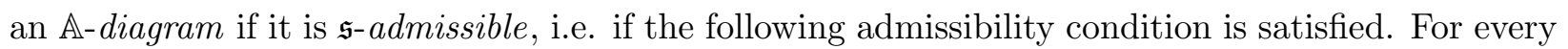
index set $I=\left\{i_{1}<\cdots<i_{p}\right\}$ and every doubly periodic domain

$$
\mathcal{P}=\mathcal{P}_{i_{1} i_{2}}+\mathcal{P}_{i_{2} i_{3}}+\cdots+\mathcal{P}_{i_{p} i_{1}}
$$


with $\mathcal{P}_{i j}$ a periodic domain on $\left(\Sigma, \boldsymbol{\alpha}^{i}, \boldsymbol{\alpha}^{j}, \mathbf{z}\right)$, the following is true. If

$$
\sum_{j=1}^{p}\left\langle c_{1}\left(\mathfrak{s}_{i_{j} i_{j+1}}\right), H\left(\mathcal{P}_{i_{j} i_{j+1}}\right)\right\rangle=0
$$

then either $\mathfrak{u}(\mathcal{P})=0$ in $\mathbb{A}$ or the coefficient of the domain $\mathcal{P}$ at some point $w$ is negative. Here, $\mathfrak{s}_{i j}$ is the $\operatorname{Spin}^{c}$ structure on the 3-manifold $M_{i j}$ (corresponding to $\left(\Sigma, \boldsymbol{\alpha}^{i}, \boldsymbol{\alpha}^{j}, \mathbf{z}\right)$ ) which is determined by $\mathfrak{s}$.

Note that the admissibility condition only depends on the restrictions $\mathfrak{s}_{i j}$ of $\mathfrak{s}$. In particular, for every family $\mathfrak{T}$ of $\operatorname{Spin}^{c}$ structures with the same restrictions $\mathfrak{s}_{i j}$, we can speak of $\mathbb{A}$-diagrams

$$
\left(\Sigma, \boldsymbol{\alpha}^{1}, \ldots, \boldsymbol{\alpha}^{m}, \mathfrak{u}: \mathbf{z} \rightarrow \mathbb{A}, \mathfrak{T}\right) .
$$

Let $\mathcal{H}=\left(\Sigma, \boldsymbol{\alpha}^{1}, \ldots, \boldsymbol{\alpha}^{m}, \mathfrak{u}, \mathfrak{s}\right)$ be an $\mathbb{A}$-diagram and choose an appropriate translation invariant family of generic almost complex structure $J=\left\{J_{z}\right\}_{z \in \mathbb{D}_{m}}$. For every pair of indices $i<j$ we may thus define the chain complex $\mathrm{CF}_{J}\left(\Sigma, \boldsymbol{\alpha}^{i}, \boldsymbol{\alpha}^{j}, \mathfrak{u}, \mathfrak{s}_{i j}\right)$. Associated with any subset

$$
I=\left\{i_{1}<\cdots<i_{p}\right\} \subset\{1, \ldots, m\}
$$

of indices, we may then define a holomorphic polygon map

$$
\begin{gathered}
\mathfrak{f}_{I}: \bigotimes_{j=1}^{p-1} \mathrm{CF}_{J}\left(\Sigma, \boldsymbol{\alpha}^{i_{j}}, \boldsymbol{\alpha}^{i_{j+1}}, \mathfrak{u}, \mathfrak{s}_{i_{j} i_{j+1}}\right) \longrightarrow \mathrm{CF}_{J}\left(\Sigma, \boldsymbol{\alpha}^{i_{1}}, \boldsymbol{\alpha}^{i_{p}}, \mathfrak{u}, \mathfrak{s}_{i_{1} i_{p}}\right) . \\
\mathfrak{f}_{I}\left(\mathbf{x}_{1} \otimes \mathbf{x}_{2} \otimes \cdots \otimes \mathbf{x}_{p-1}\right):=\sum_{\substack{\mathbf{x}_{p} \in \mathbb{T}_{i_{1}} \cap \mathbb{T}_{i_{p}} \\
\mathfrak{s}\left(\mathbf{x}_{p}\right)=\mathfrak{s}_{i_{1} i_{p}}}} \sum_{\substack{\Psi \in \pi_{2}\left(\mathbf{x}_{1}, \mathbf{x}_{2}, \ldots, \mathbf{x}_{p}\right) \\
\mu(\Psi)=3-p \\
[\Psi]=\mathfrak{s}_{I}}}(\mathfrak{m}(\Psi) \mathfrak{u}(\Psi)) \cdot \mathbf{x}_{p}
\end{gathered}
$$

where $\mu(\Psi)$ denotes the Maslov index of the polygon class $\Psi$ and $\mathfrak{m}(\Psi)$ is the count of points in $\mathcal{M}(\Psi)$ modulo 2 . 


\section{TANGle COMPleX AND the issue of NATURALity}

In this section we address the issue of naturality in the sense of [JT] for the construction of [AE15]. More precisely, we strengthen our result in [AE15] and show that for any $\mathbb{A}$-module $\mathbb{M}$, $H^{\mathbb{M}}$ defines a functor from $\mathbb{A}$-Tang (See Definition 1.4) to $\mathbb{A}$-Mod. For the most part, the argument of [JT] for the naturality of sutured Floer homology may be copied here without significant modifications. In fact, we need to go through the argument presented in Section 9 of [JT] and check that all statements remain valid, a task that is outlined in the present section.

3.1. Oriented graph of isotopy Heegaard diagrams. Let us fix a commutative algebra $\mathbb{A}$ over $\mathbb{F}$ and an $\mathbb{A}$-module $\mathbb{M}$ as before. Consider an $\mathbb{A}$-tangle $\mathcal{T}=[M, T, \mathfrak{s}, \mathfrak{u}]$ and let $\mathcal{H}=(\Sigma, \boldsymbol{\alpha}, \boldsymbol{\beta}, \mathfrak{u}, \mathfrak{s})$ be a Heegaard diagram for $\mathcal{T}$. Any collection of pairwise disjoint circles on $\Sigma$ (like $\boldsymbol{\alpha}$ and $\boldsymbol{\beta}$ ) is called an attaching set. Recall that each attaching set $\gamma$ on the pointed surface $(\Sigma, \mathbf{z})$ determines a tangle $(C[\gamma], T[\gamma])$ where $C[\gamma]$ is the compression body obtained from $\Sigma \times[0,1]$ by attaching 2-handles along $\gamma \times\{1\}$ and $T[\gamma]=\mathbf{z} \times[0,1]$. Then, $\partial^{-} C[\gamma]=\Sigma \times\{0\} \cong \Sigma$ and $\partial^{+} C[\gamma] \cong \Sigma[\gamma]$, where $\Sigma[\gamma]$ denotes the surface obtained by performing surgery on $\Sigma$ along the curves in $\gamma$. Attaching sets $\gamma$ and $\gamma^{\prime}$ on $\Sigma$ are called compression equivalent, denoted by $\gamma \sim \gamma^{\prime}$, if there is a diffeomorphism $d:(C[\gamma], T[\gamma]) \rightarrow$ $\left(C\left[\gamma^{\prime}\right], T\left[\gamma^{\prime}\right]\right)$ such that it is the identity on $(\Sigma, \mathbf{z})=\left(\partial^{-} C[\gamma], \partial^{-} T[\gamma]\right)=\left(\partial^{-} C\left[\gamma^{\prime}\right], \partial^{-} T\left[\gamma^{\prime}\right]\right)$. This gives an equivalence relation which descends to the isotopy classes, denoted by $[\gamma]$, of attaching sets $\gamma$ on $\Sigma$. If $\gamma \sim \gamma^{\prime}$ then $[\gamma]$ and $\left[\gamma^{\prime}\right]$ are related by a sequence of handle slides, [JT, Lemma $2.11]$.

If for the attaching sets $\boldsymbol{\alpha}^{\prime}$ and $\boldsymbol{\beta}^{\prime}$ we have $[\boldsymbol{\alpha}]=\left[\boldsymbol{\alpha}^{\prime}\right]$ and $[\boldsymbol{\beta}]=\left[\boldsymbol{\beta}^{\prime}\right]$, and the $\mathfrak{s}$-admissibility condition is satisfied then $\left(\Sigma, \boldsymbol{\alpha}^{\prime}, \boldsymbol{\beta}^{\prime}, \mathfrak{u}, \mathfrak{s}\right)$ is also a Heegaard diagram for $\mathcal{T}$. We may thus refer to the set of all such Heegaard diagrams as an isotopy diagram $(\Sigma, A, B, \mathfrak{u}, \mathfrak{s})$ for $\mathcal{T}$, where $A=[\boldsymbol{\alpha}]$ and $B=[\boldsymbol{\beta}]$.

The isotopy diagrams

$$
\mathcal{H}_{1}=\left(\Sigma_{1}, A_{1}, B_{1}, \mathfrak{u}_{1}: \mathbf{z}_{1} \rightarrow \mathbb{A}, \mathfrak{s}_{1}\right) \text { and } \mathcal{H}_{2}=\left(\Sigma_{2}, A_{2}, B_{2}, \mathfrak{u}_{2}: \mathbf{z}_{2} \rightarrow \mathbb{A}, \mathfrak{s}_{2}\right)
$$

are called $\alpha$-equivalent if $\Sigma_{1}=\Sigma_{2}, \mathbf{z}_{1}=\mathbf{z}_{2}, \mathfrak{u}_{1}=\mathfrak{u}_{2}$ and $B_{1}=B_{2}$ while $A_{1} \sim A_{2}$ and under the natural correspondence between $\operatorname{Spin}^{c}$ structures, $\mathfrak{s}_{1}=\mathfrak{s}_{2}$. Similarly, we may define $\beta$-equivalence. Stabilization and destabilization also induce operations on isotopy diagrams. Furthermore, a diffeomorphism from $\mathcal{H}_{1}$ to $\mathcal{H}_{2}$ is an orientation preserving diffeomorphism $d: \Sigma_{1} \rightarrow \Sigma_{2}$ such that $d\left(A_{1}\right)=A_{2}, d\left(B_{1}\right)=B_{2}, d\left(\mathbf{z}_{1}\right)=\mathbf{z}_{2}, d^{*} \mathfrak{s}_{2}=\mathfrak{s}_{1}$.

Finally, we may define the oriented graph $\mathcal{G}=\mathcal{G}_{\mathcal{T}}$ (in the sense of [JT, Definition 2.21]) as follows. The vertices of $\mathcal{G}$ are isotopy diagrams for $\mathcal{T}$ and for any two vertices $\mathcal{H}_{1}$ and $\mathcal{H}_{2}$, the set of edges connecting $\mathcal{H}_{1}$ to $\mathcal{H}_{2}$, denoted by $\mathcal{G}\left(\mathcal{H}_{1}, \mathcal{H}_{2}\right)$, is a union of four sets

$$
\mathcal{G}\left(\mathcal{H}_{1}, \mathcal{H}_{2}\right)=\mathcal{G}_{\alpha}\left(\mathcal{H}_{1}, \mathcal{H}_{2}\right) \amalg \mathcal{G}_{\beta}\left(\mathcal{H}_{1}, \mathcal{H}_{2}\right) \amalg \mathcal{G}_{\text {stab }}\left(\mathcal{H}_{1}, \mathcal{H}_{2}\right) \amalg \mathcal{G}_{\text {diff }}\left(\mathcal{H}_{1}, \mathcal{H}_{2}\right),
$$

defined as follows. The set $\mathcal{G}_{\alpha}\left(\mathcal{H}_{1}, \mathcal{H}_{2}\right)\left(\right.$ or $\left.\mathcal{G}_{\beta}\left(\mathcal{H}_{1}, \mathcal{H}_{2}\right)\right)$ consists of a single arrow if $\mathcal{H}_{1}$ and $\mathcal{H}_{2}$ are $\alpha$-equivalent (or $\beta$-equivalent), otherwise it is empty. Similarly, $\mathcal{G}_{\text {stab }}\left(\mathcal{H}_{1}, \mathcal{H}_{2}\right)$ consists of a single arrow if $\mathcal{H}_{2}$ is obtained from $\mathcal{H}_{1}$ by a stabilization or destabilization and $\mathcal{G}_{\text {diff }}\left(\mathcal{H}_{1}, \mathcal{H}_{2}\right)$ contains an arrow corresponding to any diffeomorphism from $\mathcal{H}_{1}$ to $\mathcal{H}_{2}$. Let $\mathcal{G}_{\alpha}, \mathcal{G}_{\beta}, \mathcal{G}_{\text {stab }}$ and $\mathcal{G}_{\text {diff denote }}$ the corresponding sub-graphs of $\mathcal{G}$. The sub-graphs $\mathcal{G}_{\alpha}, \mathcal{G}_{\beta}$ and $\mathcal{G}_{\text {diff }}$ are in fact categories when endowed with the obvious compositions. The graph $\mathcal{G}$ is connected as an oriented graph.

3.2. Special Heegaard diagrams. A Heegaard diagram $H=(\Sigma, \boldsymbol{\alpha}, \boldsymbol{\beta}, \mathbf{z})$ is called special if $\boldsymbol{\alpha} \sim \boldsymbol{\beta}$, i.e. $\boldsymbol{\alpha}$ is compression equivalent to $\boldsymbol{\beta}$. Any special Heegaard diagram determines a balanced tangle $(M, T)$ that is obtained from a product tangle by applying sugeries on $\ell 0$-dimensional spheres, where $\ell=|\boldsymbol{\alpha}|=|\boldsymbol{\beta}|$. Furthermore, if a balanced tangle $(M, T)$ has a special Heegaard diagram then $\operatorname{HF}(M, T, \mathfrak{s})=0$ for any non-torsin class $\mathfrak{s} \in \operatorname{Spin}^{c}(M)$. Recall that a $\operatorname{Spin}^{c}$ class $\mathfrak{s} \in \operatorname{Spin}^{c}(M)$ is called torsion if $\mathfrak{s}=[\mathfrak{s}]$ for a torsion relative $\operatorname{Spin}^{c}$ class $\mathfrak{s} \in \operatorname{Spin}^{c}(M, T)$ i.e. $c_{1}(\underline{\mathfrak{s}})=0$ as an element of $\mathrm{H}^{2}(X, \mathbb{Z})$ for $X=M-\operatorname{nd}(T)$.

Associated with every $z_{i} \in \mathbf{z}=\left\{z_{1}, \ldots, z_{\kappa}\right\}$, let $\mu_{i}$ denote a small simple closed curve which is the boundary of a small disk around $z_{i} \in \Sigma$. The torsion relative $\operatorname{Spin}^{c}$ structures corresponding to 
a special Heegaard diagram are related to each other by adding integer multiples of the cohomology classes $\mathrm{PD}\left[\mu_{i}\right] \in \mathrm{H}^{2}(M, T ; \mathbb{Z})$, for $i=1, \ldots, \kappa$. In fact, the torsion relative $\operatorname{Spin}^{c}$ structures $\underline{\mathfrak{s}} \in$ $\operatorname{Spin}^{c}(M, T)$ with the property that $\operatorname{HF}(M, T, \mathfrak{s})$ is non-zero form a cone, in the sense that every such relative $\operatorname{Spin}^{c}$ structure $\underline{\mathfrak{s}}$ is of the form

$$
\underline{\mathfrak{s}}=\underline{\mathfrak{s}}_{0}+\sum_{i=1}^{\kappa} a_{i} \mathrm{PD}\left[\mu_{i}\right], \quad \text { where } a_{1}, \ldots, a_{\kappa} \in \mathbb{Z}^{\geq 0} .
$$

The relative $\operatorname{Spin}^{c}$ structure $\underline{\mathfrak{s}}_{0} \in \operatorname{Spin}^{c}(M, T)$ is thus uniquely associated with our special Heegaard diagram, and is called its distinguished (torsion) relative $\operatorname{Spin}^{c}$ structure for the special Heegaard diagram $H$.

Fix a special Heegaard diagram $H=(\Sigma, \boldsymbol{\alpha}, \boldsymbol{\beta}, \mathbf{z})$ as above, and let $(M, T)$ be the corresponding balanced tangle. Further, assume that for the torsion $\operatorname{Spin}^{c}$ class $\mathfrak{s}_{0} \in \operatorname{Spin}^{c}(M, T)$ the diagram $H$ is $\mathfrak{s}_{0}$-admissible. If $\mathbf{z}=\left\{z_{1}, \ldots, z_{\kappa}\right\}$, then $\mathbb{A}_{T}=\frac{\mathbb{Z}\left[\mathfrak{u}_{1}, \ldots, \mathfrak{u}_{\kappa}\right]}{\mathcal{I}}$ where $\mathfrak{u}_{i}$ is the variable corresponding to $z_{i}$ and $\mathcal{I}$ is the ideal of relations. Note that $\mathcal{I}$ is generated by monomials corresponding to connected components of $\Sigma \backslash \boldsymbol{\alpha}$ (or $\Sigma \backslash \boldsymbol{\beta}$ ) that are not punctured spheres.

Consider $a \cdot \mathbf{x}, b \cdot \mathbf{y} \in \operatorname{CF}(M, T, \underline{\mathfrak{s}})$ such that $\mathbf{x}, \mathbf{y} \in \mathbb{T}_{\alpha} \cap \mathbb{T}_{\beta}, \underline{\mathfrak{s}} \in \operatorname{Spin}^{c}(M, T)$ is torsion and

$$
a=\mathfrak{u}_{1}^{a_{1}} \cdots \mathfrak{u}_{\kappa}^{a_{\kappa}} \quad \text { and } \quad b=\mathfrak{u}_{1}^{b_{1}} \cdots \mathfrak{u}_{\kappa}^{b_{\kappa}}
$$

are non-zero monomials in $\mathbb{A}_{T}$. Then, we say a homotopy disk $\phi \in \pi_{2}(\mathbf{x}, \mathbf{y})$ connects $a \cdot \mathbf{x}$ to $b \cdot \mathbf{y}$ if $a_{i}+n_{z_{i}}(\phi)=b_{i}$ for any $i=1, \ldots, \kappa$. We define $\operatorname{gr}(a \cdot \mathbf{x}, b \cdot \mathbf{y})=\mu(\phi)$ for a homotopy disk $\phi \in \pi_{2}(\mathbf{x}, \mathbf{y})$ connecting $a \cdot \mathbf{x}$ to $b \cdot \mathbf{y}$.

Lemma 3.1. The map gr is well-defined and induces a relative $\mathbb{Z}$-grading on

$$
\mathrm{CF}(M, T, \underline{\mathfrak{s}})=\mathrm{CF}(\Sigma, \boldsymbol{\alpha}, \boldsymbol{\beta}, \mathbf{z}, \underline{\mathfrak{s}}),
$$

for every torsion relative $\operatorname{Spin}^{c}$ structure $\underline{\mathfrak{s}} \in \operatorname{Spin}^{c}(M, T)$. Furthermore, the subgroup of $\operatorname{HF}\left(M, T, \underline{\mathfrak{s}}_{0}\right)$ in top homological grading is isomorphic to $\mathbb{F}$ for the distinguished relative $\operatorname{Spin}^{c}$ structure $\underline{\mathfrak{s}}_{0} \in$ $\operatorname{Spin}^{c}(M, T)$.

Proof. Let $\phi_{1}, \phi_{2} \in \pi_{2}(\mathbf{x}, \mathbf{y})$ be homotopy disks connecting $a . \mathbf{x}$ to $b . \mathbf{y}$. Then, $\mathcal{P}=\phi_{1}-\phi_{2}$ would be a periodic domain such that $n_{z_{i}}(\mathcal{P})=0$ for any $i=1, \ldots, \kappa$. Hence,

$$
\mu\left(\phi_{1}\right)-\mu\left(\phi_{2}\right)=\mu(\mathcal{P})=\left\langle c_{1}(\underline{\mathfrak{s}}), H(\mathcal{P})\right\rangle=0 .
$$

In particular, gr is well-defined. For the second part, it is not hard to show that $\operatorname{HF}\left(M, T, \underline{\mathfrak{s}}_{0}\right)$ is invariant up to isomorphism in each relative grading. So the discussions in Section 6.2 of [AE15] implies that the subgroup with the top grading is isomorphic to $\mathbb{F}$.

Hence, the homology group in the top grading has a well-defined generator up to sign, called top generator, in the summand $\operatorname{HF}\left(M, T, \underline{\mathfrak{s}}_{0}\right)$ of $\operatorname{HF}\left(M, T, \mathfrak{s}_{0}\right)$, where $\mathfrak{s}_{0} \in \operatorname{Spin}^{c}(M, T)$ is the distinguished torsion $\operatorname{Spin}^{c}$ structure and $\mathfrak{s}_{0}=\left[\underline{\mathfrak{s}}_{0}\right]$ is its image in $\operatorname{Spin}^{c}(M)$. This generator is denoted by $\Theta_{\alpha \beta}$.

3.3. Weak Heegaard invariance. In this section, we check that $\mathrm{HF}^{\mathbb{M}}$ is a weak Heegaard invariant i.e. for any $\mathbb{A}$-tangle $\mathcal{T}$, we construct a well-defined $\mathbb{A}$-module $\operatorname{HF}^{\mathbb{M}}(\mathcal{H})$ for every vertex $\mathcal{H}$ of $\mathcal{G}_{\mathcal{T}}$ and associate an isomorphism to any edge of $\mathcal{G}_{\mathcal{T}}$.

Consider an $\mathbb{A}$-diagram $\mathcal{H}=(\Sigma, \boldsymbol{\alpha}, \boldsymbol{\beta}, \mathfrak{u}, \mathfrak{s})$ and a complex structure $\mathfrak{j}$ on $\Sigma$. Recall that any generic path $J_{s}$ of perturbations of $\operatorname{Sym}^{\ell}(\mathfrak{j})$ gives a chain complex

$$
\mathrm{CF}_{J_{s}}(\Sigma, \boldsymbol{\alpha}, \boldsymbol{\beta}, \mathfrak{u}, \mathfrak{s}) \text {, }
$$

generated by the intersection points $\mathbf{x} \in \mathbb{T}_{\alpha} \cap \mathbb{T}_{\beta}$ with $\mathfrak{s}(\mathbf{x})=\mathfrak{s}$, where $\ell=|\boldsymbol{\alpha}|=|\boldsymbol{\beta}|$. We will usually drop the complex structure $\mathfrak{j}$ on $\Sigma$ from the notation. Given two different choices $\left(\mathfrak{j}, J_{s}\right)$ and $\left(\mathfrak{j}^{\prime}, J_{s}^{\prime}\right)$, the proof of Lemma 2.11 from [OS06] gives an isomorphism of $\mathbb{A}$-modules

$$
\Phi_{J_{s} \rightarrow J_{s}^{\prime}}: \operatorname{HF}_{J_{s}}^{\mathbb{M}}(\Sigma, \boldsymbol{\alpha}, \boldsymbol{\beta}, \mathfrak{u}, \mathfrak{s}) \longrightarrow \operatorname{HF}_{J_{s}^{\prime}}^{\mathbb{M}}(\Sigma, \boldsymbol{\alpha}, \boldsymbol{\beta}, \mathfrak{u}, \mathfrak{s}) .
$$


for any $\mathbb{A}$-module $\mathbb{M}$. Moreover,

$$
\Phi_{J_{s}^{\prime} \rightarrow J_{s}^{\prime \prime}} \circ \Phi_{J_{s} \rightarrow J_{s}^{\prime}}=\Phi_{J_{s} \rightarrow J_{s}^{\prime \prime}}
$$

One may thus define

$$
\operatorname{HF}^{\mathbb{M}}(\Sigma, \boldsymbol{\alpha}, \boldsymbol{\beta}, \mathfrak{u}, \mathfrak{s})=\frac{\coprod_{J_{s}} \operatorname{HF}_{J_{s}}^{\mathbb{M}}(\Sigma, \boldsymbol{\alpha}, \boldsymbol{\beta}, \mathfrak{u}, \mathfrak{s})}{\sim}
$$

where $x \sim y$ if $y=\Phi_{J_{s} \rightarrow J_{s}^{\prime}}(x)$ for some $J_{s}$ and $J_{s}^{\prime}$. Since we will face several equivalence relations in the definition of $\mathrm{HF}^{\mathbb{M}}(\mathcal{T})$ we will abuse the notation and denote all of them by $\sim$, leaving it to the reader to make the distinctions.

Let us ssume that

$$
\mathcal{H}=\left(\Sigma, \boldsymbol{\alpha}, \boldsymbol{\beta}, \boldsymbol{\beta}^{\prime}, \mathfrak{u}, \mathfrak{t}\right)
$$

is an $\mathbb{A}$-diagram and $\boldsymbol{\beta} \sim \boldsymbol{\beta}^{\prime}$. Furthermore, suppose that the restriction of $\mathfrak{t}$ to $M_{\beta \beta^{\prime}}$ is the torsion $\operatorname{Spin}^{c}$ structure $\mathfrak{s}_{0}$, which was discussed in the previous subsection. With this restriction in place, $\mathfrak{t}$ is determined by its restriction $\mathfrak{s}$ to $M_{\alpha \beta}$, and the induced $\operatorname{Spin}^{c}$ structure on $M_{\alpha \beta^{\prime}}$ is in correspondence with $\mathfrak{s}$ under the natural identification of $M_{\alpha \beta}$ with $M_{\alpha \beta^{\prime}}$. We will thus abuse the notation and denote the above $\mathbb{A}$-diagram by

$$
\left(\Sigma, \boldsymbol{\alpha}, \boldsymbol{\beta}, \boldsymbol{\beta}^{\prime}, \mathfrak{u}, \mathfrak{s}\right)
$$

implying that the above restrictions on $\mathfrak{t}$ are imposed. Then, using the top generator $\Theta_{\beta \beta^{\prime}}$ we may define an isomorphism

$$
\Phi_{\beta \rightarrow \beta^{\prime}}^{\alpha}: \operatorname{HF}^{\mathbb{M}}(\Sigma, \boldsymbol{\alpha}, \boldsymbol{\beta}, \mathfrak{u}, \mathfrak{s}) \longrightarrow \operatorname{HF}^{\mathbb{M}}\left(\Sigma, \boldsymbol{\alpha}, \boldsymbol{\beta}^{\prime}, \mathfrak{u}, \mathfrak{s}\right) .
$$

The arguments in the sequence of lemmas preceding Proposition 9.9 in [JT] may then be copied in our setup to show that if $(\Sigma, \boldsymbol{\alpha}, \boldsymbol{\beta}, \mathfrak{u}, \mathfrak{s})$ and $\left(\Sigma, \boldsymbol{\alpha}, \boldsymbol{\beta}^{\prime}, \mathfrak{u}, \mathfrak{s}\right)$ are Heegaard diagrams for $\mathcal{T}$, one may define a chain map and a well-defined induced isomorphism

$$
\Phi_{\beta \rightarrow \beta^{\prime}}^{\alpha}: \operatorname{HF}^{\mathbb{M}}(\Sigma, \boldsymbol{\alpha}, \boldsymbol{\beta}, \mathfrak{u}, \mathfrak{s}) \longrightarrow \operatorname{HF}^{\mathbb{M}}\left(\Sigma, \boldsymbol{\alpha}, \boldsymbol{\beta}^{\prime}, \mathfrak{u}, \mathfrak{s}\right),
$$

using the top generators and a detour to admissible triple diagrams (note that the above assumptions do not imply that the $\left(\Sigma, \boldsymbol{\alpha}, \boldsymbol{\beta}, \boldsymbol{\beta}^{\prime}, \mathfrak{u}, \mathfrak{s}\right)$ is an $\mathbb{A}$-diagram). Furthermore, if $\left(\Sigma, \boldsymbol{\alpha}, \boldsymbol{\beta}^{\prime \prime}, \mathfrak{u}, \mathfrak{s}\right)$ is a third Heegaard diagram for $\mathcal{T}$ so that $\boldsymbol{\beta} \sim \boldsymbol{\beta}^{\prime} \sim \boldsymbol{\beta}^{\prime \prime}$, we get

$$
\Phi_{\beta^{\prime} \rightarrow \beta^{\prime \prime}}^{\alpha} \circ \Phi_{\beta \rightarrow \beta^{\prime}}^{\alpha}=\Phi_{\beta \rightarrow \beta^{\prime \prime}}^{\alpha} .
$$

Similarly, one may define $\Phi_{\beta}^{\alpha \rightarrow \alpha^{\prime}}$. If $(\Sigma, \boldsymbol{\alpha}, \boldsymbol{\beta}, \mathfrak{u}, \mathfrak{s}),\left(\Sigma, \boldsymbol{\alpha}^{\prime}, \boldsymbol{\beta}, \mathfrak{u}, \mathfrak{s}\right),\left(\Sigma, \boldsymbol{\alpha}, \boldsymbol{\beta}^{\prime}, \mathfrak{u}, \mathfrak{s}\right)$ and $\left(\Sigma, \boldsymbol{\alpha}^{\prime}, \boldsymbol{\beta}^{\prime}, \mathfrak{u}, \mathfrak{s}\right)$ are Heegaard diagrams for $\mathcal{T}$ so that $\boldsymbol{\alpha} \sim \boldsymbol{\alpha}^{\prime}$ and $\boldsymbol{\beta} \sim \boldsymbol{\beta}^{\prime}$, one may define

$$
\Phi_{\beta \rightarrow \beta^{\prime}}^{\alpha \rightarrow \alpha^{\prime}}=\Phi_{\beta \rightarrow \beta^{\prime}}^{\alpha^{\prime}} \circ \Phi_{\beta}^{\alpha \rightarrow \alpha^{\prime}}=\Phi_{\beta^{\prime}}^{\alpha \rightarrow \alpha^{\prime}} \circ \Phi_{\beta \rightarrow \beta^{\prime}}^{\alpha} .
$$

It follows from [JT, Lemma 9.11] that these isomorphisms satisfy

$$
\Phi_{\beta^{\prime} \rightarrow \beta^{\prime \prime}}^{\alpha^{\prime} \rightarrow \alpha^{\prime \prime}} \circ \Phi_{\beta \rightarrow \beta^{\prime}}^{\alpha \rightarrow \alpha^{\prime}}=\Phi_{\beta \rightarrow \beta^{\prime \prime}}^{\alpha \rightarrow \alpha^{\prime \prime}} \quad \text { and } \quad \Phi_{\beta \rightarrow \beta}^{\alpha \rightarrow \alpha}=\Phi_{\beta \rightarrow \beta}^{\alpha}=\Phi_{\beta}^{\alpha \rightarrow \alpha}=\operatorname{Id}_{\mathrm{HF}^{\mathbb{M}}(\Sigma, \alpha, \beta, \mathfrak{u}, \mathfrak{s})} .
$$

These isomorphisms may be used to construct a well-defined $\mathbb{A}$-module $\operatorname{HF}^{\mathbb{M}}(\mathcal{H})$ for every vertex $\mathcal{H}$ of $\mathcal{G}_{\mathcal{T}}$ and associate isomorphisms to edges of $\mathcal{G}_{\alpha}$ and $\mathcal{G}_{\beta}$, as follows.

Given an isotopy diagram $\mathcal{H}=(\Sigma, A, B, \mathfrak{u}, \mathfrak{s})$ for $\mathcal{T}$, we denote by $\mathcal{M}_{\mathcal{H}}$ the set of all Heegaard diagrams corresponding to $\mathcal{H}$, and we let

$$
\operatorname{HF}^{\mathbb{M}}(\mathcal{H})=\frac{\coprod_{(\Sigma, \alpha, \beta, \mathfrak{u}, \mathfrak{s}) \in \mathcal{M}_{\mathcal{H}}} \operatorname{HF}^{\mathbb{M}}(\Sigma, \boldsymbol{\alpha}, \boldsymbol{\beta}, \mathfrak{u}, \mathfrak{s})}{\sim}
$$

where the generators $x \in \operatorname{HF}^{\mathbb{M}}(\Sigma, \boldsymbol{\alpha}, \boldsymbol{\beta}, \mathfrak{u}, \mathfrak{s})$ and $x^{\prime} \in \operatorname{HF}^{\mathbb{M}}\left(\Sigma, \boldsymbol{\alpha}^{\prime}, \boldsymbol{\beta}^{\prime}, \mathfrak{u}, \mathfrak{s}\right)$ are equivalent if and only if $x^{\prime}=\Phi_{\beta \rightarrow \beta^{\prime}}^{\alpha \rightarrow \alpha^{\prime}}(x)$.

Suppose $\mathcal{H}=(\Sigma, A, B, \mathfrak{u}, \mathfrak{s})$ and $\mathcal{H}^{\prime}=\left(\Sigma, A, B^{\prime}, \mathfrak{u}, \mathfrak{s}\right)$ are $\beta$-equivalent. Pick representatives $(\Sigma, \boldsymbol{\alpha}, \boldsymbol{\beta}, \mathfrak{u}, \mathfrak{s})$ and $\left(\Sigma, \boldsymbol{\alpha}, \boldsymbol{\beta}^{\prime}, \mathfrak{u}, \mathfrak{s}\right)$ of $\mathcal{H}$ and $\mathcal{H}^{\prime}$, respectively. The formal proof of [JT, Lemma 9.17] then implies that $\Phi_{\beta \rightarrow \beta^{\prime}}^{\alpha}$ descends to a well-defined isomorphism

$$
\Phi_{B \rightarrow B^{\prime}}^{A}: \operatorname{HF}^{\mathbb{M}}(\mathcal{H}) \longrightarrow \operatorname{HF}^{\mathbb{M}}\left(\mathcal{H}^{\prime}\right) .
$$


Similarly, an $\alpha$-equivalence from $\mathcal{H}=(\Sigma, A, B, \mathfrak{u}, \mathfrak{s})$ to $\mathcal{H}^{\prime}=\left(\Sigma, A^{\prime}, B, \mathfrak{u}, \mathfrak{s}\right)$ gives a well-defined isomorphism

$$
\Phi_{B}^{A \rightarrow A^{\prime}}: \operatorname{HF}^{\mathbb{M}}(\mathcal{H}) \longrightarrow \operatorname{HF}^{\mathbb{M}}\left(\mathcal{H}^{\prime}\right) .
$$

Consider an edge of $\mathcal{G}_{\text {diff }}$ i.e. a diffeomorphism $d: \mathcal{H} \rightarrow \mathcal{H}^{\prime}$. It gives a correspondence between complex structures, which in turn gives the isomorphism

$$
d_{*}: \operatorname{HF}^{\mathbb{M}}(\mathcal{H}) \longrightarrow \operatorname{HF}^{\mathbb{M}}\left(\mathcal{H}^{\prime}\right),
$$

c.f. [JT, Definition 9.19 and Lemma 9.20].

Finally, if the Heegaard diagram $\left(\Sigma^{\prime}, \boldsymbol{\alpha}^{\prime}, \boldsymbol{\beta}^{\prime}, \mathfrak{u}^{\prime}, \mathfrak{s}^{\prime}\right)$ is obtained from $(\Sigma, \boldsymbol{\alpha}, \boldsymbol{\beta}, \mathfrak{u}, \mathfrak{s})$ by stabilization, there is a correspondence between the homotopy classes of disks on the two sides. Furthermore, for suitable almost complex structures on the two diagrams, there is an isomorphism of chain complexes associated with the two diagrams. If $\mathcal{H}$ and $\mathcal{H}^{\prime}$ denote the isotopy diagrams corresponding to the above two Heegaard diagrams for $\mathcal{T}$, [JT, Lemma 9.21] (which is basically [OS06, Lemma 2.15]) may be used to construct the isomorphism

$$
\sigma_{\mathcal{H} \rightarrow \mathcal{H}^{\prime}}: \operatorname{HF}^{\mathbb{M}}(\mathcal{H}) \longrightarrow \operatorname{HF}^{\mathbb{M}}\left(\mathcal{H}^{\prime}\right) .
$$

Hence, $\mathrm{HF}^{\mathbb{M}}$ is a weak Heegaard invariant, and the above considerations reprove the invariance of the quasi-isomorphism type of the chain complex $\operatorname{CF}^{\mathbb{M}}(\mathcal{T})$.

3.4. Strong Heegaard invariance. The weak Heegaard invariant $\mathrm{HF}^{\mathbb{M}}$ is a strong Heegaard invariant if it satisfies the following axioms, $[\mathrm{JT}]$ :

(1) Fuctoriality: The restriction of $\mathrm{HF}^{\mathbb{M}}$ to the categories $\mathcal{G}_{\alpha}, \mathcal{G}_{\beta}$ and $\mathcal{G}_{\text {diff }}$ is a functor to the category $\mathbb{A}$-Mod of $\mathbb{A}$-modules. Moreover, if $\sigma: \mathcal{H} \rightarrow \mathcal{H}^{\prime}$ is a stabilization and $\sigma^{\prime}: \mathcal{H}^{\prime} \rightarrow \mathcal{H}$ is the corresponding destabilization then $\operatorname{HF}^{\mathbb{M}}\left(\sigma^{\prime}\right)=\operatorname{HF}^{\mathbb{M}}(\sigma)^{-1}$.

(2) Commutativity: For every distinguished rectangle

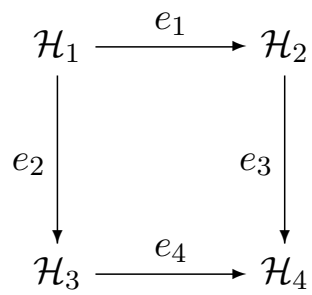

in $\mathcal{G}$ we have $\operatorname{HF}^{\mathbb{M}}\left(e_{3}\right) \circ \operatorname{HF}^{\mathbb{M}}\left(e_{1}\right)=\operatorname{HF}^{\mathbb{M}}\left(e_{4}\right) \circ \operatorname{HF}^{\mathbb{M}}\left(e_{2}\right)$, where $\operatorname{HF}^{\mathbb{M}}\left(e_{i}\right)$ denotes the associated isomorphism to $e_{i}$. Here, distinguished rectangles are the rectangles of the above type such that one of the following is the case:

- The horizontal arrows are $\alpha$-equivalences while the vertical arrows are $\beta$-equivalences.

- Both horizontal arrows are $\alpha$ - or $\beta$-equivalences, while the vertical arrows are stabilizations.

- Both horizontal arrows are $\alpha$ - or $\beta$ - equivalences, while the vertical arrows are diffeomorphisms with the same restriction on the surface.

- The square corresponds to the two possible ways of performing disjoint stabilizations.

- The horizontal arrows are diffeomorphisms and the vertical arrows are stabilizations which correspond to one another via the diffeomorphisms.

(3) Continuity: If $\mathcal{H}$ is a vertex of $\mathcal{G}$ and $d \in \mathcal{G}_{\text {diff }}(\mathcal{H}, \mathcal{H})$ is a diffeomorphism isotopic to the identity, then $\operatorname{HF}^{\mathbb{M}}(d)=\operatorname{Id}_{\mathrm{HF}^{\mathbb{M}}(\mathcal{H})}$.

(4) Handleswap invariance: For every simple handle swap

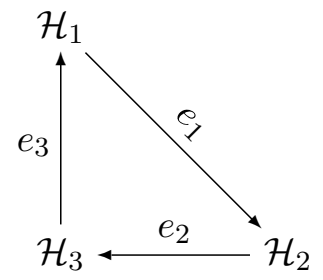


in the sense of [JT, Definition 2.32] in $\mathcal{G}$ we have

$$
\operatorname{HF}^{\mathbb{M}}\left(e_{3}\right) \circ \operatorname{HF}^{\mathbb{M}}\left(e_{2}\right) \circ \operatorname{HF}^{\mathbb{M}}\left(e_{1}\right)=\operatorname{Id}_{\mathrm{HF}^{\mathbb{M}}\left(\mathcal{H}_{1}\right)} .
$$

Functoriality follows from equations (2) and (3) for $\alpha$ - and $\beta$-equivalences, and is a consequence of the definition for the diffeomorphisms. Moreover, the equality $\operatorname{HF}_{\mathbb{M}}\left(\sigma^{\prime}\right)=\operatorname{HF}_{\mathbb{M}}(\sigma)^{-1}$ is a consequence of the definition.

Our earlier considerations proves the axiom of commutativity for the first three types of distinguished rectangles. The commutativity of a diagram of the fourth type is satisfied in the level of chain complexes if the $\mathbb{A}$-diagrams (representing $\mathcal{H}_{i}$ ), and the complex structures on the surfaces are chosen correctly. Similarly and following the argument of [JT, Subsection 9.2], for suitable $\mathbb{A}$ diagrams representing the isotopy diagrams $\mathcal{H}_{i}$ and the correct choice of almost complex structures, the commutativity of the diagrams of the fifth type is satisfied in the level of chain complexes.

One may then copy the proof of [JT, Proposition 9.23] and show that if for the $\mathbb{A}$-diagram $(\Sigma, \boldsymbol{\alpha}, \boldsymbol{\beta}, \mathfrak{u}, \mathfrak{s})$ the map $d: \Sigma \rightarrow \Sigma$ is isotopic to identity, then $d_{*}=\Phi_{\beta \rightarrow \beta^{\prime}}^{\alpha \rightarrow \alpha^{\prime}}$, where $\alpha^{\prime}=d(\alpha)$ and $\beta^{\prime}=d(\beta)$. Thus, it follows from our definition of $\operatorname{HF}_{\mathbb{M}}(\mathcal{H})$ that the induced isomorphism $\operatorname{HF}^{\mathbb{M}}(d)$ is identity. This completes the proof of continuity.

Finally, let $\mathcal{H}_{1}, \mathcal{H}_{2}$ and $\mathcal{H}_{3}$ denote the isotopy diagrams corresponding to a handleswap. Choose A-diagrams $\left(\Sigma, \boldsymbol{\alpha}_{i}, \boldsymbol{\beta}_{i}, \mathfrak{u}, \mathfrak{s}_{i}\right)$ representing $\mathcal{H}_{i}, i=1,2,3$. The argument given for handleswap invariance in Subsection 9.3 of [JT] regards the region corresponding to the handleswap in the aforementioned Heegaard diagrams as a genus 2 subsurface $\Sigma^{0}$ of the connected sum of $\Sigma^{0}$ with another surface $\Sigma^{1}$, such that the Heegaard diagrams are identical on $\Sigma^{1}$, all markings in $\mathbf{z}$ lie on $\Sigma^{1}$, and two curves from each one of the attaching sets $\boldsymbol{\alpha}_{i}$ and $\boldsymbol{\beta}_{i}$ are on $\Sigma^{0}$. It is implied by [JT, Lemma 9.25 and Lemma 9.28] (which stay correct in our more general framework) that every triangle class which contributes to $\mathrm{HF}^{\mathbb{M}}\left(e_{1}\right)$ or $\mathrm{HF}^{\mathbb{M}}\left(e_{2}\right)$ may be decomposed as a disjoint union of a triangle class on $\Sigma^{1}$ which does not pass through the connected sum region and a small traingle class on $\Sigma^{0}$. The proof of Proposition 9.24 from [JT] thus goes through without difficulty, completing the proof of handleswap invariance.

Let $\mathfrak{h}$ denote an oriented path in $\mathcal{G}_{\mathcal{T}}$ from the isotopy diagram $\mathcal{H}$ to the isotopy diagram $\mathcal{H}^{\prime}$. Composing the isomorphisms corresponding to the edges in $\mathfrak{h}$ we obtain an isomorphism

$$
\operatorname{HF}^{\mathbb{M}}(\mathfrak{h}): \operatorname{HF}^{\mathbb{M}}(\mathcal{H}) \longrightarrow \operatorname{HF}^{\mathbb{M}}\left(\mathcal{H}^{\prime}\right) \text {. }
$$

If $\mathfrak{h}$ and $\mathfrak{h}^{\prime}$ are different oriented paths from $\mathcal{H}$ to $\mathcal{H}^{\prime},\left[\mathrm{JT}\right.$, Theorem 2.39], implies that $\operatorname{HF}^{\mathbb{M}}(\mathfrak{h})=$ $\mathrm{HF}^{\mathbb{M}}\left(\mathfrak{h}^{\prime}\right)$, since $\mathrm{HF}^{\mathbb{M}}$ is a strong Heegaard invariant. In other words, associated with the vertices $\mathcal{H}$ and $\mathcal{H}^{\prime}$ of $\mathcal{G}_{\mathcal{T}}$ there is a well-defined isomorphism

$$
\operatorname{HF}_{\mathcal{H} \rightarrow \mathcal{H}^{\prime}}^{\mathbb{M}}: \operatorname{HF}^{\mathbb{M}}(\mathcal{H}) \longrightarrow \operatorname{HF}^{\mathbb{M}}\left(\mathcal{H}^{\prime}\right) .
$$

It is clear that

One may thus define

$$
\mathrm{HF}_{\mathcal{H}^{\prime} \rightarrow \mathcal{H}^{\prime \prime}}^{\mathbb{M}} \circ \mathrm{HF}_{\mathcal{H} \rightarrow \mathcal{H}^{\prime}}^{\mathbb{M}}=\mathrm{HF}_{\mathcal{H} \rightarrow \mathcal{H}^{\prime \prime}}^{\mathbb{M}}
$$

$$
\operatorname{HF}^{\mathbb{M}}(\mathcal{T}):=\frac{\coprod_{\mathcal{H} \in\left|\mathcal{G}_{\mathcal{T}}\right|} \operatorname{HF}^{\mathbb{M}}(\mathcal{H})}{\sim}
$$

where $x$ is equivalent to $y$ if $y=\operatorname{HF}_{\mathcal{H} \rightarrow \mathcal{H}^{\prime}}^{\mathbb{M}}(x)$. Thus, associated with every $\mathbb{A}$-tangle $\mathcal{T}$ and $\mathbb{A}$-module $\mathbb{M}$ we obtain a well-defined $\mathbb{A}$-module $\operatorname{HF}^{\mathbb{M}}(\mathcal{T})$.

Let $d: \mathcal{T}=[M, T, \mathfrak{u}, \mathfrak{s}] \rightarrow \mathcal{T}^{\prime}=\left[M^{\prime}, T^{\prime}, \mathfrak{u}^{\prime}, \mathfrak{s}^{\prime}\right]$ be a diffeomorphism of $\mathbb{A}$-tangles. Pick an isotopy diagram $\mathcal{H}=(\Sigma, A, B, \mathfrak{u}, \mathfrak{s})$ for $\mathcal{T}$. The diffeomorphism $d$ takes $\Sigma$ to a surface $\Sigma^{\prime}$ in $M^{\prime}$ and the markings $\mathbf{z}$ to a set $\mathbf{z}^{\prime}$ of markings such that $\mathbf{z}^{\prime}=\Sigma^{\prime} \cap T^{\prime}$. Let $A^{\prime}=d(A)$ and $B^{\prime}=d(B)$. Furthermore, we obtain a $\operatorname{Spin}^{c}$ structure $\mathfrak{s}^{\prime}$ for $\left(\Sigma^{\prime}, A^{\prime}, B^{\prime}, \mathbf{z}^{\prime}\right)$ which corresponds to $\mathfrak{s}$. We may define $\mathfrak{u}^{\prime}: \mathbf{z}^{\prime} \rightarrow \mathbb{A}$ as $\mathfrak{u} \circ d^{-1}$. We thus obtain the isotopy diagram $\mathcal{H}^{\prime}=d(\mathcal{H})$ for $\mathcal{T}^{\prime}$. The isomorphism

$$
\operatorname{HF}^{\mathbb{M}}(d): \operatorname{HF}^{\mathbb{M}}(\mathcal{H}) \longrightarrow \operatorname{HF}^{\mathbb{M}}\left(\mathcal{H}^{\prime}\right)
$$


associated with the diffeomorphism $d$ from $H$ to $H^{\prime}$, induces a well-defined isomorphism

$$
\operatorname{HF}^{\mathbb{M}}(d): \operatorname{HF}^{\mathbb{M}}(\mathcal{T}) \longrightarrow \operatorname{HF}^{\mathbb{M}}\left(\mathcal{T}^{\prime}\right)
$$

The above considerations imply the following theorem.

Theorem 3.2. For every algebra $\mathbb{A}$ over $\mathbb{F}$ and every $\mathbb{A}$-module $\mathbb{M}$, assigning $\operatorname{HF}^{\mathbb{M}}(\mathcal{T})$ to the $\mathbb{A}$-tangle $\mathcal{T}$ in $\mathbb{A}$-Tang gives a functor

$$
\mathrm{HF}^{\mathbb{M}}: \mathbb{A} \text {-Tang } \longrightarrow \mathbb{A} \text {-Mod. }
$$

3.5. The action of $\Lambda^{*}\left(H_{1}(M, \mathbb{Z}) /\right.$ Tors $)$. As in the usual setup of the Heegaard Floer homology, there is a natural action of $\Lambda^{*}\left(H_{1}\left(M_{\mathcal{T}}, \mathbb{Z}\right) /\right.$ Tors $)$ on $\operatorname{HF}^{\mathbb{M}}(\mathcal{T})$ as follows. Let us assume that $H=$ $(\Sigma, \boldsymbol{\alpha}, \boldsymbol{\beta}, \mathfrak{u}: \mathbf{z} \rightarrow \mathbb{A}, \mathfrak{s})$ is an $\mathbb{A}$-diagram for the $\mathbb{A}$-tangle $\mathcal{T}=[M, T, \mathfrak{u}, \mathfrak{s}]$. First of all, as discussed in Subsection 2.4 of [OS04c], there is a homotopy long exact sequence

$$
0 \longrightarrow \mathbb{Z} \longrightarrow \pi_{1}\left(\Omega\left(\mathbb{T}_{\alpha}, \mathbb{T}_{\beta}\right)\right) \longrightarrow \pi_{1}\left(\mathbb{T}_{\alpha} \times \mathbb{T}_{\beta}\right) \longrightarrow \pi_{1}\left(\operatorname{Sym}^{g}(\Sigma)\right)
$$

Here $\Omega\left(\mathbb{T}_{\alpha}, \mathbb{T}_{\beta}\right)$ denotes the space of paths in $\operatorname{Sym}^{g}(\Sigma)$ joining $\mathbb{T}_{\alpha}$ to $\mathbb{T}_{\beta}$. Under the identification of $\pi_{1}\left(\operatorname{Sym}^{g}(\Sigma)\right)$ with $H^{1}(\Sigma, \mathbb{Z}), \pi_{1}\left(\mathbb{T}_{\alpha}\right)$ and $\pi_{1}\left(\mathbb{T}_{\beta}\right)$ correspond to $H^{1}(C(\boldsymbol{\alpha}), \mathbb{Z})$ and $H^{1}(C(\boldsymbol{\beta}), \mathbb{Z})$, respectively. After comparing the above exact sequence with the cohomology long exact sequence for the decomposition $M=C(\boldsymbol{\alpha}) \cup_{\Sigma} C(\boldsymbol{\beta})$, we obtain the short exact sequence

$$
0 \longrightarrow \mathbb{Z} \longrightarrow \pi_{1}\left(\Omega\left(\mathbb{T}_{\alpha}, \mathbb{T}_{\beta}\right)\right) \longrightarrow H^{1}(M, \mathbb{Z}) \longrightarrow 0 .
$$

Applying $\operatorname{Hom}(-, \mathbb{Z})$ to the above short exact sequence we obtain

$$
0 \longrightarrow H_{1}(M, \mathbb{Z}) / \text { Tors } \longrightarrow H^{1}\left(\Omega\left(\mathbb{T}_{\alpha}, \mathbb{T}_{\beta}\right), \mathbb{Z}\right) \longrightarrow \mathbb{Z} .
$$

Every element $\zeta \in H_{1}(M, \mathbb{Z}) /$ Tors may thus be realized as an element of $H^{1}\left(\Omega\left(\mathbb{T}_{\alpha}, \mathbb{T}_{\beta}\right), \mathbb{Z}\right)$, which is represented by a 1 -cocycle $Z(\zeta) \in Z^{1}\left(\Omega\left(\mathbb{T}_{\alpha}, \mathbb{T}_{\beta}\right), \mathbb{Z}\right)$ in the space of paths connecting $\mathbb{T}_{\alpha} \cap \mathbb{T}_{\beta}$. If $\phi \in \pi_{2}(\mathbf{x}, \mathbf{y})$ is the homotopy class of a Whitney disk, it may be viewed as an arc in $\Omega\left(\mathbb{T}_{\alpha}, \mathbb{T}_{\beta}\right)$ which connects the constant path at $\mathbf{x}$ to the constant path at $\mathbf{y}$. The evaluation of $Z(\zeta)$ over this path gives a value $n_{Z(\zeta)}(\phi)$. Correspondingly, we may define a map

$$
\begin{aligned}
& A_{Z(\zeta)}: \mathrm{CF}(\Sigma, \boldsymbol{\alpha}, \boldsymbol{\beta}, \mathfrak{u}, \mathfrak{s}) \rightarrow \mathrm{CF}(\Sigma, \boldsymbol{\alpha}, \boldsymbol{\beta}, \mathfrak{u}, \mathfrak{s}) \\
& A_{Z(\zeta)}(\mathbf{x}):=\sum_{\substack{\mathbf{y} \in \mathbb{T}_{\alpha} \cap \mathbb{T}_{\beta} \\
\mathfrak{s}(\mathbf{y})=\mathfrak{s}}} \sum_{\phi \in \pi_{2}^{1}(\mathbf{x}, \mathbf{y})} n_{Z(\zeta)}(\phi) \mathfrak{m}(\phi) \mathfrak{u}(\phi) \mathbf{y} .
\end{aligned}
$$

The proof of [OS04c, Lemma 4.18] implies that $A_{Z(\zeta)}$ is a chain map and the proof of [OS04c, Lemma 4.19] implies that if $Z(\zeta)$ is a coboundary then $A_{Z(\zeta)}$ is chain homotopic to zero. The map induced by $A_{Z(\zeta)}$ on homology is thus independent of the choice of the cocycle $Z(\zeta)$, and may thus be represented by

$$
A_{\zeta}: \operatorname{HF}^{\mathbb{M}}(\Sigma, \boldsymbol{\alpha}, \boldsymbol{\beta}, \mathfrak{u}, \mathfrak{s}) \rightarrow \operatorname{HF}^{\mathbb{M}}(\Sigma, \boldsymbol{\alpha}, \boldsymbol{\beta}, \mathfrak{u}, \mathfrak{s}) .
$$

The proof of [OS04c, Proposition 4.17] may then be copied to show that $A_{\zeta} \circ A_{\zeta}=0$. Consequently, we obtain an action of the exterior algebra $\Lambda^{*}\left(H_{1}(M, \mathbb{Z}) /\right.$ Tors $)$ on $\operatorname{HF}^{\mathbb{M}}(\Sigma, \boldsymbol{\alpha}, \boldsymbol{\beta}, \mathfrak{u}, \mathfrak{s})$.

We may then follow the steps toward weak and strong Heegaard invariance of the functor $\mathrm{HF}^{\mathbb{M}}$, and observe that all the isomorphisms which correspond to the edges of the graph $\mathcal{G}_{\mathcal{T}}$ preserve the action of $\Lambda^{*}\left(H_{1}(M, \mathbb{Z}) /\right.$ Tors $)$ constructed above. This observation implies the following proposition.

Proposition 3.3. For every $\mathbb{A}$-tangle $\mathcal{T}$ and every $\mathbb{A}$-module $\mathbb{M}$, there is a natural action of $\Lambda^{*}\left(H_{1}\left(M_{\mathcal{T}}, \mathbb{Z}\right) /\right.$ Tors $)$ on $\operatorname{HF}^{\mathbb{M}}(\mathcal{T})$. 


\section{Parametrized Cerf decomposition}

4.1. Parametrized elementary cobordism. Let $(M, T)$ be a balanced tangle. For $k=0,1,2$, a framed $k$-sphere $\mathbb{S}$ in $(M, T)$ is an embedding of $S^{k} \times D^{3-k}$ in $M-T$. In other word, it is an embedded $k$-sphere $a(\mathbb{S})=S^{k} \times\{0\}$, called attaching sphere, in $M-T$ together with a trivialization $\nu$ of its normal bundle. We will denote by

$$
\mathcal{W}(\mathbb{S})=(W(\mathbb{S}), F(\mathbb{S}))
$$

the cobordism obtained by attaching a $k$-handle to $M \times[0,1]$ along $\mathbb{S} \times\{1\}$ to construct $W(\mathbb{S})$ and setting $F(\mathbb{S})=T \times[0,1] \subset W(\mathbb{S})$. Thus $\mathcal{W}(\mathbb{S})$ is a cobordism from $(M, T)$ to $(M(\mathbb{S}), T)$, where $M(\mathbb{S})$ is obtained by surgery on $M$ along $\mathbb{S}$.

Similarly, a framed arc $\mathbb{I}$ in $(M, T)$ is an embedding of $D^{2} \times D^{1}$ in $M$ such that

$$
\mathbb{I}^{-1}(T)=(\{x=0\} \times\{-1\}) \coprod(\{x=0\} \times\{1\}),
$$

where $(x, y)$ denotes the standard coordinate system on $D^{2}$ (as a subset of $\mathbb{R}^{2}$ ). Moreover, $\mathbb{I}$ is called orientation preserving if the restriction of $\mathbb{I}$ to

$$
\{x=0\} \times\{-1\} \coprod\{x=0\} \times\{1\} \subset \partial(\{x=0\} \times[-1,1]),
$$

which is equipped with the boundary orientation, is orientation preserving as a map to $T$. We think of $\mathbb{I}$ as an embedded arc $a(\mathbb{I})=\{0\} \times D^{1}$, called attaching arc, connecting the two points $\partial a(\mathbb{I}) \cap T$, together with a trivialization $\nu$ of its normal bundle in $M$ which is compatible at the end points, with the trivialization induced from the orientation of $T$. Abusing the notation, we denote $\mathbb{I}\left(D^{2} \times D^{1}\right)$ by $\mathbb{I}$. Associated with a framed arc $\mathbb{I}$, let $T(\mathbb{I}) \subset M$ be the properly embedded 1-manifold obtained by doing band surgery on $T$ along $\mathbb{I}$ i.e.

$$
T(\mathbb{I})=(T-\mathbb{I} \cap T)) \bigcup \mathbb{I}((\{x=-1\} \cup\{x=1\}) \times[-1,1]) .
$$

Note that if $\mathbb{I}$ is orientation preserving, then the orientation on $T$ induces an orientation on $T(\mathbb{I})$. Moreover, for every set $\mathbb{I}=\left\{\mathbb{I}_{1}, \ldots, \mathbb{I}_{n}\right\}$ of framed arcs in $(M, T)$, denote the properly embedded 1-manifold constructed by doing surgery on $T$ along the framed $\operatorname{arcs} \mathbb{I}_{1}, \ldots, \mathbb{I}_{n}$ by $T(\mathbb{I})$. Thus, if $\mathbb{I}$ is a single framed arc, $T(\mathbb{I})$ and $T(\{\mathbb{I}\})$ are the same object.

Definition 4.1. A set $\mathbb{I}=\left\{\mathbb{I}_{1}, \ldots, \mathbb{I}_{n}\right\}$ of framed arcs in $(M, T)$ is called acceptable if for any $i, \mathbb{I}_{i}$ is orientation preserving and $(M, T(\mathbb{I}))$ is a tangle.

Let $\mathbb{I}=\left\{\mathbb{I}_{1}, \ldots, \mathbb{I}_{n}\right\}$ be an acceptable set of framed arcs in $(M, T)$. Corresponding to $\mathbb{I}$, we construct a cobordism $\mathcal{W}(\mathbb{I})=(W(\mathbb{I}), F(\mathbb{I}))$ from $(M, T)$ to $(M, T(\mathbb{I}))$ by attaching a standard pair to $(M \times[0,1], T \times[0,1])$ along $\mathbb{I} \subset M \times\{1\}$ as follows. The standard saddle is the pair $(H, B)$, which is identified in $\mathbb{R}^{4}$ via

$$
\begin{aligned}
& H:=D^{2} \times D^{1} \times D^{1}=\left\{(x, y, z, t) \in \mathbb{R}^{4} \mid \begin{array}{c}
x^{2}+y^{2} \leq 1 \\
z, t \in[-1,1]
\end{array}\right\} \text { and } \\
& B=\left\{(x, y, z, t) \in D^{2} \times D^{1} \times D^{1} \mid \begin{array}{c}
(t+1) y^{2}+(t-1) z^{2}=2 t \\
x=0
\end{array}\right\}
\end{aligned}
$$

For $i \in\{-1,1\}$, denote $\partial_{i}(H, B):=\left(D^{2} \times D^{1} \times\{i\}, B \cap\left(D^{2} \times D^{1} \times\{i\}\right)\right)$. See Figure 1 for a picture of the projection of the standard pair over the 3 -dimensional box $\{x=0\} \times D^{1} \times D^{1}$.

Let $\mathcal{W}(\mathbb{I})=(W(\mathbb{I}), F(\mathbb{I}))$ denote the cobordism

$$
\frac{(M \times[0,1], T \times[0,1]) \bigsqcup\left(\coprod_{i=1}^{n}\left(H_{i}, B_{i}\right)\right)}{\left\{\left(\mathbb{I}_{i} \times\{1\},\left(T \cap \mathbb{I}_{i}\right) \times\{1\}\right) \sim \partial_{-1}\left(H_{i}, B_{i}\right) \mid \text { for any } 1 \leq i \leq n\right\}}
$$

after smoothing the corresponding corners. Here $\left(H_{i}, B_{i}\right)$ are copies of the standard pair $(H, B)$ for $i=1, \ldots, n$. Note that $W(\mathbb{I}) \simeq M \times[0,1]$. 


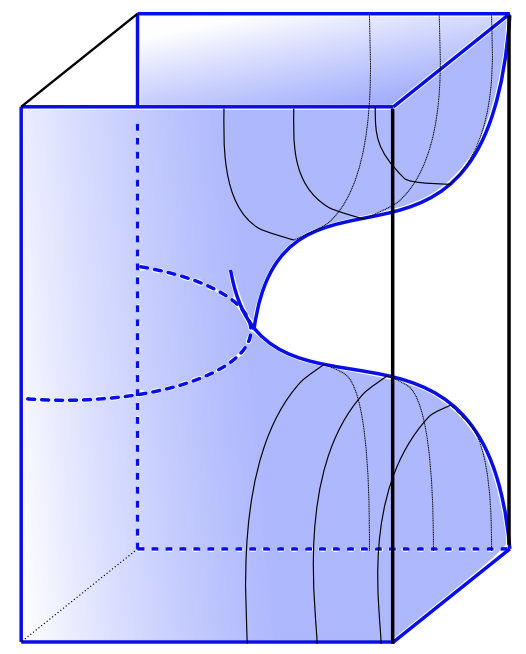

Figure 1. The standard pair, which is pictured in the cube $\{x=0\}=[-1,1]^{3}$.

Definition 4.2. A stable cobordism $\mathcal{W}=(W, F)$ from the tangle $(M, T)$ to the tangle $\left(M^{\prime}, T^{\prime}\right)$ is called elementary, if for a framed sphere $\mathbb{S}$ or an acceptable set of framed $\operatorname{arcs} \mathbb{I}$ in $(M, T), \mathcal{W}$ is diffeomorphic to the corresponding cobordism $\mathcal{W}(\mathbb{S})$ or $\mathcal{W}(\mathbb{I})$.

Moreover, a parametrized elementary cobordism is an elementary cobordism $\mathcal{W}$ as above, accompanied with one of the following:

(1) A framed sphere $\mathbb{S} \subset M$, together with the isotopy class of a diffeomorphism

$$
d:(M(\mathbb{S}), T) \rightarrow\left(M^{\prime}, T^{\prime}\right)
$$

so that for a diffeomorphism $D: \mathcal{W}(\mathbb{S}) \rightarrow \mathcal{W}$ with $\left.D\right|_{(M, T)}=\mathrm{Id}$, we have $d=\left.D\right|_{(M(\mathbb{S}), T)}$.

(2) A framed arc $\mathbb{I} \subset M$, together with the isotopy class of a diffeomorphism

$$
d:(M, T(\mathbb{I})) \rightarrow\left(M^{\prime}, T^{\prime}\right)
$$

so that for a diffeomorphism $D: \mathcal{W}(\mathbb{I}) \rightarrow \mathcal{W}$ with $\left.D\right|_{(M, T)}=\operatorname{Id}$, we have $d=\left.D\right|_{(M, T(\mathbb{I}))}$.

Note that in the above definition we might also have $\mathbb{S}=\emptyset$.

Definition 4.3. A parametrized Cerf decomposition of a stable cobordism $\mathcal{W}=(W, F)$ from a tangle $(M, T)$ to a tangle $\left(M^{\prime}, T^{\prime}\right)$ is a decomposition

$$
\mathcal{W}=\mathcal{W}_{1} \cup_{\left(M_{1}, T_{1}\right)} \cdots \cup_{\left(M_{m-1}, T_{m-1}\right)} \mathcal{W}_{m}
$$

where $\mathcal{W}_{i}=\left(W_{i}, F_{i}\right)$ is a parametrized elementary cobordism from the tangle $\left(M_{i-1}, T_{i-1}\right)$ to the tangle $\left(M_{i}, T_{i}\right),\left(M_{0}, T_{0}\right)=(M, T)$ and $\left(M_{m}, T_{m}\right)=\left(M^{\prime}, T^{\prime}\right)$. For every $i=1, \ldots, m$, depending on the type of $\mathcal{W}_{i}$, we let $\left(\mathbb{S}_{i}, d_{i}\right)$ or $\left(\mathbb{I}_{i}, d_{i}\right)$ denote its parametrization.

4.2. Parametrized Morse data. Let $\mathcal{W}=(W, F)$ be a cobordism from $(M, T)$ to $\left(M^{\prime}, T^{\prime}\right)$. A function $G: W \rightarrow[a, b]$ is called a Morse function on $\mathcal{W}$, if $G$ has no critical points in a neighborhood of $\partial W \cup F$, both $G$ and $g=\left.G\right|_{F}$ are Morse (on $W$ and $F$, respectively) and

$$
G^{-1}(a)=M, \quad G^{-1}(b)=M^{\prime} \quad \text { and }\left.\quad G\right|_{\partial_{h} W}=\pi_{2},
$$

where $\pi_{2}$ is the projection over the second factor under an identification

$$
\left(\partial_{h} W, \partial_{h} F\right)=(\partial M, \partial T) \times[a, b] .
$$

The set of critical points of $G$ on $\mathcal{W}$, $\operatorname{Crit}_{\mathcal{W}}(G)$, is defined as

$$
\operatorname{Crit}_{\mathcal{W}}(G):=\operatorname{Crit}_{W}(G) \cup \operatorname{Crit}(g)
$$

where $\operatorname{Crit}_{W}(G)$ and $\operatorname{Crit}(g)$ are the sets of critical points of $G$ on $W$ and $g=\left.G\right|_{F}$ on $F$, respectively. A Morse function $G$ on $\mathcal{W}$ is called proper if it has distinct values at its critical points on $\mathcal{W} . G$ is called indefinite if both $G$ and $g$, as Morse functions on $W$ and $F$ respectively, are 
indefinite i.e. have no critical points with the minimal and maximal index. Therefore, if $G$ is indefinite on $\mathcal{W}, g$ has only critical points of index 1, while the critical points of $G$ are of indices 1,2 or 3.

Definition 4.4. Let $G: W \rightarrow[a, b]$ be a Morse function on the cobordism $\mathcal{W}=(W, F)$. A vector field $\xi$ on $W$ is called an embedded gradient-like vector field for $G$ if it satisfies the following conditions:

(1) For every $p \in W$ which is not in $\operatorname{Crit}_{\mathcal{W}}(G), d G_{p}\left(\xi_{p}\right)>0$.

(2) The vector field $\xi$ is tangent to both $F$ and $\partial_{h} W$.

(3) For any critical point $p \in \operatorname{Crit}_{W}(G)$ there is an open neighborhood $U \subset W$ of $p$ together with a positively oriented local coordinate system $\left(x_{1}, x_{2}, x_{3}, x_{4}\right)$ centered at $p$ such that

$G\left(x_{1}, x_{2}, x_{3}, x_{4}\right)=G(p) \pm x_{1}^{2} \pm x_{2}^{2} \pm x_{3}^{2} \pm x_{4}^{2} \quad$ and $\quad \xi\left(x_{1}, x_{2}, x_{3}, x_{4}\right)=\left( \pm x_{1}, \pm x_{2}, \pm x_{3}, \pm x_{4}\right)$

(4) There is an open neighborhood $U \subset W$ of every critical point $p$ of $g$ and a positively oriented local coordinate system $\left(x_{1}, x_{2}, y_{1}, y_{2}\right)$ centered at $p$, such that

$$
\begin{aligned}
& U \cap F=\left\{\left(x_{1}, x_{2}, y_{1}, y_{2}\right) \in U \mid y_{1}=y_{2}=0\right\} \\
& G\left(x_{1}, x_{2}, y_{1}, y_{2}\right)=G(p) \pm x_{1}^{2} \pm x_{2}^{2}+y_{1} \quad \text { and } \quad \xi=\left( \pm x_{1}, \pm x_{2}, y_{1}^{2}+y_{2}^{2}, 0\right) .
\end{aligned}
$$

Definition 4.5. A Morse datum for $\mathcal{W}$ is a triple $\mathfrak{M}=(G, \underline{b}, \xi)$, where

$$
\underline{b}=\left(a=b_{0}<\cdots<b_{m}=b\right) \in \mathbb{R}^{m+1},
$$

is an ordered $(m+1)$-tuple of regular values for the proper Morse function $G: W \rightarrow[a, b]$ on $\mathcal{W}$, and $\xi$ is an embedded gradient like vector field for $G$. Over each interval $\left(b_{i}, b_{i+1}\right), G$ has at most one critical point in $W$, and if it has a critical point in $G^{-1}\left(b_{i}, b_{i+1}\right)$ then $g$ has no critical point over this interval.

The Morse datum $(G, \underline{b}, \xi)$ is called good if $g$ is indefinite and $g^{-1}\left(b_{i}\right)$ is a union of arcs connecting $\partial_{h}^{-} W$ to $\partial_{h}^{+} W$ for $i=0, \ldots, m$.

Any good Morse datum $\mathfrak{M}=(G, \underline{b}, \xi)$ induces a parametrized Cerf decomposition $\mathfrak{C}(G, \underline{b}, \xi)$ of $\mathcal{W}$ by taking $W_{i}=G^{-1}\left[b_{i-1}, b_{i}\right], F_{i}=g^{-1}\left[b_{i-1}, b_{i}\right]$ and $\left(M_{i}, T_{i}\right)=\left(G^{-1}\left(b_{i}\right), g^{-1}\left(b_{i}\right)\right)$. If $G$ has a critical point $p \in G^{-1}\left[b_{i-1}, b_{i}\right]$ of index $k_{i}$, the descending flow of $\xi$ maps $p$ to a $k_{i}-1$ dimensional embedded sphere $S_{i} \subset G^{-1}\left(b_{i-1}\right)$, which is disjoint from $T_{i-1}$. Moreover, using a positive local coordinate system in the neighborhood of $p$ such that $G$ and $\xi$ have the standard structure, we obtain a framing for $S_{i}$. As in Remark 2.12 of [Juh], if $k_{i}=0,4$, the framed sphere $\mathbb{S}_{i}$ does not depend on the choice of the local coordinates and is uniquely determined up to isotopy. Otherwise, for $k \neq 0,4$, depending on the positive local coordinate system, we obtain two non-isotopic embedded framed sphere $\mathbb{S}_{i}$ and $\overline{\mathbb{S}}_{i}$. Note that for any framed sphere $\mathbb{S}: S^{k-1} \times D^{4-k} \rightarrow M-T$ in a tangle $(M, T), \overline{\mathbb{S}}$ is defined by

$$
\overline{\mathbb{S}}(x, y)=\mathbb{S}\left(r_{k}(x), r_{4-k}(y)\right)
$$

for $x \in S^{k-1} \subset \mathbb{R}^{k}, y \in D^{4-k} \subset \mathbb{R}^{4-k}$, where

$$
r_{k}\left(x_{1}, x_{2}, \ldots, x_{k}\right)=\left(-x_{1}, x_{2}, \ldots, x_{k}\right) .
$$

Along with the framed sphere $\mathbb{S}_{i}$, we obtain a diffeomorphism

$$
d_{i}:\left(M_{i-1}\left(\mathbb{S}_{i}\right), T_{i-1}\right) \longrightarrow\left(M_{i}, T_{i}\right)
$$

which is given using the flow of the vector field $\xi$ over $M_{i-1} \backslash \operatorname{nd}\left(\mathbb{S}_{i}\right)$, the complement of the framed sphere $\mathbb{S}_{i}$. On the other hand, if $g$ has index one critical points $p_{1}, \ldots, p_{n_{i}} \in G^{-1}\left[b_{i-1}, b_{i}\right] \cap F$, the descending manifolds of $p_{1}, \ldots, p_{n_{i}}$ under the flow of $\xi$ determines a set of orientation preserving framed $\operatorname{arcs} \mathbb{I}_{i}=\left\{\mathbb{I}_{1}^{i}, \ldots, \mathbb{I}_{n_{i}}^{i}\right\}$ with end points on $T_{i-1}=g^{-1}\left(b_{i-1}\right)$. Since $g^{-1}\left(b_{i}\right)$ is a union of arcs connecting $\partial^{-} M_{i}$ to $\partial^{+} M_{i}$, the set $\mathbb{I}_{i}$ is acceptable. The flow of $\xi$ gives a diffeomorphism

$$
d_{i}:\left(M_{i-1}, T_{i-1}\left(\mathbb{I}_{i}\right)\right) \longrightarrow\left(M_{i}, T_{i}\right) .
$$


Furthermore, if $G$ has no critical points in $\mathcal{W}_{i}$, we set $\mathbb{S}_{i}=\emptyset$ and the flow of $\xi$ defines a diffeomorphism $d_{i}$ from $\left(M_{i-1}, T_{i-1}\right)$ to $\left(M_{i}, T_{i}\right)$.

Therefore, any good Morse datum $\mathfrak{M}=(G, \underline{b}, \xi)$ defines a parametrized Cerf decomposition for $\mathcal{W}$ denoted by $\mathfrak{C}(\mathfrak{M})$, well-defined up to replacing some of the framed spheres $\mathbb{S}$ by the corresponding framed spheres $\overline{\mathbb{S}}$. Because of this ambiguity, we say two good Morse data $\mathfrak{M}$ and $\mathfrak{M}^{\prime}$ induce the same parametrized Cerf decompositions for $\mathcal{W}$ if and only if the corresponding attaching spheres and attaching arcs coincide, while they are isotopic as framed spheres or framed arcs after replacing some framed spheres $\mathbb{S}$ in $\mathfrak{C}(\mathfrak{M})$ with $\overline{\mathbb{S}}$.

Lemma 4.1. Let $\mathcal{W}$ be a stable cobordism. Then every parametrized Cerf decomposition for $\mathcal{W}$ is induced by a good Morse datum.

Proof. Suppose that $\mathcal{W}$ is an elementary cobordism from $(M, T)$ to $\left(M^{\prime}, T^{\prime}\right)$.

Case 1. $\mathcal{W}$ is parametrized by a framed sphere $\mathbb{S}$ in $(M, T)$ and a diffeomorphism $d:(M(\mathbb{S}), T) \rightarrow$ $\left(M^{\prime}, T^{\prime}\right)$. It follows from the proof of [Juh, Lemma 2.14], that one can define a Morse function $G^{\prime}: W(\mathbb{S}) \rightarrow[0,1]$ together with an embedded gradient-like vector field $\xi^{\prime}$ on $\mathcal{W}(\mathbb{S})=(W(\mathbb{S}), F(\mathbb{S}))$ such that $G^{\prime}$ has a single critical point, while $g^{\prime}=\left.G^{\prime}\right|_{F(\mathbb{S})}$ has no critical points. Furthermore, the diffeomorphism induced by $\xi^{\prime}$ on $(M(\mathbb{S}), T)$ is $\operatorname{Id}_{M(\mathbb{S})}$. For instance, if $\mathbb{S}=\emptyset$, then for the cobordism $\mathcal{W}(\mathbb{S})=(M, T) \times[0,1]$ we let $G^{\prime}=\pi_{2}$ and $\xi^{\prime}=\partial_{t}$. Then, $G=G^{\prime} \circ D^{-1}$ and $\xi=D_{\star} \xi^{\prime}$ is the corresponding good Morse datum, where $D: \mathcal{W}(\mathbb{S}) \rightarrow \mathcal{W}$ is a diffeomorphism such that $\left.D\right|_{(M, T)}=\operatorname{Id}$ and $\left.D\right|_{(M(\mathbb{S}), T)}=d$.

Case 2. $\mathcal{W}$ is parametrized by an acceptable set of framed arcs $\mathbb{I}=\left\{\mathbb{I}_{1}, \ldots, \mathbb{I}_{n}\right\}$, and a diffeomorphism $d:(M, T(\mathbb{I})) \rightarrow\left(M^{\prime}, T^{\prime}\right)$. For any $i$ consider a small neighborhood $N_{i}$ of $\mathbb{I}_{i}$ in $(M, T)$. Let

$$
\left(W_{i}, F_{i}\right):=\frac{\left(N_{i}, N_{i} \cap T\right) \times[0,1] \coprod\left(H_{i}, B_{i}\right)}{\left(\mathbb{I}_{i} \times\{1\},\left(T \cap \mathbb{I}_{i}\right) \times\{1\}\right) \sim \partial_{-1}\left(H_{i}, B_{i}\right)} \subset \mathcal{W}(\mathbb{I}) .
$$

Here $\left(H_{i}, B_{i}\right)$ denotes a copy of the standard saddle $(H, B)$ for $i=1, \ldots, n$. Let $N$ be a small neighborhood of $D^{2} \times D^{1}$ in $\mathbb{R}^{3}$ and $\widetilde{B}$ denote the corresponding saddle in $N \times D^{1}$. After smoothing the corners obtained from attaching the saddles, $\left(W_{i}, F_{i}\right)$ is diffeomorphic with $\left(N \times D^{1}, \widetilde{B}\right)$. Choose a diffeomorphism

$$
\phi_{i}:\left(W_{i}, F_{i}\right) \longrightarrow\left(N \times D^{1}, \widetilde{B}\right)
$$

such that

$$
\begin{aligned}
& \phi_{i}\left(\partial_{1}\left(H_{i}, B_{i}\right)\right)=\left(D^{2} \times D^{1} \times\{1\}, \widetilde{B} \cap\left(D^{2} \times D^{1} \times\{1\}\right)\right) \text { and } \\
& \phi_{i}\left(N_{i} \times\{0\},\left(N_{i} \cap T\right) \times\{0\}\right)=(N \times\{-1\},(\widetilde{B} \cap N) \times\{-1\}) .
\end{aligned}
$$

Moreover, for a sufficiently small $\nu \in \mathbb{R}^{+}$and every $t \in(0,1)$, if $(x, y, z) \in N$ belongs to the $\nu$-neighborhood of $\partial N$, then

$$
\phi^{-1}(x, y, z, 2 t-1) \in N_{i} \times\{t\}
$$

We define $G_{i}^{\prime}$ by

$$
G_{i}^{\prime}(y):= \begin{cases}\pi_{2}(y) & \text { if } y \in M \times[0,1]-\amalg_{i=1}^{n} W_{i} \\ \frac{\pi_{2}\left(\phi_{i}(y)\right)+1}{2} & \text { if } y \in W_{i}\end{cases}
$$

This is a smooth function, by construction. We define the embedded gradient-like vector field $\xi^{\prime}$ on $\mathcal{W}(\mathbb{I})$ by pulling back the vector field $2 \partial_{t}$ on $N \times D^{1}$ using $\phi_{i}$ and extending it to the rest of $M \times[0,1]$ using $\partial_{t}$. It is now straightforward to check that the Morse function $G^{\prime}$ together with the embedded gradient-like vector field $\xi^{\prime}$ induces the identity diffeomorphism on $(M, T(\mathbb{I}))$. Hence, considering a diffeomorphism $D: \mathcal{W}(\mathbb{I}) \rightarrow \mathcal{W}$ for which $\left.D\right|_{(M, T)}=\operatorname{Id}$ and $\left.D\right|_{(M, T(\mathbb{I}))}=d$, the Morse function $G=G^{\prime} \circ D^{-1}$ and the gradient-like vector field $\xi=D_{\star} \xi^{\prime}$ is the corresponding Morse datum. 
If $\mathcal{W}$ is not an elementary cobordism, consider a parametrized Cerf decomposition

$$
\mathcal{W}=\mathcal{W}_{1} \cup_{\left(M_{1}, T_{1}\right)} \cdots \cup_{\left(M_{m-1}, T_{m-1}\right)} \mathcal{W}_{m}
$$

for $\mathcal{W}$. For each $\mathcal{W}_{i}$ denote the corresponding Morse datum constructed as above by $\left(G_{i}, \xi_{i}\right)$. Fix an $(m+1)$-tuple $\underline{b}=\left(a=b_{0}<b_{1}<\cdots<b_{m}=b\right)$ of real numbers, and let $a_{i}:[0,1] \rightarrow\left[b_{i-1}, b_{i}\right]$ be the diffeomorphism $a_{i}(t)=(1-t) b_{i-1}+t b_{i}$. Then, we can modify the Morse functions $a_{i} \circ G_{i}$ and the gradient-like vector fields $\xi_{i}$, on a collar neighborhood of $\left(M_{i}, T_{i}\right)$ such that they fit together to give a Morse function $G$ and an embedded gradient-like vector field $\xi$ on $\mathcal{W}$.

Lemma 4.2. Suppose $\mathfrak{M}=(G, \underline{b}, \xi)$ and $\mathfrak{M}^{\prime}=\left(G^{\prime}, \underline{b}^{\prime}, \xi^{\prime}\right)$ are two good Morse data for a stable cobordism $\mathcal{W}=(W, F)$ that induce the same parametrized Cerf decompositions. There exist diffeomorphisms $D: \mathcal{W} \rightarrow \mathcal{W}$ and $\phi: \mathbb{R} \rightarrow \mathbb{R}$ satisfying the followings:

(1) $\underline{b}^{\prime}=\phi(\underline{b})$

(2) $G^{\prime}=\phi \circ G \circ D^{-1}$

(3) for some $h \in C^{\infty}\left(W, \mathbb{R}^{+}\right)$we have $D_{*} \xi=h . \xi^{\prime}$

(4) $\left.D\right|_{M}=\operatorname{Id}_{M}$ and $\left.D\right|_{M^{\prime}}=\operatorname{Id}_{M^{\prime}}$.

Proof. See proof of [Juh, Lemma 2.14].

Proposition 4.3. For every stable cobordism $\mathcal{W}$ there exists a good Morse datum $\mathfrak{M}=(G, \underline{b}, \xi)$, and therefore a parametrized Cerf decomposition.

Proof. Let $\mathcal{W}=(W, F)$ be a stable cobordism from $(M, T)$ to $\left(M^{\prime}, T^{\prime}\right)$ and let $g: F \rightarrow[0,1]$ be a Morse function satisfying

$$
\left.g\right|_{T} \equiv 0,\left.\quad g\right|_{T^{\prime}} \equiv 1, \quad \text { and }\left.\quad g\right|_{F \cap \partial_{h} W}=\pi_{2} .
$$

Since every connected component of $F$ has non-empty intersection with either of $T$ and $T^{\prime}$, we may assume that $g$ has no minimal or maximal index critical point i.e. all critical points of $g$ have index 1. Further, we assume that it has the same value over all of its critical points. We extend $g$ to a tubular neighborhood of $F$ such that it has no critical points and then extend it to a Morse function $G: W \rightarrow[0,1]$. By a small perturbation in a neighborhood of the critical points of $G$, we may assume that $G$ has distinct critical values and these values are distinct from the critical value of $g$. Finally, by a small perturbation in a neighborhood of the critical points of $g$ we can transform $G$ into a proper Morse function on $\mathcal{W}$, such that for every $p, q \in \operatorname{Crit}(g)$ with $G(p)<G(q), G$ has no critical point on $W$ above the interval $(G(p), G(q))$. It is straightforward to show that there is an embedded gradient-like vector field for every Morse function on $\mathcal{W}$. Let $\xi$ be such a gradient-like vector field for $G$. Choose the ordered set

$$
\underline{b}=\left\{0=b_{0}<b_{1}<\cdots<b_{m}=1\right\} \subset[0,1]
$$

of regular values for $G$ such that for every $i=1, \ldots, m$, $\operatorname{Crit}_{W}(G)$ has at most one element in $G^{-1}\left[b_{i-1}, b_{i}\right]$. Furthermore, for exactly one $1 \leq j \leq m$ all critical values of $g$ lie in $\left(b_{j-1}, b_{j}\right)$, while $\operatorname{Crit}_{W}(G)$ does not intersect $G^{-1}\left[b_{j-1}, b_{j}\right]$. The triple $(G, \underline{b}, \xi)$ is a good Morse datum for $\mathcal{W}$ and it thus gives a parametrized Cerf decomposition of $\mathcal{W}$.

4.3. Cerf moves. In this section, we describe Cerf moves on Morse data and discuss how corresponding parametrized Cerf decompositions change under these moves. For this purpose, fix a cobordism $\mathcal{W}=(W, F)$ from $(M, T)$ to $\left(M^{\prime}, T^{\prime}\right)$. Let $\mathfrak{M}=(G, \underline{b}, \xi)$ and $\mathfrak{M}^{\prime}=\left(G^{\prime}, \underline{b}^{\prime}, \xi^{\prime}\right)$ be parametrized Morse data for $\mathcal{W}$ so that $G, G^{\prime}: W \rightarrow[a, b]$ are proper Morse functions on $\mathcal{W}$.

Critical point cancellation/creation. The Morse data $\mathfrak{M}$ and $\mathfrak{M}^{\prime}$ are related by a critical point cancellation if 
(1) $G$ and $G^{\prime}$ are related by a critical point cancellation i.e. there exist a family of smooth functions $\left\{G_{t}: W \rightarrow[a, b] \mid t \in[-1,1]\right\}$ such that $G_{-1}=G, G_{1}=G^{\prime}$ and:

- For $t \in[-1,1]-\{0\}, G_{t}$ is a proper Morse function on $\mathcal{W}$ and $G_{0}$ has a death singularity at some point $p_{\circ} \in W$,

- The family is an elementary death ('chemin élémentaire de mort' in the sense of Cerf Cer70, Section 2.3, p.71]) with support in a neighborhood $U$ of $p_{\circ}$. More precisely, $G_{t}$ does not depend on $t$ outside $U$ and for $t \in(0,1]$ it has no critical points in $U$. Furthermore, there exist local coordinates $\left(x_{1}, \ldots, x_{4}\right)$ around $p_{\circ}$, such that for $t \in[-1,1]$

$$
G_{t}\left(x_{1}, \ldots, x_{4}\right)=G_{0}\left(p_{\circ}\right)+x_{1}^{3}+t x_{1}-x_{2}^{2} \cdots-x_{k}^{2}+x_{k+1}^{2}+\cdots+x_{4}^{2}
$$

(2) Assume $\underline{b}=\left(a=b_{0}<b_{1}<\cdots<b_{m}=b\right)$. There is some $1 \leq j \leq m-1$ such that $b_{j}=G_{0}\left(p_{\circ}\right), \underline{b}^{\prime}=\underline{b}-\left\{b_{j}\right\}$ while

$$
b_{j-1}<G_{0}\left(p_{\circ}\right)-2 / 3 \sqrt{3} \text { and } b_{j+1}>G_{0}\left(p_{\circ}\right)+2 / 3 \sqrt{3} .
$$

(3) Let $p$ and $q$ be the critical points of $G$ where $b_{j-1}<G(p)<b_{j}<G(q)<b_{j+1}$ and canceled against each other at $t=0$. The stable and unstable submanifolds $W^{s}(q)$ and $W^{u}(p)$ are transverse and intersect in a single flow line. Moreover, the neighborhood $U$ is in fact a neighborhood of

$$
\left(W^{u}(p) \cup W^{s}(q)\right) \cap G^{-1}\left[b_{j-1}, b_{j+1}\right]
$$

and $\xi^{\prime}$ coincide with $\xi$ outside $U$.

A critical point creation is the reverse of a critical point cancellation.

Suppose that the good Morse data $\mathfrak{M}^{\prime}$ is obtained from $\mathfrak{M}$ by a critical point cancellation as above. The induced parametrized Cerf decomposition $\mathfrak{C}\left(\mathfrak{M}^{\prime}\right): \mathcal{W}_{1}^{\prime} \cup \cdots \cup \mathcal{W}_{m-1}^{\prime}$ is related to $\mathfrak{C}(\mathfrak{M}): \mathcal{W}_{1} \cup \cdots \cup \mathcal{W}_{m}$ as follows. For any $i<j-1$ the parametrized elementary cobordisms $\mathcal{W}_{i}^{\prime}$ coincides with $\mathcal{W}_{i}$, while for $i \geq j$ it coincides with $\mathcal{W}_{i+1}$. Further, let $b\left(\mathbb{S}_{j}\right)$ in $\left(M_{j-1}\left(\mathbb{S}_{j}\right), T_{j-1}\right)$ denote the belt sphere of the attached handle to $\mathbb{S}_{j}$. The framed sphere $\widetilde{\mathbb{S}}_{j+1}:=d_{j}^{-1}\left(\mathbb{S}_{j+1}\right)$ intersects $b\left(\mathbb{S}_{j}\right)$ in a single point, thus there is a diffeomorphism

$$
\phi:\left(M_{j-1}, T_{j-1}\right) \rightarrow\left(M_{j-1}\left(\mathbb{S}_{j}\right)\left(\widetilde{\mathbb{S}}_{j+1}\right), T_{j-1}\right)
$$

which is unique up to isotopy and fixes $\left(M_{j-1}, T_{j-1}\right) \cap\left(M_{j-1}\left(\mathbb{S}_{j}\right)\left(\widetilde{\mathbb{S}}_{j+1}\right), T_{j-1}\right)$ (See [Cer70, Juh, Definition 2.17] and [Mil65, Theorem 5.4]). Then, $\mathcal{W}_{j}^{\prime}$ is a cobordism from $\left(M_{j-1}^{\prime}, T_{j-1}^{\prime}\right)=\left(M_{j-1}, T_{j-1}\right)$ to $\left(M_{j}^{\prime}, T_{j}^{\prime}\right)=\left(M_{j+1}, T_{j+1}\right)$ parametrized by the framed sphere $\mathbb{S}_{j}^{\prime}=\emptyset$ and the diffeomorphism $d_{j}^{\prime}$, which is isotopic to $d_{j+1} \circ \widetilde{d}_{j} \circ \phi$. Here,

$$
\widetilde{d}_{j}:\left(M_{j-1}\left(\mathbb{S}_{j}\right)\left(\widetilde{\mathbb{S}}_{j+1}\right), T_{j-1}\right) \longrightarrow\left(M_{j}\left(\mathbb{S}_{j+1}\right), T_{j}\right)
$$

is the diffeomorphism induced by $d_{j}$. For more details, see [Juh, Lemma 2.15].

Critical point switches. The Morse data $\mathfrak{M}$ and $\mathfrak{M}^{\prime}$ are related by critical point switch if $\xi=\xi^{\prime}$, $\underline{b} \backslash b_{j}=\underline{b}^{\prime} \backslash b_{j}^{\prime}$ for some $j$, and $G$ is connected to $G^{\prime}$ by a smooth family $\left\{G_{t}: W \rightarrow[a, b] \mid t \in[-1,1]\right\}$ of Morse functions which are proper for all but finitely many values of $t$ in $[-1,1]$ and $\operatorname{Crit}_{\mathcal{W}}\left(G_{t}\right)$ is independent of $t$. Furthermore, depending on the type of critical point switch, the family $\left\{G_{t}\right\}$ satisfies one of the followings:

Type I. For critical points $p, q \in \mathrm{Crit}_{W}(G)$ we have

$$
b_{j-1}<G(p)<b_{j}<G(q)<b_{j+1} \quad \text { and } \quad b_{j-1}<G^{\prime}(q)<b_{j}^{\prime}<G^{\prime}(p)<b_{j+1} .
$$

Then $G_{0}(p)=G_{0}(q)$ while $t G_{t}(p)>t G_{t}(q)$ for $t \neq 0$. Further, the family $\left\{G_{t}\right\}_{t \in[-1,1]}$ is an elementary upward or downward switch ('chemin élémentaire de croisement, ascendant or descendente' in the sense of Cerf [Cer70, Chapter II, p.40]) in a neighborhood $U$ of

$$
W_{p}^{s}(q):=W^{s}(q) \cap G^{-1}([G(p), G(q)]) \quad \text { or } \quad W_{q}^{u}(p):=W^{u}(p) \cap G^{-1}([G(p), G(q)]) .
$$

In particular, $G_{t}$ is independent of $t$ outside $U$ while $G_{t}-G$ is constant in an open neighborhood containing the critical points inside $U$. 
Type II. For critical points $p \in \operatorname{Crit}_{W}(G)$ and $q_{1}, \ldots, q_{n} \in \operatorname{Crit}(g)$ we have

$$
b_{j-1}<G(p)<b_{j}<G\left(q_{1}\right)<\cdots<G\left(q_{n}\right)<b_{j+1}
$$

while $b_{j-1}<G^{\prime}\left(q_{1}\right)<\cdots<G^{\prime}\left(q_{n}\right)<b_{j}<G^{\prime}(p)<b_{j+1}$. Then $G_{t}\left(q_{1}\right)<\cdots<G_{t}\left(q_{n}\right)<G_{t}(p)$ for some $\delta>0$ and every $1-\delta<t \leq 1$. Furthermore, the family $\left\{G_{t}\right\}_{t \in[-1,1]}$ is an elementary switch ('chemin élémentaire de croisement' in the sense of Cerf [Cer70, Chapter II, p.40]) with support in a neighborhood $U$ of

$$
W_{q}^{u}(p):=W^{u}(p) \cap G^{-1}\left(\left[G(p), G\left(q_{n}\right)\right]\right)
$$

and $\left.G_{t}\right|_{W-U}$ is independent of $t$.

Type III. There are critical points $p, q \in \operatorname{Crit}(g)$ so that $b_{j-1}<g(p)<g(q)<b_{j}, g$ has no critical value in $(g(p), g(q))$ and $b_{j-1}<g^{\prime}(q)<g^{\prime}(p)<b_{j}$. In this case $G_{0}(p)=G_{0}(q)$, while $t G_{t}(p)>t G_{t}(q)$ for $t \neq 0$. Moreover, for a neighborhood $U$ of

$$
W^{s}(q) \cap G^{-1}([G(p), G(q)]) \quad \text { or } \quad W^{u}(p) \cap G^{-1}([G(p), G(q)]),
$$

$G_{t}$ is independent of $t$ in $W-U$. Furthermore, in a neighborhood $V \subset U$ of $p$ and $q, G_{t}-G$ is constant.

If the good Morse data $\mathfrak{M}$ and $\mathfrak{M}^{\prime}$ are related by a critical point switch of type III, then it is straightforward to see that $\mathfrak{C}(\mathfrak{M})$ and $\mathfrak{C}\left(\mathfrak{M}^{\prime}\right)$ are the same. However, if they are related by a critical point switch of type I or II, then $\mathfrak{C}(\mathfrak{M})$ and $\mathfrak{C}\left(\mathfrak{M}^{\prime}\right)$ are related as in Lemma 2.16 of [Juh]. Let us recall the statement of the aforementioned Lemma (with a small modification) for a critical point switch of type II.

Lemma 4.4. (Critical point switch of type II) With the above notation fixed, if $\mathfrak{M}=(G, \underline{b}, \xi)$ and $\mathfrak{M}^{\prime}=\left(G^{\prime}, \underline{b}^{\prime}, \xi^{\prime}\right)$ are related by a critical point switch of type II, then the parametrized elementary cobordism $\mathcal{W}_{i}$ coincides with $\mathcal{W}_{i}^{\prime}$ for any $i<j-1$ and $i>j$. Moreover,

- $\mathbb{I}_{j+1} \cap d_{j}\left(b\left(\mathbb{S}_{j}\right)\right)=\emptyset$ where $b\left(\mathbb{S}_{j}\right)$ denotes the belt sphere of the attached handle to $\mathbb{S}_{j}$,

- $d_{j}\left(\mathbb{I}_{j}^{\prime}\right)=\mathbb{I}_{j+1}$ and $d_{j}^{\prime}\left(\mathbb{S}_{j}\right)=\mathbb{S}_{j+1}^{\prime}$,

- The diagram

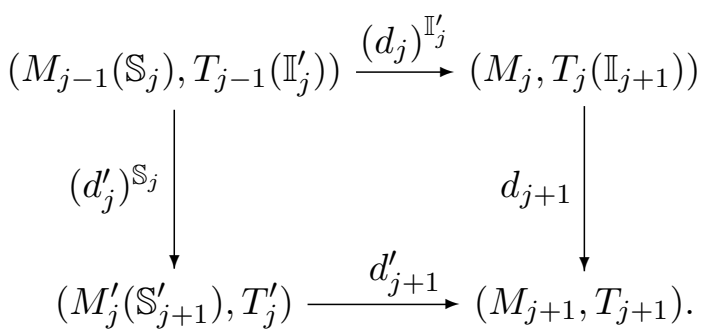

is commutative. Here, $\left(d_{j}\right)^{\mathbb{I}_{j}^{\prime}}$ and $\left(d_{j}^{\prime}\right)^{\mathbb{S}_{j}}$ are induced by $d_{j}$ and $d_{j}^{\prime}$, respectively.

Proof. See proof of Lemma 2.16 in [Juh].

Isotopy on embedded gradient-like vector field. We say that the Morse data $\mathfrak{M}=(G, \underline{b}, \xi)$ and $\mathfrak{M}^{\prime}=\left(G^{\prime}, \underline{b}^{\prime}, \xi^{\prime}\right)$ are related by doing isotopy on the embedded gradient-like vector field, if $G=G^{\prime}$ and $\underline{b}=\underline{b}^{\prime}$.

If the good Morse data $\mathfrak{M}$ and $\mathfrak{M}^{\prime}$ are related by doing isotopy on the embedded-gradient like vector fields, their corresponding parametrized Cerf decompositions, possibly after reversing some of the framed spheres, are related by ambient isotopies [Juh, Remark 2.11]. More precisely, for any $j$ there is an ambient isotopy $\left\{\phi_{t}\right\}_{t \in[0,1]}$ of $\left(M_{j-1}, T_{j-1}\right)$ with $\phi_{0}=\operatorname{Id}_{M_{j-1}}$, so that if $\mathcal{W}_{j}$ is parametrized by $\left(\mathbb{S}_{j}, d_{j}\right)$ (or $\left(\mathbb{I}_{j}, d_{j}\right)$ ) then $\phi_{1}\left(\mathbb{S}_{j}\right)=\mathbb{S}_{j}^{\prime}$ and $d_{j}^{\prime}=d_{j} \circ\left(\phi_{1}^{\prime}\right)^{-1}\left(\phi_{1}\left(\mathbb{I}_{j}\right)=\mathbb{I}_{j}^{\prime}\right.$ and $\left.d_{j}^{\prime}=d_{j} \circ\left(\phi_{1}^{\prime}\right)^{-1}\right)$. Here, $\phi_{1}^{\prime}$ is the diffeomorphism induced by $\phi_{1}$.

Adding/removing regular values. The Morse data $\mathfrak{M}=(G, \underline{b}, \xi)$ and $\mathfrak{M}^{\prime}=\left(G^{\prime}, \underline{b}^{\prime}, \xi^{\prime}\right)$ are related by adding or removing regular values, if $G=G^{\prime}$ and $\xi=\xi^{\prime}$. Thus, $\mathfrak{M}\left(\underline{b} \cup \underline{b}^{\prime}\right)=\left(G, \underline{b} \cup \underline{b}^{\prime}, \xi\right)$ is a Morse datum for $\mathcal{W}$ obtained from $\mathfrak{M}$ and $\mathfrak{M}^{\prime}$ by adding regular values. If both $\mathfrak{M}$ and $\mathfrak{M}^{\prime}$ are good, the induced parametrized Cerf decomposition $\mathfrak{C}\left(\mathfrak{M}\left(\underline{b} \cup \underline{b}^{\prime}\right)\right)$ is obtained from $\mathfrak{C}(\mathfrak{M})$ and 
$\mathfrak{C}\left(\mathfrak{M}^{\prime}\right)$ by splitting product cobordisms and cobordisms parametrized by acceptable sets of framed arcs.

Definition 4.6. We say that a parametrized Cerf decomposition $\mathfrak{C}^{\prime}$ is obtained from $\mathfrak{C}$ by a splitting, if there is some $j$ such that for any $i<j$ the parametrized elementary cobordism $\mathcal{W}_{i}^{\prime}$ coincides with $\mathcal{W}_{i}$ while for any $i>j+1$ it coincides with $\mathcal{W}_{i-1}$. Furthermore, the cobordism $\mathcal{W}_{j}$ splits as $\mathcal{W}_{j}^{\prime} \cup_{\left(M_{j}^{\prime}, T_{j}^{\prime}\right)} \mathcal{W}_{j+1}^{\prime}$ in $\mathfrak{C}^{\prime}$, such that one of $\mathcal{W}_{j}^{\prime}$ or $\mathcal{W}_{j+1}^{\prime}$, say $\mathcal{W}_{j}^{\prime}$, is either parametrized by $\left(\mathbb{S}_{j}^{\prime}=\emptyset, d_{j}^{\prime}\right)$ or $\left(\mathbb{I}_{j}^{\prime}, d_{j}^{\prime}\right)$. If $\mathcal{W}_{j}^{\prime}$ is a product then, depending on the types of $\mathcal{W}_{j+1}^{\prime}$ and $\mathcal{W}_{j}$, we have either

$$
\begin{array}{llll}
\mathbb{S}_{j}=d_{j}^{\prime-1}\left(\mathbb{S}_{j+1}^{\prime}\right) & \text { and } & d_{j}=d_{j+1}^{\prime} \circ\left(d_{j}^{\prime}\right)^{\mathbb{S}_{j}}, & \text { or } \\
\mathbb{I}_{j}=d_{j}^{\prime-1}\left(\mathbb{I}_{j+1}^{\prime}\right) & \text { and } & d_{j}=d_{j+1}^{\prime} \circ\left(d_{j}^{\prime}\right)^{\mathbb{I}_{j}} . &
\end{array}
$$

If $\mathcal{W}_{j}^{\prime}$ is parametrized by $\left(\mathbb{I}_{j}^{\prime}, d_{j}^{\prime}\right)$ then $\mathcal{W}_{j+1}^{\prime}$ is parametrized by $\left(\mathbb{I}_{j+1}^{\prime}, d_{j+1}^{\prime}\right)$. Moreover,

$$
\mathbb{I}_{j}=\mathbb{I}_{j}^{\prime} \amalg d_{j}^{\prime-1}\left(\mathbb{I}_{j+1}^{\prime}\right) \quad \text { and } \quad d_{j}=d_{j+1}^{\prime} \circ\left(d_{j}^{\prime}\right)^{\mathbb{I}_{j}-\mathbb{I}_{j}^{\prime}} .
$$

The reverse of this move, is called merging.

Therefore, for any two good Morse data $\mathfrak{M}=(G, \underline{b}, \xi)$ and $\mathfrak{M}=\left(G, \underline{b}^{\prime}, \xi\right)$ we may change $\mathfrak{C}(\mathfrak{M})$ to $\mathfrak{C}\left(\mathfrak{M}^{\prime}\right)$ by first splitting and then merging.

Left-right equivalence. Let $\mathcal{W}=(W, F)$ be a cobordism from $(M, T)$ to $\left(M^{\prime}, T^{\prime}\right)$. We say that the Morse functions $G$ and $G^{\prime}$ on $\mathcal{W}$ are related by a left-right equivalence if there are diffeomorphisms $\Phi: W \rightarrow W$ and $\phi: \mathbb{R} \rightarrow \mathbb{R}$ such that $\left.\Phi\right|_{(M, T)}=\operatorname{Id}_{(M, T)},\left.\Phi\right|_{\left(M^{\prime}, T^{\prime}\right)}=\operatorname{Id}_{\left(M^{\prime}, T^{\prime}\right)}$, and $G^{\prime}=\phi \circ G \circ \Phi^{-1}$. Moreover, we say that the good Morse data $\mathfrak{M}=(G, \underline{b}, \xi)$ and $\mathfrak{M}^{\prime}=\left(G^{\prime}, \underline{b}^{\prime}, \xi^{\prime}\right)$ are related by a left-right equivalence, if $G$ and $G^{\prime}$ are related by a left-right equivalence and under the corresponding diffeomorphisms $\underline{b}^{\prime}=\phi \circ \underline{b}$ and $\xi^{\prime}=\Phi_{\star}(\xi)$. In this case, the parametrized Cerf decomposition $\mathfrak{C}\left(\mathfrak{M}^{\prime}\right)$ is obtained from $\mathfrak{C}(\mathfrak{M})$ by a diffeomorphism equivalence. This means that $\mathcal{W}_{i}^{\prime}=\Phi\left(\mathcal{W}_{i}\right)$ as parametrized elementary cobordisms i.e. depending on the type of $\mathcal{W}_{i}, \mathbb{S}_{i}^{\prime}=\Phi\left(\mathbb{S}_{i}\right)$ or $\mathbb{I}_{i}^{\prime}=\Phi\left(\mathbb{I}_{i}\right)$ and $d_{i}^{\prime}=\Phi_{i} \circ d_{i} \circ\left(\Phi_{i-1}^{\prime}\right)^{-1}$, where $\Phi_{i}=\left.\Phi\right|_{\left(M_{i}, T_{i}\right)}$ and $\Phi_{i}^{\prime}$ is the map induced by $\Phi_{i}$ on $\left(M_{i}\left(\mathbb{S}_{i+1}\right), T_{i}\right)$ or on $\left(M_{i}, T_{i}\left(\mathbb{I}_{i+1}\right)\right)$.

4.4. Parametrized Cerf decomposition theorem. The goal of this subsection is to show that any two good Morse data associated with a stable cobordism can be related by a sequence of Cerf moves. As before, let $\mathfrak{M}=(G, \underline{b}, \xi)$ and $\mathfrak{M}^{\prime}=\left(G^{\prime}, \underline{b}^{\prime}, \xi^{\prime}\right)$ be good Morse data for $\mathcal{W}$ so that $G, G^{\prime}: W \rightarrow[a, b]$ are proper.

Lemma 4.5. If there exists a smooth family $\left\{G_{t}\right\}_{t \in[0,1]}$ of proper Morse functions on $\mathcal{W}$ with $G_{0}=G$ and $G_{1}=G^{\prime}$ then $G$ is related to $G^{\prime}$ by a left-right equivalence.

Proof. The proof is similar to the proof of Lemma 3.1 in [GWW13 with minor modifications. For every $t \in[0,1], G_{t}$ is proper. Therefore, we have a smooth family $\left\{\phi_{t}: \mathbb{R} \rightarrow \mathbb{R}\right\}_{t \in[0,1]}$ of diffeomorphisms of $\mathbb{R}$ such that $\phi_{0}=\mathrm{Id}$ and for every $t \in[0,1], \phi_{t}^{-1} \circ G_{t}$ has the same critical values as $G$. Moreover, if for $p \in \operatorname{Crit}_{\mathcal{W}}(G)$ and $p_{t} \in \operatorname{Crit}_{\mathcal{W}}\left(G_{t}\right)$ we have $\phi_{t}^{-1} G_{t}\left(p_{t}\right)=G(p)$ then either both $p$ and $p_{t}$ are critical points in $W$ or both are critical points in $F$.

Let $G_{t}^{\prime}=\phi_{t}^{-1} \circ G_{t}$. Consider a point $\left(x_{0}, t_{0}\right) \in W \times[0,1]$ such that $x_{0} \in \operatorname{Crit}_{W}\left(G_{t_{0}}^{\prime}\right)$. For sufficiently small values of $\epsilon>0$ and $\delta>0$, we can find the local coordinates $\theta_{t}: B_{\epsilon} \subset \mathbb{R}^{4} \rightarrow W$ for every $t \in\left(t_{0}-\delta, t_{0}+\delta\right)$ such that $G_{t}^{\prime} \circ \theta_{t}$ takes the normal form

$$
G_{t}^{\prime} \circ \theta_{t}\left(x_{1}, \ldots, x_{4}\right)=G_{t_{0}}^{\prime}\left(x_{0}\right)+\sum \pm x_{i}^{2} .
$$

Similarly, if $x_{0} \in \operatorname{Crit}\left(g_{t}\right)$, for sufficiently small $\epsilon, \delta>0$, we can find local coordinates $\theta_{t}: B_{\epsilon} \subset$ $\mathbb{R}^{4} \rightarrow W$ for $t \in\left(t_{0}-\delta, t_{0}+\delta\right)$, so that $F$ is given by $\left\{x_{3}=x_{4}=0\right\}$ in these coordinates and

$$
G_{t}^{\prime} \circ \theta_{t}\left(x_{1}, \ldots, x_{4}\right)=G_{t_{0}}^{\prime}\left(x_{0}\right) \pm x_{1}^{2} \pm x_{2}^{2}+x_{3} .
$$


Then, for any $(x, t)$ in a neighborhood of $\left(x_{0}, t_{0}\right)$ defined as above, let

$$
v(x, t):=\left(\left.\frac{d}{d s} \theta_{t+s}\left(\theta_{t}^{-1}(x)\right)\right|_{s=0}, 1\right) .
$$

It is straightforward to show that $\left\{G_{t}^{\prime}\right\}_{t \in[0,1]}$ is constant along the flow lines of $v(x, t)$ in this neighborhood. Consider a finite set of pairs $\left\{\left(U_{i}, v_{i}\right) \mid i=1, \ldots, n\right\}$, where each pair consists of an open neighborhoods $U_{i} \subset W \times[0,1]$ as above and the corresponding vector field $v_{i}$, such that

$$
\left\{(x, t) \in W \times[0,1] \mid x \in \operatorname{Crit}_{\mathcal{W}}\left(G_{t}^{\prime}\right)\right\} \subset \bigcup_{i=1}^{n} U_{i} .
$$

Consider an open set $U_{0} \subset W \times I$ in the complement of the critical points such that $\bigcup_{i=0}^{n} U_{i}$ covers $W \times[0,1]$. We define vector field $v_{0}$ on $U_{0}$ as

$$
v_{0}(x, t):=\left(-\left(\partial_{t} G_{t}^{\prime}\right)\left(d G_{t}^{\prime}\left(\xi_{t}\right)\right)^{-1} \xi_{t}, 1\right) .
$$

Here, $\left\{\xi_{t}\right\}_{t \in[0,1]}$ is a smooth family of vector fields on $W$ such that for every $t \in[0,1], \xi_{t}$ is an embedded gradient-like vector field for $G_{t}^{\prime}$. Note that $\left\{G_{t}^{\prime}\right\}_{t \in[0,1]}$ remain constant along the flow lines of $v_{0}$. Thus, we may patch the above local vector fields and construct a global vector field $v$ on $W \times[0,1]$ such that $\left\{G_{t}^{\prime}\right\}_{t \in[0,1]}$ remain constant along its flow lines. Hence, $G_{1}^{\prime} \circ \Phi_{1}=G$, where $\Phi_{1}$ is the time-one map of the flow of $v$. Therefore, $\phi_{1}^{-1} \circ G^{\prime} \circ \Phi_{1}=G$.

Definition 4.7. Given a stable cobordism $\mathcal{W}=(W, F)$, a proper Morse function $G$ on $\mathcal{W}$ is called almost ordered if

(1) $G$ is ordered as a Morse function on $W$, i.e. for any $p, q \in \operatorname{Crit}_{W}(G)$, $\operatorname{ind}(p)<\operatorname{ind}(q)$ implies $G(p)<G(q)$.

(2) For every $p \in \operatorname{Crit}_{W}(G)$ with ind $(p)<2, G(p)$ is smaller than the critical values of $g$, while for $p \in \operatorname{Crit}_{W}(G)$ with ind $(p)>2, G(p)$ is greater than the critical values of $g$.

The Morse function $G$ on $\mathcal{W}$ is called ordered if $G$ is almost ordered and for every $p \in \operatorname{Crit}_{W}(G)$ with $\operatorname{ind}(p)=2, G(p)$ is greater than the critical values of $g$. A good Morse datum $\mathfrak{M}=(G, \underline{b}, \xi)$ for $\mathcal{W}$ is called almost ordered if $G$ is almost ordered and it is called ordered if $G$ is ordered.

Lemma 4.6. Any good Morse datum $\mathfrak{M}=(G, \underline{b}, \xi)$ for $\mathcal{W}=(W, F)$ can be connected by a sequence of Cerf moves to an ordered good Morse datum $\mathfrak{M}^{\prime}=\left(G^{\prime}, \underline{b}^{\prime}, \xi^{\prime}\right)$ such that under these moves, the Morse function remains constant in an open neighborhood of $F$.

Proof. Consider a consecutive pair of critical points $p, q \in \operatorname{Crit}_{\mathcal{W}}(G)$ with wrong order, say $\operatorname{ind}(p)>\operatorname{ind}(q)$ while $G(p)<G(q)$. After removing the extra regular values we may assume $(G(p), G(q))$ contains exactly one $b_{j} \in \underline{b}$. Depending on the type of the critical points $p$ and $q$ one of the followings hold:

Case 1. $p, q \in \operatorname{Crit}_{W}(G)$ and $\operatorname{ind}(p)>\operatorname{ind}(q)$. By a dimension count one can show that for a generic embedded gradient-like vector field $\xi$,

$$
W^{u}(p) \cap W^{s}(q) \cap G^{-1}\left(b_{j}\right)=\emptyset .
$$

Since $\xi$ is gradient-like, for some $\delta>0$, the submanifolds $W^{u}(p)$ and $W^{s}(q)$ are disjoint from $F$ in $G^{-1}((G(p)-\delta, G(q)+\delta))$. Therefore, we have an elementary switch supported in a neighborhood of

$$
W^{u}(p) \cap G^{-1}((G(p)-\delta, G(q)+\delta)) \text { or } W^{s}(q) \cap G^{-1}((G(p)-\delta, G(q)+\delta)),
$$

and disjoint from $F$, that connects $G$ to a proper Morse function $G^{\prime}$ satisfying $G^{\prime}(q)<G^{\prime}(p)$. Furthermore, in a small neighborhood $U$ of $F$ we have $\left.G\right|_{U}=\left.G^{\prime}\right|_{U}$. Let $\xi^{\prime}=\xi$ and pick $\underline{b}^{\prime}$ such that $\underline{b}^{\prime}-b_{j}^{\prime}=\underline{b}-b_{j}$ and $G^{\prime}(q)<b_{j}^{\prime}<G^{\prime}(p)$. Then the resulted good Morse datum $\mathfrak{M}^{\prime}=\left(G^{\prime}, \underline{b}^{\prime}, \xi^{\prime}\right)$ satisfies the required conditions.

Case 2. $p \in \operatorname{Crit}(g)$ and $q \in \operatorname{Crit}_{W}(G)$, with ind $(q) \leq 1$. Let

$$
\left\{p_{1}, \ldots, p_{n_{j}}=p\right\} \subset \operatorname{Crit}(g)
$$


be the set of critical points of $g$ such that

$$
b_{j-1}<g\left(p_{1}\right)<\cdots<g\left(p_{n_{j}}\right)<b_{j} .
$$

Similar to Case 1, after changing $\xi$ to a generic embedded gradient-like vector field and by a dimension count, one can assume that

$$
\left(\bigcup_{i=1}^{n_{j}} W^{u}\left(p_{i}\right)\right) \cap W^{s}(q) \cap G^{-1}\left(b_{j}\right)=\emptyset .
$$

Moreover, for some $\delta>0, W^{s}(q)$ is disjoint from $F$ in $G^{-1}\left(\left(G\left(p_{1}\right)-\delta, G(q)\right)\right)$. Thus, as before, there is an elementary critical point switch, supported in a neighborhood of

$$
W^{s}(q) \cap G^{-1}\left(G\left(p_{1}\right)-\delta, G(q)\right),
$$

and disjoint from $F$, which changes the order of $q$ and $\left\{p_{1}, \ldots, p_{n_{j}}\right\}$. The rest of the argument is as in Case 1 with no modifications.

Case 3. $q \in \operatorname{Crit}(g)$ and $p \in \operatorname{Crit}_{W}(G)$, with ind $(p) \geq 2$. This is the same as Case 2 .

By induction on the number of pairs of critical points with wrong order, we are done.

Proposition 4.7. Let $\mathfrak{M}=(G, \underline{b}, \xi)$ and $\mathfrak{M}^{\prime}=\left(G^{\prime}, \underline{b}^{\prime}, \xi^{\prime}\right)$ be ordered, indefinite and good Morse data for a stable cobordism $\mathcal{W}=(W, F)$. Assume that on a tubular neighborhood $U$ of $F$ we have $\left.G\right|_{U}=\left.G^{\prime}\right|_{U}$ and $\left.\xi\right|_{U}=\left.\xi^{\prime}\right|_{U}$. Then $\mathfrak{M}$ can be connected to $\mathfrak{M}^{\prime}$ by a sequence of Cerf moves such that it stays indefinite and almost ordered throughout. Further, the Cerf moves can be chosen so that the Morse function and the gradient-like vector field are not changed on $U$.

Proof. Suppose $\mathcal{W}$ is a cobordism from $(M, T)$ to $\left(M^{\prime}, T^{\prime}\right)$. Let $a=\left.G\right|_{M}=\left.G^{\prime}\right|_{M}$ and $b=\left.G\right|_{M^{\prime}}=$ $\left.G^{\prime}\right|_{M^{\prime}}$. First, we show that $G$ can be connected to $G^{\prime}$ by a smooth, generic family $\left\{G_{t}\right\}_{t \in[0,1]}$, in the sense of [GK15, Definition 2.3], such that for all but finitely many values of $t$ in $[0,1], G_{t}$ is a proper, indefinite and almost ordered Morse function. Moreover, for every $t \in[0,1]$ we have $\left.G_{t}\right|_{U}=\left.G\right|_{U}=\left.G^{\prime}\right|_{U}$. The argument is similar to the proof of Theorem 4.5 in [GK15].

The Morse functions $G$ and $G^{\prime}$ coincide on $U$, so there is a generic family $\left\{G_{t}\right\}_{t \in[0,1]}$ connecting $G$ to $G^{\prime}$ such that $\left.G_{t}\right|_{U}=\left.G\right|_{U}=\left.G^{\prime}\right|_{U}$. Associated with $\left\{G_{t}\right\}$ consider a generic family of embedded gradient-like vector fields $\left\{\xi_{t}\right\}_{t \in[0,1]}$ connecting $\xi$ to $\xi^{\prime}$ such that $\left.\xi_{t}\right|_{U}$ does not depend on $t$. The family $\left\{G_{t}\right\}$ is called indefinite if for all but finitely many values of $t, G_{t}$ is an indefinite Morse function. If $\left\{G_{t}\right\}$ is not indefinite, consider $r \in[a, b]$ such that an index zero critical point is born at time $r$ in $p_{r} \in W$. Corresponding to this critical point, we have a path of critical points

$$
P=\left\{\left(t, p_{t}\right) \in[r, s] \times W \mid p_{t} \in \operatorname{Crit}_{W}\left(G_{t}\right) \text { and } \operatorname{ind}\left(p_{t}\right)=0 \text { for } t \in(r, s)\right\}
$$

in $[0,1] \times W$ such that at $p_{s}$ the index zero critical point is canceled against an index one critical point. Since $W$ is connected, for every $t \in(r, s)$ there is an index one critical point $q_{t}$ of $G_{t}$ which cancels $p_{t}$, i.e. $W^{s}\left(q_{t}\right) \cap W^{u}\left(p_{t}\right)$ is a single flow line. Therefore, for some $\delta>0$, and an ordered sequence

$$
\left(r=t_{0}<t_{1}<\cdots<t_{n}=s\right),
$$

we have paths of index one critical points

$$
Q^{i}=\left\{\left(t, q_{t}^{i}\right) \in I_{i} \times W \mid q_{t}^{i} \in \operatorname{Crit}_{W}\left(G_{t}\right)\right\}, \quad i=1, \ldots, n,
$$

such that $q_{t}^{i}$ cancels $p_{t}$ for $t \in I_{i}$, where

$$
I_{1}=\left[r, t_{1}+\delta\right), I_{2}=\left(t_{1}-\delta, t_{2}+\delta\right), \ldots, I_{n}=\left(t_{n-1}-\delta, s\right] .
$$

Moreover, $q_{r}^{1}=p_{r}$ and $q_{s}^{n}=p_{s}$. Since $\left\{\xi_{t}\right\}$ is generic, for any $1 \leq i \leq n$ and $t \in I_{i}$, the submanifold

$$
W_{P}^{s}\left(q_{t}^{i}\right)=W^{s}\left(q_{t}^{i}\right) \cap G_{t}^{-1}\left(\left[G_{t}\left(p_{t}\right), G_{t}\left(q_{t}^{i}\right)\right]\right)
$$

is disjoint from $F$. Thus we can use the Unmerge Lemma [GK15, Lemma 4.6] to cancel $P$ against $Q^{i}$ on the non-overlapping parts of the intervals $I_{i}$ for $i=2, \ldots, n-1$. Then we use either Eye 
Death Lemma [GK15, Lemma 4.7] or Swallowtail Death Lemma [GK15, Lemma 4.8] to cancel over overlaps and on the intervals $I_{1}$ and $I_{n}$.

Next, we make the family $\left\{G_{t}\right\}$ almost ordered. It is straightforward that one can use generic homotopies which pass through cusp-fold crossings to move all critical point creations before all critical point switches and all critical point cancellations after all critical point switches. See GK15, Section 2] for the exact definition of a cusp-fold crossing homotopy, see Figure 2.

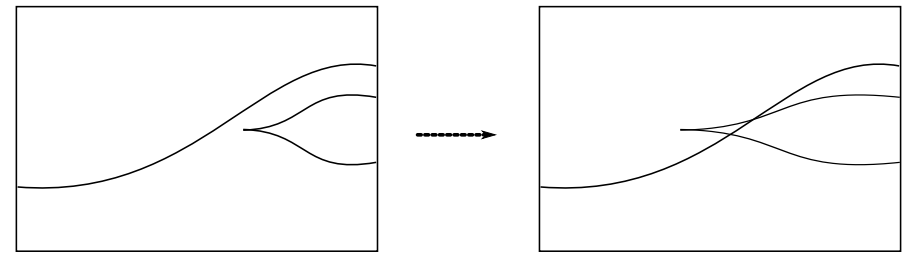

Figure 2. In a cusp-fold homotopy, the Cerf graphic, which is given by $\left\{\left(t, G_{t}(p)\right) \subset[0,1] \times[a, b] \mid p \in \operatorname{Crit}\left(G_{t}\right)\right\}$, changes as above.

Then, we modify $\left\{G_{t}\right\}$ such that if an index one/two (two/three) critical point creation/cancellation happens at a point $p_{t} \in W$ and time $t$, then $G_{t}\left(p_{t}\right)$ is larger than the values of index one (two) critical points of $G_{t}$ and smaller than the values of index two (three) critical points of $G_{t}$. Since $G$ and $G^{\prime}$ are ordered, such modifications of $\left\{G_{t}\right\}$ can be achieved through generic homotopies supported in a neighborhood of an arc disjoint from $F$.

Suppose that for paths of critical points

$$
P=\left\{\left(t, p_{t}\right) \in I_{p q} \times W \mid p_{t} \in \operatorname{Crit}_{W}\left(G_{t}\right)\right\} \quad \text { and } Q=\left\{\left(t, q_{t}\right) \in I_{p q} \times W \mid q_{t} \in \operatorname{Crit}_{W}\left(G_{t}\right)\right\}
$$

with $\operatorname{ind}(P)<\operatorname{ind}(Q)$ we have $G_{t}\left(p_{t}\right)>G_{t}\left(q_{t}\right)$ for any $t \in I_{p q}$. Further, suppose that $G_{t}$ has no critical value in $\left(G_{t}\left(p_{t}\right), G_{t}\left(q_{t}\right)\right)$. It follows from the definition of a gradient-like vector field that $W^{s}\left(p_{t}\right)$ and $W^{u}\left(q_{t}\right)$ are disjoint from $F$ in $G_{t}^{-1}\left(\left[G_{t}\left(q_{t}\right), G_{t}\left(p_{t}\right)\right]\right)$. Moreover, since $\xi_{t}$ is generic, by counting dimensions we conclude that $W^{s}\left(p_{t}\right)$ does not intersect $W^{u}\left(q_{t}\right)$. Therefore, there is a homotopy supported in a neighborhood of the 1-parameter family of descending disks $\left\{W_{Q}^{s}\left(p_{t}\right)\right\}$ or the 1-parameter family of ascending disks $\left\{W_{P}^{u}\left(q_{t}\right)\right\}$ that pulls $P$ below $Q$. Note that $W_{Q}^{s}\left(p_{t}\right)$ and $W_{P}^{u}\left(q_{t}\right)$ are defined as in Equation 4 .

The other possibility is that, for a path of index 1 critical points

$$
P=\left\{\left(t, p_{t}\right) \in I_{p} \times W \mid p_{t} \in \operatorname{Crit}_{W}(G)\right\},
$$

$g(q)<G_{t}\left(p_{t}\right)$ for some $q \in \operatorname{Crit}(g)$ and $G_{t}$ has no critical value in $\left(g(q), G_{t}\left(p_{t}\right)\right)$. As before, we can modify $\left\{G_{t}\right\}$ by a homotopy disjoint from $F$ and supported in a neighborhood of $\left\{W_{q}^{s}\left(p_{t}\right)\right\}$ to pull $P$ below $q$. Similarly, we can modify $\left\{G_{t}\right\}$ through homotopies such that for every index three critical point $p_{t}$ of $G_{t}$ (with $t \in[0,1]$ ) and every $q \in \operatorname{Crit}(g)$, we have $G_{t}\left(p_{t}\right)>g(q)$.

As a result, we obtain a generic, smooth path $\left\{G_{t}\right\}_{t \in[0,1]}$ of Morse functions which are proper, indefinite and almost ordered for all but finitely many values of $t$. Let $m$ and $m^{\prime}$ denote the smallest and largest critical values of $g$. The next step is to modify $\left\{G_{t}\right\}$ through generic homotopies such that there exists an ordered set

$$
\left(0=t_{0}<t_{1}<\cdots<t_{k}=1\right) \subset[0,1]
$$

for which $G_{t_{i}}$ is proper, indefinite and almost ordered with no critical points in $W$ above the interval $\left[m, m^{\prime}\right]$. Moreover, for any $1 \leq i \leq k$ the family $\left\{G_{t}\right\}_{t \in\left[t_{i-1}, t_{i}\right]}$ satisfies one of the followings:

(1) For every $t \in\left[t_{i-1}, t_{i}\right], G_{t}$ is a proper Morse function on $\mathcal{W}$.

(2) $\left\{G_{t}\right\}_{t \in\left[t_{i-1}, t_{i}\right]}$ corresponds to a critical point creation/cancellation connecting $G_{t_{i-1}}$ and $G_{t_{i}}$.

(3) $\left\{G_{t}\right\}_{t \in\left[t_{i-1}, t_{i}\right]}$ corresponds to switching two critical points of $G_{t_{i-1}}$ on $W$ with equal index, thus to a critical point switch of type I. 
(4) $\left\{G_{t}\right\}_{t \in\left[t_{i-1}, t_{i}\right]}$ corresponds to switching some $p \in \operatorname{Crit}_{W}\left(G_{t_{i-1}}\right)$ with all of the critical points of $g$, thus a critical point switch of type II.

In order to do so, we apply generic homotopies similar to Reidemeister II and Reidemeister III fold-crossings in [GK15, p.11, p.12] [See Figures 3 and 4]. The only difference is that we also have paths consisting of the critical points of $g$. Assume that for some $c \in[0,1]$, there are index 2 critical points $p$ and $p^{\prime}$ of $G_{c}$, such that $G_{c}(p)=G_{c}\left(p^{\prime}\right)=d$ and $d<m^{\prime}$. There is a critical point $q \in \operatorname{Crit}(g)$ so that $G_{c}$ has no critical value in the interval $(d, g(q))$. For sufficiently small $\delta>0$, consider paths of index 2 critical points

$$
\begin{aligned}
& P=\left\{\left(t, p_{t}\right) \in(c-\delta, c+\delta) \times W \mid p_{t} \in \mathrm{Crit}_{W}\left(G_{t}\right)\right\} \quad \text { and } \\
& P^{\prime}=\left\{\left(t, p_{t}^{\prime}\right) \in(c-\delta, c+\delta) \times W \mid p_{t}^{\prime} \in \operatorname{Crit}_{W}\left(G_{t}\right)\right\}
\end{aligned}
$$

so that $p=p_{c}$ and $p^{\prime}=p_{c}^{\prime}$. Since $\left\{\xi_{t}\right\}$ is generic, we may arrange for descending disks $W^{s}(p)$ and $W^{s}\left(p^{\prime}\right)$ to be disjoint from $q$. Thus, we may modify $\left\{G_{t}\right\}_{t \in(s-\delta, s+\delta)}$ through generic homotopies, disjoint from $F$, to make $G_{t}\left(p_{t}\right), G_{t}\left(p_{t}^{\prime}\right)>g(q)$ for any $t \in(c-\delta, c+\delta)$. We continue these modifications, until for any two critical point $p, p^{\prime} \in \operatorname{Crit}_{W}\left(G_{t}\right)$ with $d=G_{t}(p)=G_{t}\left(p^{\prime}\right)$ either $d<m$ or $d>m^{\prime}$.

With a similar argument, we may use generic homotopies through Reidemeister II fold-crossings, that are disjoint from $F$, and make $\left\{G_{t}\right\}_{t \in[0,1]}$ to satisfy the required conditions. Note that the family $\left\{G_{t}\right\}_{t \in[0,1]}$ remains indefinite and almost ordered under these homotopies.

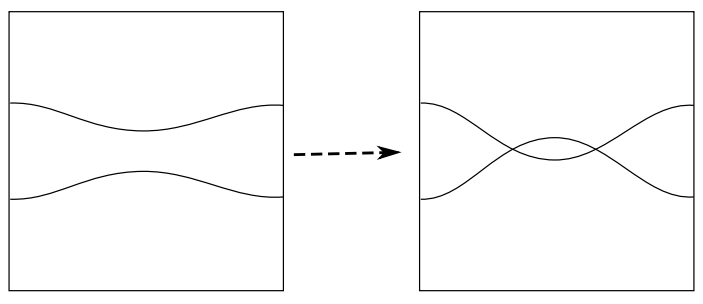

FiguRE 3. Reidemeister II fold-crossing.

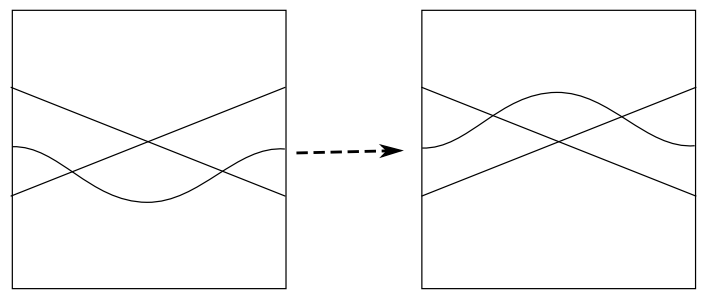

FiguRE 4. Reidemeister III fold-crossing.

For any $1 \leq i \leq k$, if $\left\{G_{t}\right\}_{t \in\left[t_{i-1}, t_{i}\right]}$ is of type (2) or (3), we may choose the interval $\left[t_{i-1}, t_{i}\right]$ sufficiently small so that we can obtain an elementary critical point creation/cancelation or critical point switch by a perturbation of $\left\{G_{t}\right\}_{t \in\left[t_{i-1}, t_{i}\right]}$.

Let us assume that an embedded gradient-like vector field $\xi_{i}$, together with an ordered set of regular values $\underline{b}_{i}$ for $G_{t_{i}}$ is given, such that $\mathfrak{M}_{i}=\left(G_{t_{i}}, \xi_{i}, \underline{b}_{i}\right)$ is a good Morse datum for $\mathcal{W}$ for some $i \in\{0,1, . ., k-1\}$. Moreover, assume that $\underline{b}_{i} \cap[m, M]=\emptyset$. Then, depending on the type of the family $\left\{G_{t}\right\}_{t \in\left[t_{i}, t_{i+1}\right]}$ we may construct a good Morse datum $\mathfrak{M}_{i+1}=\left(G_{t_{i+1}}, \xi_{i+1}, \underline{b}_{i+1}\right)$. In fact, if the family $\left\{G_{t}\right\}_{t \in\left[t_{i}, t_{i+1}\right]}$ satisfies (1), (2), (3) or (4), then $\mathfrak{M}_{i+1}$ is obtained from $\mathfrak{M}_{i}$ by a left-right equivalence, a critical point creation/cancellation, a critical point switch of type I or a critical point switch of type II, respectively.

Inductively, we get a sequence of good Morse data $\mathfrak{M}_{i}=\left(G_{i}, \xi_{i}, \underline{b}_{i}\right)$ for $i=1,2, \ldots, k$ with $G_{k}=G^{\prime}$, which is obtained by applying a sequence of Cerf moves to $\mathfrak{M}=\mathfrak{M}_{0}$. Then, $\mathfrak{M}_{k}$ is related 
to $\mathfrak{M}^{\prime}$ by isotopies of the gradient-like vector field and adding or removing regular values, and we are done.

Lemma 4.8. Let $\mathcal{W}=(W, F)$ be a stable cobordism and suppose that $g, g^{\prime}: F \rightarrow[a, b]$ are indefinite Morse functions over $F$. Then $g$ can be connected to $g^{\prime}$ by a generic family $\left\{g_{t}\right\}_{t \in[0,1]}$ of indefinite Morse functions.

Proof. This is a corollary of Theorem 4.5 in GK15.

Proposition 4.9. Let $\mathfrak{M}=(G, \underline{b}, \xi)$ be an indefinite, ordered and good Morse datum for the stable cobordism $\mathcal{W}=(W, F)$. Fix a proper, indefinite and ordered Morse function $G^{\prime \prime}: W \rightarrow\left[a^{\prime \prime}, b^{\prime \prime}\right]$ on $\mathcal{W}$. Then $\mathfrak{M}$ can be connected by a sequence of Cerf moves to a Morse datum $\mathfrak{M}^{\prime}=\left(G^{\prime}, \underline{b}^{\prime}, \xi^{\prime}\right)$, such that the Morse datum stays ordered throughout and $G^{\prime}$ coincides with $G^{\prime \prime}$ in a neighborhood of $F$.

Proof. After removing some regular values, we may assume that for some integer $n$, all critical values of $g$ lie in the interval $\left(b_{n}, b_{n+1}\right)$. Choose the regular values $m$ and $m^{\prime}$ for $G^{\prime \prime}$ such that for any $p \in \operatorname{Crit}_{W}\left(G^{\prime \prime}\right)$

$$
\left\{\begin{array}{ll}
G^{\prime \prime}(p)<m & \text { if } \operatorname{ind}(p)=1 \\
G^{\prime \prime}(p)>m^{\prime} & \text { if } \operatorname{ind}(p)=2
\end{array} .\right.
$$

Moreover, we require that every critical point $p \in F$ of $g^{\prime \prime}=\left.G^{\prime \prime}\right|_{F}$ satisfies $m<G^{\prime \prime}(p)<m^{\prime}$. Let $a=\min (\underline{b})$ and $b=\max (\underline{b})$. Consider a diffeomorphism $\phi:[a, b] \rightarrow\left[a^{\prime \prime}, b^{\prime \prime}\right]$ with

$$
\phi(a)=a^{\prime \prime}, \quad \phi(b)=b^{\prime \prime}, \quad \phi\left(b_{n}\right)=m \quad \text { and } \quad \phi\left(b_{n+1}\right)=m^{\prime} .
$$

We apply the left-right equivalence move defined by $\phi$ and $\operatorname{Id}_{W}$ on the Morse datum $(G, \underline{b}, \xi)$ to get $(\phi \circ G, \phi(\underline{b}), \xi)$. Lemma 4.8 implies that $\phi \circ g$ can be connected to $g^{\prime \prime}$ by a generic family $\left\{g_{t}\right\}_{t \in[0,1]}$ of indefinite Morse functions on $F$ which fails to be proper at the times

$$
0<c_{1}<c_{2}<\cdots<c_{l}<1 \text {. }
$$

Moreover, we may assume that for every $t \in[0,1]$ the critical values of $g_{t}$ lie in interval $\left(m, m^{\prime}\right)$. For any $1 \leq i \leq l$, consider a sufficiently small $\delta_{i}>0$ such that

$$
0<t_{1}<c_{1}<t_{2}<t_{3}<c_{2}<\cdots<t_{2 l-1}<c_{l}<t_{2 l}<1
$$

where $t_{2 i-1}=c_{i}-\delta_{i}$ and $t_{2 i}=c_{i}+\delta_{i}$. Let $p^{i}$ and $q^{i}$ denote the critical points of $g_{c_{i}}$ for which

$$
g_{c_{i}}\left(p^{i}\right)=g_{c_{i}}\left(q^{i}\right), \quad g_{2 i-1}\left(p^{i}\right)<g_{2 i-1}\left(q^{i}\right) \quad \text { and } \quad g_{2 i}\left(p^{i}\right)>g_{2 i}\left(q^{i}\right) .
$$

Here $g_{i}=g_{t_{i}}$. Since $\delta_{i}$ is sufficiently small, we may perturb $\left\{g_{t}\right\}_{t \in\left[t_{2 i-1}, t_{2 i}\right]}$ and change it to an elementary switch. In particular, there is a vector field $\widetilde{\xi}_{i}$ on $F$ which is gradient-like for any $g_{t}$ and the homotopy $\left\{g_{t}\right\}_{t \in\left[t_{2 i-1}, t_{2 i}\right]}$ is supported in a neighborhood $V_{i}$ of

$$
W^{s}\left(p^{i}\right) \cap g_{2 i-1}^{-1}\left[g_{2 i-1}\left(p^{i}\right), g_{2 i}\left(p^{i}\right)\right] \quad \text { or } \quad W^{u}\left(q^{i}\right) \cap g_{2 i-1}^{-1}\left[g_{2 i}\left(q^{i}\right), g_{2 i-1}\left(q^{i}\right)\right] \text {. }
$$

Without loss of generality, assume $V_{i}$ is a neighborhood of

$$
W^{s}\left(p^{i}\right) \cap g_{2 i-1}^{-1}\left[g_{2 i-1}\left(p^{i}\right), g_{2 i}\left(p^{i}\right)\right] \text {. }
$$

For a bump function $\omega_{i}$ supported in $V_{i}$ we have

$$
g_{t}=g_{2 i-1}+\left(t-t_{2 i-1}\right) \omega_{i} \quad \text { for } \quad t \in\left[t_{2 i-1}, t_{2 i}\right] .
$$

Furthermore, $\omega_{i}$ is constant in a neighborhood of $p^{i}$.

Let $G_{2 i-1}$ be an extension of $g_{2 i-1}$ to an ordered Morse function on $\mathcal{W}$. Consider an embedded gradient-like vector field $\xi_{i}$ for $G_{2 i-1}$ such that $\left.\xi_{i}\right|_{F}=\widetilde{\xi}_{i}$. We may extend $\omega_{i}$ to a bump function on $W$, denoted by $\Omega_{i}$ and supported in an open neighborhood $\bar{V}_{i}$ of

$$
W^{s}\left(p^{i}\right) \cap G_{2 i-1}^{-1}\left[G_{2 i-1}\left(p^{i}\right), G_{2 i}\left(p^{i}\right)\right],
$$


and is constant on a neighborhood of $p^{i}$ in $W$. Moreover, for every $t \in\left[t_{2 i-1}, t_{2 i}\right]$

$$
G_{t}=G_{2 i-1}+\left(t-t_{2 i-1}\right) \Omega_{i}
$$

is a Morse function on $\mathcal{W}$ with $\operatorname{Crit}_{\mathcal{W}}\left(G_{t}\right)=\operatorname{Crit}_{\mathcal{W}}\left(G_{2 i-1}\right)$ and corresponds to the embeddedgradient like vector field $\xi_{i}$. Thus, $\left\{G_{t}\right\}_{t \in\left[t_{2 i-1}, t_{2 i}\right]}$ is an elementary critical point switch. Further, $\mathfrak{M}_{2 i}=\left(G_{2 i}, \phi \circ \underline{b}, \xi_{i}\right)$ is an ordered, good Morse datum, obtained from $\mathfrak{M}_{2 i-1}=\left(G_{2 i-1}, \phi \circ \underline{b}, \xi_{i}\right)$ by a critical point switch of type III.

On the other hand, we may find a family

$$
\left\{\psi_{t} \mid t \in\left[t_{2 i}, t_{2 i+1}\right]\right\} \quad \text { for } i=1, \ldots, l-1,
$$

of diffeomorphisms of $\mathbb{R}$ such that $\psi_{t_{2 i}}=\mathrm{Id}$ and $\psi_{t} \circ g_{t}$ have the same critical values as $g_{2 i}$. With the same argument as in the proof of Lemma 4.5, we may define a vector field $v(x, t)$ on $F \times\left[t_{2 i}, t_{2 i+1}\right]$ such that the corresponding diffeomorphism

$$
\Psi_{t}: F \times\left\{t_{2 i}\right\} \rightarrow F \times\{t\}
$$

satisfies $\left.\psi_{t} \circ g_{t} \circ \Psi_{t}\right|_{F \times\left\{t_{2 i}\right\}}=g_{2 i}$. After choosing an arbitrary connection on a tubular neighborhood $\operatorname{nd}(F)$ of $F$ in $W$ we may extend $v$ to a vector field over $\operatorname{nd}(F) \times\left[t_{2 i}, t_{2 i+1}\right]$ and correspondingly, extend $\Psi_{t}$ from $F \times\left\{t_{2 i}\right\}$ to a diffeomorphism

$$
\Psi_{t}: \operatorname{nd}(F) \times\left\{t_{2 i}\right\} \longrightarrow \operatorname{nd}(F) \times\{t\} .
$$

Suppose that we have an extension of $g_{2 i}$ to an ordered Morse function $G_{2 i}$ on $\mathcal{W}$. Then for any $t \in\left[t_{2 i}, t_{2 i+1}\right]$ the Morse function $G_{2 i} \circ \Psi_{t}^{-1}$ is defined on the tubular neighborhood $\operatorname{nd}(F) \times\{t\} \subset W$ of $F \times\{t\}$ and has no critical points. Fix the vector field $\partial_{t}$ on the complement of $F \times\left[t_{2 i}, t_{2 i+1}\right]$. By patching the vector fields $v$ and $\partial_{t}$ using a partition of unity, we get a global vector field, still denoted by $v(x, t)$, on $W \times\left[t_{2 i}, t_{2 i+1}\right]$. The flow of $v$ defines a family of diffeomorphisms

$$
\left\{\Psi_{t}: W \times\left\{t_{2 i}\right\} \rightarrow W \times\{t\} \mid t \in\left[t_{2 i}, t_{2 i+1}\right]\right\} .
$$

Furthermore, the family

$$
\left\{G_{t}:=G_{2 i} \circ \Psi_{t}^{-1}: W \times\{t\} \rightarrow \mathbb{R} \mid t \in\left[t_{2 i}, t_{2 i+1}\right]\right\}
$$

is a family of ordered Morse function on $\mathcal{W}$ such that $\left.G_{t}\right|_{F}=\phi_{t} \circ g_{t}$. Therefore, any ordered Morse function $G_{2 i}$ on $\mathcal{W}$ with $\left.G_{2 i}\right|_{F}=g_{2 i}$ can be connected by left-right equivalence to an ordered Morse function $G_{2 i+1}$ on $\mathcal{W}$ such that $\left.G_{2 i+1}\right|_{F}=g_{2 i+1}$ and $\left.G_{2 i+1}\right|_{W-\operatorname{nd}(F)}=\left.G_{2 i}\right|_{W-\operatorname{nd}(F)}$.

We may thus connect $\mathfrak{M}=(G, \xi, \underline{b})$ to a Morse datum $\widetilde{\mathfrak{M}}=(\widetilde{G}, \widetilde{\xi}, \underline{b})$ by a sequence of Cerf moves, such that

$$
\left.\widetilde{G}\right|_{F}=\left.G^{\prime \prime}\right|_{F} \quad \text { and }\left.\quad \widetilde{G}\right|_{W-U}=\left.G\right|_{W-U}
$$

for an open neighborhood $U$ of $F$. Moreover, $\operatorname{Crit}_{W}(\widetilde{G})=\operatorname{Crit}_{W}\left(G^{\prime \prime}\right)$ is a subset of $W \backslash U$ and the Morse datum remains good, indefinite and ordered throughout.

Finally, after a small perturbation in a neighborhood of $F$, we may arrange for $t \widetilde{G}+(1-t) G^{\prime \prime}$ to be a family of Morse functions with no critical points in a neighborhood $\widetilde{U} \subset U$ of $F$. Let $v^{\prime}(x, t)$ be the corresponding vector field on $\widetilde{U} \times[0,1]$ as in the proof of Lemma 4.5. Define a global vector field $v^{\prime}$ on $W \times[0,1]$ by patching $v^{\prime}$ in $\widetilde{U}$ with the vector field $\partial_{t}$ on $(W-F) \times[0,1]$ using a partition of unity. Denote the time-one flow of $v^{\prime}$ by $\Phi_{1}$. Then, the Morse datum $\mathfrak{M}^{\prime}=\left(G^{\prime}, \underline{b}^{\prime}, \xi^{\prime}\right)$ is obtained from $\widetilde{\mathfrak{M}}$ by the left-right equivalence corresponding to $\operatorname{Id}_{\mathbb{R}}$ and $\Phi_{1}^{-1}$ (so that in particular, we have $\left.G^{\prime}=\widetilde{G} \circ \Phi_{1}^{-1}\right)$, and satisfies the required conditions.

Combining Propositions 4.7 and 4.9 , we deduce that:

Corollary 4.10. Let $\mathfrak{M}=(G, \underline{b}, \xi)$ and $\mathfrak{M}^{\prime}=\left(G^{\prime}, \underline{b}^{\prime}, \xi^{\prime}\right)$ be ordered, good and indefinite Morse data for a stable cobordism $\mathcal{W}=(W, F)$. Then, $\mathfrak{M}$ and $\mathfrak{M}^{\prime}$ can be connected by a sequence of Cerf moves. Moreover, we can keep the Morse data almost ordered, and avoid index zero and four critical points throughout the sequence. 
Lemma 4.6 and Corollary 4.10 implies the following theorem, which will be called the parametrized Cerf decomposition theorem in this paper.

Theorem 4.11. Any two good and indefinite Morse data for an stable cobordism $\mathcal{W}=(W, F)$, may be connected by a sequence of Cerf moves. Moreover, we can avoid index zero and four critical points throughout the sequence.

4.5. Ordered Morse data and parametrized decompositions. Let $\mathcal{W}=(W, F)$ be a stable cobordism from the tangle $(M, T)$ to $\left(M^{\prime}, T^{\prime}\right)$. Suppose that $G: \mathcal{W} \rightarrow[a, b]$ is an indefinite, ordered Morse function. There is an ordered set of regular values $b=\left(b_{0}=a<b_{1}<b_{2}<b_{3}=b\right)$ so that for any $p \in \operatorname{Crit}_{\mathcal{W}}(G)$, one of the following holds:

- if $p \in \operatorname{Crit}_{W}(G)$ and ind $(p)=i$ then $b_{i-1}<G(p)<b_{i}$,

- if $p \in \operatorname{Crit}(g)$ then $b_{1}<g(p)<b_{2}$.

Any such set of regular values gives a decomposition

$$
\mathcal{W}=\mathcal{W}_{1} \cup_{\left(M_{1}, T_{1}\right)} \mathcal{W}_{2} \cup_{\left(M_{2}, T_{2}\right)} \mathcal{W}_{3}
$$

where $\left(M_{i}, T_{i}\right)=\mathcal{W} \cap G^{-1}\left(b_{i}\right)$ and $\mathcal{W}_{i}=\mathcal{W} \cap G^{-1}\left(\left[b_{i-1}, b_{i}\right]\right)$. Choose a gradient-like vector field $\xi$ for $G$. It induces a parametrization on each $\mathcal{W}_{i}=\left(W_{i}, F_{i}\right)$. More precisely, for $i=1,3, \xi$ specifies a set $\mathbb{S}_{i} \subset M_{i-1} \backslash T_{i-1}$ of pairwise disjoint, framed $(i-1)$-spheres such that $W_{i}$ is diffeomorphic to $W\left(\mathbb{S}_{i}\right)$, the cobordism obtained by attaching $i$-handles to $M_{i-1}$ along $\mathbb{S}_{i}$. Moreover, it determines a diffeomorphism $d_{i}:\left(M_{i-1}\left(\mathbb{S}_{i}\right), T_{i-1}\right) \rightarrow\left(M_{i}, T_{i}\right)$. Similarly, $\xi$ determines a framed link $\mathbb{S}_{2}$ and an acceptable set of framed arcs $\mathbb{I}$ in $\left(M_{1}, T_{1}\right)$, along with a diffeomorphism $d_{2}:\left(M_{1}\left(\mathbb{S}_{2}\right), T_{1}(\mathbb{I})\right) \rightarrow\left(M_{2}, T_{2}\right)$. Any parametrized decomposition of this form is called indexed. For any indexed parametrized decomposition of $\mathcal{W}$ as above, there is an indefinite, ordered Morse function $G$, a gradient-like vector field $\xi$ and a 4 -tuple of regular values that specifies it. Any such triple $(G, \xi, b)$ is called a simplified Morse datum.

Suppose that

$$
\widetilde{\mathfrak{C}}: \mathcal{W}=\mathcal{W}_{1} \cup_{\left(M_{1}, T_{1}\right)} \mathcal{W}_{2} \cup_{\left(M_{2}, T_{2}\right)} \mathcal{W}_{3} \quad \text { and } \quad \widetilde{\mathfrak{C}}^{\prime \prime}: \mathcal{W}=\mathcal{W}_{1}^{\prime} \cup_{\left(M_{1}^{\prime}, T_{1}^{\prime}\right)} \mathcal{W}_{2}^{\prime} \cup_{\left(M_{2}^{\prime}, T_{2}^{\prime}\right)} \mathcal{W}_{3}^{\prime}
$$

are indexed parametrized decompositions for $\mathcal{W}$. For $i=1,3$, let $\left(\mathbb{S}_{i}, d_{i}\right)$ and $\left(\mathbb{S}_{i}^{\prime}, d_{i}^{\prime}\right)$ denote the parametrization of $\mathcal{W}_{i}$ and $\mathcal{W}_{i}^{\prime}$, respectively. In addition, assume $\left(\mathbb{S}_{2}, \mathbb{I}, d_{2}\right)$ and $\left(\mathbb{S}_{2}^{\prime}, \mathbb{I}^{\prime}, d_{2}^{\prime}\right)$ are the parametrization of $\mathcal{W}_{2}$ and $\mathcal{W}_{2}^{\prime}$, respectively. Then, $\widetilde{\mathfrak{C}}^{\prime}$ is obtained from $\widetilde{\mathfrak{C}}$ by creation of an index one/two critical point if:

- $\mathcal{W}_{3}$ coincides with $\mathcal{W}_{3}^{\prime}$ as parametrized cobordisms.

- $\mathbb{S}_{1}^{\prime}=\mathbb{S}_{1} \amalg s$, where $s \subset M \backslash\left(T \cup \mathbb{S}_{1}\right)$ is a framed 0-sphere,

- $\mathbb{S}_{2}^{\prime}=d_{1}^{\prime} \circ \widetilde{d}_{1}^{-1}\left(\mathbb{S}_{2}\right) \amalg k$ where $k \subset M_{1}^{\prime} \backslash T_{1}^{\prime}$ is a framed knot disjoint from $d_{1}^{\prime} \circ \widetilde{d}_{1}^{-1}\left(\mathbb{S}_{2}\right)$. Here, $\widetilde{d}_{1}: M\left(\mathbb{S}_{1}^{\prime}\right) \rightarrow M_{1}(s)$ is the diffeomorphism induced by $d_{1}$. Moreover, $\mathbb{I}^{\prime}=d_{1}^{\prime} \circ \widetilde{d}_{1}^{-1}(\mathbb{I})$

- The framed knot $\widetilde{k}=\widetilde{d}_{1} \circ\left(d_{1}^{\prime}\right)^{-1}(k)$ intersects the belt sphere of $s$ at one point. As a result, there is a corresponding diffeomorphism

$$
\phi:\left(M_{1}, T_{1}\right) \rightarrow\left(M_{1}(s)(\widetilde{k}), T_{1}\right) .
$$

Note that $\phi\left(\mathbb{S}_{2}\right)=\mathbb{S}_{2}$ and $\phi(\mathbb{I})=\mathbb{I}$.

- $d_{2}=d_{2}^{\prime} \circ D \circ \Phi$ where

$$
\begin{aligned}
& \Phi:\left(M_{1}\left(\mathbb{S}_{2}\right), T_{1}(\mathbb{I})\right) \rightarrow\left(M_{1}(s)(\widetilde{k})\left(\mathbb{S}_{2}\right), T_{1}(\mathbb{I})\right) \text { and } \\
& D:\left(M_{1}(s)(\widetilde{k})\left(\mathbb{S}_{2}\right), T_{1}(\mathbb{I})\right) \rightarrow\left(M_{1}^{\prime}\left(\mathbb{S}_{2}^{\prime}\right), T\left(\mathbb{I}^{\prime}\right)\right)
\end{aligned}
$$

are diffeomorphisms induced by $\phi$ and $d_{1}^{\prime} \circ\left(\widetilde{d}_{1}\right)^{-1}$, respectively.

The inverse of this move describes cancellation of a pair of index one/two critical points. Similarly, one may describe creation and cancellation of a pair of index two/three critical points.

Theorem 4.12. With the above notation fixed, any two indexed parametrized decompositions

$$
\widetilde{\mathfrak{C}}: \mathcal{W}=\mathcal{W}_{1} \cup_{\left(M_{1}, T_{1}\right)} \mathcal{W}_{2} \cup_{\left(M_{2}, T_{2}\right)} \mathcal{W}_{3} \quad \text { and } \quad \widetilde{\mathfrak{C}}^{\prime}: \mathcal{W}=\mathcal{W}_{1}^{\prime} \cup_{\left(M_{1}^{\prime}, T_{1}^{\prime}\right)} \mathcal{W}_{2}^{\prime} \cup_{\left(M_{2}^{\prime}, T_{2}^{\prime}\right)} \mathcal{W}_{3}^{\prime}
$$


can be connected by a sequence of the following moves:

(1) Sliding one component of $\mathbb{S}_{i}$ on another component of $\mathbb{S}_{i}$, for $i=1,2,3$,

(2) Sliding one component of $\mathbb{I}$ on another component of $\mathbb{I} \amalg \mathbb{S}_{2}$,

(3) Sliding one component of $\mathbb{S}_{2}$ on a component of $\mathbb{I}$,

(4) Creation and cancellation of index one/two or two/three critical points,

(5) Diffeomorphism equivalences.

Proof. The proof is a straightforward application of Corollary 4.10 


\section{One-handles, Three-Handles And the COBORdism MaPS}

5.1. Adding one-handles. Fix an algebra $\mathbb{A}$ over $\mathbb{F}=\mathbb{Z} / 2 \mathbb{Z}$ as before. Let $\mathcal{T}=[M, T, \mathfrak{s}, \mathfrak{u}]$ be an $\mathbb{A}$-tangle and $\mathbb{S} \subset M \backslash T$ be a framed 0-sphere. Recall that $M^{\prime}=M(\mathbb{S})$ denotes the 3-manifold obtained from $M$ after attaching a 1-handle along $\mathbb{S}$. If components of $\mathbb{S}$ lie in the same component of $M$, then $M^{\prime}$ is diffeomorphic to $M \#\left(S^{1} \times S^{2}\right)$. Otherwise, $M^{\prime}$ has one component which is the connected sum of the corresponding components of $M$. Let $T^{\prime}=T$ and $\mathfrak{u}^{\prime}=\mathfrak{u}$. It is straightforward that $\left(M^{\prime}, T^{\prime}\right)$ is a balanced tangle with $\mathbb{A}$-coloring $\mathfrak{u}^{\prime}$.

Associated with $\mathbb{S}$, we obtain a cobordim $W(\mathbb{S})$ from $M$ to $M^{\prime}$, by attaching a 1-handle along $\mathbb{S} \times\{1\}$ to $M \times[0,1]$. Let $F=T \times[0,1] \subset W(\mathbb{S})$ and regard $\mathfrak{u}$ also as a map $\mathfrak{u}_{F}: \pi_{0}(F)=\pi_{0}(T) \rightarrow \mathbb{A}$. Then, $(W(\mathbb{S}), F)$ is a stable cobordism from $(M, T)$ to $\left(M^{\prime}, T^{\prime}\right)$ and $\mathfrak{u}_{F}$ induces the $\mathbb{A}$-colorings $\mathfrak{u}$ and $\mathfrak{u}^{\prime}$ on $(M, T)$ and $\left(M^{\prime}, T^{\prime}\right)$, respectively. Given any Spin ${ }^{c}$ class $\mathfrak{t}$ on $W(\mathbb{S})$ such that $\mathfrak{s}=\left.\mathfrak{t}\right|_{M}$, we get an $\mathbb{A}$-cobordism $\mathcal{C}=\left[W(\mathbb{S}), F, \mathfrak{t}, \mathfrak{u}_{F}\right]$ from $\mathcal{T}$ to $\mathcal{T}^{\prime}=\left[M^{\prime}, T^{\prime}, \mathfrak{s}^{\prime}, \mathfrak{u}^{\prime}\right]$ where $\mathfrak{s}^{\prime}=\left.\mathfrak{t}\right|_{M^{\prime}}$. In fact, the $\operatorname{Spin}^{c}$ structure $\mathfrak{s}$ always determines $\mathfrak{t}$ and thus $\mathfrak{s}^{\prime}$. Let $\mathcal{C}(\mathbb{S})=\mathcal{C}$ and $\mathcal{T}(\mathbb{S})=\mathcal{T}^{\prime}$.

We may choose a Heegaard surface $\Sigma$ for $(M, T)$ so that it cuts each one of the two disjoint balls in a disk. Denote the boundary curves of these two disks by $C_{1}$ and $C_{2}$, and their centers by $w_{1}$ and $w_{2}$, respectively. Further, each connected component $T_{i}$ of $T$ cuts $\Sigma$ in a single transverse point $z_{i}$, let $\mathbf{z}=\left\{z_{1}, \ldots, z_{\ell}\right\}$. For appropriate collections $\boldsymbol{\alpha}$ and $\boldsymbol{\beta}$ of pairwise disjoint circles on $\Sigma \backslash \mathbf{z}$ that bound disks on the two sides of $\Sigma$ in $M \backslash T$, we get a Heegaard diagram

$$
\mathcal{H}=\left(\Sigma, \boldsymbol{\alpha}, \boldsymbol{\beta}, \mathfrak{u}: \mathbf{z}=\left\{z_{1}, \ldots, z_{\ell}\right\} \rightarrow \mathbb{A}, \mathfrak{s}\right)
$$

for $\mathcal{T}$. In this situation, a Heegaard diagram for $\mathcal{T}^{\prime}$ may be constructed as follows. There is a properly embedded cylinder $S$ on the one-handle attached to $M$ with boundary circles $C_{1}$ and $C_{2}$. If we remove the two disks with centers $w_{1}$ and $w_{2}$ from $\Sigma$ and glue $S$ to the resulting surface (which has $C_{1}$ and $C_{2}$ as its two boundary components) we obtain a Heegaard surface $\Sigma^{\prime}$ for $M^{\prime}$. Let $\alpha$ and $\beta$ be circles on $S$ which bound disks on the two sides of $\Sigma^{\prime}$, and cut each other in a pair of canceling intersection points, i.e. are Hamiltonian isotopes of each other. Then,

$$
\left(\Sigma^{\prime}, \boldsymbol{\alpha}^{\prime}=\boldsymbol{\alpha} \cup\{\alpha\}, \boldsymbol{\beta}^{\prime}=\boldsymbol{\beta} \cup\{\beta\}, \mathbf{z}\right)
$$

is a Heegaard diagram for $\left(M^{\prime}, T^{\prime}\right)$. Moreover, if $\mathcal{H}$ is $\mathfrak{s}$-admissible in the strong sense of [AE15, Remark 4.6], the diagram

$$
\mathcal{H}^{\prime}=\left(\Sigma^{\prime}, \boldsymbol{\alpha}^{\prime}, \boldsymbol{\beta}^{\prime}, \mathfrak{u}: \mathbf{z} \rightarrow \mathbb{A}, \mathfrak{s}^{\prime}\right)
$$

is a Heegaard diagram for $\mathcal{T}^{\prime}$. We will thus assume the above stronger form of admissibility for the Heegaard diagram $\mathcal{H}$.

The union of the cylinder $S$ and the aforementioned disks with centers $w_{1}$ and $w_{2}$ is a sphere, which will be denoted by $\bar{S}$. We may label the two intersection points between $\alpha$ and $\beta$ by $\theta_{\alpha \beta}$ and $\theta_{\beta \alpha}$, so that the bigons on the cylinder $S$ connect $\theta_{\alpha \beta}$ to $\theta_{\beta \alpha}$ as the domains of the Whitney disks for the Heegaard diagram $H_{S}=\left(\bar{S}, \alpha, \beta, w_{1}, w_{2}\right)$.

With the above two Heegaard diagrams fixed, and given an $\mathbb{A}$-module $\mathbb{M}$, we construct a cobordism map from $\operatorname{HF}^{\mathbb{M}}(\mathcal{T})$ to $\operatorname{HF}^{\mathbb{M}}\left(\mathcal{T}^{\prime}\right)$ associated with adding the 1-handle along the framed 0-sphere $\mathbb{S}$. A completely similar construction would give a cobordism map associated with attaching a 3handle along a framed 2-sphere.

5.2. Stretching the necks in Heegaard diagrams. The construction of the cobordism map for 1-handles rests on a slight generalization of [OS08, Theorem 5.1], which will be discussed in the present subsection.

Proposition 5.1. Fix the Heegaard diagrams

$$
\mathcal{H}^{i}=\left(\Sigma^{i}, \boldsymbol{\alpha}^{i}, \boldsymbol{\beta}^{i}, \mathbf{z}^{i}\right), \quad i=1,2,
$$

with extra marked points $\mathbf{w}^{i}=\left\{w_{1}^{i}, \ldots, w_{l}^{i}\right\}$ on $\Sigma^{i} \backslash\left(\boldsymbol{\alpha}^{i} \cup \boldsymbol{\beta}^{i} \cup \mathbf{z}^{i}\right)$ for $i=1,2$. Let $\Sigma$ denote the surface obtained from $\Sigma^{1}$ and $\Sigma^{2}$ by attaching $l$ one-handles (necks) which connect $w_{j}^{1}$ to $w_{j}^{2}$, for $j=1, \ldots, l$. Denote the number of curves in $\boldsymbol{\alpha}^{i}$ and $\boldsymbol{\beta}^{i}$ by $d^{i}$ and the genus of $\Sigma^{i}$ by $g^{i}$. For 
$i=1,2$, choose a Whitney disk $\phi^{i}$ for $\mathcal{H}^{i}$, with $n_{w_{j}^{1}}\left(\phi^{1}\right)=n_{w_{j}^{2}}\left(\phi^{2}\right)=k_{j}$ and let $\phi=\phi^{1} \star \phi^{2}$ be the homotopy class of the Whitney disk obtained by joining $\phi^{1}$ and $\phi^{2}$ along the necks.

(1) $\mu(\phi)=\mu\left(\phi^{1}\right)+\mu\left(\phi^{2}\right)-2\left(k_{1}+\cdots+k_{l}\right)$.

(2) Let $J_{t}$ be a path of almost-complex structures on $\Sigma$, which are stretched along the necks, so that all necks have length $t$, and as $t \rightarrow \infty$ converges to a degenerate path of almost complex structures on

$$
\Sigma^{1} \vee \Sigma^{2}=\frac{\Sigma_{1} \amalg \Sigma_{2}}{\left\{w_{i}^{1} \sim w_{i}^{2} \mid i=1, \ldots, l\right\}}
$$

giving the path $J^{i}$ of almost complex structures on $\Sigma^{i}$. If for any $R \in \mathbb{R}$, there is some $t>R$ so that the moduli space $\mathcal{M}(\phi)$ is non-empty for $J_{t}$, then the moduli spaces of broken pseudo-holomorphic flowlines representing $\phi^{1}$ and $\phi^{2}$ are non-empty.

(3) Suppose $\mathcal{H}^{i}$ and $\phi^{i}$ satisfy the followings:

(a) $\mu\left(\phi^{1}\right)=1, \mu\left(\phi^{2}\right)=2\left(k_{1}+\cdots+k_{l}\right)$ and $\mathfrak{u}\left(\phi^{1}\right) \neq 0$,

(b) All components of $\Sigma^{2} \backslash \boldsymbol{\alpha}^{2}$ and $\Sigma^{2} \backslash \boldsymbol{\beta}^{2}$ are punctured spheres and $d^{2}>g^{2}$.

If $J^{1}$ and $J^{2}$ are generic, then for sufficiently large $t$, the moduli space $\mathcal{M}(\phi)$ may be identified with the fiber product

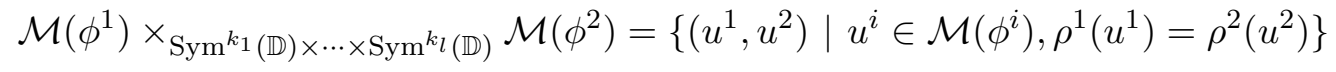

where $\rho^{i}=\rho_{1}^{i} \times \cdots \times \rho_{l}^{i}$ denotes the product of evaluation maps

$$
\rho_{j}^{i}=\rho_{w_{j}^{i}}: \mathcal{M}\left(\phi^{i}\right) \longrightarrow \operatorname{Sym}^{k_{j}}(\mathbb{D}), \quad \rho_{j}^{i}(u)=u^{-1}\left(\left\{w_{j}^{i}\right\} \times \operatorname{Sym}^{d_{j}-1}\right) .
$$

Proof. The proof of Proposition 5.1 is basically the same as the proof of [OS08, Theorem 5.1], and uses the cylindrical reformulation of Heegaard Floer homology by Lipshitz [Lip06]. We only need to make a small modification to the last part of the argument of Ozsváth and Szabó. In fact, the proof of the first two claims remains unchanged. We will thus focus on the proof of the last claim.

Consider a sequence $\left\{t_{j}\right\}$ of real numbers which converges to $\infty$ such that $\mathcal{M}(\phi)$ is nonempty for each $J_{t_{j}}$. In Lipshitz reformulation, as we stretch the necks (i.e. as $j \rightarrow \infty$ ), every sequence $\left\{v_{j}\right\}_{j \in \mathbb{Z}^{+}}$of curves with $v_{j} \in \mathcal{M}_{J_{t_{j}}}(\phi)$ has a subsequence which converges to a pseudo holomorphic curve in the symplectic manifold

$$
\begin{aligned}
W(\infty) & =\left(\left(\Sigma^{1}-\mathbf{w}^{1}\right) \times[0,1] \times \mathbb{R}\right) \coprod\left(\left(\Sigma^{2}-\mathbf{w}^{2}\right) \times[0,1] \times \mathbb{R}\right) \\
& =\left(W^{1}-\mathbf{w}^{1} \times[0,1] \times \mathbb{R}\right) \coprod\left(W^{2}-\mathbf{w}^{2} \times[0,1] \times \mathbb{R}\right) .
\end{aligned}
$$

which may be completed to a $J^{1}$-holomorphic curve in the symplectic manifold $W^{1}=\Sigma^{1} \times[0,1] \times \mathbb{R}$ and a $J^{2}$-holomorphic curve in $W^{2}=\Sigma^{2} \times[0,1] \times \mathbb{R}$. The components of this limit consist of preglued flowlines, boundary degenerations and nodal curves supported entirely inside the fibers of the projection map to $\mathbb{D} \simeq[0,1] \times \mathbb{R}$, which are identified with $\Sigma^{1} \vee \Sigma^{2}$. By ignoring the matching conditions for the pre-glued flowlines we obtain the representative in $\mathcal{M}\left(\phi^{1}\right)$ and $\mathcal{M}\left(\phi^{2}\right)$ (in fact, this proves the second claim).

Let us now assume that $\mu\left(\phi^{1}\right)=1$. Since $\mathfrak{u}\left(\phi^{1}\right) \neq 0$, in the above Gromov limit we obtain a $J^{1}$-holomorphic representative $u^{1}$ of $\phi^{1}$ and possibly a broken flow-line representative of $\phi^{2}$. If this latter representative has no closed components, a component $u^{2}$ of it forms a pre-glued flowline together with $u^{1}$. Since other possible components of $\phi^{2}$ (including the boundary degenerations) each correspond to a positive share of the Maslov index, it follows that $\mu\left(u^{2}\right) \leq 2\left(k_{1}+\cdots+k_{l}\right)$, with equality happening only if $u^{2}$ represents $\phi^{2}$ and there are no other components. For a generic point

$$
\Delta=\rho^{1}\left(u^{1}\right) \in \operatorname{Sym}^{k_{1}}(\mathbb{D}) \times \cdots \times \operatorname{Sym}^{k_{l}}(\mathbb{D})
$$

the moduli space $\left(\rho^{2}\right)^{-1}(\Delta)$, which contains $u^{2}$, is of expected dimension $\mu\left(u^{2}\right)-2\left(k_{1}+\cdots+k_{l}\right)$. Thus, $u^{2}$ is forced to represent $\phi^{2}$ if $J^{1}$ (and thus $\Delta$ ) is generic. 
The difference with the argument of Ozsváth and Szabó appears when we consider the possibility of having closed components in the broken flowline representing $\phi^{2}$. Let us assume that these closed components represent $m\left[\Sigma^{2}\right]$. After deleting these components we obtain a new class $\psi^{2}$, represented by $u^{2}$, with

Moreover, each component $\Delta_{j}^{\prime}$ of

$$
\mu\left(\psi^{2}\right)=\mu\left(\phi^{2}\right)-2 m\left(d^{2}-g^{2}+1\right) .
$$

$$
\rho^{2}\left(u^{2}\right)=\Delta^{\prime}=\left(\Delta_{1}^{\prime}, \ldots, \Delta_{l}^{\prime}\right) \in \operatorname{Sym}^{k_{1}}(\mathbb{D}) \times \cdots \times \operatorname{Sym}^{k_{l}}(\mathbb{D})
$$

is obtained from the component $\Delta_{j}$ of $\rho^{1}\left(u^{1}\right)=\Delta$ by deleting $m$ points $\left\{p_{1}, \ldots, p_{m}\right\}$ from it. Note that these $m$ points are determined by the projection of the closed components in $W^{2}$ over $\mathbb{D}$, and are thus the same for $\Delta_{1}, \ldots, \Delta_{l}$. This means that the components of $\Delta$ have the points $p_{1}, \ldots, p_{m}$ in common. The subset of $\mathcal{M}\left(\phi^{1}\right)$ consisting of $J^{1}$-holomorphic curves satisfying this condition is of expected dimension

$$
\mu\left(\phi^{1}\right)-2 m(l-1)=1-2 m(l-1) .
$$

If $m>0, l>1$, and $J^{1}$ is generic, this subset of $\mathcal{M}\left(\phi^{1}\right)$ is empty. The only remaining case is thus the case where $l=1$ and $m>0$.

In this latter case, the argument of Ozsváth and Szabó may be used. The moduli space of all $u^{2} \in \mathcal{M}\left(\phi^{2}\right)$ with $\rho^{2}\left(u^{2}\right)=\Delta^{\prime}$ is of expected dimension

$$
\mu\left(\phi_{2}\right)-2\left(k_{1}-m\right)=2 k_{1}-2 m\left(d_{2}+1-g_{2}\right)-2\left(k_{1}-m\right)=-2 m\left(d_{2}-g_{2}\right)<0
$$

and hence is empty for a generic choice of $J^{1}$ and $J^{2}$.

It follows that every weak limit of the curves in $\mathcal{M}(\phi)$, as we stretch the necks, is in correspondence with a pre-glued curve in

$$
\left\{\left(u^{1}, u^{2}\right) \mid u^{i} \in \mathcal{M}\left(\phi^{i}\right), \rho^{1}\left(u^{1}\right)=\rho^{2}\left(u^{2}\right)\right\}=\mathcal{M}\left(\phi^{1}\right) \times_{\operatorname{Sym}^{k_{1}}(\mathbb{D}) \times \cdots \times \operatorname{Sym}^{k_{l}(\mathbb{D})}} \mathcal{M}\left(\phi^{2}\right) .
$$

Moreover, from a pre-glued curve we may obtain an actual $J_{t_{j}}$-holomorphic curve if $j$ is sufficiently large. This completes the proof.

5.3. Construction of the cobordism map. Choose a generic path $J$ of almost complex structures on the Heegaard surface $\Sigma$. We identify $\left(\bar{S}, w_{1}, w_{2}\right)$ with $\left(\mathbb{P}^{1}, 0, \infty\right)$ and denote the induced complex structure on $\bar{S}$ by $J_{S}$. Let $J^{\prime}$ be a generic path of almost-complex structures on $\Sigma^{\prime}$ which is sufficiently close to the join of $J$ and $J_{S}$.

Let $f_{\mathbb{S}}: \mathrm{CF}_{J}(\mathcal{H}) \rightarrow \mathrm{CF}_{J^{\prime}}\left(\mathcal{H}^{\prime}\right)$ be the homomorphism defined as

$$
f_{\mathbb{S}}(\mathbf{x}):=\mathbf{x} \times\left\{\theta_{\alpha \beta}\right\} \in \mathrm{CF}_{J^{\prime}}\left(\mathcal{H}^{\prime}\right)
$$

for any generator $\mathbf{x} \in \mathbb{T}_{\alpha} \cap \mathbb{T}_{\beta}$ representing the $\operatorname{Spin}^{c}$ class $\mathfrak{s}$.

Proposition 5.2. The homomorphism $f_{\mathbb{S}}$ is a chain map.

Proof. Let $\mathbf{x} \in \mathbb{T}_{\alpha} \cap \mathbb{T}_{\beta}$ be an intersection point corresponding to the $\operatorname{Spin}^{c}$ class $\mathfrak{s} \in \operatorname{Spin}^{c}(M)$. Suppose

$$
\mathbf{y} \times \theta \in \mathbb{T}_{\alpha^{\prime}} \cap \mathbb{T}_{\beta^{\prime}}=\left(\mathbb{T}_{\alpha} \cap \mathbb{T}_{\beta}\right) \times(\alpha \cap \beta)
$$

is a generator contributing to $d f_{\mathbb{S}}(\mathbf{x})$, via $u^{\prime} \in \mathcal{M}\left(\phi^{\prime}\right)$, where $\phi^{\prime} \in \pi_{2}\left(\mathbf{x} \times \theta_{\alpha \beta}, \mathbf{y} \times \theta\right)$. Thus, $\mu\left(\phi^{\prime}\right)=1$ and $\mathfrak{u}^{\prime}\left(\phi^{\prime}\right) \neq 0$. The class $\phi^{\prime}$ is the join of classes $\phi \in \pi_{2}(\mathbf{x}, \mathbf{y})$ (corresponding to the Heegaard diagram $\mathcal{H}$ ) and $\phi_{S} \in \pi_{2}\left(\theta_{\alpha \beta}, \theta\right)$ (corresponding to the Heegaard diagram $\mathcal{H}_{S}$ ), along a pair of necks corresponding to $w_{1}$ and $w_{2}$ which connect the two Heegaard diagrams. Further, $\mathfrak{u}^{\prime}\left(\phi^{\prime}\right) \neq 0$ implies that $\mathfrak{u}(\phi) \neq 0$.

Let $|\theta|$ denote the number of intersection points in $\theta$ which are different from $\theta_{\alpha \beta}$. Thus, $|\theta| \in$ $\{0,1\}$. For a class $\phi^{\prime}$ as above with $\mu\left(\phi^{\prime}\right)=1$, we have

$$
1=\mu\left(\phi^{\prime}\right)=\mu(\phi)+\mu\left(\phi_{S}\right)-2 n_{w_{1}}\left(\phi_{S}\right)-2 n_{w_{2}}\left(\phi_{S}\right)=\mu(\phi)+|\theta| .
$$


Since the path $J^{\prime}$ of almost complex structures is chosen close to the join of $J$ and $J_{S}$, and $\mathfrak{u}(\phi) \neq 0$, Proposition 5.1 implies that every pseudo-holomorphic representative $u^{\prime}$ of $\phi^{\prime}$ is in correspondence with a $J$-holomorphic curve $u \in \mathcal{M}(\phi)$ and a $J_{S^{-}}$-holomorphic curve $u_{S} \in \mathcal{M}\left(\phi_{S}\right)$, which are pre-glued.

If $\theta \neq \theta_{\alpha \beta}$, then $\mu(\phi)=0$ and $u$ is the constant map. It follows that $n_{w_{i}}\left(\phi_{S}\right)=n_{w_{i}}(\phi)=0$ and $\phi_{S}$ corresponds to one of the two bigon connecting $\theta_{\alpha \beta}$ to $\theta_{\beta \alpha}$. The total contribution of such $u^{\prime}$ to $d f_{\mathbb{S}}(\mathbf{x})$ is thus zero.

The remaining contributions to $d f_{\mathbb{S}}(\mathbf{x})$ come from the classes $\phi^{\prime}$ such that $\mu(\phi)=1$, while $\theta=\theta_{\alpha \beta}$ and $n_{w_{i}}\left(\phi_{S}\right)=n_{w_{i}}(\phi)=k_{i}$ for $i=1,2$. We would like to show that the total contribution to $d f_{\mathbb{S}}(\mathbf{x})$ from such $u^{\prime}$ is equal to the corresponding contribution from $u \in \mathcal{M}(\phi)$ to $d(\mathbf{x})$. It follows from Proposition 5.1, that if the necks connecting $S$ to $\Sigma$ are sufficiently stretched, then for $\phi^{\prime}=\phi \star \phi_{S}$ the moduli space $\mathcal{M}\left(\phi^{\prime}\right)$ maybe identified with the fiber product $\mathcal{M}(\phi) \times_{\mathrm{Sym}^{k_{1}}(\mathbb{D}) \times \operatorname{Sym}^{k_{2}(\mathbb{D})}} \mathcal{M}\left(\phi_{S}\right)$. In particular, any $u^{\prime} \in \mathcal{M}\left(\phi^{\prime}\right)$ corresponds to the degeneration $u \star u_{S}$ with $u \in \mathcal{M}(\phi)$ and $u_{S} \in \mathcal{M}\left(\phi_{S}\right)$ such that

where

$$
\rho_{\Sigma}(u)=\rho_{S}\left(u_{S}\right) \in \operatorname{Sym}^{k_{1}}(\mathbb{D}) \times \operatorname{Sym}^{k_{2}}(\mathbb{D}),
$$

$$
\begin{aligned}
& \rho_{\Sigma}=\rho_{w_{1}} \times \rho_{w_{2}}: \mathcal{M}(\phi) \rightarrow \operatorname{Sym}^{k_{1}}(\mathbb{D}) \times \operatorname{Sym}^{k_{2}}(\mathbb{D}) \text { and } \\
& \rho_{S}=\rho_{w_{1}} \times \rho_{w_{2}}: \mathcal{M}\left(\phi_{S}\right) \rightarrow \operatorname{Sym}^{k_{1}}(\mathbb{D}) \times \operatorname{Sym}^{k_{2}}(\mathbb{D})
\end{aligned}
$$

are the evaluation maps.

This reduces the proof to the first part of the following Lemma, which will be proved in Subsection 5.4. With this lemma in place, the proof of the proposition is complete.

Lemma 5.3. Let $\alpha, \beta, \gamma$ denote three curves on $\mathbb{P}^{1}$ which are small Hamiltonian isotopes of one another and denote the corresponding top intersection points by $\theta_{a} \in \beta \cap \gamma, \theta_{b} \in \alpha \cap \gamma$ and $\theta_{c} \in \alpha \cap \beta$. Let $w_{1}$ and $w_{2}$ denote markings on the two domains in the complement of the isotopy regions. Let $\phi_{k_{1}, k_{2}}$ denote the set of Whitney disks $\phi \in \pi_{2}\left(\theta_{c}, \theta_{c}\right)$ with $n_{w_{i}}(\phi)=k_{i}, i=1,2$ and $\Delta_{k_{1}, k_{2}}$ denote the union of the triangle classes $\Delta \in \pi_{2}\left(\theta_{c}, \theta_{a}, \theta_{b}\right)$ with $n_{w_{i}}(\Delta)=k_{i}, i=1,2$. Then for generic

$$
\left(p_{1}, p_{2}\right) \in \operatorname{Sym}^{k_{1}}(\mathbb{D}) \times \operatorname{Sym}^{k_{2}}(\mathbb{D})
$$

and a generic path of almost complex structures on $\mathbb{P}^{1}$ we have

(i) $n_{p_{1}, p_{2}}\left(\phi_{k_{1}, k_{2}}\right):=\#\left\{u \in \mathcal{M}\left(\phi_{k_{1}, k_{2}}\right) \mid \rho_{w_{i}}(u)=p_{i}, i=1,2\right\}=1$ and

(ii) $n_{p_{1}, p_{2}}\left(\Delta_{k_{1}, k_{2}}\right):=\#\left\{u \in \mathcal{M}\left(\Delta_{k_{1}, k_{2}}\right) \mid \rho_{w_{i}}(u)=p_{i}, i=1,2\right\}=1$.

5.4. Proof of Lemma 5.3. The proof is closely related to the proof of [OS08, Lemma 6.4]. We will prove the second claim. The first claim is in fact easier, and its proof is completely similar. The first step is to show that the number $n_{p_{1}, p_{2}}\left(\Delta_{k_{1}, k_{2}}\right)$ does not depend on the generic choice of $\left(p_{1}, p_{2}\right)$. Given a generic path $\left\{\left(p_{1}^{t}, p_{2}^{t}\right)\right\}_{t \in[0,1]}$ in $\operatorname{Sym}^{k_{1}}(\mathbb{D}) \times \operatorname{Sym}^{k_{2}}(\mathbb{D})$ connecting the generic points $\left(p_{1}^{0}, p_{2}^{0}\right)$ and $\left(p_{1}^{1}, p_{2}^{1}\right)$, consider the moduli space

$$
\left\{(u, t) \mid t \in[0,1], u \in \mathcal{M}\left(\Delta_{k_{1}, k_{2}}\right), \rho_{w_{i}}(u)=p_{i}^{t}, i=1,2\right\},
$$

which is a smooth 1-dimensional moduli space with ends determined by the Gromov limits of its points. Picture $\mathbb{D}$ as a $Y$ shape domain, and assume that the complex structure is translation invariant as we move towards infinity in any of three directions corresponding to $v_{a}, v_{b}$ and $v_{c}$. Three types of the boundary points correspond to a degenerations of the domain into a disk $\phi$ and a triangle class $\Delta_{\phi}$. Since the path $\left\{\left(p_{1}^{t}, p_{2}^{t}\right)\right\}_{t}$ remains in a compact subset of the domain, the disk $\phi$ can not contain any of the pre-images of $w_{1}$ or $w_{2}$, i.e. $n_{w_{1}}(\phi)=n_{w_{2}}(\phi)=0$. There are no holomorphic disks to $\theta_{b}$ with coefficient 0 at $w_{1}$ and $w_{2}$. Thus, $\phi$ corresponds to the disks contributing to $\widehat{\partial}\left(\theta_{a}\right)$ or $\widehat{\partial}\left(\theta_{c}\right)$ which are all zero, i.e. the total number of such ends is always zero. The remaining ends correspond to

$$
-\left\{u \in \mathcal{M}\left(\Delta_{k_{1}, k_{2}}\right) \mid \rho_{w_{i}}(u)=p_{i}^{0}, i=1,2\right\} \coprod\left\{u \in \mathcal{M}\left(\Delta_{k_{1}, k_{2}}\right) \mid \rho_{w_{i}}(u)=p_{i}^{1}, i=1,2\right\},
$$


implying the independence of $n_{p_{1}, p_{2}}\left(\Delta_{k_{1}, k_{2}}\right)$ from the generic choice of $\left(p_{1}, p_{2}\right)$ in the product $\operatorname{Sym}^{k_{1}}(\mathbb{D}) \times \operatorname{Sym}^{k_{2}}(\mathbb{D})$.

Consider one of the branches of the $Y$-shape domain $\mathbb{D}$, for instance the one corresponding to $v_{a}$, and identify the branch with $[0,1] \times(0, \infty)$. Denote the projection over the second factor by $\pi_{\mathbb{R}}$. Choose the generic path $\left(p_{1}^{T}, p_{2}^{T}\right)$ of points in $\operatorname{Sym}^{k_{1}}(\mathbb{D}) \times \operatorname{Sym}^{k_{2}}(\mathbb{D})$ so that $p_{1}^{T}$ is a union of $k_{1}$ points $p_{1,1}^{T}, \ldots, p_{1, k_{1}}^{T}$ in the above branch so that

$$
\pi_{\mathbb{R}}\left(p_{1,1}^{T}\right)>T \text { and } \pi_{\mathbb{R}}\left(p_{1, i+1}^{T}\right)-\pi_{\mathbb{R}}\left(p_{1, i}^{T}\right)>T .
$$

Similarly, assume that $p_{2}^{T}$ is a union of $k_{2}$ points $p_{2,1}^{T}, \ldots, p_{2, k_{2}}^{T}$ in the above branch so that

$$
\pi_{\mathbb{R}}\left(p_{2,1}^{T}\right)-\pi_{\mathbb{R}}\left(p_{1, k_{1}}^{T}\right)>T \text { and } \pi_{\mathbb{R}}\left(p_{2, i+1}^{T}\right)-\pi_{\mathbb{R}}\left(p_{2, i}^{T}\right)>T
$$

One may then consider the ends of the smooth 1-dimensional moduli space

$$
\mathcal{N}=\coprod_{T \in[1, \infty)}\left\{u \in \mathcal{M}\left(\Delta_{k_{1}, k_{2}}\right) \mid \rho_{w_{i}}(u)=p_{i}^{T}, i=1,2\right\} .
$$

Two types of boundary ends correspond to degenerations of $u$ to a triangle and a disk $\phi$ which goes to $\theta_{b}$ or starts from $\theta_{c}$. Since the path of points remains in a compact subset of the branch corresponding to vertices $v_{b}$ and $v_{c}$, we find $n_{w_{1}}(\phi)=n_{w_{2}}(\phi)=0$. There are no holomorphic disks to $\theta_{b}$ with coefficients 0 at $w_{1}$ and $w_{2}$, and the disks $\phi$ which contribute to $\widehat{\partial}\left(\theta_{c}\right)$ come in canceling pairs. Such pairs correspond to pairs of points in the boundary of $\mathcal{N}$ with canceling contributions. The remaining boundary components of $\mathcal{N}$ are in correspondence with the points in union of

$$
-\left\{u \in \mathcal{M}\left(\Delta_{k_{1}, k_{2}}\right) \mid \rho_{w_{i}}(u)=p_{i}^{1}, i=1,2\right\}
$$

and the product

$$
\mathcal{M}\left(\Delta_{0,0}\right) \times\left(\prod_{i=1}^{k_{1}}\left\{u \in \mathcal{M}(\phi) \mid \rho_{w_{1}}(u)=t_{1, i}\right\}\right) \times\left(\prod_{i=1}^{k_{2}}\left\{u \in \mathcal{M}(\psi) \mid \rho_{w_{1}}(u)=t_{2, i}\right\}\right) .
$$

Here $\phi \in \pi_{2}\left(\theta_{a}, \theta_{a}\right)$ denotes the homotopy types of the two disks connecting $\theta_{a}$ to itself with boundary on $\beta \amalg \gamma, n_{w_{1}}(\phi)=1$ and $n_{w_{2}}(\phi)=0$. Similarly, $\psi \in \pi_{2}\left(\theta_{a}, \theta_{a}\right)$ denotes the homotopy types of the two disks connecting $\theta_{a}$ to itself with boundary on $\beta \amalg \gamma, n_{w_{1}}(\phi)=0$ and $n_{w_{2}}(\phi)=1$. Moreover, $t_{1, i}$ and $t_{2, i}$ are arbitrary point on $[0,1] \times \mathbb{R}$. It is implied by [AE15, Lemma 7.3] that the total number of points (counted with sign) in this latter end of the moduli space is 1. Consequently, $n_{p_{1}^{1}, p_{2}^{1}}\left(\Delta_{k_{1}, k_{2}}\right)=1$ and the proof is complete.

5.5. Invariance of the cobordism map for one-handles. Let $\mathcal{T}=[M, T, \mathfrak{u}, \mathfrak{s}]$ be an $\mathbb{A}$-tangle and $\mathbb{S} \subset M \backslash T$ be a framed 0-sphere. As before, $\mathcal{C}=\mathcal{C}(\mathbb{S})$ denotes the parametrized $\mathbb{A}$-cobordism from $\mathcal{T}$ to $\mathcal{T}^{\prime}=\mathcal{T}(\mathbb{S})$, obtained by attaching a 1-handle to $\mathcal{T} \times[0,1]$ along $\mathbb{S} \times\{1\}$.

Theorem 5.4. For any $\mathbb{A}$-module $\mathbb{M}$, the chain map $f_{\mathbb{S}}$ from Proposition 5.2 induces a homomorphism

$$
\mathfrak{f}_{\mathcal{C}}^{\mathbb{M}}: \operatorname{HF}^{\mathbb{M}}(\mathcal{T}) \longrightarrow \operatorname{HF}^{\mathbb{M}}\left(\mathcal{T}^{\prime}\right)
$$

Proof. Any Heegaard surface for $\mathcal{T}$ may be modified to intersect each component of $\mathbb{S}$ in a disk, by an isotopy supported in a neighborhood of two arcs in $M$. So we may just consider Heegaard diagrams for $\mathcal{T}$ that the underlying Heegaard surface satisfies in this property. From any Heegaard diagram $\mathcal{H}$ for $\mathcal{T}$ with such an underlying Heegaard surface $\Sigma$, one may construct a Heegaard diagram $\mathcal{H}^{\prime}$ for $\mathcal{T}^{\prime}$ as described in Section 5.1. Any choice of a path $J$ of almost complex structures for the Heegaard surface $\Sigma$ of $\mathcal{T}$ gives a degenerate path of almost complex structures corresponding to the join of $\Sigma$ and the standard model $\bar{S}$ at $w_{1}$ and $w_{2}$. This path may be perturbed to a path $J^{\prime}$ of almost complex structures corresponding to $\Sigma^{\prime}$, the Heegaard surface for $\mathcal{T}^{\prime}$. Let $J_{1}$ and $J_{2}$ be paths of almost complex structures for $\Sigma$ and $J_{1}^{\prime}$ and $J_{2}^{\prime}$ be the corresponding paths for $\Sigma^{\prime}$. It 
follows from standard techniques (involving a path connecting different choices of paths of almost complex structures) that

$$
f_{\mathbb{S}} \circ \Phi_{J_{1} \rightarrow J_{2}}=\Phi_{J_{1}^{\prime} \rightarrow J_{2}^{\prime}} \circ f_{\mathbb{S}}
$$

Let $\mathcal{H}_{0}$ and $\mathcal{H}_{1}$ be Heegaard diagrams for $\mathcal{T}$ such that $\mathcal{H}_{1}$ is obtained from $\mathcal{H}_{0}$ by one Heegaard move (i.e. isotopy on an $\alpha$ or $\beta$ curve, handleslide on an $\alpha$ or $\beta$ curve, stabilization or destabilization) denoted by $e$. We need to prove that the diagram

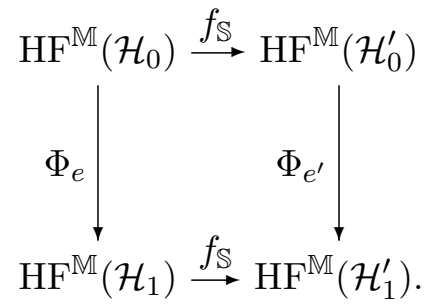

is commutative. Here, $\Phi_{e}$ is the isomorphism associated with the Heegaard move, and $\mathcal{H}_{i}^{\prime}$ is the Heegaard diagram for $\mathcal{T}^{\prime}$ obtained from $\mathcal{H}_{i}$ after joining it with $\mathcal{H}_{S}=\left(\mathbb{P}^{1}, \alpha, \beta, w_{1}, w_{2}\right)$ at $w_{1}$ and $w_{2}$. Furthermore, $\mathcal{H}_{1}^{\prime}$ is obtained from $\mathcal{H}_{0}^{\prime}$ by a Heegaard move, denoted by $e^{\prime}$.

When $e$ is a stabilization or destabilization, the proof is straightforward, and the above diagram is in fact commutative in the level of chain complexes if the almost complex structure is chosen correctly. The remaining cases are thus the cases where $e$ is an isotopy (which may pass over either of $w_{1}$ and $w_{2}$ ), or a handle slide.

We present the argument in the case where $e$ is a $\beta$-isotopy or a handle-slide on $\boldsymbol{\beta}$. Let $\boldsymbol{\gamma}$ be a collection of curves which is obtained from $\boldsymbol{\beta}$ by applying the Heegaard move $e$, followed by small Hamiltonian isotopies. Choose a small isotope $\gamma$ of $\beta$ as well. Let $\gamma^{\prime}=\gamma \amalg\{\gamma\}$. There is a generator associated with the Heegaard diagram $(\Sigma, \boldsymbol{\beta}, \boldsymbol{\gamma}, \mathfrak{u}, \mathfrak{s})$, denoted by $\Theta$, which represents the homology class $\Theta_{\beta \gamma}$. Furthermore, $\Theta^{\prime}=\Theta \times\left\{\theta_{\beta \gamma}\right\}$ generates the top homology class $\Theta_{\beta^{\prime} \gamma^{\prime}}$ in $\operatorname{HF}\left(\Sigma^{\prime}, \beta^{\prime}, \gamma^{\prime}, \mathfrak{u}^{\prime}, \mathfrak{s}^{\prime}\right)$.

The Heegaard triple $(\Sigma, \boldsymbol{\alpha}, \boldsymbol{\beta}, \boldsymbol{\gamma}, \mathfrak{u}, \mathfrak{s})$ determines a holomorphic triangle map, and together with the generator $\Theta$, this gives a chain map

$$
f=f_{\alpha \beta \gamma}: \mathrm{CF}(\Sigma, \boldsymbol{\alpha}, \boldsymbol{\beta}, \mathfrak{u}, \mathfrak{s}) \longrightarrow \mathrm{CF}(\Sigma, \boldsymbol{\alpha}, \gamma, \mathfrak{u}, \mathfrak{s}) .
$$

Similarly, the Heegaard triple $\left(\Sigma^{\prime}, \boldsymbol{\alpha}^{\prime}, \boldsymbol{\beta}^{\prime}, \boldsymbol{\gamma}^{\prime}, \mathfrak{u}^{\prime}, \mathfrak{s}^{\prime}\right)$ and the generator $\Theta^{\prime}$ determine a chain map

$$
f^{\prime}=f_{\alpha^{\prime} \beta^{\prime} \gamma^{\prime}}: \mathrm{CF}\left(\Sigma^{\prime}, \boldsymbol{\alpha}^{\prime}, \boldsymbol{\beta}^{\prime}, \mathfrak{u}^{\prime}, \mathfrak{s}^{\prime}\right) \longrightarrow \mathrm{CF}\left(\Sigma^{\prime}, \boldsymbol{\alpha}^{\prime}, \gamma^{\prime}, \mathfrak{u}^{\prime}, \mathfrak{s}^{\prime}\right) .
$$

To complete the proof, it is enough to show that $f^{\prime} \circ f_{\mathbb{S}}=f_{\mathbb{S}} \circ f$.

For this purpose, fix the generator $\mathbf{x}$ of $\mathrm{CF}(\Sigma, \boldsymbol{\alpha}, \boldsymbol{\beta}, \mathfrak{u}, \mathfrak{s})$ and suppose

$$
\Delta^{\prime} \in \pi_{2}\left(\mathbf{x} \times \theta_{\alpha \beta}, \Theta \times \theta_{\beta \gamma}, \mathbf{y} \times \theta\right)
$$

is a triangle class contributing to $f^{\prime} \circ f_{\mathbb{S}}$, where $\theta$ is one of the two intersection points in $\alpha \cap \gamma . \Delta^{\prime}$ gives the triangle classes

$$
\Delta \in \pi_{2}(\mathbf{x}, \Theta, \mathbf{y}) \quad \text { and } \quad \Delta_{S} \in \pi_{2}\left(\theta_{\alpha \beta}, \theta_{\beta \gamma}, \theta\right)
$$

which support holomorphic representatives. Moreover we have

$$
n_{w_{i}}(\Delta)=n_{w_{i}}\left(\Delta_{S}\right) \text { for } i=1,2 \quad \text { and } \quad 0=\mu\left(\Delta^{\prime}\right)=\mu(\Delta)+\mu\left(\Delta_{S}\right)-2 n_{w_{1}}\left(\Delta_{S}\right)-2 n_{w_{2}}\left(\Delta_{S}\right) .
$$

Since $\mu\left(\Delta_{S}\right)=|\theta|+2 n_{w_{1}}\left(\Delta_{S}\right)+2 n_{w_{2}}\left(\Delta_{S}\right)$ we find $\mu\left(\Delta^{\prime}\right)=\mu(\Delta)+|\theta|$. From here, we conclude that $\theta$ is the top generator $\theta_{\alpha \gamma}$ and that $\mu(\Delta)=0$.

Let $k_{i}=n_{w_{i}}\left(\Delta_{S}\right)$ for $i=1,2$ and consider the evaluation maps

$$
\begin{aligned}
& \rho_{\Sigma}=\rho_{w_{1}} \times \rho_{w_{2}}: \mathcal{M}(\Delta) \rightarrow \operatorname{Sym}^{k_{1}}(\mathbb{D}) \times \operatorname{Sym}^{k_{2}}(\mathbb{D}) \text { and } \\
& \rho_{S}=\rho_{w_{1}} \times \rho_{w_{2}}: \mathcal{M}\left(\Delta_{S}\right) \rightarrow \operatorname{Sym}^{k_{1}}(\mathbb{D}) \times \operatorname{Sym}^{k_{2}}(\mathbb{D}) .
\end{aligned}
$$

If $u^{\prime} \in \mathcal{M}\left(\Delta^{\prime}\right)$ corresponds to the degeneration $u \star u_{S}$ with $u \in \mathcal{M}(\Delta)$ and $u_{S} \in \mathcal{M}\left(\Delta_{S}\right)$, we find

$$
\rho_{\Sigma}(u)=\rho_{S}\left(u_{S}\right) \in \operatorname{Sym}^{k_{1}}(\mathbb{D}) \times \operatorname{Sym}^{k_{2}}(\mathbb{D}) .
$$


There are several classes $\Delta_{S}$ with the property that $n_{w_{i}}\left(\Delta_{S}\right)=k_{i}$. Let $\Delta_{k_{1}, k_{2}}$ denote the set of all the above classes. We thus obtain a map

$$
\imath_{\Delta^{\prime}}: \mathcal{M}\left(\Delta^{\prime}\right) \longrightarrow \mathcal{M}(\Delta) \times_{\operatorname{Sym}^{k_{1}}(\mathbb{D}) \times \operatorname{Sym}^{k_{2}(\mathbb{D})}} \mathcal{M}\left(\Delta_{k_{1}, k_{2}}\right) .
$$

By the argument of Proposition 5.1. if the neck is sufficiently stretched and the paths of almost complex structures are generic $\imath_{\Delta^{\prime}}$ is a bijection, reducing the proof to the second claim in Lemma 5.3. This completes the proof of Theorem 5.4.

5.6. Three-handles. Let $\mathcal{T}=[M, T, \mathfrak{u}, \mathfrak{s}]$ be an $\mathbb{A}$-tangle as before, and $\mathbb{S} \subset M \backslash T$ be a framed 2 -sphere. Further, assume that $\left\langle c_{1}(\mathfrak{s}),[a(\mathbb{S})]\right\rangle=0$, where $a(\mathbb{S})$ is the attaching sphere of $\mathbb{S}$. Then, $\mathbb{S}$ specifies an $\mathbb{A}$-cobordism

$$
\mathcal{C}=\mathcal{C}(\mathbb{S})=\left[W(\mathbb{S}), F(\mathbb{S})=T \times[0,1], \mathfrak{u}_{F}: \pi_{0}(T)=\pi_{0}(F(\mathbb{S})) \rightarrow \mathbb{A}, \mathfrak{t}_{\mathfrak{s}}\right],
$$

where $W(\mathbb{S})$ is obtained from $M \times[0,1]$ by attaching a 3-handle along the framed sphere $\mathbb{S} \times\{1\} \subset$ $M \times\{1\}$. The $\operatorname{Spin}^{c}$ class $\mathfrak{t}=\mathfrak{t}_{\mathfrak{s}}$ is the $\operatorname{Spin}^{c}$ structure on $W=W(\mathbb{S})$ determined by $\mathfrak{s}$ i.e. $\left.\mathfrak{t}\right|_{M}=\mathfrak{s}$. The cobordism $\mathcal{C}(\mathbb{S})$ connects $\mathcal{T}$ to

$$
\mathcal{T}^{\prime}=\mathcal{T}(\mathbb{S})=\left[M^{\prime}=M(\mathbb{S}), T^{\prime}=T \times\{1\}, \mathfrak{u}^{\prime}: \pi_{0}\left(T^{\prime}\right)=\pi_{0}(T) \rightarrow \mathbb{A}, \mathfrak{s}^{\prime}=\left.\mathfrak{t}\right|_{M^{\prime}}\right] .
$$

If we reverse the cobordism $\mathcal{C}(\mathbb{S})$ we obtain an $\mathbb{A}$-cobordism from $\mathcal{T}^{\prime}$ to $\mathcal{T}$ which corresponds to attaching a 1-handle.

Consider a Heegaard surface $\Sigma$ for $M$ that cuts the framed sphere $\mathbb{S}$ in a cylinder $S \subset \Sigma$ with boundary components $C_{1}$ and $C_{2}$. A Heegaard surface $\Sigma^{\prime}$ for $M^{\prime}$ is then obtained from $\Sigma$ by removing $S$, and gluing a pair of disks with centers $w_{1}$ and $w_{2}$ to $C_{1}$ and $C_{2}$, respectively. Let $\mathcal{H}^{\prime}=\left(\Sigma^{\prime}, \boldsymbol{\alpha}^{\prime}, \boldsymbol{\beta}^{\prime}, \mathfrak{u}^{\prime}: \mathbf{z} \rightarrow \mathbb{A}, \mathfrak{s}^{\prime}\right)$ be a Heegaard diagram for $\mathcal{T}^{\prime}$. Consider a pair of Hamiltonian isotopic curves $\alpha$ and $\beta$ on $S$ that are both isotopic to the core of the cylinder and cut each other transversely in $\theta_{\alpha \beta}$ and $\theta_{\beta \alpha}$. Then,

$$
\mathcal{H}=\left(\Sigma, \boldsymbol{\alpha}=\boldsymbol{\alpha}^{\prime} \cup\{\alpha\}, \boldsymbol{\beta}=\boldsymbol{\beta}^{\prime} \cup\{\beta\}, \mathfrak{u}: \mathbf{z} \rightarrow \mathbb{A}, \mathfrak{s}\right)
$$

is a Heegaard diagram for $\mathcal{T}$, provided that we use the stronger form of admissibility for $\mathcal{H}^{\prime}$, as discussed in [AE15, Remark 4.6].

Choose a generic path $J^{\prime}$ of almost complex structures associated with the surface $\Sigma^{\prime}$. On the sphere $\bar{S}$, obtained by attaching a pair of disks with centers $w_{1}$ and $w_{2}$ to $S$, consider a complex structure $J_{S}$, so that $\left(\bar{S}, w_{1}, w_{2}\right)$ is identified with $\left(\mathbb{P}^{1}, 0, \infty\right)$. Then, let $J$ be a generic path of almost complex structures on $\Sigma$ sufficiently close to the join of $J$ and $J_{S}$. The chain map

$$
f_{\mathbb{S}}: \mathrm{CF}_{J}(\mathcal{H}) \longrightarrow \mathrm{CF}_{J^{\prime}}\left(\mathcal{H}^{\prime}\right) \text {. }
$$

is defined as follows. For a generator

$$
\mathbf{x}=\mathbf{x}^{\prime} \times \theta \in \mathbb{T}_{\alpha} \cap \mathbb{T}_{\beta}=\left(\mathbb{T}_{\alpha^{\prime}} \cap \mathbb{T}_{\beta^{\prime}}\right) \times(\alpha \cap \beta)
$$

let

$$
f_{\mathbb{S}}(\mathbf{x})= \begin{cases}\mathbf{x}^{\prime} & \text { if } \theta=\theta_{\beta \alpha} \\ 0 & \text { otherwise }\end{cases}
$$

An argument, which is basically the dual of the argument used for 1-handles implies the following theorem.

Theorem 5.5. With the above notation fixed, $f_{\mathbb{S}}$ is a chain map, and for any $\mathbb{A}$-module $\mathbb{M}$, it induces a natural homomorphism

$$
\mathfrak{f}_{\mathbb{S}}^{\mathbb{M}}=\mathfrak{f}_{\mathcal{C}}^{\mathbb{M}}: \operatorname{HF}^{\mathbb{M}}(\mathcal{T}) \longrightarrow \operatorname{HF}^{\mathbb{M}}\left(\mathcal{T}^{\prime}\right)
$$




\section{Framed ARCS, FRAMED KNOTS AND COBORDism MAPS}

6.1. Special tangles corresponding to cobordisms. Assume $\mathcal{C}=\left[W, F, \mathfrak{t}, \mathfrak{u}_{F}\right]$ is an $\mathbb{A}$-cobordism from the $\mathbb{A}$-tangle $\mathcal{T}=[M, T, \mathfrak{s}, \mathfrak{u}]$ to another $\mathbb{A}$-tangle $\mathcal{T}^{\prime}=\left[M^{\prime}, T^{\prime}, \mathfrak{s}^{\prime}, \mathfrak{u}^{\prime}\right]$. In this subsection we introduce an $\mathbb{A}$-tangle $\mathcal{T}_{F}$ associated with $\mathcal{C}$ playing the role of $\#^{k} S^{1} \times S^{2}$ in defining the chain maps in Heegaard Floer theory. The important feature of $\mathcal{T}_{F}$ is the existence of a distinguished generator $\Theta_{F} \in \operatorname{HF}\left(\mathcal{T}_{F}\right)$, which plays the role of the top generator in $\mathrm{HF}^{-}\left(\#^{k} S^{1} \times S^{2}\right)$.

Denote the stable cobordism $(W, F)$ by $\mathcal{W}$. Recall that the positive boundary of $\mathcal{W}$, denote by $\partial^{+} \mathcal{W}=\left(M^{+}, T^{+}\right)$, is identified with the product tangle

$$
\left(M^{+}, T^{+}\right)=\left(\partial^{+} M \times[0,1], \partial^{+} T \times[0,1]\right) .
$$

Thus, denote

$$
\left(\partial^{+} M^{+}, \partial^{+} T^{+}\right)=\left(\partial^{+} M \times\{1\}, \partial^{+} T \times\{1\}\right) \text { and }\left(\partial^{-} M^{+}, \partial^{-} T^{+}\right)=\left(\partial^{+} M \times\{0\}, \partial^{+} T \times\{0\}\right) .
$$

Let $J \subset F$ be a properly embedded, simple arc such that $\partial J \subset T^{+}$. Associated with $J$ we define a pair $\left(M_{J}, T_{J}\right)$ by doing surgery on $\left(M^{+}, T^{+}\right)$at points $J \cap T^{+}$with the framing induced by $F$. More precisely, let $\operatorname{nd}(J) \subset W$ be a small tubular neighborhood of $J$ in $W$. The intersection $F \cap \partial \operatorname{nd}(J)$ induces a framing on $\partial \operatorname{nd}(J)$. Using this framing, we define

$$
M_{J}=\left(M^{+} \backslash M^{+} \cap \operatorname{nd}(J)\right) \cup \partial \operatorname{nd}(J) \quad \text { and } \quad T_{J}=\partial(F \backslash \operatorname{nd}(J)) \backslash\left(\partial F \cap\left(\partial W \backslash M^{+}\right)\right) .
$$

If the end points of $J$ are on distinct connected components of $T^{+}$then $\left(M_{J}, T_{J}\right)$ is a balanced tangle. In this case, consider a small product neighborhood of $M^{+}$, and an identification of it with $M^{+} \times[0, \epsilon]$, such that $F \cap\left(M^{+} \times[0, \epsilon]\right)=T^{+} \times[0, \epsilon]$. Denote the 4-manifold obtained from attaching the one-handle nd $(J)$ to $M^{+} \times[0, \epsilon]$ by $W_{J}$. Let $F_{J}=F \cap W_{J}$ and note that $\mathcal{W}_{J}=\left(W_{J}, F_{J}\right)$ is a cobordism from $\left(M^{+}, T^{+}\right)$to $\left(M_{J}, T_{J}\right)$.

Definition 6.1. A set $J=\left\{J_{1}, \ldots, J_{n}\right\}$ of pairwise disjoint, properly embedded, simple arcs on $F$ satisfying $\partial J_{i} \subset T^{+}$for $i=1, \ldots, n$ is called a spanning set if each connected component of $F \backslash\left(\coprod_{i=1}^{n} J_{i}\right)$ is a disc and contains exactly one connected component of $T$ and one connected component of $T^{\prime}$.

Consider a spanning set $J=\left\{J_{1}, \ldots, J_{n}\right\}$ of arcs on $F$. After doing surgery on $\left(M^{+}, T^{+}\right)$along the elements of $J$, we get a balanced tangle, which is denoted by $\left(M_{J}, T_{J}\right)$. In particular, if $J$ is a single arc, $\left(M_{\{J\}}, T_{\{J\}}\right)=\left(M_{J}, T_{J}\right)$. The diffeomorphism type of $\left(M_{J}, T_{J}\right)$ does not change under an arc slide move, i.e. sliding one foot of an $\operatorname{arc} J_{i}$ over another $\operatorname{arc} J_{j}$, while fixing the rest of the arcs.

Lemma 6.1. The diffeomorphism type of the tangle $\left(M_{J}, T_{J}\right)$ is independent of the choice of $J$.

Proof. Associated with any spanning set $J$ of $\operatorname{arcs}$ on $F$, we may define a Morse function $g: F \rightarrow[0,1]$ and a gradient like vector field $\xi$, such that

(1) $\left.g\right|_{F \cap \partial^{-} W} \equiv 0,\left.g\right|_{F \cap \partial^{+} W} \equiv 1$ and $g$ has no critical point in a neighborhood of $\partial F$.

(2) All critical points of $g$ have index one.

(3) The arcs in $J$ are the unstable manifolds of the critical points of $g$.

It follows from [GK15, Theorem 4.5] that any two Morse data $(g, \xi)$ and $\left(g^{\prime}, \xi^{\prime}\right)$ as above, can be connected by a sequence of critical point switches and isotopies of the gradient-like vector fields. Thus, the corresponding spanning sets can be connected by a sequence of arc slides. So, $\left(M_{J}, T_{J}\right)$ does not depend on the choice of $J$.

We may thus denote the balanced tangle $\left(M_{J}, T_{J}\right)$ by $\left(M_{F}, T_{F}\right)$ and the cobordism $\mathcal{W}_{J}$ from $\left(M^{+}, T^{+}\right)$to $\left(M_{F}, T_{F}\right)$ by $\mathcal{W}_{F}$.

Definition 6.2. A spanning set $J=\left\{J_{1}, \ldots, J_{n}\right\}$ of arcs is called ordered, if

(1) endpoints of each $J_{i}$ lie on distinct components of $T^{+}$, 
(2) if for some $j<k$ an endpoint $e_{j}$ of $J_{j}$ and an endpoint $e_{k}$ of $J_{k}$ are on the same component of $T^{+}$, then $\pi\left(e_{j}\right)>\pi\left(e_{k}\right)$. Here, $\pi: T^{+}=\amalg_{i=1}^{\kappa}\left(\left\{p_{i}\right\} \times[0,1]\right) \rightarrow[0,1]$ denotes the projection on $[0,1]$.

If a spanning set $J=\left\{J_{1}, \ldots, J_{n}\right\}$ of arcs is ordered, doing surgery along $J_{1}, \ldots, J_{i}$ on $\left(M^{+}, T^{+}\right)$ would result in a balanced tangle, for any $1 \leq i \leq n$.

Lemma 6.2. For any stable cobordism $(W, F)$, there is an ordered spanning set of arcs on $F$.

Proof. The proof is straightforward.

The $\mathbb{A}$-coloring $\mathfrak{u}_{F}$ induces an $\mathbb{A}$-coloring $\mathfrak{u}_{F} \circ \imath$ on $\left(M_{F}, T_{F}\right)$, where $\imath: \pi_{0}\left(T_{F}\right) \rightarrow \pi_{0}(F)$ is defined by inclusion. Abusing the notation we denote this coloring by $\mathfrak{u}_{F}$. Let $H_{i} \in \mathrm{H}_{2}\left(M_{F}, \mathbb{Z}\right)$ be the homology class represented by the belt sphere of the attached 1-handle corresponding to $J_{i}$. Moreover, associated with every component $\partial_{k}^{+} M$ of $\partial^{+} M$, with $k=1, \ldots, \ell$, we obtain a homology class $H_{k}^{\prime} \in \mathrm{H}_{2}\left(M_{F}, \mathbb{Z}\right)$. Consider the $\operatorname{Spin}^{c}$ class $\mathfrak{s}_{0} \in \operatorname{Spin}^{c}\left(M_{F}\right)$ such that $\left\langle c_{1}\left(\mathfrak{s}_{0}\right), H_{i}\right\rangle=$ $\left\langle c_{1}\left(\mathfrak{s}_{0}\right), H_{k}^{\prime}\right\rangle=0$ for every $1 \leq i \leq m$ and every $1 \leq k \leq \ell$. We then define $\mathcal{T}_{F}=\left[M_{F}, T_{F}, \mathfrak{s}_{0}, \mathfrak{u}_{F}\right]$.

Similar to $\mathcal{T}_{F}$, we define another tangle $\mathcal{T}_{F}^{b}$ as follows. Let us assume that $\partial^{+} T=\left\{p_{1}, \ldots, p_{\kappa}\right\} \subset$ $\partial^{+} M$ and correspondingly, $T^{+}$is a union of components

$$
T^{+}=\coprod_{i=1}^{\kappa} T_{i}^{+}=\coprod_{i=1}^{\kappa}\left(\left\{p_{i}\right\} \times[0,1]\right) \subset M^{+} .
$$

For every $1 \leq i \leq \kappa$ consider a point $\bar{p}_{i} \in \partial^{+} M$ close to $p_{i}$ and let $\bar{T}_{i}^{+}=\bar{p}_{i} \times[0,1]$ and $\bar{T}^{+}:=\amalg_{i=1}^{\kappa} \bar{T}_{i}^{+}$ in $M^{+}$. Setting $T_{F}^{b}=T_{F} \amalg \bar{T}^{+}$one gets a balanced tangle $\left(M_{F}, T_{F}^{b}\right)$.

Suppose that $F=\coprod_{i=1}^{m} F_{i}$ and $\partial^{+} M=\amalg_{k=1}^{\ell} \partial_{k}^{+} M$. Associated to each component $\partial_{k}^{+} M$ of $\partial^{+} M$, we define a monomial

$$
\mathfrak{w}_{k}=\prod_{p_{j} \in \partial_{k}^{+} M}\left(\mathfrak{u}\left(p_{j}\right) \mathfrak{v}_{j}\right) \in \mathbb{F}\left[\mathfrak{u}_{1}, \ldots, \mathfrak{u}_{m}, \mathfrak{v}_{1}, \ldots, \mathfrak{v}_{\kappa}\right]
$$

where $\mathfrak{u}\left(p_{j}\right)=\mathfrak{u}_{i}$ if $p_{j} \in F_{i}$. Denote the genus of $\partial_{k}^{+} M$ by $g_{k}$. With this notation fixed, let

$$
\mathbb{A}_{F}^{b}:=\frac{\mathbb{F}\left[\mathfrak{u}_{1}, \ldots, \mathfrak{u}_{m}, \mathfrak{v}_{1}, \ldots, \mathfrak{v}_{\kappa}\right]}{\left\langle\mathfrak{w}_{k} \mid g_{k}>0, k=1, \ldots, \ell\right\rangle} .
$$

The map $\mathfrak{u}_{F}^{b}: \pi_{0}\left(T_{F}^{b}\right) \rightarrow \mathbb{A}_{F}^{b}$ defined by

$$
\begin{cases}\mathfrak{u}_{F}^{b}\left(\bar{T}_{j}^{+}\right)=\mathfrak{v}_{j} & 1 \leq j \leq \kappa \\ \mathfrak{u}_{F}^{b}\left(T_{F}^{j}\right)=\mathfrak{u}\left(p_{j}\right) & 1 \leq j \leq \kappa,\end{cases}
$$

is an $\mathbb{A}_{F}^{b}$-coloring of $\left(M_{F}, T_{F}^{b}\right)$. Here, $T_{F}^{j}$ denotes the component of $T_{F}$ that intersects $\partial^{+} M$ in $p_{j}$. As a result, we get an $\mathbb{A}_{F}^{b}$-tangle $\mathcal{T}^{b}=\left[M_{F}, T_{F}^{b}, \mathfrak{s}_{0}, \mathfrak{u}_{F}^{b}\right]$.

Remark 6.3. Let $\mathbb{A}$ denotes the $\mathbb{Z}$-algebra associated with the sutured manifold corresponding to $\left(M_{F}, T_{F}^{b}\right)$, see Section 2.1. Then $\mathbb{A}_{F}^{b}$ is the quotient of $\mathbb{A} \otimes \mathbb{F}$ by the ideal generated by the binomials $\mathfrak{u}_{i}-\mathfrak{u}_{j}$ for all $i$ and $j$, so that $T_{F}^{i}$ and $T_{F}^{j}$ lie on the boundary of the same component of $F$.

The algebra $\mathbb{A}$ has a natural $\mathbb{A}_{F}^{b}$-module structure given by the homomorphism $\phi: \mathbb{A}_{F}^{b} \rightarrow \mathbb{A}$ defined as

$$
\begin{cases}\phi\left(\mathfrak{u}_{i}\right)=\mathfrak{u}_{F}\left(F_{i}\right) & 1 \leq i \leq m \\ \phi\left(\mathfrak{v}_{j}\right)=1 & 1 \leq j \leq \kappa .\end{cases}
$$

Further, considering this module structure on $\mathbb{A}$ it is straightforward that

$$
\operatorname{HF}^{\mathbb{A}}\left(\mathcal{T}^{b}\right)=\operatorname{HF}\left(\mathcal{T}_{F}\right) .
$$


6.2. The distinguished generator. For every $1 \leq i \leq \kappa$, consider a product disk $\left(D_{i}, \partial D_{i}\right)$ in the balanced tangle $\left(M^{+}, T^{+} \amalg \bar{T}^{+}\right)$such that

$$
\bar{T}_{i}^{+} \amalg-T_{i}^{+} \subset \partial D_{i} \quad \text { and } \quad \partial D_{i} \backslash\left(\bar{T}_{i}^{+} \amalg-T_{i}^{+}\right) \subset \partial M^{+} .
$$

Abusing the notation, we denote the intersection of $D_{i}$ with $M_{F}$ by $D_{i}$ as well. Let $D=\amalg_{i=1}^{\kappa} D_{i}$. For every $1 \leq j \leq n$, we may attach an oriented one handle $D_{j}^{\prime}$ (i.e. a band) to $D$ such that $D_{j}^{\prime} \subset M_{F}$ is embedded in the one handle associated with $J_{j}$ and

$$
\partial D_{j}^{\prime} \backslash\left(\partial D_{j}^{\prime} \cap D\right) \subset F
$$

Denote the resulting embedded, oriented surface in $\left(M_{F}, T_{F}^{b}\right)$ by $F^{\prime}$. Note that

$$
\left(F^{\prime}, \partial F^{\prime}\right) \subset\left(M_{F}, \partial M_{F} \cup T_{F}^{b}\right)
$$

and that this pair is uniquely determined up to isotopy.

Let $\left(X_{F}, \tau_{F}^{b}\right)$ be the balanced sutured manifold associated with the tangle $\left(M_{F}, T_{F}^{b}\right)$. The surface $F^{\prime} \cap X_{F} \subset X_{F}$ is a decomposing surface for $\left(X_{F}, \tau_{F}^{b}\right)$, in the sense of [Juh08, Definition 2.7]. It is straightforward to check that this surface decomposes $\left(X_{F}, \tau_{F}^{b}\right)$ into a product sutured manifold. Thus, there exists a unique relative $\operatorname{Spin}^{c}$ structure $\underline{\mathfrak{s}}_{F^{\prime}} \in \operatorname{Spin}^{c}\left(X_{F}, \tau_{F}^{b}\right)$ which is outer with respect to $F^{\prime}$. Recall that $\underline{\mathfrak{s}} \in \operatorname{Spin}^{c}\left(X_{F}, \tau_{F}^{b}\right)$ is called outer with respect to $F^{\prime}$, if it is represented by a unit vector field $v$ on $X_{F}$ such that $v_{p} \neq-\left(\nu_{F^{\prime}}\right)_{p}$ for every $p \in F^{\prime}$. Here $\nu_{F^{\prime}}$ is the unit normal vector field of $F^{\prime}$ with respect to some Riemannian metric on $X_{F}$, See [Juh08, Definition 1.1]. Note that $\left[\underline{\mathfrak{s}}_{F^{\prime}}\right]=\mathfrak{s}_{0}$.

If the components $T_{F}^{j_{1}}$ and $T_{F}^{j_{2}}$ of $T_{F}$ lie on the same component $F_{i}$ of $F$, then

$$
\left[\tau_{F}^{j_{1}}\right]=\left[\tau_{F}^{j_{2}}\right] \in \mathrm{H}_{1}\left(X_{F}, \mathbb{Z}\right)
$$

where $\tau_{F}^{j}$ denotes the suture associated with $T_{F}^{j}$. Thus, for any component $F_{i}$ of $F$, there is a well-defined homology class $h_{i}=\left[\tau_{F}^{j}\right] \in \mathrm{H}_{1}\left(X_{F}, \mathbb{Z}\right)$ where $T_{F}^{j} \subset F_{i}$. As a result, the algebra $\mathbb{A}_{F}^{b}$ admits a filtration by $\mathbb{H}=\mathrm{H}^{2}\left(X_{F}, \partial X_{F}, \mathbb{Z}\right)$, which is defined by

$$
\begin{aligned}
& \chi: G\left(\mathbb{A}_{F}^{b}\right) \rightarrow \mathbb{H}=\mathrm{H}^{2}\left(X_{F}, \partial X_{F}, \mathbb{Z}\right) \\
& \chi\left(\prod_{i=1}^{m} \mathfrak{u}_{i}^{a_{i}} \prod_{j=1}^{\kappa} \mathfrak{v}_{j}^{b_{j}}\right):=\sum_{i=1}^{m} a_{i} \mathrm{PD}\left(h_{i}\right)+\sum_{j=1}^{\kappa} b_{j} \operatorname{PD}\left(\bar{\tau}_{j}^{+}\right)
\end{aligned}
$$

where $\bar{\tau}_{j}^{+}$is the suture corresponding to $\bar{T}_{j}^{+} \subset T_{F}^{b}$. Therefore, for every $\mathfrak{s} \in \operatorname{Spin}^{c}\left(M_{F}\right)$

$$
\mathrm{CF}\left(M_{F}, T_{F}^{b}, \mathfrak{u}_{F}^{b}, \mathfrak{s}\right)
$$

may be decomposed into sub-complexes associated with relative $\operatorname{Spin}^{c}$ structures. More precisely, let $\mathcal{H}=\left(\Sigma, \boldsymbol{\alpha}, \boldsymbol{\beta}, \mathfrak{u}_{F}^{b}: \mathbf{z} \amalg \overline{\mathbf{z}} \rightarrow \mathbb{A}_{F}^{b}, \mathfrak{s}_{0}\right)$ be an $\mathbb{A}_{F}^{b}$-diagram for $\mathcal{T}_{F}^{b}$, where $\mathbf{z}=\left\{z_{1}, \ldots, z_{\kappa}\right\}$ and $\overline{\mathbf{z}}=\left\{\bar{z}_{1}, \ldots, \bar{z}_{\kappa}\right\}$, while $z_{i}=T_{F}^{i} \cap \Sigma$ and $\bar{z}_{i}=\bar{T}_{i}^{+} \cap \Sigma$. Then

$$
\mathrm{CF}\left(\Sigma, \boldsymbol{\alpha}, \boldsymbol{\beta}, \mathfrak{u}_{F}^{b}, \mathfrak{s}_{0}\right)=\bigoplus_{\underline{\mathfrak{s}} \in \mathfrak{s}_{0} \subset \operatorname{Spin}^{c}\left(M_{F}, T_{F}^{b}\right)} \mathrm{CF}\left(\Sigma, \boldsymbol{\alpha}, \boldsymbol{\beta}, \mathfrak{u}_{F}^{b}, \underline{\mathfrak{s}}\right)
$$

and $\operatorname{CF}\left(\Sigma, \boldsymbol{\alpha}, \boldsymbol{\beta}, \mathfrak{u}_{F}^{b}, \underline{\mathfrak{s}}\right)$ is generated by the elements $\mathfrak{u} . \mathbf{x}$, where $\mathfrak{u} \in G\left(\mathbb{A}_{F}^{b}\right)$ is a monomial in $\mathbb{A}_{F}^{b}$ and $\mathbf{x} \in \mathbb{T}_{\alpha} \cap \mathbb{T}_{\beta}$ is an intersection point satisfying

$$
\underline{\mathfrak{s}}(\mathfrak{u} \cdot \mathbf{X}):=\underline{\mathfrak{s}}(\mathbf{x})+\chi(\mathfrak{u})=\underline{\mathfrak{s}} .
$$

For a relative class $\underline{\mathfrak{s}} \in \mathfrak{s}_{0}$, let

$$
\mathfrak{u}=\prod_{i=1}^{m} \mathfrak{u}_{i}^{a_{i}} \prod_{j=1}^{\kappa} \mathfrak{v}_{j}^{b_{j}} \text { and } \mathfrak{u}^{\prime}=\prod_{i=1}^{m} \mathfrak{u}_{i}^{c_{i}} \prod_{j=1}^{\kappa} \mathfrak{v}_{j}^{d_{j}}
$$


be non-zero monomials in $G\left(\mathbb{A}_{F}^{b}\right)$ and $\mathbf{x}, \mathbf{y} \in \mathbb{T}_{\alpha} \cap \mathbb{T}_{\beta}$ be intersection points such that $\mathfrak{\mathfrak { s }}(\mathfrak{u} \cdot \mathbf{x})=$ $\underline{\mathfrak{s}}\left(\mathfrak{u}^{\prime} . \mathbf{y}\right)=\underline{\mathfrak{s}}$. Then, we say $\phi \in \pi_{2}(\mathbf{x}, \mathbf{y})$ connects $\boldsymbol{u} . \mathbf{x}$ to $\mathfrak{u}^{\prime} . \mathbf{y}$ if

$$
\begin{cases}a_{i}+\sum_{T_{F}^{j} \subset F_{i}} n_{z_{j}}(\phi)=c_{i} & 1 \leq i \leq m \\ b_{j}+n_{\bar{z}_{j}}(\phi)=d_{j} & 1 \leq j \leq \kappa .\end{cases}
$$

Moreover, we define the relative grading of $\mathfrak{u} . \mathbf{x}$ and $\mathfrak{u}^{\prime} . \mathbf{y}$ by

$$
\operatorname{gr}\left(\mathfrak{u} \cdot \mathbf{x}, \mathfrak{u}^{\prime} \cdot \mathbf{y}\right)=\mu(\phi)
$$

where $\phi \in \pi_{2}(\mathbf{x}, \mathbf{y})$ is a disk connecting $\mathfrak{u} . \mathbf{x}$ to $\mathfrak{u}^{\prime} \cdot \mathbf{y}$.

Lemma 6.4. The relative grading gr is well-defined and is independent of the choice of $\phi$. It induces a relative grading on the chain complex $\mathrm{CF}\left(M_{F}, T_{F}^{b}, \mathfrak{u}_{F}^{b}, \mathfrak{s}\right)$ and the differential lowers this grading by one.

Proof. Suppose $\phi, \phi^{\prime} \in \pi_{2}(\mathbf{x}, \mathbf{y})$ connect $\mathfrak{u} . \mathbf{x}$ to $\mathfrak{u}^{\prime} . \mathbf{y}$. Then, $\mathcal{D}=\mathcal{D}(\phi)-\mathcal{D}\left(\phi^{\prime}\right)$ is a periodic domain such that

$$
\sum_{T_{F}^{j} \subset F_{i}} n_{z_{j}}(\mathcal{D})=0 \quad \text { and } \quad n_{\bar{z}_{j}}(\mathcal{D})=0
$$

for any $i$ and $j$. Thus $\mathcal{D}=\sum_{i=1}^{n} m_{i} \mathcal{P}_{i}$ where $\mathcal{P}_{i}$ is the periodic domain associated with $H_{i}$. Since, $\left\langle c_{1}\left(\mathfrak{s}_{0}\right), H_{i}\right\rangle=0$ for any $i$,

$$
\mu(\phi)-\mu\left(\phi^{\prime}\right)=\left\langle c_{1}\left(\mathfrak{s}_{0}\right), H(\mathcal{D})\right\rangle=0 .
$$

Proposition 6.5. With respect to the relative grading defined above, the top-dimensional homology group in $\operatorname{HF}\left(M_{F}, T_{F}^{b}, \mathfrak{u}_{F}^{b}, \underline{\mathfrak{s}}_{F^{\prime}}\right)$ is isomorphic to $\mathbb{F}$. If $\Theta_{F}^{b}$ denotes the generator of this homology group, then for the homomorphism

$$
\phi: \operatorname{HF}\left(M_{F}, T_{F}^{b}, \mathfrak{u}_{F}^{b}, \underline{\mathfrak{s}}_{F^{\prime}}\right) \longrightarrow \mathrm{HF}^{\mathbb{F}}\left(M_{F}, T_{F}^{b}, \mathfrak{u}_{F}^{b}, \underline{\mathfrak{s}}_{F^{\prime}}\right)=\operatorname{SFH}\left(X_{F}, \tau_{F}^{b}, \underline{\mathfrak{s}}_{F^{\prime}}\right)=\mathbb{F},
$$

we have $\phi\left(\Theta_{F}^{b}\right)=1$. Here, $\mathbb{F}$ is an $\mathbb{A}_{F}^{b}$-module with trivial module structure and the homomorphism $\phi$ is induced from the surjection $\mathbb{A}_{F}^{b} \rightarrow \mathbb{F}$, which sends all variables to zero.

Proof. Let $\pi: T^{+}=\amalg_{i=1}^{\kappa}\left(\left\{p_{i}\right\} \times[0,1]\right) \rightarrow[0,1]$ denote the projection on [0,1]. Consider an ordered set of spanning arcs $J=\left\{J_{1}, \cdots, J_{n}\right\}$ on $F$. The result of surgery on $\left(M^{+}, T^{+} \amalg \bar{T}^{+}\right)$ along $J_{1}, \ldots, J_{i}$ is a balanced tangle for any $i$, denoted by $\left(M^{i}, T^{i} \amalg \bar{T}^{i}\right)$. Note that $\bar{T}^{i}=\bar{T}^{+}$for any $i$, and $\left(M^{n}, T^{n} \amalg \bar{T}^{n}\right)=\left(M_{F}, T_{F}^{b}\right)$.

Let $\mathfrak{s}_{0}^{i}$ denote the $\operatorname{Spin}^{c}$ structure on $M^{i}$ such that for any $1 \leq j \leq i$ we have $\left\langle c_{1}\left(\mathfrak{s}_{0}^{i}\right), H_{j}\right\rangle=0$ and for $1 \leq k \leq \ell$ we have $\left\langle c_{1}\left(\mathfrak{s}_{0}^{i}\right), H_{k}^{\prime}\right\rangle=0$. Thus, $\mathfrak{s}_{0}^{n}=\mathfrak{s}_{0}$. Assume that the components of $T^{i}=\amalg_{j=1}^{\kappa} T_{j}^{i}$ are labeled such that $T_{j}^{i} \cap \partial^{+} M=\left\{p_{j}\right\}$. Inductively, we define an $\mathbb{F}$-algebra $\mathbb{A}^{i}$ and a map $\mathfrak{u}^{i}: \pi_{0}\left(T^{i} \amalg \bar{T}^{i}\right) \rightarrow \mathbb{A}^{i}$, such that $\mathcal{T}^{i}=\left[M^{i}, T^{i} \amalg \bar{T}^{i}, \mathfrak{s}_{0}^{i}, \mathfrak{u}^{i}\right]$ becomes an $\mathbb{A}^{i}$-tangle. For $i=0$, we have $\left(M^{0}, T^{0} \amalg \bar{T}^{0}\right)=\left(M^{+}, T^{+} \amalg \bar{T}^{+}\right)$. Let

$$
\mathbb{A}^{0}:=\frac{\mathbb{Z}_{2}\left[\mathfrak{v}_{1}^{\prime}, \ldots, \mathfrak{v}_{\kappa}^{\prime}, \mathfrak{v}_{1}, \ldots, \mathfrak{v}_{\kappa}\right]}{\left\langle\mathfrak{u}^{k} \mid g_{k}>0\right\rangle}
$$

where $\mathfrak{u}^{k}=\prod_{p_{j} \in \partial_{k}^{+} M}\left(\mathfrak{v}_{j} \mathfrak{v}_{j}^{\prime}\right)$. An $\mathbb{A}^{0}$-coloring $\mathfrak{u}^{0}: \pi_{0}\left(T^{0} \amalg \bar{T}^{0}\right) \rightarrow \mathbb{A}^{0}$ is defined as

$$
\mathfrak{u}^{0}\left(T_{j}^{0}\right)=\mathfrak{v}_{j}^{\prime} \quad \text { and } \quad \mathfrak{u}^{0}\left(\bar{T}_{j}^{0}\right)=\mathfrak{v}_{j} \quad \text { for every } \quad 1 \leq j \leq \kappa .
$$

Then, if $J_{i}$ connects $T_{a}^{i-1}$ and $T_{b}^{i-1}$, we define

$$
\mathbb{A}^{i}:=\frac{\mathbb{A}^{i-1}}{\left\langle\mathfrak{u}^{i-1}\left(T_{a}^{i-1}\right)-\mathfrak{u}^{i-1}\left(T_{b}^{i-1}\right)\right\rangle}
$$


and $\mathfrak{u}^{i}: \pi_{0}\left(T^{i} \amalg \bar{T}^{i}\right) \rightarrow \mathbb{A}^{i}$ is the map induced by $\mathfrak{u}^{i-1}$. Note that $\mathbb{A}^{n}=\mathbb{A}_{F}^{b}$.

We may also construct an $\mathbb{A}^{i}$-diagram

$$
\mathcal{H}^{i}=\left(\Sigma^{i}, \boldsymbol{\alpha}^{i}, \boldsymbol{\beta}^{i}, \mathfrak{u}^{i}: \mathbf{z}^{i} \amalg \overline{\mathbf{z}}^{i} \rightarrow \mathbb{A}^{i}, \mathfrak{s}_{0}^{i}\right)
$$

for $\mathcal{T}^{i}$, inductively. For $i=0, \mathcal{T}^{0}$ is a product tangle so let $\Sigma^{0}=\partial^{+} M \times\{1 / 2\}, \boldsymbol{\alpha}^{0}=\boldsymbol{\beta}^{0}=\emptyset$, $\mathbf{z}=\left\{p_{1}, \ldots, p_{\kappa}\right\} \times\{1 / 2\}$ and $\overline{\mathbf{z}}=\left\{\bar{p}_{1}, \ldots, \bar{p}_{\kappa}\right\} \times\{1 / 2\}$. Given an $\mathbb{A}^{i-1}$-diagram $\mathcal{H}^{i-1}$ for $\mathcal{T}^{i-1}$, we construct an $\mathbb{A}^{i}$-diagram for $\mathcal{T}^{i}$ as follows. As before, suppose $J_{i}$ connectes $T_{a}^{i-1}$ to $T_{b}^{i-1}$.

(1) The Heegaard surface $\Sigma^{i}$ is obtained from $\Sigma^{i-1}$ by adding a one-handle with feet near $z_{a}$ and $z_{b}$, where $z_{a}=T_{\bullet}^{i-1} \cap \Sigma^{i-1}$ for $\bullet=a, b$.

(2) $\boldsymbol{\alpha}^{i}=\boldsymbol{\alpha}^{i-1} \cup\left\{\alpha_{i}\right\}$ where $\alpha_{i}$ is the core of the attached one-handle.

(3) $\boldsymbol{\beta}^{i}=\boldsymbol{\beta}^{i-1} \cup\left\{\beta_{i}\right\}$ where $\beta_{i}$ is a Hamiltonian translate of $\alpha_{i}$, intersecting $\alpha_{i}$ in a pair of canceling intersection points. The area bounded between $\alpha_{i}$ and $\beta_{i}$ gives a 2-chain $\mathcal{P}=D_{i}^{+}-D_{i}^{-}$with $\partial \mathcal{P}=\alpha_{i}-\beta_{i}$, the components $D_{i}^{+}$and $D_{i}^{-}$of $\Sigma^{i}-\boldsymbol{\alpha}^{i}-\boldsymbol{\beta}^{i}$ are bigons such that one of them intersects $\mathbf{z}$ in $z_{a}$ while the other one intersects $\mathbf{z}$ in $z_{b}$.

(4) $\mathbf{z}^{i}=\mathbf{z}^{i-1}$ and $\overline{\mathbf{z}}^{i}=\overline{\mathbf{z}}^{i-1}$.

As a result, we get an $\mathbb{A}_{F}^{b}$-diagram

$$
\mathcal{H}:=\mathcal{H}^{n}=\left(\Sigma, \boldsymbol{\alpha}=\left\{\alpha_{1}, \ldots, \alpha_{n}\right\}, \boldsymbol{\beta}=\left\{\beta_{1}, \ldots, \beta_{n}\right\}, \mathfrak{u}_{F}^{b}: \mathbf{z} \amalg \overline{\mathbf{z}} \rightarrow \mathbb{A}_{F}^{b}, \mathfrak{s}_{0}\right)
$$

for $\mathcal{T}^{b}$. Any pair of curves $\left(\alpha_{i}, \beta_{i}\right)$ intersect in a pair of points $x_{i}^{+}$and $x_{i}^{-}$, so that the bi-gons $D_{i}^{+}$ and $D_{i}^{-}$connect $x_{i}^{+}$to $x_{i}^{-}$. Thus, corresponding to any map $\epsilon:\{1, \ldots, n\} \rightarrow\{+,-\}$ we have an intersection point

$$
\mathbf{x}^{\epsilon}=\left\{x_{1}^{\epsilon(1)}, \ldots, x_{n}^{\epsilon(n)}\right\} \in \mathbb{T}_{\alpha} \cap \mathbb{T}_{\beta} .
$$

By an argument similar to the argument of Proposition 5.2, the map

$$
f_{i}: \mathrm{CF}\left(\Sigma^{i-1}, \boldsymbol{\alpha}^{i-1}, \boldsymbol{\beta}^{i-1}, \mathfrak{u}^{i-1}, \mathfrak{s}_{0}^{i-1}\right) \otimes \mathbb{A}^{i} \rightarrow \mathrm{CF}\left(\Sigma^{i}, \boldsymbol{\alpha}^{i}, \boldsymbol{\beta}^{i}, \mathfrak{u}^{i}, \mathfrak{s}_{0}^{i}\right)
$$

which is defined by $f_{i}(\mathbf{y})=\mathbf{y} \times\left\{x_{i}^{+}\right\}$, is a chain map if the path of almost complex structures is chosen correctly. In particular, the intersection point $\theta^{+}:=\left\{x_{1}^{+}, \ldots, x_{n}^{+}\right\}$is closed in the chain complex

i.e. we have $\partial \theta^{+}=0$.

$$
\mathrm{CF}\left(\Sigma, \boldsymbol{\alpha}, \boldsymbol{\beta}, \mathfrak{u}_{F}^{b}, \mathfrak{s}_{0}\right)=\mathrm{CF}\left(\mathcal{T}^{b}\right)
$$

For every $1 \leq i \leq n$, let $\left(F_{i}^{\prime}, \partial F_{i}^{\prime}\right) \subset\left(M^{i}, \partial M^{i} \cup T^{i} \cup \bar{T}^{i}\right)$ denote the embedded, oriented surface obtained from $D$ by attaching the embedded one handles $D_{1}^{\prime}, \ldots, D_{i}^{\prime}$. Each $F_{i}^{\prime}$ is then a decomposing surface in $\left(M^{i}, T^{i} \amalg \bar{T}^{i}\right)$. We may inductively construct a closed subsurface $P^{i} \subset \Sigma^{i}$ such that the Heegaard diagram $\mathcal{H}^{i}$, together with $P^{i}$, is a diagram adapted to $F_{i}^{\prime}$ in the sense of Juh08, Definition 4.3]. More precisely, $P^{i}$ is a closed subsurface of $\Sigma^{i}$ such that the boundary of $P^{i}$ is a union of polygons, whose vertices are $P^{i} \cap(\mathbf{z} \amalg \overline{\mathbf{z}})$ and its edges are decomposed as $\partial P^{i}=A^{i} \cup B^{i}$ where

$$
A^{i} \cap B^{i} \subset \mathbf{z} \amalg \overline{\mathbf{z}}, \quad \boldsymbol{\alpha}^{i} \cap B^{i}=\emptyset \quad \text { and } \quad \boldsymbol{\beta}^{i} \cap A^{i}=\emptyset .
$$

Finally, the equivalence class of $F_{i}^{\prime}$ is given by smoothing the corners of

$$
\left(P^{i} \times\{1 / 2\}\right) \cup\left(A^{i} \times[1 / 2,1]\right) \cup\left(B^{i} \times[0,1 / 2]\right) \subset\left(M^{i}, T^{i} \amalg \bar{T}^{i}\right) .
$$

For every $i$, suppose that $J_{i}$ connects $T_{a_{i}}^{i-1}$ to $T_{b_{i}}^{i-1}$. For $i=1, F_{1}^{\prime}$ is obtained from $D$ by attaching a one-handle to $T_{a_{1}}^{0}$ and $T_{b_{1}}^{0}$. Thus, $P^{1}$ is a union of a rectangle whose vertices are $z_{a_{1}}, z_{b_{1}}, \bar{z}_{a_{1}}$ and $\bar{z}_{b_{1}}$ and contains the intersection point $x_{1}^{-}$, together with $n-2$ bigons disjoint from $\boldsymbol{\alpha}^{1}$ and $\boldsymbol{\beta}^{1}$, whose vertices are $z_{j}$ and $\bar{z}_{j}$ for $j \neq a_{1}, b_{1}$. For any $i>1$, the subsurface $P^{i}$ may be constructed from $P^{i-1}$ by attaching an embedded one-handle in $\Sigma^{i}$ which intersects $\alpha_{i}$ and $\beta_{i}$ and contains $x_{i}^{-}$, as illustrated in Figure 5 . 


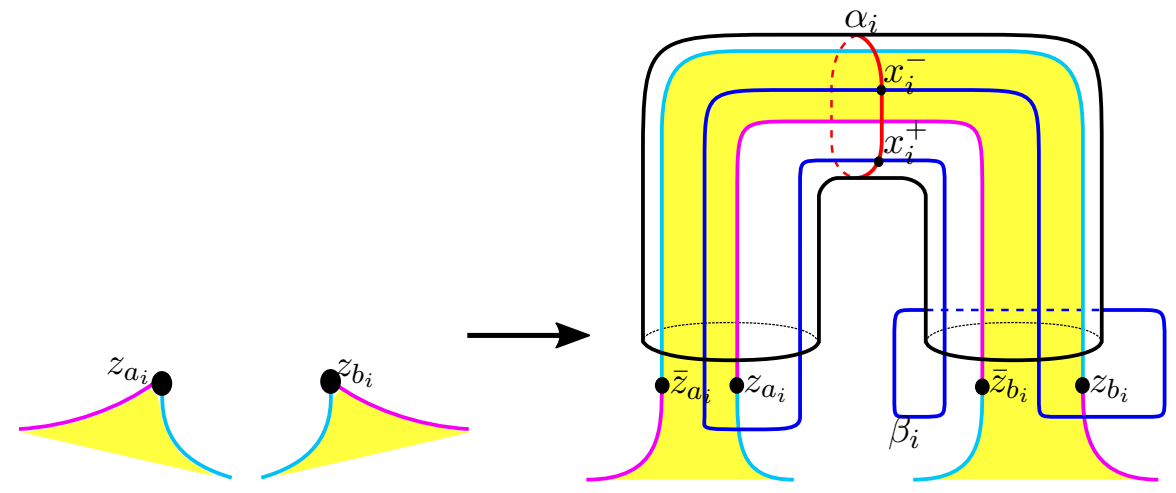

FiguRE 5 . The $i$-th one-handle is attached over the marked points $z_{a_{i}}$ and $z_{b_{i}}$. The curves $\alpha_{i}$ and $\beta_{i}$, their intersection points $x_{i}^{+}$and $x_{i}^{-}$and the modification changing $P^{i-1}$ to $P^{i}$ are illustrated.

Thus, for $i=n, \theta^{+}$is the only intersection point that does not intersect $P^{n}$. As a result, $\theta^{+}$is the only intersection point for which $\underline{\mathfrak{s}}\left(\theta^{+}\right)=\underline{\mathfrak{s}}_{F^{\prime}}$ and thus $\theta^{+}$is the generator of

$$
\operatorname{SFH}\left(X_{F}, \tau_{F}^{b}, \underline{\mathfrak{s}}_{F^{\prime}}\right)=\mathbb{F} \text {. }
$$

For every $\epsilon:\{1, \ldots, n\} \rightarrow\{+,-\}$, there is a positive disk

$$
\phi \in \pi_{2}\left(\theta^{+}, \mathbf{x}^{\epsilon}\right) \text { with } \mu(\phi)=\#\{i \mid \epsilon(i)=-\} .
$$

Furthermore, $\underline{\mathfrak{s}}\left(\mathfrak{u}_{F}^{b}(\phi) \cdot \mathbf{x}^{\epsilon}\right)=\underline{\mathfrak{s}}_{F^{\prime}}$ and if for a monomial $\mathfrak{u} \in \mathbb{A}_{F}^{b}$ we have $\underline{\mathfrak{s}}\left(\mathfrak{u} \cdot \mathbf{x}^{\epsilon}\right)=\underline{\mathfrak{s}}_{F^{\prime}}$ then $\mathfrak{u}=$ $\mathfrak{u}_{F}^{b}(\phi) . \widetilde{\mathfrak{u}}$ for some $\widetilde{\mathfrak{u}} \in \mathbb{A}_{F}^{b}$. Hence $\Theta_{F}^{b}=\theta^{+}$, which is closed by our earlier considerations, generates the top-dimensional homology group in

$$
\operatorname{HF}\left(M_{F}, T_{F}^{b}, \mathfrak{u}_{F}^{b}, \underline{\mathfrak{s}}_{F^{\prime}}\right)
$$

with respect to the relative grading defined above. The above observations complete the proof of the proposition.

The algebra $\mathbb{A}$ has a natural $\mathbb{A}_{F}^{b}$-module structure given by the homomorphism $\phi: \mathbb{A}_{F}^{b} \rightarrow \mathbb{A}$ defined as

$$
\begin{cases}\phi\left(\mathfrak{u}_{i}\right)=\mathfrak{u}_{F}\left(F_{i}\right) & 1 \leq i \leq m \\ \phi\left(\mathfrak{v}_{i}\right)=1 & 1 \leq i \leq \kappa .\end{cases}
$$

Further, considering this module structure on $\mathbb{A}$ it is straightforward that

$$
\operatorname{HF}^{\mathbb{A}}\left(\mathcal{T}^{b}\right)=\operatorname{HF}\left(\mathcal{T}_{F}\right)
$$

Definition 6.3. The image of the homolog class $\Theta_{F}^{b} \otimes 1$ under the homomorphism

$$
\operatorname{HF}\left(\mathcal{T}^{b}\right) \otimes_{\phi} \mathbb{A} \rightarrow \mathrm{H}_{\star}\left(\mathrm{CF}\left(\mathcal{T}^{b}\right) \otimes_{\phi} \mathbb{A}\right)=\operatorname{HF}^{\mathbb{A}}\left(\mathcal{T}^{b}\right)=\operatorname{HF}\left(\mathcal{T}_{F}\right)
$$

is denoted by $\Theta_{F}$ and is called the distinguished generator of $\operatorname{HF}\left(\mathcal{T}_{F}\right)$.

6.3. Framed arcs, framed knots and the cobordism map. Let $(M, T)$ be a balanced tangle, $\mathbb{I}=\left\{\mathbb{I}_{1}, \ldots, \mathbb{I}_{n}\right\}$ be an acceptable set of framed arcs in $(M, T)$ and $\mathbb{S}=\left\{\mathbb{S}_{1}, \ldots, \mathbb{S}_{m}\right\}$ be a set of framed circles in $M \backslash(\mathbb{I} \cup T)$. In other words, each $\mathbb{S}_{i}$ is determined by a framing on a knot $K_{i}$ and each $\mathbb{I}_{i}$ is determined by a framing on an arc $I_{i}$. Let $L=\amalg_{i=1}^{m} K_{i}$ and $I=\amalg_{i=1}^{n} I_{i}$. As discussed in Section 4.1. $(\mathbb{I}, \mathbb{S})$ specifies a cobordism $\mathcal{W}=(W, F)=\mathcal{W}(\mathbb{I}, \mathbb{S})$ from $(M, T)$ to $\left(M^{\prime}, T^{\prime}\right)=(M(\mathbb{S}), T(\mathbb{I}))$. Here, $T(\mathbb{I})$ is constructed from band surgery on $T$ along $\mathbb{I}$ and $M(\mathbb{S})$ is constructed from $M$ by surgery along the framed link $\mathbb{S}$. 
Definition 6.4. With the above notation fixed, a stalk $s(\mathbb{S})=\left\{s_{1}, \ldots, s_{m}\right\}$ for the framed link $\mathbb{S}$ (disjoint from $\mathbb{I})$ in $(M, T)$ is a set of embedded arcs in $M \backslash(T \cup \mathbb{I})$ such that $s_{i}$ connects $K_{i}$ to $\partial^{+} M \backslash T$.

For every $i$, if $\mathbb{I}$ intersects $T_{i}$, let $r_{i} \subset T_{i}$ be the segment where

$$
\partial^{+} r_{i} \subset \partial^{+} M, \quad \partial^{-} r_{i} \subset T_{i} \cap \mathbb{I} \quad \text { and } \quad\left(T_{i}-r_{i}\right) \cap \mathbb{I}=\emptyset .
$$

If $T_{i} \cap \mathbb{I}=\emptyset$, let $r_{i}=\emptyset$ and denote $r(\mathbb{I})=\amalg_{i=1}^{\kappa} r_{i}$. Let

$$
B(\mathbb{I})=I \cup r(\mathbb{I}) \quad \text { and } \quad B(\mathbb{S})=L \cup s(\mathbb{S}) .
$$

Consider small tubular neighborhoods $\operatorname{nd}(r(\mathbb{I}))$ and $\operatorname{nd}(s(\mathbb{S}))$ of $r(\mathbb{I})$ and $s(\mathbb{S})$, respectively and let

$$
N=\mathbb{I} \cup \mathbb{S} \cup \operatorname{nd}(r(\mathbb{I})) \cup \operatorname{nd}(s(\mathbb{S}))
$$

be the resulting neighborhood of $B=B(\mathbb{I}) \cup B(\mathbb{S})$. For each $i=1, \ldots, n$, let $A_{i} \subset \partial N$ be a sphere with 4 boundary components resulted from the intersection of an enlarged neighborhood of $I_{i}$ with $\partial N$. Such neighborhoods are illustrated in Figure 6. The intersection of $T$ with $M^{\prime \prime}=M-N$ defines a new tangle $\left(M^{\prime \prime}, T^{\prime \prime}=T \cap M^{\prime \prime}\right)$.

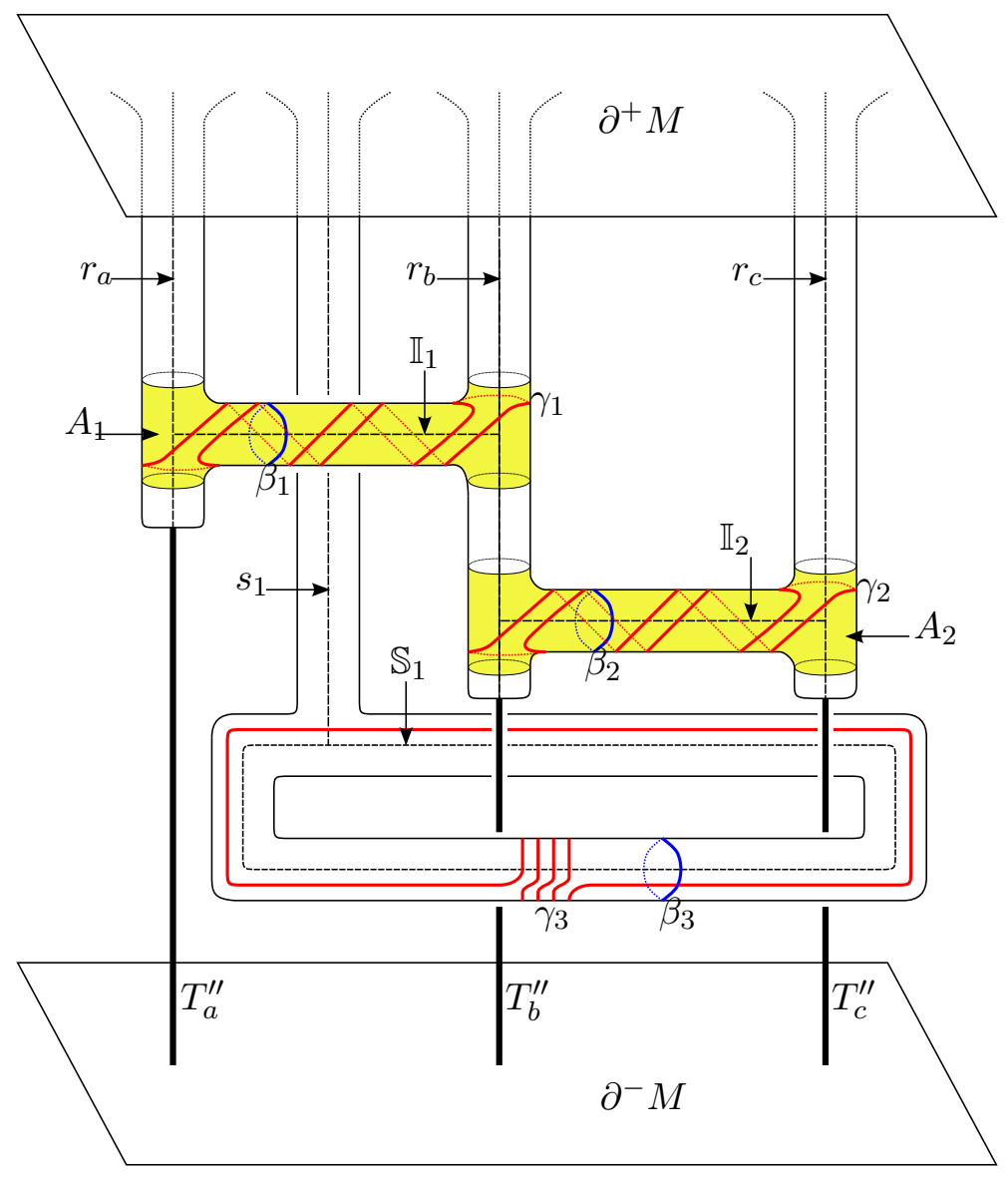

Figure 6. A Heegaard diagram subordinate to a framed knot $\mathbb{S}_{1}$ and a pair of framed arcs $\mathbb{I}_{1}$ and $\mathbb{I}_{2}$ with one end point on the same strand $T_{b}$ of $T$, and the other ends on the strands $T_{a}$ and $T_{c}$. A tubular neighborhood of the union of $K_{1}, s_{1}, I_{1}, I_{2}, r_{a}, r_{b}$ and $r_{c}$ is deleted to obtain the tangle $\left(M^{\prime \prime}, T^{\prime \prime}\right)$. Attaching disks to the meridians $\beta_{1}$ and $\beta_{2}$ of $\mathbb{I}_{1}$ and $\mathbb{I}_{2}$ and the meridian $\beta_{3}$ of $\mathbb{S}_{1}$ gives a Heegaard diagram for $(M, T)$ while the framings of $\mathbb{I}_{1}, \mathbb{I}_{2}$ and $\mathbb{S}_{1}$ determine the curves $\gamma_{1}, \gamma_{2}$ and $\gamma_{3}$. The curves $\gamma_{1}$ and $\gamma_{2}$ live in the 4-punctured spheres $A_{1}$ and $A_{2}$, respectively. 
Definition 6.5. A Heegaard triple subordinate to the framed arcs $\mathbb{I}$, the framed link $\mathbb{S}$, and the stalk $s(\mathbb{S})$ for the balanced tangle $(M, T)$ is a Heegaard triple

$$
\left(\Sigma, \boldsymbol{\alpha}=\left\{\alpha_{1}, \ldots, \alpha_{\ell}\right\}, \boldsymbol{\beta}=\left\{\beta_{1}, \ldots, \beta_{\ell}\right\}, \gamma=\left\{\gamma_{1}, \ldots, \gamma_{\ell}\right\}, \mathbf{z}\right)
$$

satisfying the following conditions:

(1) $\left(\Sigma, \boldsymbol{\alpha},\left\{\beta_{n+m+1}, \ldots, \beta_{\ell}\right\}, \mathbf{z}\right)$ is a Heegaard diagram for $\left(M^{\prime \prime}, T^{\prime \prime}\right)$. Fix an identification of $\Sigma\left[\beta_{n+m+1}, \ldots, \beta_{\ell}\right]$ with $\partial^{+} M^{\prime \prime}$.

(2) For $i=m+n+1, \ldots, \ell, \gamma_{i}$ is obtained by a small Hamiltonian isotopy from $\beta_{i}$ supported away from the marked points, so that $\left|\beta_{i} \cap \gamma_{i}\right|=2$.

(3) For any $i=1, \ldots, m$, the curves $\beta_{n+i}$ and $\gamma_{n+i}$ lie on the punctured torus $\partial \mathbb{S}_{i} \cap \partial^{+} M^{\prime \prime}$, representing the meridian and the framing of $K_{i}$, respectively. Further, they meet in a single transverse intersection point.

(4) For any $j=1, \ldots, n$, the curves $\beta_{j}$ and $\gamma_{j}$ lie on the punctured sphere $A_{j}$. Moreover, $\beta_{j}$ represents the meridian of $\mathbb{I}_{j}$ and meets $\gamma_{j}$ in two transverse intersection points, while $\gamma_{j}$ is obtained from $\beta_{j}$ by an isotopy corresponding to the framing of $\mathbb{I}_{j}$ (which crosses two of the boundary components of $A_{j}$ ), as illustrated in Figure 6 .

(5) The Heegaard diagrams $(\Sigma, \boldsymbol{\alpha}, \boldsymbol{\beta}, \mathbf{z})$ and $(\Sigma, \boldsymbol{\alpha}, \boldsymbol{\gamma}, \mathbf{z})$ are diagrams for $(M, T)$ and $\left(M^{\prime}, T^{\prime}\right)$, respectively.

We say that a Heegaard triple is subordinate to the framed arcs $\mathbb{I}$ and the framed link $\mathbb{S}$ if it is subordinate to the framed arcs $\mathbb{I}$ and the framed link $\mathbb{S}$ and some stack $s(\mathbb{S})$ for $\mathbb{S}$.

The existence of Heegaard triples subordinate to an acceptable set of framed arcs $\mathbb{I}$ and a framed link $\mathbb{S}$ for a stable cobordism $\mathcal{W}$ as above and the correspondence between different such Heegaard triples is addressed in the following lemma.

Lemma 6.6. Let $\mathbb{I}=\left\{\mathbb{I}_{1}, \ldots, \mathbb{I}_{n}\right\}$ be an acceptable set of framed arcs in $(M, T)$ and $\mathbb{S}=\left\{\mathbb{S}_{1}, \ldots, \mathbb{S}_{m}\right\}$ be a framed link in $M \backslash(T \cup \mathbb{I})$. There is a Heegaard triple subordinate to $\mathbb{I}$ and $\mathbb{S}$. Further, every two such triples may be connected (after composing with a diffeomorphism of the diagram) by a sequence of following moves, all supported away from the set $\mathbf{z}$ of marked points:

(1) Isotopies and handle slides among $\left\{\alpha_{1}, \ldots, \alpha_{\ell}\right\}$,

(2) Isotopies and handle slides among $\left\{\beta_{n+m+1}, \ldots, \beta_{\ell}\right\}$ while carrying the corresponding isotopy or handle slide among $\left\{\gamma_{n+m+1}, \ldots, \gamma_{\ell}\right\}$,

(3) Stabilization (and destabilization); i.e. taking the connected sum of the Heegaard triple with a triple $(E, \alpha, \beta, \gamma)$, where $E$ is a surface of genus one, $|\alpha \cap \beta|=1$, and $\gamma$ is obtained by a small Hamiltonian isotopy from $\beta$ such that $|\beta \cap \gamma|=2$,

(4) Isotopies or handle slides of $\beta_{n+j}$ along the curves in $\left\{\beta_{n+m+1}, \ldots, \beta_{\ell}\right\}$ for $j=1, \ldots, m$,

(5) Isotopy or handle slides of $\gamma_{n+j}$ along the curves in $\left\{\gamma_{n+m+1}, \ldots, \gamma_{\ell}\right\}$ for $j=1, \ldots, m$,

(6) For $i=1, \ldots, n$, isotopy or handle slide of $\beta_{i}$ along the curves $\left\{\beta_{n+m+1}, \ldots, \beta_{\ell}\right\}$, while carrying the corresponding isotopy or handle slide on $\gamma_{i}$,

(7) Handle slide of a curve in $\left\{\beta_{n+m+1}, \ldots, \beta_{\ell}\right\}$ along some $\beta_{n+j}$ for $j=1, \ldots, m$, while doing a handle slide of the corresponding curve in $\left\{\gamma_{n+m+1}, \ldots, \gamma_{\ell}\right\}$ along $\gamma_{n+j}$.

Proof. Given the stack $s(\mathbb{S})$ for $\mathbb{S}$, the proof of Lemma 4.5 from [OS06 may be used to show that Heegaard diagrams subordinate to $\mathbb{I}, \mathbb{S}$ and $s(\mathbb{S})$ exist and that for every pair $H$ and $H^{\prime}$ of such diagrams, $H$ may be changed to $H^{\prime}$ via a sequence of moves of types $1,2,3,4,5$ and 6 in the statement of the lemma. If the stacks $s(\mathbb{S})$ and $s^{\prime}(\mathbb{S})$ are different, the proof of [OS06, Lemma 4.8] implies that there is a Heegaard diagram $H$ as above subordinate to $\mathbb{I}, \mathbb{S}$ and $s(\mathbb{S})$ and a Heegaard diagram $H^{\prime}$ subordinate to $\mathbb{I}, \mathbb{S}$ and the stalk $s^{\prime}(\mathbb{S})$ such that the following is true. There is a sequence of handle slides of some particular curves in $\left\{\beta_{n+m+1}, \ldots, \beta_{\ell}\right\}$ over the curves $\beta_{n+j}$ for $j=1, \ldots, m$ and other curves in $\left\{\beta_{n+m+1}, \ldots, \beta_{\ell}\right\}$ (and a corresponding sequence of handle slides for $\gamma$ ) which change $H$ to $H^{\prime}$. The lemma then follows. 
Let $H=(\Sigma, \boldsymbol{\alpha}, \boldsymbol{\beta}, \boldsymbol{\gamma}, \mathbf{z})$ be a Heegaard triple subordinated to the framed arcs $\mathbb{I}$ and the framed link $\mathbb{S}$ as above. Associated with $H$ we have a cobordism

$$
\mathcal{W}_{H}=\left(W_{H}, F_{H}\right):(M, T) \amalg\left(M_{\beta \gamma}, T_{\beta \gamma}\right) \leadsto(M(\mathbb{S}), T(\mathbb{I})),
$$

where $\left(M_{\beta \gamma}, T_{\beta \gamma}\right)$ is the balanced tangle determined by the Heegaard diagram $(\Sigma, \boldsymbol{\beta}, \boldsymbol{\gamma}, \mathbf{z})$. This cobordism is related to $\mathcal{W}(\mathbb{I}, \mathbb{S})=(W, F)$ as follows.

Each $\mathbb{I}_{i}$ determines an embedded arc $J_{i}$ on $F$ with endpoints on $T$. After applying a smooth isotopy supported in a neighborhood of $\partial F$ on $J=\left\{J_{1}, \ldots, J_{n}\right\}$ which moves the endpoints to $\partial^{+} F$, it becomes a spanning set of $\operatorname{arcs}$ on $F$. Let $\operatorname{nd}(J)$ be a tubular neighborhood of $J$. Then,

$$
(W-\operatorname{nd}(J), F \cap(W-\operatorname{nd}(J)))
$$

gives a cobordism from $(M, T) \amalg\left(M_{F}, T_{F}\right)$ to $(M(\mathbb{S}), T(\mathbb{I}))$.

Lemma 6.7. Under the above assumptions, after attaching 3 -handles along $S_{1}, \ldots, S_{\ell-n-m} \subset M_{\beta \gamma}$ to $\mathcal{W}_{H}$, we obtain the cobordism

$$
(W-\operatorname{nd}(J), F \cap(W-\operatorname{nd}(J))) .
$$

Proof. Denote the compression body obtained by attaching disks to $\Sigma \times[0,1]$ along $\boldsymbol{\beta} \times\{1\}$ by $C(\boldsymbol{\beta})$. Without loss of generality, we may assume that

$$
\mathbb{I}_{i}, \mathbb{S}_{j} \subset C(\boldsymbol{\beta}) \quad \text { for } i \in\{1, \ldots, n\}, j \in\{1, \ldots, m\} .
$$

Furthermore, we may consider an identification

$$
W=(M \times[0,1]) \cup_{\{1\} \times \mathbb{S}}\left(\bigcup_{i=1}^{m} D^{2} \times D^{2}\right)
$$

such that $\left.\pi_{2}\right|_{F}$ is a Morse function with all critical points of index one. Here, $\pi_{2}$ is the projection map from $M \times[0,1] \backslash\{1\} \times \mathbb{S}$ onto [0,1]. Moreover, we assume that

$$
\operatorname{Crit}\left(\left.\pi_{2}\right|_{F}\right) \subset M \times\{1 / 2\} \text {. }
$$

Thus, $T_{1 / 2}=F \cap(M \times\{1 / 2\})$ is a properly embedded, oriented, singular 1-dimensional submanifold of $M \times\{1 / 2\}$. For every framed arc $\mathbb{I}_{i}$ we have a singular point

$$
q_{i} \in T_{1 / 2} \cap(C(\boldsymbol{\beta}) \times\{1 / 2\}) \subset F .
$$

Let $B^{\prime}(\mathbb{I}) \subset T_{1 / 2}$ be the subspace corresponding to $B(\mathbb{I})$. Let $N^{\prime}, N_{1 / 2} \subset W$ be tubular neighborhoods of $B^{\prime}(\mathbb{I})$ and $C(\boldsymbol{\beta}) \times\{1 / 2\}$, respectively. Then

$$
\begin{aligned}
& \left(W-N^{\prime}, F \cap\left(W-N^{\prime}\right)\right)=(W-\operatorname{nd}(J), F \cap(W-\operatorname{nd}(J))) \text { and } \\
& \left(W-N_{1 / 2}, F \cap\left(W-N_{1 / 2}\right)\right)=\mathcal{W}_{H} .
\end{aligned}
$$

Thus, $(W-\operatorname{nd}(J), F \cap(W-\operatorname{nd}(J)))$ is obtained from $\mathcal{W}_{H}$ by attaching 3-handles along the spheres $S_{1}, \ldots, S_{\ell-n-m} \subset M_{\beta \gamma}$.

It follows from the proof of Lemma 6.7 that $\left(M_{F}, T_{F}\right)$ is obtained by surgery on $\left(M_{\beta \gamma}, T_{\beta \gamma}\right)$ along the 2 -spheres $S_{1}, \ldots, S_{\ell-m-n}$. Abusing the notation, let $\mathfrak{s}_{0} \in \operatorname{Spin}^{c}\left(M_{\beta \gamma}\right)$ denote the $\operatorname{Spin}^{c}$ class obtained from $\mathfrak{s}_{0} \in \operatorname{Spin}^{c}\left(M_{F}\right)$ which satisfies

$$
\left\langle c_{1}\left(\mathfrak{s}_{0}\right), S_{i}\right\rangle=0 \quad \text { for any } i \in\{1,2, \ldots, \ell-n-m\} .
$$

For any Heegaard tripe $H$ subordinated to $\mathbb{I}$ and $\mathbb{S}$, let

$$
r: \operatorname{Spin}^{c}(\mathcal{W}(\mathbb{I}, \mathbb{S})) \rightarrow \operatorname{Spin}^{c}\left(\mathcal{W}_{H}\right)
$$

denote the restriction map.

Lemma 6.8. For every $\mathfrak{t} \in \operatorname{Spin}^{c}(\mathcal{W}(\mathbb{I}, \mathbb{S}))$, the restriction $\left.r(\mathfrak{t})\right|_{M_{\beta \gamma}}$ is the $\operatorname{Spin}^{c}$ class $\mathfrak{s}_{0} \in \operatorname{Spin}^{c}\left(M_{\beta \gamma}\right)$.

Proof. This is straightforward. 
Suppose $\mathfrak{u}_{F}: \pi_{0}(F) \rightarrow \mathbb{A}$ be an $\mathbb{A}$-coloring for $\mathcal{W}(\mathbb{I}, \mathbb{S})$. Given a $\operatorname{Spin}^{c}$ class $\mathfrak{t} \in \operatorname{Spin}^{c}(\mathcal{W}(\mathbb{I}, \mathbb{S}))$ we abuse the notation and call the Heegaard triple $(\Sigma, \boldsymbol{\alpha}, \boldsymbol{\beta}, \boldsymbol{\gamma}, \mathfrak{u}) \mathfrak{t}$-admissible, if it is $r(\mathfrak{t})$-admissible. Here $\mathfrak{u}: \mathbf{z} \rightarrow \mathbb{A}$ denotes the map induced by $\mathfrak{u}_{F}$. Note that every diagram $(\Sigma, \boldsymbol{\alpha}, \boldsymbol{\beta}, \boldsymbol{\gamma}, \mathfrak{u})$ subordinate to $\mathbb{I}$ and $\mathbb{S}$ may be transformed to a $\mathfrak{t}$-admissible Heegaard diagram by performing isotopies on the curves in $\boldsymbol{\alpha}$, following a procedure similar to [AE15, Section 4.2]. Let us assume that the Heegaard diagram $(\Sigma, \boldsymbol{\alpha}, \boldsymbol{\beta}, \boldsymbol{\gamma}, \mathfrak{u})$ subordinate to $\mathbb{I}$ and $\mathbb{S}$ is $\mathfrak{t}$-admissible. We then call $\mathcal{H}=(\Sigma, \boldsymbol{\alpha}, \boldsymbol{\beta}, \boldsymbol{\gamma}, \mathfrak{u}, \mathfrak{t})$ an $\mathbb{A}$-diagram for

$$
\mathcal{C}=\mathcal{C}(\mathbb{I}, \mathbb{S})=\left[W, F, \mathfrak{t}, \mathfrak{u}_{F}\right]: \mathcal{T}=\left[M, T, \mathfrak{s}=\left.\mathfrak{t}\right|_{M}, \mathfrak{u}_{T}\right] \leadsto \mathcal{T}^{\prime}=\left[M^{\prime}, T^{\prime}, \mathfrak{s}^{\prime}=\left.\mathfrak{t}\right|_{M^{\prime}}, \mathfrak{u}_{T}^{\prime}\right],
$$

subordinate to $\mathbb{I}$ and $\mathbb{S}$. Here, $\mathfrak{u}_{T}$ and $\mathfrak{u}_{T}^{\prime}$ are the $\mathbb{A}$-colorings induced by $\mathfrak{u}_{F}$ on $(M, T)$ and $\left(M^{\prime}, T^{\prime}\right)$, respectively.

For an appropriate generic family of almost complex structures $J$, the $\mathbb{A}$-diagram $\mathcal{H}$ determines a holomorphic triangle map

$$
\mathfrak{f}_{\alpha \beta \gamma}: \operatorname{HF}_{J}(\Sigma, \boldsymbol{\alpha}, \boldsymbol{\beta}, \mathfrak{u}, \mathfrak{s}) \otimes_{\mathbb{A}} \operatorname{HF}_{J}\left(\Sigma, \boldsymbol{\beta}, \boldsymbol{\gamma}, \mathfrak{u}, \mathfrak{s}_{0}\right) \longrightarrow \operatorname{HF}_{J}\left(\Sigma, \boldsymbol{\alpha}, \boldsymbol{\gamma}, \mathfrak{u}, \mathfrak{s}^{\prime}\right)
$$

From the Heegaard diagram $(\Sigma, \boldsymbol{\beta}, \boldsymbol{\gamma}, \mathbf{z})$ for $\left(M_{\beta \gamma}, T_{\beta \gamma}\right)$ we may construct a Heegaard diagram $H^{\prime}=\left(\Sigma^{\prime}, \boldsymbol{\beta}^{\prime}, \boldsymbol{\gamma}^{\prime}, \mathbf{z}\right)$ for $\left(M_{F}, T_{F}\right)$ where $\Sigma^{\prime}$ is obtained by surgery on $\Sigma$ along $\beta_{n+m+1}, \ldots, \beta_{\ell}$, while

$$
\boldsymbol{\beta}^{\prime}=\left\{\beta_{1}, \ldots, \beta_{n+m}\right\} \text { and } \boldsymbol{\gamma}^{\prime}=\left\{\gamma_{1}, \ldots, \gamma_{n+m}\right\} .
$$

Following the construction in Section 5.3 , we get a homomorphism

$$
\mathfrak{f}_{\beta \gamma}: \operatorname{HF}\left(\Sigma^{\prime}, \boldsymbol{\beta}^{\prime}, \boldsymbol{\gamma}^{\prime}, \mathfrak{u}, \mathfrak{s}_{0}\right) \rightarrow \operatorname{HF}\left(\Sigma, \boldsymbol{\beta}, \boldsymbol{\gamma}, \mathfrak{u}, \mathfrak{s}_{0}\right) .
$$

Then, we set $\Theta_{\beta \gamma}:=\mathfrak{f}_{\beta \gamma}\left(\Theta_{F}\right)$ and define

$$
\begin{aligned}
& \mathfrak{f}_{\mathcal{H}, J}: \operatorname{HF}_{J}(\Sigma, \boldsymbol{\alpha}, \boldsymbol{\beta}, \mathfrak{u}, \mathfrak{s}) \longrightarrow \operatorname{HF}_{J}\left(\Sigma, \boldsymbol{\alpha}, \boldsymbol{\gamma}, \mathfrak{u}, \mathfrak{s}^{\prime}\right) \\
& \mathfrak{f}_{\mathcal{H}, J}(\mathbf{x}):=\mathfrak{f}_{\alpha \beta \gamma}\left(\mathbf{x} \otimes \Theta_{\beta \gamma}\right) \quad \text { for any } \quad \mathbf{x} \in \operatorname{HF}_{J}(\Sigma, \boldsymbol{\alpha}, \boldsymbol{\beta}, \mathfrak{u}, \mathfrak{s}) .
\end{aligned}
$$

Theorem 6.9. For any $\mathbb{A}$-module $\mathbb{M}$, the homomorphism $\mathfrak{f}_{\mathcal{H}, J}$ induces a homomorphism

$$
\mathfrak{f}_{\mathcal{C}, \mathbb{I}, \mathbb{S}}^{\mathbb{M}}: \operatorname{HF}^{\mathbb{M}}(\mathcal{T}) \rightarrow \operatorname{HF}^{\mathbb{M}}\left(\mathcal{T}^{\prime}\right) .
$$

6.4. Proof of Theorem 6.9. It follows from standard arguments in Floer theory that for appropriate families of almost complex structures $J$ and $J^{\prime}$, we have

$$
\mathfrak{f}_{\mathcal{H}, J^{\prime}} \circ \Phi_{J \rightarrow J^{\prime}}=\Phi_{J \rightarrow J^{\prime}} \circ \mathfrak{f}_{\mathcal{H}, J} .
$$

So we denote the induced map by $\mathfrak{f}_{\mathcal{H}}$. Let

$$
\mathcal{H}=(\Sigma, \boldsymbol{\alpha}, \boldsymbol{\beta}, \boldsymbol{\gamma}, \mathfrak{u}, \mathfrak{t}) \text { and } \mathcal{H}^{\prime}=\left(\Sigma^{\prime}, \boldsymbol{\alpha}^{\prime}, \boldsymbol{\beta}^{\prime}, \boldsymbol{\gamma}^{\prime}, \mathfrak{u}^{\prime}, \mathfrak{t}\right)
$$

be $\mathbb{A}$-diagrams for $\mathcal{C}$ subordinate to $\mathbb{I}$ and $\mathbb{S}$. Assume that $\mathcal{H}^{\prime}$ is obtained from $\mathcal{H}$ by one Heegaard move $e$ of the type specified in Lemma 6.6. Associated with $e$, let

$$
\begin{aligned}
& \Phi_{e}: \operatorname{HF}^{\mathbb{M}}(\Sigma, \boldsymbol{\alpha}, \boldsymbol{\beta}, \mathfrak{u}, \mathfrak{s}) \rightarrow \operatorname{HF}^{\mathbb{M}}\left(\Sigma^{\prime}, \boldsymbol{\alpha}^{\prime}, \boldsymbol{\beta}^{\prime}, \mathfrak{u}^{\prime}, \mathfrak{s}\right), \\
& \Psi_{e}: \operatorname{HF}^{\mathbb{M}}\left(\Sigma, \boldsymbol{\alpha}, \boldsymbol{\gamma}, \mathfrak{u}, \mathfrak{s}^{\prime}\right) \rightarrow \operatorname{HF}^{\mathbb{M}}\left(\Sigma^{\prime}, \boldsymbol{\alpha}^{\prime}, \boldsymbol{\gamma}^{\prime}, \mathfrak{u}^{\prime}, \mathfrak{s}^{\prime}\right) .
\end{aligned}
$$

be the isomorphisms defined in Section 3.3 . We need to prove that the diagram

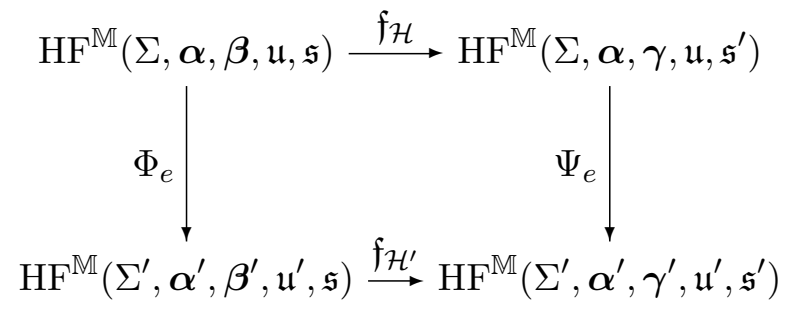

commutes. Let us first consider the Heegaard move $e$ which changes $\boldsymbol{\alpha}$ to $\boldsymbol{\alpha}^{\prime}$ and keeps $\Sigma, \boldsymbol{\beta}, \boldsymbol{\gamma}$ and $\mathbf{z}$ unchanged. The 4-manifold $W_{\alpha \beta \gamma}$ is obtained by attaching 3-handles to $W_{\alpha^{\prime} \alpha \beta \gamma}$. Abusing the notation, denote the restriction of $\mathfrak{t}$ to $W_{\alpha^{\prime} \alpha \beta \gamma}$ by $\mathfrak{t}$. Let $\mathfrak{S}_{e}$ be the homomorphism associated with the $\mathbb{A}$-diagram $\left(\Sigma, \boldsymbol{\alpha}^{\prime}, \boldsymbol{\alpha}, \boldsymbol{\beta}, \boldsymbol{\gamma}, \mathfrak{u}, \mathfrak{t}\right)$ and the distinguished generators $\Theta_{\alpha^{\prime} \alpha}$ and $\Theta_{\beta \gamma}$, defined by 
counting holomorphic squares. Considering different possible degenerations of a square either to a bigon and a square or to a pair of triangles gives the relation

$$
\Psi_{e} \circ \mathfrak{f}_{\mathcal{H}}-\mathfrak{f}_{\mathcal{H}^{\prime}} \circ \Phi_{e}=\mathfrak{S}_{e} \circ d+d \circ \mathfrak{S}_{e},
$$

following the standard arguments in Heegaard Floer theory. With a similar argument, if $e$ is a Heegaard move that changes $\boldsymbol{\beta}$ to $\boldsymbol{\beta}^{\prime}$ (respectively $\boldsymbol{\gamma}$ to $\boldsymbol{\gamma}^{\prime}$ ) and keeps $\Sigma, \boldsymbol{\alpha}, \boldsymbol{\gamma}$ (respectively $\boldsymbol{\beta}$ ) and $\mathbf{z}$ fixed, the diagram commutes.

Suppose $e$ changes both $\boldsymbol{\beta}$ and $\boldsymbol{\gamma}$ simultaneously to $\boldsymbol{\beta}^{\prime}$ and $\boldsymbol{\gamma}^{\prime}$. We obtain a pair of maps $\mathfrak{S}_{i}=\mathfrak{S}_{e, i}, i=1,2$ which correspond to the Heegaard quadruples

$$
\left(\Sigma, \boldsymbol{\alpha}, \boldsymbol{\beta}, \boldsymbol{\beta}^{\prime}, \boldsymbol{\gamma}^{\prime}, \mathfrak{u}, \mathfrak{t}\right) \text { and }\left(\Sigma, \boldsymbol{\alpha}, \boldsymbol{\beta}, \boldsymbol{\gamma}, \boldsymbol{\gamma}^{\prime}, \mathfrak{u}, \mathfrak{t}\right),
$$

respectively. Denote the holomorphic triangle maps corresponding to the Heegaard subdiagrams

$$
\left(\Sigma, \boldsymbol{\alpha}, \boldsymbol{\beta}, \boldsymbol{\gamma}^{\prime}, \mathfrak{u}, \mathfrak{t}\right),\left(\Sigma, \boldsymbol{\beta}, \boldsymbol{\gamma}, \boldsymbol{\gamma}^{\prime}, \mathfrak{u}, \mathfrak{t}\right) \text { and }\left(\Sigma, \boldsymbol{\beta}, \boldsymbol{\beta}^{\prime}, \boldsymbol{\gamma}^{\prime}, \mathfrak{u}, \mathfrak{t}\right)
$$

by $\mathfrak{f}_{\alpha \beta \gamma^{\prime}}, \mathfrak{f}_{\beta \gamma \gamma^{\prime}}$ and $\mathfrak{f}_{\beta \beta^{\prime} \gamma^{\prime}}$, respectively.

The images of the distinguished generator $\Theta_{\beta \gamma} \otimes \Theta_{\gamma \gamma^{\prime}}$ under $\mathfrak{f}_{\beta \gamma \gamma^{\prime}}$ and the distinguished generator $\Theta_{\beta \beta^{\prime}} \otimes \Theta_{\beta^{\prime} \gamma^{\prime}}$ under $\mathfrak{f}_{\beta \beta^{\prime} \gamma^{\prime}}$ is the distinguished generator $\Theta_{\beta \gamma^{\prime}}$ which corresponds to $\left(\Sigma, \boldsymbol{\beta}, \boldsymbol{\gamma}^{\prime}, \mathfrak{u}, \mathfrak{t}\right)$. This should be done independently for each one of the Heegaard moves. The proofs follow from the standard arguments in Heegaard Floer theory since the Heegaard triples $\left(\Sigma, \boldsymbol{\beta}, \boldsymbol{\gamma}, \boldsymbol{\gamma}^{\prime}, \mathbf{z}\right)$ and $\left(\Sigma, \boldsymbol{\beta}, \boldsymbol{\beta}^{\prime}, \boldsymbol{\gamma}^{\prime}\right)$ have standard forms.

Let us abuse the notation and denote $\mathfrak{f}_{\alpha \beta \gamma^{\prime}}\left(-\otimes \Theta_{\beta \gamma^{\prime}}\right)$ by $\mathfrak{f}_{\alpha \beta \gamma^{\prime}}$. Setting $\mathfrak{S}_{e}=\mathfrak{S}_{1}+\mathfrak{S}_{2}$, the study of different possible degenerations of a square to a bigon and a square or to two triangles gives

$$
\begin{aligned}
& \mathfrak{f}_{\alpha \beta \gamma^{\prime}}-\mathfrak{f}_{\mathcal{H}^{\prime}} \circ \Phi_{e}=\mathfrak{S}_{1} \circ d+d \circ \mathfrak{S}_{1} \text { and } \\
& \Psi_{e} \circ \mathfrak{f}_{\mathcal{H}}-\mathfrak{f}_{\alpha \beta \gamma^{\prime}}=\mathfrak{S}_{2} \circ d+d \circ \mathfrak{S}_{2} \\
\Rightarrow & \Psi_{e} \circ \mathfrak{f}_{\mathcal{H}}-\mathfrak{f}_{\mathcal{H}^{\prime}} \circ \Phi_{e}=\mathfrak{S}_{e} \circ d+d \circ \mathfrak{S}_{e} .
\end{aligned}
$$

Note that the map $\mathfrak{S}_{e}$ is trivial when $e$ is a stabilization or destabilization, provided that the complex structure is sufficiently stretched along the neck. 


\section{The COBORDISM MAP AND ITS INVARIANCE}

Let $\mathcal{C}=\left[W, F, \mathfrak{t}, \mathfrak{u}_{F}\right]$ be a stable $\mathbb{A}$-cobordism from $\mathcal{T}=[M, T, \mathfrak{s}, \mathfrak{u}]$ to $\mathcal{T}^{\prime}=\left[M^{\prime}, T^{\prime}, \mathfrak{s}^{\prime}, \mathfrak{u}^{\prime}\right]$. Consider an indexed parametrized decomposition

$$
\widetilde{\mathfrak{C}}: \mathcal{W}=\mathcal{W}_{1} \cup_{\left(M_{1}, T_{1}\right)} \mathcal{W}_{2} \cup_{\left(M_{2}, T_{2}\right)} \mathcal{W}_{3}
$$

Recall that for $i=1,3$, the cobordism $\mathcal{W}_{i}$ is parametrized by a set $\mathbb{S}_{i} \subset M_{i-1} \backslash T_{i-1}$ of pairwise disjoint framed $(i-1)$-spheres, and a diffeomorphism

$$
d_{i}:\left(M_{i-1}\left(\mathbb{S}_{i}\right), T_{i-1}\right) \rightarrow\left(M_{i}, T_{i}\right) .
$$

Further, $\mathcal{W}_{2}$ is parametrized by a framed link $\mathbb{S}_{2}$ and a set of pairwise disjoint framed arcs $\mathbb{I}$ in $\left(M_{1}, T_{1}\right)$ along with a diffeomorphism

$$
d_{2}:\left(M_{1}\left(\mathbb{S}_{2}\right), T_{1}(\mathbb{I})\right) \rightarrow\left(M_{2}, T_{2}\right) .
$$

For each $\mathcal{W}_{i}=\left(W_{i}, F_{i}\right)$, let $\mathfrak{t}_{i}$ and $\mathfrak{u}_{i}$ be the $\operatorname{Spin}^{c}$ structure and the $\mathbb{A}$-coloring induced by $\mathfrak{t}$ and $\mathfrak{u}_{F}$, respectively. Note that $\mathfrak{t}$ determines $\mathfrak{t}_{i}$ for $i=1,2,3$, while $\mathfrak{t}_{2}$ determines $\mathfrak{t}$. Let $\mathcal{C}_{i}=$ $\left[W_{i}, F_{i}, \mathfrak{s}_{t} i, \mathfrak{u}_{i}\right]$. In addition, $\mathfrak{t}$ and $\mathfrak{u}_{F}$ induce an $\operatorname{Spin}^{c}$ structure on $\left(M_{i}, T_{i}\right)$, denoted by $\mathfrak{s}_{i}$, and an $\mathbb{A}$-coloring denoted by $\mathfrak{u}_{i}$, abusing the notation. For $0 \leq i \leq 3$, let $\mathcal{T}_{i}=\left[M_{i}, T_{i}, \mathfrak{s}_{i}, \mathfrak{u}_{i}\right]$, where $\mathcal{T}_{0}=\mathcal{T}$, and $\mathcal{T}_{3}=\mathcal{T}^{\prime}$.

For every $\mathbb{A}$-module $\mathbb{M}$, the constructions of Section 5 and Section 6 associate naturally defined $\mathbb{A}$-homomorphisms

$$
\mathfrak{f}_{i}=\operatorname{HF}^{\mathbb{M}}\left(d_{i}\right) \circ \mathfrak{f}_{\mathbb{S}_{i}}^{\mathbb{M}}: \operatorname{HF}^{\mathbb{M}}\left(\mathcal{T}_{i-1}\right) \longrightarrow \operatorname{HF}^{\mathbb{M}}\left(\mathcal{T}_{i}\right)
$$

to the parametrized $\mathbb{A}$-cobordism $\mathcal{C}_{i}$ for $i=1,3$ and

$$
\mathfrak{f}_{2}=\operatorname{HF}^{\mathbb{M}}\left(d_{2}\right) \circ \mathfrak{f}_{\mathcal{C}_{2}, \mathbb{I}, \mathbb{S}_{2}}^{\mathbb{M}}: \operatorname{HF}^{\mathbb{M}}\left(\mathcal{T}_{1}\right) \longrightarrow \operatorname{HF}^{\mathbb{M}}\left(\mathcal{T}_{2}\right)
$$

to the parametrized $\mathbb{A}$-cobordism $\mathcal{C}_{2}$. Subsequently, we may define

$$
\mathfrak{f}_{\mathcal{C}, \widetilde{\mathfrak{C}}}^{\mathbb{M}}: \operatorname{HF}^{\mathbb{M}}(\mathcal{T}) \longrightarrow \operatorname{HF}^{\mathbb{M}}\left(\mathcal{T}^{\prime}\right), \quad \mathfrak{f}_{\mathcal{C}, \widetilde{\mathfrak{C}}}^{\mathbb{M}}=\mathfrak{f}_{3} \circ \mathfrak{f}_{2} \circ \mathfrak{f}_{1}
$$

The homomorphism $\mathfrak{f}_{\mathcal{C}, \widetilde{\mathcal{C}}}^{\mathbb{M}}$ is well-defined and natural, while a priori it depends on the indexed parametrized decomposition $\widetilde{\mathfrak{C}}$.

Theorem 7.1. Let $\mathcal{C}$ be an $\mathbb{A}$-cobordism from the $\mathbb{A}$-tangle $\mathcal{T}$ to the $\mathbb{A}$-tangle $\mathcal{T}^{\prime}$. For every $\mathbb{A}$ module $\mathbb{M}$, the $\mathbb{A}$-homomorphism

$$
\mathfrak{f}_{\mathcal{C}, \widetilde{\mathbb{C}}}^{\mathbb{M}}: \operatorname{HF}^{\mathbb{M}}(\mathcal{T}) \rightarrow \operatorname{HF}^{\mathbb{M}}\left(\mathcal{T}^{\prime}\right)
$$

is an invariant of $\mathcal{C}$. More precisely, this $\mathbb{A}$-homomorphism does not depend on the choice of the indexed parametrized decomposition $\widetilde{\mathfrak{C}}$ for $\mathcal{W}$, which was used in its definition.

Proof. With the above notation fixed, Theorem 4.12 reduces the proof to showing the invariance of the homomorphism $\mathfrak{f}_{\mathcal{C}, \widetilde{\mathfrak{C}}}^{\mathbb{M}}$ under the following changes:

(1) Sliding a component of $\mathbb{S}_{i}$ on another component of $\mathbb{S}_{i}$ for $i=1,2,3$,

(2) Sliding a component of $\mathbb{I}$ on another component of $\mathbb{I}$ or a component of $\mathbb{S}_{2}$,

(3) Sliding a component of $\mathbb{S}_{2}$ on a component of $\mathbb{I}$,

(4) Creation and cancellation of index one/two or two/three critical points,

(5) Diffeomorphism equivalences.

Invariance under the move (1) follows from the arguments in OS06, Subsection 4.4], and invariance under the moves (4) and (5) is straightforward. We will prove invariance under the moves (2) and (3) in Sections 7.1 and 7.2 . 
7.1. Invariance under arc slides. Let $\mathcal{C}=\left[W, F, \mathfrak{t}, \mathfrak{u}_{F}\right]$ be an $\mathbb{A}$-cobordism defined by an acceptable set of framed arcs $\mathbb{I}$ and a framed link $\mathbb{S}$ in an $\mathbb{A}$-tangle $\mathcal{T}=[M, T, \mathfrak{s}, \mathfrak{u}]$. Thus, the $\mathbb{A}$-cobordism $\mathcal{C}$ is from $\mathcal{T}$ to $\mathcal{T}^{\prime}=\left[M(\mathbb{S}), T(\mathbb{I}), \mathfrak{s}^{\prime}, \mathfrak{u}^{\prime}\right]$ where $\mathfrak{s}^{\prime}=\left.\mathfrak{t}\right|_{M(\mathbb{S})}$ and $\mathfrak{u}^{\prime}$ is the $\mathbb{A}$-coloring induced by $\mathfrak{u}_{F}$ on $(M(\mathbb{S}), T(\mathbb{I}))$. Suppose $\widetilde{\mathbb{I}}$ is an acceptable set of framed arcs obtained from $\mathbb{I}$ by arc slides. Corresponding to $(\widetilde{I}, \mathbb{S})$ we get a diffeomorphism

$$
D:(W, F) \rightarrow(\widetilde{W}, \widetilde{F})
$$

where $(\widetilde{W}, \widetilde{F})$ is the cobordism from $(M, T)$ to $(M(\mathbb{S}), T(\widetilde{\mathbb{I}}))$ defined by $(\widetilde{\mathbb{I}}, \mathbb{S})$. Note that $d^{0}=$ $\left.D\right|_{(M, T)}$ is isotopic to identity and let $d=\left.D\right|_{(M(\mathbb{S}), T(\mathbb{I}))}$. So, $(\mathbb{I}, \mathbb{S}, d)$ and $(\widetilde{\mathbb{I}}, \mathbb{S}$, id $)$ are two parameterizations of $(\widetilde{W}, \widetilde{F})$. The $\operatorname{Spin}^{c}$ structure $\mathfrak{t}$ and the $\mathbb{A}$-coloring $\mathfrak{u}_{F}$ induce a Spin ${ }^{c}$ structure and and $\mathbb{A}$-coloring on $(\widetilde{W}, \widetilde{F})$, denoted by $\widetilde{\mathfrak{u}}_{F}$ and $\widetilde{\mathfrak{t}}$. Set $\widetilde{\mathcal{C}}=\left[\widetilde{W}, \widetilde{F}, \widetilde{\mathfrak{t}}, \widetilde{\mathfrak{u}}_{F}\right]$ and let $\mathcal{T}^{\prime \prime}=\left[M(\mathbb{S}), T(\widetilde{\mathbb{I}}), \mathfrak{s}^{\prime \prime}, \mathfrak{u}^{\prime \prime}\right]$ where $\mathfrak{s}^{\prime \prime}$ and $\mathfrak{u}^{\prime \prime}$ are induced by $\widetilde{\mathfrak{u}}_{F}$ and $\widetilde{\mathfrak{t}}$, respectively. Thus, $d$ is a diffeomorphism between the $\mathbb{A}$-tangles $\mathcal{T}^{\prime}$ and $\mathcal{T}^{\prime \prime}$.

Theorem 7.2. With the above notation fixed, for any $\mathbb{A}$-module $\mathbb{M}$

$$
\operatorname{HF}^{\mathbb{M}}(d) \circ \mathfrak{f}_{\mathcal{C}, I, \mathbb{S}}^{\mathbb{M}}=\mathfrak{f}_{\widetilde{\mathcal{C}}, \widetilde{I}, \mathbb{S}}^{\mathbb{M}}: \operatorname{HF}^{\mathbb{M}}(\mathcal{T}) \longrightarrow \operatorname{HF}^{\mathbb{M}}\left(\mathcal{T}^{\prime \prime}\right)
$$

Proof. Assume $\widetilde{\mathbb{I}}$ is obtained from $\mathbb{I}$ by a single arc slide of $\mathbb{I}_{1}$ over $\mathbb{I}_{2}$. Let

$$
\mathcal{H}=\left(\Sigma, \boldsymbol{\alpha}=\left\{\alpha_{1}, \ldots, \alpha_{\ell}\right\}, \boldsymbol{\beta}=\left\{\beta_{1}, \ldots, \beta_{\ell}\right\}, \boldsymbol{\gamma}=\left\{\gamma_{1}, \ldots, \gamma_{\ell}\right\}, \mathfrak{u}: \mathbf{z} \rightarrow \mathbb{A}, \mathfrak{t}\right)
$$

be an $\mathbb{A}$-diagram subordinate to $\mathbb{I}$ and $\mathbb{S}$, as in Definition 6.5. Then, we obtain an $\mathbb{A}$-diagram subordinate to $\widetilde{\mathbb{I}}$ and $\mathbb{S}$ by handle sliding $\beta_{2}$ over $\beta_{1}$ and $\gamma_{1}$ over $\gamma_{2}$ as in Figure 7 . Here, $\beta_{i}$ and $\gamma_{i}$ are the closed curves corresponding to $\mathbb{I}_{i}$ for $i=1,2$. Let

$$
\widetilde{\mathcal{H}}=(\Sigma, \boldsymbol{\alpha}, \widetilde{\boldsymbol{\beta}}, \widetilde{\gamma}, \mathfrak{u}: \mathbf{z} \rightarrow \mathbb{A}, \widetilde{\mathfrak{t}})
$$

be the resulting Heegaard triple. Following an argument analogous to the proof of Theorem 6.9. we show that the diagram

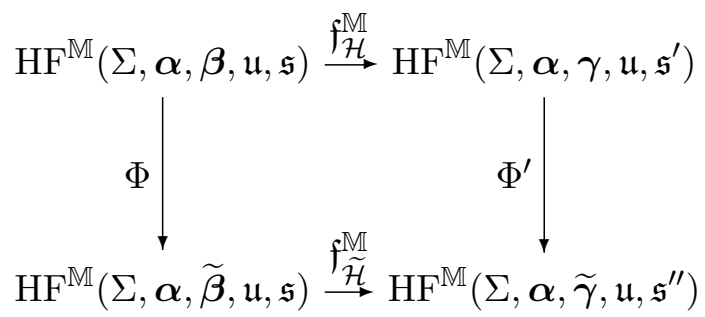

commutes. Here, $\Phi$ and $\Phi^{\prime}$ are isomorphisms corresponding to the aforementioned handle slides.

On the other hand, let $h: \Sigma \rightarrow \Sigma$ be the diffeomorphism, which maps $\beta_{2}$ to $\beta_{1}, \gamma_{1}$ to $\gamma_{2}$ and preserves the rest of $\beta$ and $\gamma$ curves. Then, $h$ induces the diffeomorphism $D$ from $(W, F)$ to $(\widetilde{W}, \widetilde{F})$ as well as the diffeomorphism $d$, and

$$
\widetilde{\mathcal{H}}^{\prime}=(\Sigma, h(\boldsymbol{\alpha}), \widetilde{\boldsymbol{\beta}}, \widetilde{\boldsymbol{\gamma}}, \mathfrak{u}: \mathbf{z} \rightarrow \mathbb{A}, \widetilde{\mathfrak{t}})
$$

is the corresponding Heegaard triple subordinate to $(\widetilde{\mathbb{I}}, \mathbb{S})$ for $(\widetilde{W}, \widetilde{F})$. Note that $\widetilde{\mathcal{H}}^{\prime}$ is obtained from $\widetilde{\mathcal{H}}$ by a sequence $e$ of isotopy and handle slide on $\alpha$ curves i.e. moves of type (1) in Lemma 6.6. Theorem 6.9 implies that $\Psi_{e} \circ \mathfrak{f}_{\widetilde{\mathcal{H}}}=\mathfrak{f}_{\widetilde{\mathcal{H}}^{\prime}} \circ \Phi_{e}$, where

$$
\begin{aligned}
& \Phi_{e}: \operatorname{HF}^{\mathbb{M}}(\Sigma, \boldsymbol{\alpha}, \widetilde{\boldsymbol{\beta}}, \mathfrak{u}, \mathfrak{s}) \rightarrow \operatorname{HF}^{\mathbb{M}}(\Sigma, h(\boldsymbol{\alpha}), \widetilde{\boldsymbol{\beta}}, \mathfrak{u}, \mathfrak{s}) \text { and } \\
& \Psi_{e}: \operatorname{HF}^{\mathbb{M}}\left(\Sigma, \boldsymbol{\alpha}, \widetilde{\boldsymbol{\gamma}}, \mathfrak{u}, \mathfrak{s}^{\prime}\right) \rightarrow \operatorname{HF}^{\mathbb{M}}\left(\Sigma, h(\boldsymbol{\alpha}), \widetilde{\gamma}, \mathfrak{u}, \mathfrak{s}^{\prime \prime}\right)
\end{aligned}
$$

are the isomormphisms associated with the Heegaard moves $e$. Since $d$ is induced by $h$, it follows that,

$$
d_{\star} \circ \mathfrak{f}_{\mathcal{H}}^{\mathbb{M}}=\Psi_{e} \circ \Phi^{\prime} \circ \mathfrak{f}_{\mathcal{H}}^{\mathbb{M}}=\mathfrak{f}_{\widetilde{\mathcal{H}}^{\prime}}^{\mathbb{M}} \circ \Phi_{e} \circ \Phi=\mathfrak{f}_{\widetilde{\mathcal{H}}^{\prime}}^{\mathbb{M}} \circ d_{\star}^{0} .
$$

Therefore, $\operatorname{HF}^{\mathbb{M}}(d) \circ \mathfrak{f}_{\mathcal{C}, \mathbb{I}, \mathbb{S}}^{\mathbb{M}}=\mathfrak{f}_{\widetilde{\mathcal{C}}, \widetilde{I}, \mathbb{S}}^{\mathbb{M}}$. 
We may thus restrict our attention to ordered sets of framed arcs. This is particularly useful when we study the composition law in the following subsections.

7.2. A composition law for framed arcs and links. Let $\mathcal{C}=\left[W, F, \mathfrak{t}, \mathfrak{u}_{F}\right]$ be an $\mathbb{A}$-cobordism from the $\mathbb{A}$-tangle $\mathcal{T}=[M, T, \mathfrak{s}, \mathfrak{u}]$ to the $\mathbb{A}$-tangle $\mathcal{T}^{\prime}=\left[M^{\prime}, T^{\prime}, \mathfrak{s}^{\prime}, \mathfrak{u}^{\prime}\right]$. Further, assume that $(W, F)$ is the cobordism corresponding to an acceptable set of framed arcs $\mathbb{I}$, and a framed link $\mathbb{S}$. Given a decomposition $\mathbb{S}=\mathbb{S}^{1} \amalg \mathbb{S}^{2}$ and $\mathbb{I}=\mathbb{I}^{1} \amalg \mathbb{I}^{2}$ where $\mathbb{I}^{1}$ is acceptable, we obtain a decomposition

$$
(W, F)=\left(W_{1}, F_{1}\right) \cup_{\left(M^{\prime \prime}, T^{\prime \prime}\right)}\left(W_{2}, F_{2}\right) .
$$

Here, $\left(W_{1}, F_{1}\right)$ is the cobordism associated with $\left(\mathbb{I}^{1}, \mathbb{S}^{1}\right)$ and $\left(W_{2}, F_{2}\right)$ is the cobordism associated with $\left(\mathbb{I}^{2}, \mathbb{S}^{2}\right)$ in $\left(M^{\prime \prime}, T^{\prime \prime}\right)=\left(M\left(\mathbb{S}^{1}\right), T\left(\mathbb{I}^{1}\right)\right)$. For $i=1,2$, let $\mathfrak{u}_{i}$ denote the $\mathbb{A}$-coloring induced by $\mathfrak{u}_{F}$ on $\left(W_{i}, F_{i}\right)$, and $\mathfrak{t}_{i}=\left.\mathfrak{t}\right|_{W_{i}}$. Consider the corresponding $\mathbb{A}$-cobordisms

$$
\mathcal{C}^{1}=\left[W_{1}, F_{1}, \mathfrak{t}_{1}, \mathfrak{u}_{1}\right]: \mathcal{T} \leadsto \mathcal{T}^{\prime \prime} \quad \text { and } \quad \mathcal{C}^{2}=\left[W_{2}, F_{2}, \mathfrak{t}_{2}, \mathfrak{u}_{2}\right]: \mathcal{T}^{\prime \prime} \leadsto \mathcal{T}^{\prime},
$$

where $\mathcal{T}^{\prime \prime}$ denotes the $\mathbb{A}$-tangle obtained by equipping $\left(M^{\prime \prime}, T^{\prime \prime}\right)$ with the induced $\mathbb{A}$-coloring and Spin $^{c}$ structure from $\mathfrak{u}_{F}$ and $\mathfrak{t}$, respectively.

The restrictions of $\mathfrak{t}$ to $W_{1}$ and $W_{2}$ remain unchanged, under modifying $\mathfrak{t}$ by adding an element of $\delta H^{1}\left(M^{\prime \prime}, \mathbb{Z}\right)$, where $\delta: H^{1}\left(M^{\prime \prime}, \mathbb{Z}\right) \rightarrow H^{2}(W, \mathbb{Z})$ is the connecting homomorphism in the MayerVietoris sequence for $\left(W_{1}, W_{2}\right)$. So, let $\mathfrak{T}$ be the set of all $\operatorname{Spin}^{c}$ classes on $W$ so that their restrictions to $W_{1}$ and $W_{2}$ are equal to $\mathfrak{t}_{1}$ and $\mathfrak{t}_{2}$, respectively.

Theorem 7.3. With the above notation fixed, for every $\mathbb{A}$-module $\mathbb{M}$ we have

$$
\mathfrak{f}_{\mathcal{C}^{2}, \mathbb{I}^{2}, \mathbb{S}^{2}}^{\mathbb{M}} \circ \mathfrak{f}_{\mathcal{C}^{1}, \mathbb{I}^{1}, \mathbb{S}^{1}}^{\mathbb{M}}=\sum_{\overline{\mathfrak{t}} \in \mathfrak{T}} \mathfrak{f}_{\mathcal{C}(\mathfrak{t}), \mathbb{I}, \mathbb{S}}^{\mathbb{M}}
$$

where $\mathcal{C}(\overline{\mathfrak{t}})$ is the $\mathbb{A}$-cobordism obtained from $\mathcal{C}$ by replacing $\mathfrak{t}$ with $\overline{\mathfrak{t}}$.
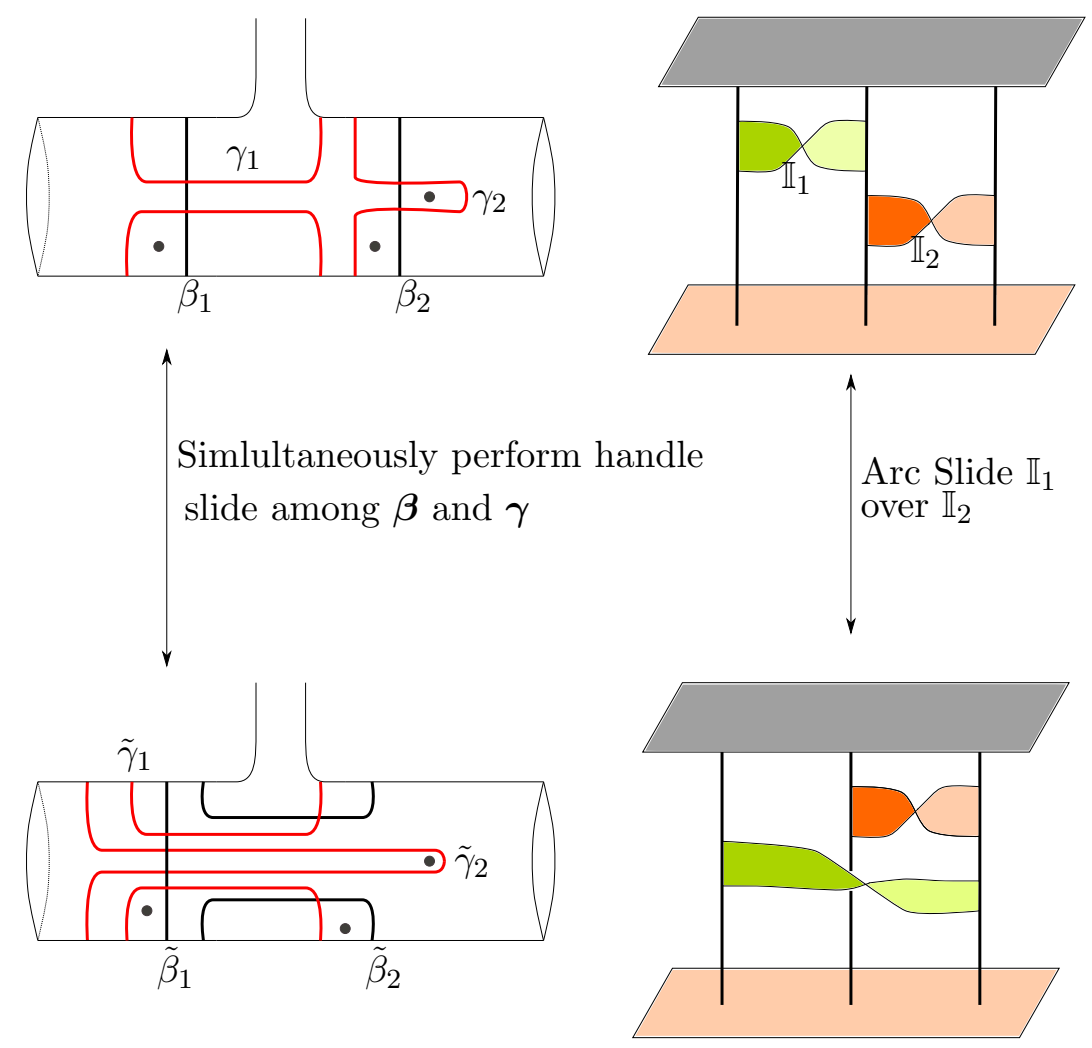

FIGURE 7 . Sliding a framed arc $\mathbb{I}_{1}$ over another framed arc $\mathbb{I}_{2}$. 
Proof. Any acceptable set of framed arcs can be turned into an ordered set by arc slides. So Theorem 7.2 implies that without loss of generality, we may assume $\mathbb{I}$ is ordered such that

$$
\mathbb{I}^{1}=\left(\mathbb{I}_{1}, \ldots, \mathbb{I}_{n_{1}}\right) \quad \text { and } \quad \mathbb{I}^{2}=\left(\mathbb{I}_{n_{1}+1}, \ldots, \mathbb{I}_{n_{1}+n_{2}}\right), \quad \text { where } n_{1}+n_{2}=n .
$$

Furthermore, suppose that

$$
\mathbb{S}^{1}=\left(\mathbb{S}_{1}, \ldots, \mathbb{S}_{m_{1}}\right) \quad \text { and } \quad \mathbb{S}^{2}=\left(\mathbb{S}_{m_{1}+1}, \ldots, \mathbb{S}_{m=m_{1}+m_{2}}\right) .
$$

Let us fix an $\mathbb{A}$-diagram $\mathcal{H}=(\Sigma, \boldsymbol{\alpha}, \boldsymbol{\beta}, \boldsymbol{\gamma}, \mathfrak{u}, \mathfrak{t})$ subordinate to $(\mathbb{I}, \mathbb{S})$. Let $\boldsymbol{\delta}=\left\{\delta_{1}, \ldots, \delta_{\ell}\right\}$ denote a set of curves obtained as follows:

- Let $\delta_{i}$ be a Hamiltonian isotope of $\gamma_{i}$ for every $i$ in

$$
A=\left\{1, \ldots, n_{1}\right\} \cup\left\{n+1, \ldots, n+m_{1}\right\} .
$$

- For every $i \in\{1, \ldots, \ell\}-A$, let $\delta_{i}$ be a Hamiltonian isotope of $\beta_{i}$.

The $\operatorname{Spin}^{c}$ structures $\mathfrak{t}_{1}$ and $\mathfrak{t}_{2}$ induce $\operatorname{Spin}^{c}$ structures on $W_{\alpha \beta \delta}$ and $W_{\alpha \delta \gamma}$, which will also be denoted by $\mathfrak{t}_{1}$ and $\mathfrak{t}_{2}$, by slight abuse of notation. Then the $\mathbb{A}$-diagrams

$$
\mathcal{H}_{1}=\left(\Sigma, \boldsymbol{\alpha}, \boldsymbol{\beta}, \boldsymbol{\delta}, \mathfrak{u}, \mathfrak{t}_{1}\right) \text { and } \mathcal{H}_{2}=\left(\Sigma, \boldsymbol{\alpha}, \boldsymbol{\delta}, \boldsymbol{\gamma}, \mathfrak{u}, \mathfrak{t}_{2}\right)
$$

are subordinate to $\left(\mathbb{I}^{1}, \mathbb{S}^{1}\right)$ and $\left(\mathbb{I}^{2}, \mathbb{S}^{2}\right)$ and correspond to $\mathcal{C}_{1}$ and $\mathcal{C}_{2}$, respectively. These two Heegaard triples determine the maps $\mathfrak{f}_{1}=\mathfrak{f}_{\mathcal{C}^{1}, \mathbb{I}^{1}, \mathbb{S}^{1}}$ and $\mathfrak{f}_{2}=\mathfrak{f}_{\mathcal{C}^{2}, \mathbb{I}^{2}, \mathbb{S}^{2}}$. Furthermore, the $\mathbb{A}$-diagram

$$
(\Sigma, \boldsymbol{\alpha}, \boldsymbol{\beta}, \boldsymbol{\delta}, \boldsymbol{\gamma}, \mathfrak{u}, \mathfrak{T})
$$

and the distinguished generators

$$
\Theta_{\beta \delta} \in \operatorname{HF}\left(\Sigma, \boldsymbol{\beta}, \boldsymbol{\delta}, \mathfrak{u},\left.\mathfrak{t}_{1}\right|_{M_{\beta \delta}}=\mathfrak{s}_{0}\right) \quad \text { and } \quad \Theta_{\delta \gamma} \in \operatorname{HF}\left(\Sigma, \boldsymbol{\delta}, \boldsymbol{\gamma}, \mathfrak{u},\left.\mathfrak{t}_{2}\right|_{M_{\delta \gamma}}=\mathfrak{s}_{0}\right)
$$

determine a holomorphic square map

$$
\mathfrak{S}: \operatorname{HF}(\Sigma, \boldsymbol{\alpha}, \boldsymbol{\beta}, \mathfrak{u}, \mathfrak{s})=\operatorname{HF}(\mathcal{T}) \longrightarrow \operatorname{HF}\left(\Sigma, \boldsymbol{\alpha}, \boldsymbol{\gamma}, \mathfrak{u}, \mathfrak{s}^{\prime}\right)=\operatorname{HF}\left(\mathcal{T}^{\prime}\right)
$$

Considering different possible degenerations of a square class of index 0 and applying a mild generalization of [OS04c, Theorem 8.16], we obtain the relation

$$
\mathfrak{f}_{2} \circ \mathfrak{f}_{1}-\sum_{\overline{\mathfrak{t}} \in \mathfrak{T}} \mathfrak{f}_{\alpha \beta \gamma, \overline{\mathfrak{t}}}\left(-\otimes \mathfrak{f}_{\beta \delta \gamma}\left(\Theta_{\beta \delta} \otimes \Theta_{\delta \gamma}\right)\right)=\mathfrak{S} \circ d+d \circ \mathfrak{S} .
$$

Here $\mathfrak{f}_{\alpha \beta \gamma, \overline{\mathfrak{t}}}$ and $\mathfrak{f}_{\beta \delta \gamma}$ are the holomorphic triangle maps associated with the Heegaard triples

$$
(\Sigma, \boldsymbol{\alpha}, \boldsymbol{\beta}, \boldsymbol{\gamma}, \mathfrak{u}, \overline{\mathfrak{t}}) \text { and }\left(\Sigma, \boldsymbol{\beta}, \boldsymbol{\delta}, \boldsymbol{\gamma}, \mathfrak{u}, \mathfrak{t}_{0}\right),
$$

respectively, and $\mathfrak{t}_{0}$ denotes a canonically determined $\operatorname{Spin}^{c}$ structure on the 4-manifold $W_{\beta \delta \gamma}$. In order to complete the proof, it thus suffices to show that

$$
\mathfrak{f}_{\beta \delta \gamma}\left(\Theta_{\beta \delta} \otimes \Theta_{\delta \gamma}\right)=\Theta_{\beta \gamma} .
$$

Since $(\Sigma, \boldsymbol{\alpha}, \boldsymbol{\beta}, \boldsymbol{\gamma}, \mathbf{z})$ is a Heegaard triple subordinate to $(\mathbb{I}, \mathbb{S})$, the proof of Proposition 6.5 implies the existence of a labeling for the intersection points of $\beta_{i}$ and $\gamma_{i}$ by $d_{i}^{+}$and $d_{i}^{-}$, for $i \in\{1, \ldots, \ell\} \backslash$ $\{n+1, \ldots, n+m\}$, such that $\Theta_{\beta \gamma}$ is represented by

$$
\theta_{\beta \gamma}^{+}=\left\{d_{1}^{+}, \ldots, d_{\ell}^{+}\right\}
$$

Here, $d_{i}^{+}$denotes the only intersection point of $\beta_{i}$ and $\gamma_{i}$ for $n+1 \leq i \leq n+m$. Similarly, it follows from the definition of $\boldsymbol{\delta}$ that we may label the intersection points of $\boldsymbol{\delta}$ with $\boldsymbol{\beta}$ and $\boldsymbol{\gamma}$ such that $\Theta_{\beta \delta}$ and $\Theta_{\delta \gamma}$ are respectively represented by:

$$
\theta_{\beta \delta}^{+}=\left\{c_{1}^{+}, \ldots, c_{\ell}^{+}\right\} \quad \text { and } \quad \theta_{\delta \gamma}^{+}=\left\{b_{1}^{+}, \ldots, b_{\ell}^{+}\right\} .
$$

Let us now assume that $\theta \in \mathbb{T}_{\beta} \cap \mathbb{T}_{\gamma}$ contributes to $\mathfrak{f}_{\beta \gamma \delta}\left(\Theta_{\beta \delta} \otimes \Theta_{\delta \gamma}\right)$ through a triangle class

$$
\Delta=\Delta^{\ell} \in \pi_{2}\left(\theta_{\beta \delta}^{+}, \theta_{\delta \gamma}^{+}, \theta\right)
$$

of Maslov index 0. For any $1 \leq i \leq \ell$, consider the Heegaard triple

$$
H^{i}=\left(\Sigma^{i}=\Sigma\left[\boldsymbol{\beta}_{i+1}, \ldots, \boldsymbol{\beta}_{\ell}\right], \boldsymbol{\beta}^{i}=\left\{\beta_{1}, \ldots, \beta_{i}\right\}, \boldsymbol{\delta}^{i}=\left\{\delta_{1}, \ldots, \delta_{i}\right\}, \gamma^{i}=\left\{\gamma_{1}, \ldots, \gamma_{i}\right\}, \mathbf{z}\right)
$$


where $\Sigma\left[\boldsymbol{\beta}_{i+1}, \ldots, \boldsymbol{\beta}_{\ell}\right]$ denotes the surface obtained from $\Sigma$ by performing surgery along the $\beta$ curves $\left\{\beta_{i+1}, \beta_{i+2}, \ldots, \beta_{\ell}\right\}$. Therefore, $\Sigma^{\ell}$ is obtained from $\Sigma^{\ell-1}$ by attaching a 1-handle, and corresponding to $\Delta^{\ell}$, when the necks are sufficiently stretched, we obtain a triangles class $\Delta^{\ell-1}$ on $\Sigma^{\ell-1}$ and a class $\Delta^{\prime}$ on the attached one-handle which connects

$$
c_{\ell}^{+} \in \beta_{\ell} \cap \delta_{\ell}, \quad b_{\ell}^{+} \in \delta_{\ell} \cap \gamma_{\ell} \quad \text { and } \quad d_{\ell}^{\star} \in \beta_{\ell} \cap \gamma_{\ell},
$$

where $\star$ is either + or - . It also follows that

$$
\mu\left(\Delta^{\ell}\right)=\mu\left(\Delta^{\ell-1}\right)-\epsilon\left(d_{\ell}^{\star}\right),
$$

where $\epsilon\left(d_{\ell}^{+}\right)=0$ and $\epsilon\left(d_{\ell}^{-}\right)=1$. We are thus forced to have $\star=+$. It also follows from the argument of Proposition 5.1 and the second part of Lemma 5.3 that, if the the necks are sufficiently stretched, $\mathcal{M}\left(\Delta^{\ell}\right)$ may be identified with $\mathcal{M}\left(\Delta^{\ell-1}\right)$. This argument may in fact be repeated again and again to show that the generator $\theta$ uses the intersection points

$$
d_{i}^{+} \in \beta_{i} \cap \gamma_{i}, \quad \text { for } i=n+m+1, \ldots, \ell,
$$

and that $\mathcal{M}\left(\Delta^{\ell}\right)$ may be identified with $\mathcal{M}\left(\Delta^{n+m}\right)$, for a triangle class over $\Sigma^{n+m}$.

Now $\Sigma^{n+m}=\Sigma^{n+m-1} \# E$ where $E$ is a surface of genus 1 . If we stretch the connected sum neck, the complex structure on $\Sigma^{n+m}$ converges to the join of complex structures on the $\Sigma^{n+m-1}-$ $\{w\}$ and $E-\left\{w^{\prime}\right\}$. We also obtain a decomposition of $\Delta^{n+m}$ to the triangle classes $\Delta^{n+m-1}$ (on $\Sigma^{n+m-1}$ ) and $\Delta^{\prime}$ (on $E$ ). The generator $\theta$ is forced to use the unique intersection point $d_{n+m} \in \beta_{n+m} \cap \gamma_{n+m}$. From the choice of the intersection points in $\theta_{\beta \delta}^{+}$and $\theta_{\delta \gamma}^{+}$it also follows that $\mu\left(\Delta^{n+m-1}\right)=\mu\left(\Delta^{n+m}\right)=0$. Furthermore, as the weak limit of a sequence of holomorphic curves in $\mathcal{M}\left(\Delta^{n+m}\right)$ as the neck is stretched, we obtain a degenerate holomorphic curve $u^{n+m-1}$ in the 0 -dimensional moduli space $\mathcal{M}\left(\Delta^{n+m-1}\right)$, which has coefficient $k$ at $w$. The holomorphic curve $u^{n+m-1}$ determines a point $\rho\left(u^{n+m-1}\right)$ in $\operatorname{Sym}^{k}(\mathbb{D})$. Let $\Delta_{k}$ denote the union of all triangle classes $\Delta^{\prime}$ over the genus-one surface $E$ with coefficient $k$ over the marked point $w^{\prime}$. The arguments of Section 12 of [Lip06], and in particular Lemma 12.2, Proposition 12.4 (in fact, Proposition A.3), imply that in the aforementioned weak limit, $u^{n+m-1}$ is paired with a degenerate curve $v$ on $E$, which is the union of

$$
E \times \rho\left(u^{n+m-1}\right) \subset E \times \mathbb{D}
$$

and the unique holomorphic representative of $\Delta_{0}$, to produce the only possible curve in the weak limit. Every such weak limit may be perturbed to a holomorphic curve representing $\Delta^{n+m}$, giving an identification of $\mathcal{M}\left(\Delta^{n+m}\right)$ with $\mathcal{M}\left(\Delta^{n+m-1}\right)$, if the connected sum neck is sufficiently long. Again, we may repeat the above argument to find an identification of $\mathcal{M}\left(\Delta^{\ell}\right)$ with $\mathcal{M}\left(\Delta^{n}\right)$, provided that attaching the 1-handles and taking connected sum with surfaces of genus 1 is done using sufficiently stretched necks.

The surface $\Sigma^{n}$ is obtained from $\Sigma^{n-1}$ by attaching a 1-handle and moving two of the markings (which we denote by $z, z^{\prime} \in \mathbf{z}$ ) over the attached 1-handle. The curves $\beta_{n}, \delta_{n}$ and $\gamma_{n}$ are all isotopic to the belt circle of the attached 1-handle, while the positions of $z$ and $z^{\prime}$ in the cylinder representing the neck is the position illustrated on the right-hand-side of Figure 8 . Correspondingly, when the necks are sufficiently stretched, we obtain a triangles class $\Delta^{n-1}$ on $\Sigma^{n-1}$ and a class $\Delta^{\prime}$ on the attached one-handle which connects the intersection points

$$
c_{n}^{+} \in \beta_{n} \cap \delta_{n}, \quad b_{n}^{+} \in \delta_{n} \cap \gamma_{n} \quad \text { and } \quad d_{n}^{\star} \in \beta_{n} \cap \gamma_{n},
$$

where $\star$ is either + or - . It also follows that

$$
\mu\left(\Delta^{n}\right)=\mu\left(\Delta^{n-1}\right)-\epsilon\left(d_{n}^{\star}\right),
$$

where $\epsilon\left(d_{n}^{+}\right)=0$ and $\epsilon\left(d_{n}^{-}\right)=1$. We are thus forced to have $d_{n}=d_{n}^{+}$. It also follows from the argument of Proposition 5.1 and the second part of Lemma 5.3 that, if the the necks are sufficiently stretched, $\mathcal{M}\left(\Delta^{n}\right)$ may be identified with $\mathcal{M}\left(\Delta^{n-1}\right)$. This argument may be repeated to show that the generator $\theta$ uses the intersection points

$$
d_{i}^{+} \in \beta_{i} \cap \gamma_{i}, \quad \text { for } i=1, \ldots, n,
$$




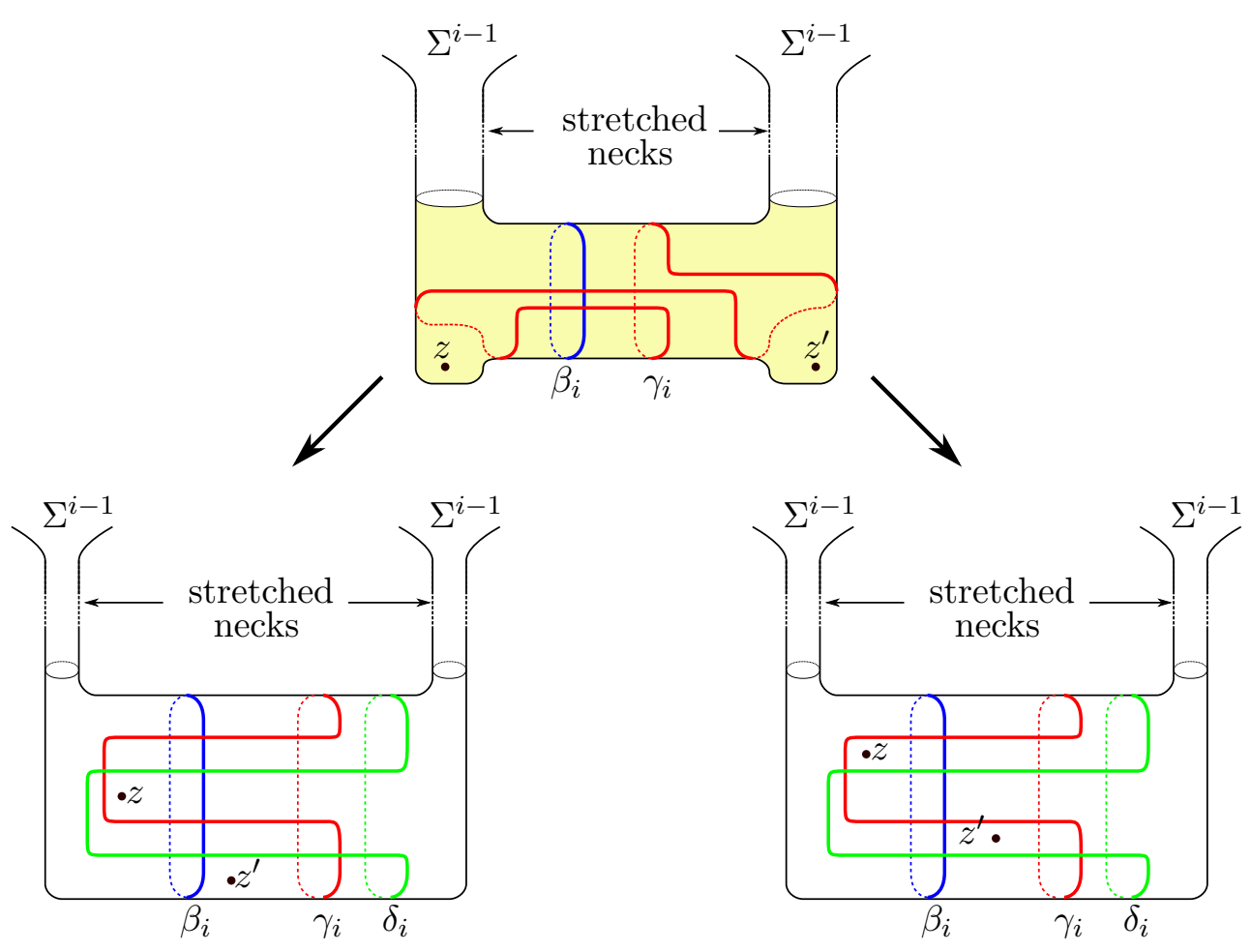

FiguRE 8. As we stretch the neck, $\Sigma^{i}$ degenerates to a subsurface $\Sigma^{i-1}$ and a sphere, which are attached by two long necks. When $i \in\left\{1, \ldots, n_{1}\right\}$ a pair of markings $z, z^{\prime} \in \mathbf{z}$ will land on the sphere as illustrated on the left-hand-side, while for $i \in\left\{n_{1}+1, \ldots, n\right\}$ their location follows the pattern illustrated on the righthand-side.

and that $\mathcal{M}\left(\Delta^{\ell}\right)$ may be identified with $\mathcal{M}\left(\Delta^{1}\right)$, which consists of a single point.

The above argument shows that $\theta$ is forced to represent the generator $\Theta_{\beta \gamma}$, and that the total contribution of holomorphic triangles to the coefficient of $\theta$ in $\mathfrak{f}_{\beta \gamma \delta}\left(\Theta_{\beta \delta} \otimes \Theta_{\delta \gamma}\right)$ is 1. This completes the proof of the theorem.

Corollary 7.4. Assume $\mathcal{C}=\left[W, F, \mathfrak{t}, \mathfrak{u}_{F}\right]$ is an $\mathbb{A}$-cobordism from $\mathcal{T}$ to $\mathcal{T}^{\prime}$, determined by an acceptable set of framed arcs $\mathbb{I}$ and a framed link $\mathbb{S}$ i.e. (II, $\mathbb{S}$, id) gives a parametrization of $(W, F)$. Let $\mathbb{I}^{\prime}$ be an acceptable set of framed arcs obtained from $\mathbb{I}$ by sliding some of its components over some components of $\mathbb{S}$, and similarly $\mathbb{S}^{\prime}$ be the framed link obtained from $\mathbb{S}$ by sliding some of its components over some of the framed arcs in $\mathbb{I}$. Denote the induced diffeomorphisms by

$$
d_{\mathbb{I}}: \mathcal{T}\left(\mathbb{I}^{\prime}, \mathbb{S}\right) \rightarrow \mathcal{T}^{\prime} \quad \text { and } \quad d_{\mathbb{S}}: \mathcal{T}\left(\mathbb{I}, \mathbb{S}^{\prime}\right) \rightarrow \mathcal{T}^{\prime} .
$$

Then for any $\mathbb{A}$-module $\mathbb{M}$ we have

$$
\mathfrak{f}_{\mathcal{C}, \mathbb{I}, \mathbb{S}}^{\mathbb{M}}=\operatorname{HF}^{\mathbb{M}}\left(d_{\mathbb{I}}\right) \circ \mathfrak{f}_{\mathcal{C}, \mathbb{I}^{\prime}, \mathbb{S}}^{\mathbb{M}} \quad \text { and } \quad \mathfrak{f}_{\mathcal{C}, \mathbb{I}, \mathbb{S}}^{\mathbb{M}}=\operatorname{HF}^{\mathbb{M}}\left(d_{\mathbb{S}}\right) \circ \mathfrak{f}_{\mathcal{C}, \mathbb{I}, \mathbb{S}^{\prime}}^{\mathbb{M}}
$$

Proof. Note that $\left(\mathbb{I}^{\prime}, \mathbb{S}, d_{\mathbb{I}}\right)$ and $\left(\mathbb{I}, \mathbb{S}^{\prime}, d_{\mathbb{S}}\right)$ are parametrizations of $\mathcal{C}$, or $\left.(W, F)\right)$. The claim is then a straightforward result of Theorems 7.2 and 7.3

This completes the proof of Theorem 7.1 , and we may now denote the $\mathbb{A}$-homomorphism associated with the $\mathbb{A}$-cobordism $\mathcal{C}$ and the $\mathbb{A}$-module $\mathbb{M}$ by $\mathfrak{f}_{\mathcal{C}}^{\mathbb{M}}$.

7.3. The composition law. In this subsection, we prove a generalization of Theorem 7.3 .

Theorem 7.5. Suppose that $\mathcal{C}_{i}=\left[W_{i}, F_{i}, \mathfrak{t}_{i}, \mathfrak{u}_{i}\right]: \mathcal{T}_{i-1} \rightarrow \mathcal{T}_{i}$ are $\mathbb{A}$-cobordisms for $i=1, \ldots, m$. Let $\mathcal{W}=(W, F)$ be the stable cobordism obtained by putting $\mathcal{W}_{i}=\left(W_{i}, F_{i}\right)$ together and $\mathfrak{u}: \pi_{0}(F) \rightarrow \mathbb{A}$ 
denote the $\mathbb{A}$-coloring induced by $\mathfrak{u}_{i}$. For every $\mathfrak{t} \in \operatorname{Spin}^{c}(W)$ with $\left.\mathfrak{t}\right|_{W_{i}}=\mathfrak{t}_{i}$, let $\mathcal{C}(\mathfrak{t})=[W, F, \mathfrak{t}, \mathfrak{u}]$. Then

$$
\sum_{\substack{\left.\mathfrak{t} \in \operatorname{Spin}^{c}(W) \\ \mathfrak{t}\right|_{W_{i}}=\mathfrak{t}_{i}}} \mathfrak{f}_{\mathcal{C}(\mathfrak{t})}^{\mathbb{M}}=\mathfrak{f}_{\mathcal{C}_{m}}^{\mathbb{M}} \circ \cdots \circ \mathfrak{f}_{\mathcal{C}_{1}}^{\mathbb{M}}
$$

Proof. The definition of the cobordism invariants and Theorem 7.3 reduce the proof of Theorem 7.5 to the case where $m=2$, both $\mathcal{C}_{1}$ and $\mathcal{C}_{2}$ are parametrized elementary $\mathbb{A}$-cobordisms, and one of the following happens.

(1) $\mathcal{W}_{1}$ corresponds to a framed 2 -sphere $\mathbb{S}_{1}$, and $\mathcal{W}_{2}$ corresponds to a framed 0 -sphere $\mathbb{S}_{2}$.

(2) $\mathcal{W}_{1}$ corresponds to a framed 2 -sphere $\mathbb{S}_{1}$, and $\mathcal{W}_{2}$ corresponds to a framed knot $\mathbb{S}_{2}$.

(3) $\mathcal{W}_{1}$ corresponds to a framed 2 -sphere $\mathbb{S}$, and $\mathcal{W}_{2}$ corresponds to a framed arc $\mathbb{I}$.

(4) $\mathcal{W}_{1}$ corresponds to a framed knot $\mathbb{S}_{1}$, and $\mathcal{W}_{2}$ corresponds to a framed 0-sphere $\mathbb{S}_{2}$.

(5) $\mathcal{W}_{1}$ corresponds to a framed arc $\mathbb{I}$, and $\mathcal{W}_{2}$ corresponds to a framed 0 -sphere $\mathbb{S}$.

For all cases, there is a unique $\operatorname{Spin}^{c}$ class $\mathfrak{t}$ on $W$ such that $\mathfrak{t}_{W_{i}}=\mathfrak{t}_{i}$ for $i=1,2$. We may thus set $\mathcal{C}=\mathcal{C}(\mathfrak{t})$. Let $\mathcal{T}=\mathcal{T}_{0}=[M, T, \mathfrak{s}, \mathfrak{u}]$ and $\mathcal{T}^{\prime}=\mathcal{T}_{2}=\left[M^{\prime}, T^{\prime}, \mathfrak{s}^{\prime}, \mathfrak{u}^{\prime}\right]$

In the first case, $W_{1}$ is determined by the framed 2 -sphere $\mathbb{S}_{1}$, so the underlying tangle of $\mathcal{T}_{1}$ is $\left(M_{1}, T_{1}\right)=\left(M\left(\mathbb{S}_{1}\right), T\right)$. Considering this identification, we may assume that $\mathbb{S}_{2}$ lies in $M \backslash$ $\mathbb{S}_{1}=M \cap M_{1}$. Thus, $\mathbb{S}_{2}$ specifies a cobordism $\mathcal{W}_{1}^{r}$ from $(M, T)$ to $\left(M\left(\mathbb{S}_{2}\right), T\right)$. Similarly, $\mathbb{S}_{1}$ specifies a cobordism $\mathcal{W}_{2}^{r}$ from $\left(M\left(\mathbb{S}_{2}\right), T\right)$ to $\left(M^{\prime}, T^{\prime}\right)$. As a result we get an indexed parametrized decomposition $\mathcal{W}_{1}^{r} \cup_{\left(M\left(\mathbb{S}_{2}\right), T\right)} \mathcal{W}_{2}^{r}$ for $(W, F)$.

The $\mathbb{A}$-coloring $\mathfrak{u}$ and the $\operatorname{Spin}^{c}$ class $\mathfrak{t}$ make $\mathcal{W}_{1}^{r}$ and $\mathcal{W}_{2}^{r}$ into $\mathbb{A}$-cobordisms, denoted by $\mathcal{C}_{1}^{r}$ and $\mathcal{C}_{2}^{r}$, respectively. Let $\mathcal{T}^{r}=\left[M\left(\mathbb{S}_{2}\right), T, \mathfrak{s}^{r}=\left.\mathfrak{t}\right|_{M\left(\mathbb{S}_{2}\right)}, \mathfrak{u}\right]$. Consider the following diagram:

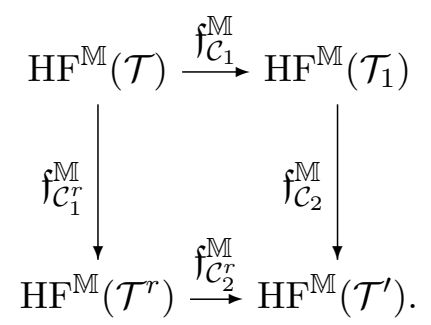

Every generator of $\mathrm{CF}^{\mathbb{M}}(\mathcal{T})$ is of the form $\mathbf{x} \times \theta$, where $\mathbf{x}$ is a generator of the chain complex $\mathrm{CF}^{\mathbb{M}}\left(\mathcal{T}_{1}\right)$ and $\theta$ is one of the two intersection points $\theta_{\alpha \beta}^{1}$ and $\theta_{\beta \alpha}^{1}$ corresponding to the framed 2 -sphere $\mathbb{S}_{1}$, (see Section 5.3). If the necks in the corresponding Heegaard diagrams for $\mathbb{S}_{1}$ and $\mathbb{S}_{2}$ are sufficiently stretched, this generator is mapped to $\mathbf{x} \times \theta \times \theta_{\alpha \beta}^{2}$ under $\mathfrak{f}_{\mathcal{C}_{1}^{r}}^{\mathbb{M}}$. Here $\theta_{\alpha \beta}^{2}$ and $\theta_{\beta \alpha}^{2}$ are the two intersection points which correspond to the 1-handle, attached to $\mathbb{S}_{2}$. The generator $\mathbf{x} \times \theta \times \theta_{\alpha \beta}^{2}$ goes to zero under $\mathfrak{f}_{\mathcal{C}_{2}^{r}}^{\mathbb{M}}$ unless $\theta=\theta_{\beta \alpha}^{1}$, when it is mapped to $\mathbf{x} \times \theta_{\alpha \beta}^{2}$. On the other hand, the image of $\mathbf{x} \times \theta$ under $\mathfrak{f}_{\mathcal{C}_{1}}^{\mathbb{M}}$ is zero unless $\theta=\theta_{\beta \alpha}^{1}$, when

$$
\mathfrak{f}_{\mathcal{C}_{1}}^{\mathbb{M}}\left(\mathbf{x} \times \theta_{\beta \alpha}^{1}\right)=\mathbf{x} \quad \text { and } \quad \mathfrak{f}_{\mathcal{C}_{2}}^{\mathbb{M}}\left(\mathfrak{f}_{\mathcal{C}_{1}}^{\mathbb{M}}\left(\mathbf{x} \times \theta_{\beta \alpha}^{1}\right)\right)=\mathbf{x} \times \theta_{\alpha \beta}^{2}
$$

This implies that the above diagram is commutative and

$$
\mathfrak{f}_{\mathcal{C}_{2}}^{\mathbb{M}} \circ \mathfrak{f}_{\mathcal{C}_{1}}^{\mathbb{M}}=\mathfrak{f}_{\mathcal{C}_{2}^{r}}^{\mathbb{M}} \circ \mathfrak{f}_{\mathcal{C}_{1}^{r}}^{\mathbb{M}}=\mathfrak{f}_{\mathcal{C}}^{\mathbb{M}}
$$

A similar modification to the proofs of [OS06, Proposition 4.19 and Proposition 4.18], imply the heorem in the second and fourth cases, respectively. The only remaining cases are thus the third and the fifth cases.

In the third case, $\mathcal{C}_{1}$ corresponds to attaching a 3 -handle along $\mathbb{S}$ and $\mathcal{C}_{2}$ corresponds to a framed $\operatorname{arc} \mathbb{I}$. This framed arc may clearly be isotoped to $M \backslash \mathbb{S}$. Therefore, we get an indexed parametrized decomposition of $(W, F)$ by switching the order of attached handles. More precisely, let $\mathcal{W}_{1}^{r}$ to be the cobordism determined by $\mathbb{I}$ from $(M, T)$ to $(M, T(\mathbb{I}))$ and $\mathcal{C}_{2}^{r}$ be the cobordism determined by $\mathbb{S}$ from $(M, T(\mathbb{I}))$ to $\left(M^{\prime}, T^{\prime}\right)$. For $i=1,2$, the $\mathbb{A}$-cobordism obtained by equipping $\mathcal{W}_{i}^{r}$ with the 
$\mathbb{A}$-coloring induced by $\mathfrak{u}$ and $\operatorname{Spin}^{c}$ structure induced by $\mathfrak{t}$ is denoted by $\mathcal{C}_{i}^{r}$. Correspondingly, we may choose a Heegaard surface $\Sigma$ in $M$ with the following properties:

- The intersection of $\Sigma$ with $\mathbb{S}$ is an annulus $A$, such that the surface $\Sigma^{\circ}$ in $M_{1}$ obtained by cutting off $A$ and gluing in a pair of disks to the two boundary components, is a Heegaard surface for $M_{1}$. Here, $M_{1}$ is the underlying 3-manifold of $\mathcal{T}_{1}$.

- Each component of $T$ intersects $\Sigma$ transversely in exactly one point. We let $\mathbf{z}=T \cap \Sigma$.

- There are two collections $\boldsymbol{\alpha}$ and $\boldsymbol{\beta}$ of attaching circles on $\Sigma$ such that $\boldsymbol{\alpha} \cap A$ is a single circle $\alpha$ which is a small Hamiltonian isotope of the circle $\{\beta\}=\boldsymbol{\beta} \cap A$. Moreover, the diagram $(\Sigma, \boldsymbol{\alpha}, \boldsymbol{\beta}, \mathfrak{u}: \mathbf{z} \rightarrow \mathbb{A}, \mathfrak{s})$ is a Heegaard diagram for $\mathcal{T}$ and

$$
\left(\Sigma^{\circ}, \boldsymbol{\alpha} \backslash\{\alpha\}, \boldsymbol{\beta} \backslash\{\beta\}, \mathfrak{u}: \mathbf{z} \rightarrow \mathbb{A}, \mathfrak{s}_{1}\right)
$$

is a Heegaard diagram from $\mathcal{T}_{1}$, where $\mathfrak{s}_{1}=\left.\mathfrak{t}\right|_{M_{1}}$.

- There is a third collection $\gamma$ of pairwise disjoint circles on $\Sigma$ so that $\gamma \cap A$ is a single circle $\gamma$ which is a small Hamiltonian isotope of both $\alpha$ and $\beta$ and

$$
\left(\Sigma^{\circ}, \boldsymbol{\alpha} \backslash\{\alpha\}, \boldsymbol{\beta} \backslash\{\beta\}, \boldsymbol{\gamma} \backslash\{\gamma\}, \mathbf{z}\right)
$$

is subordinate to the framed $\operatorname{arc} \mathbb{I}$ in $\mathcal{T}_{1}$. In particular, the diagram $\left(\Sigma^{\circ}, \boldsymbol{\alpha} \backslash\{\alpha\}, \gamma \backslash\{\gamma\}, \mathbf{z}\right)$ is a Heegaard diagram for $\left(M^{\prime}, T^{\prime}\right)$.

Note that $(\Sigma, \boldsymbol{\alpha}, \boldsymbol{\beta}, \boldsymbol{\gamma}, \mathbf{z})$ is subordinate to $\mathbb{I}$, where $\mathbb{I}$ is considered as a framed arc in $\mathcal{T}$. Consider such a Heegaard diagram and assume that the almost complex structure is sufficiently stretched along the boundary circles of $A$. Every generator of $\mathrm{CF}^{\mathbb{M}}(\mathcal{T})$ is of the form $\mathbf{x} \times \theta$, where $\mathbf{x}$ is a generator of $\mathrm{CF}^{\mathrm{M}}\left(\mathcal{T}_{1}\right)$ and $\theta$ is one of the two intersection points $\theta_{\alpha \beta}$ and $\theta_{\beta \alpha}$ between $\alpha$ and $\beta$ which correspond to $\mathbb{S}$. The image of such a generator under $\mathfrak{f}_{\mathcal{C}_{1}}^{\mathbb{M}}$ is trivial unless $\theta=\theta_{\beta \alpha}$, when we have $\mathfrak{f}_{\mathcal{C}_{1}}^{\mathbb{M}}\left(\mathbf{x} \times \theta_{\beta \alpha}\right)=\mathbf{x}$. On the other hand, we may use the argument of Theorem 7.3 and show that

$$
\mathfrak{f}_{\mathcal{C}_{1}^{r}}^{\mathbb{M}}(\mathbf{x} \times \theta)=\mathfrak{f}_{\mathcal{C}_{2}}^{\mathbb{M}}(\mathbf{x}) \times \theta^{\prime}
$$

where $\theta^{\prime} \in \alpha \cap \gamma$ is the intersection point which corresponds to $\theta$. Every such generator is mapped to 0 by $\mathfrak{f}_{\mathcal{C}_{2}^{r}}^{\mathbb{M}}$ unless $\theta^{\prime}=\theta_{\gamma \alpha}$, or equivalently, unless $\theta=\theta_{\beta \alpha}$. If this condition is satisfied, then

$$
\mathfrak{f}_{\mathcal{C}_{2}^{r}}^{\mathbb{M}}\left(\mathfrak{f}_{\mathcal{C}_{1}^{r}}^{\mathbb{M}}\left(\mathbf{x} \times \theta_{\beta \alpha}\right)\right)=\mathfrak{f}_{\mathcal{C}_{2}}^{\mathbb{M}}(\mathbf{x})
$$

which completes the proof of the third case. The proof of the fifth case is similar to the proof of the third case.

The composition law of Theorem 7.5 implies, in particular, that the left-hand-side expression is well-defined. Let us define

$$
W^{0}=\bigcup_{i \equiv 0 \bmod 2} W_{i} \quad \text { and } \quad W^{1}=\bigcup_{i \equiv 1 \bmod 2} W_{i} .
$$

Then $W^{0} \cup W^{1}=W$ and $W^{0} \cap W^{1}=\cup_{i=1}^{m-1} M_{i}$, where $M_{i}=M_{\mathcal{T}_{i}}$, and we obtain the following cohomology long exact sequence

$$
\cdots \longrightarrow \bigoplus_{i=1}^{m-1} H^{1}\left(M_{i}, \mathbb{Z}\right) \stackrel{\delta}{\longrightarrow} H^{2}(W) \stackrel{\pi}{\longrightarrow} \bigoplus_{i=1}^{m} H^{2}\left(W_{i}, \mathbb{Z}\right) \longrightarrow \cdots
$$

If $\mathfrak{t}, \mathfrak{t}^{\prime} \in \operatorname{Spin}^{c}(W)$ restrict to $\mathfrak{t}_{i}$ on $W_{i}$, then $\mathfrak{t}-\mathfrak{t}^{\prime} \in H^{2}(M, \mathbb{Z})$ is a class in the kernel of $\pi$, and is thus in the image of $\delta$. In particular, the subset $\mathfrak{T} \subset \operatorname{Spin}^{c}(W)$ which appears in the summation of the left-hand-side of Equation 6 is the orbit of a fixed $\operatorname{Spin}^{c}$ structure $\mathfrak{t}$ under the action of the $\mathbb{Z}$-module $\operatorname{Im}(\delta)$ of $H^{2}(W, \mathbb{Z})$. On the other hand, using an appropriate Morse datum $\mathfrak{M}$, we may represent every $\mathbb{Z}$-submodule of $H^{2}(W, \mathbb{Z})$ as $\operatorname{Im}(\delta)$ for some decomposition of the stable cobordism $(W, F)$. In particular, for every affine set $\mathfrak{T}$ of $\operatorname{Spin}^{c}$ structures over a $\mathbb{Z}$-submodule of $H^{2}(W, \mathbb{Z})$ which restrict to $\mathfrak{s}$ and $\mathfrak{s}^{\prime}$ on the two ends, the sum

$$
\sum_{\mathfrak{t} \in \mathfrak{T}} \mathfrak{f}_{\mathcal{C}_{\mathfrak{t}}}^{\mathbb{M}}: \operatorname{HF}^{\mathbb{M}}(\mathcal{T}) \rightarrow \operatorname{HF}^{\mathbb{M}}\left(\mathcal{T}^{\prime}\right)
$$


is well-defined.

Definition 7.1. Let $\mathcal{C}=\left[W, F, \mathfrak{T}, \mathfrak{u}_{F}\right]: \mathcal{T} \leadsto \mathcal{T}^{\prime}$ be an arbitrary $\mathbb{A}$-cobordism from the $\mathbb{A}$-tangle $\mathcal{T}$ to the $\mathbb{A}$-tangle $\mathcal{T}^{\prime}$, where $\mathfrak{T}$ is a subset of $\operatorname{Spin}^{c}(W)$ which is affine over a $\mathbb{Z}$-submodule of $H^{2}(W, \mathbb{Z})$. For every $\mathfrak{t} \in \mathfrak{T}$ define $\mathcal{C}_{\mathfrak{t}}=[W, F, \mathfrak{t}, \mathfrak{u}]$. We then define the cobordism map associated with $\mathcal{C}$ by

$$
\mathfrak{f}_{\mathcal{C}}^{\mathbb{M}}:=\sum_{\mathfrak{t} \in \mathfrak{T}} \mathfrak{f}_{\mathcal{C}_{\mathfrak{t}}}^{\mathbb{M}}
$$

With the above definition in place, we may then re-state Theorem 7.1 and Theorem 7.3 as the following theorem.

Theorem 7.6. Fix an algebra $\mathbb{A}$ over $\mathbb{F}$ and an $\mathbb{A}$-module $\mathbb{M}$. Assigning the $\mathbb{A}$-module $\operatorname{HF}^{\mathbb{M}}(\mathcal{T})$ to every $\mathbb{A}$-tangle $\mathcal{T} \in \operatorname{Obj}\left(\mathbb{A}\right.$-Tangles) and the $\mathbb{A}$-homomorphism $\mathfrak{f}_{\mathcal{C}}^{\mathbb{M}}: \operatorname{HF}^{\mathbb{M}}(\mathcal{T}) \rightarrow \operatorname{HF}^{\mathbb{M}}\left(\mathcal{T}^{\prime}\right)$ to every A-cobordism

$$
\left(\mathcal{C}: \mathcal{T} \leadsto \mathcal{T}^{\prime}\right) \in \operatorname{Mor}\left(\mathcal{T}, \mathcal{T}^{\prime}\right) \in \operatorname{Mor}(\mathbb{A} \text {-Tangles })
$$

gives a well-defined functor

$$
\mathrm{HF}^{\mathbb{M}}: \mathbb{A} \text {-Tangles } \longrightarrow \mathbb{A} \text {-Modules. }
$$

7.4. Action of $\Lambda^{*}\left(H_{1}(W, \mathbb{Z}) /\right.$ Tors $)$. Let us assume that $\mathcal{C}=\left[W, F, \mathfrak{t}, \mathfrak{u}_{F}\right]$ is an $\mathbb{A}$-cobordism from $\mathcal{T}=[M, T, \mathfrak{s}, \mathfrak{u}]$ to $\mathcal{T}^{\prime}=\left[M^{\prime}, T^{\prime}, \mathfrak{s}^{\prime}, \mathfrak{u}^{\prime}\right]$. Consider a decomposition of $\mathcal{C}$ as

$$
\mathcal{C}=\mathcal{C}_{1} \cup_{\mathcal{T}_{1}} \mathcal{C}_{2} \cup_{\mathcal{T}_{2}} \mathcal{C}_{3}
$$

where $\mathcal{C}_{1}$ corresponds to the addition of 1 -handles, $\mathcal{C}_{2}$ corresponds to the addition of 2 -handles along some framed link and band surgeries along framed arcs, and $\mathcal{C}_{3}$ corresponds to the addition of 3-handles. Let $\left(M_{i}, T_{i}\right)$ be the underlying tangle of $\mathcal{T}_{i}$ and $\mathcal{C}^{\prime}=\mathcal{C}_{2}=\left[W^{\prime}, F^{\prime}, \mathfrak{t}^{\prime}, \mathfrak{u}_{F^{\prime}}\right]$. It is clear that $H_{1}\left(W^{\prime}, \mathbb{Z}\right)=H_{1}(W, \mathbb{Z})$.

Assume $\mathcal{C}^{\prime}$ is parametrized by a pair $(\mathbb{I}, \mathbb{S})$ of an acceptable set of framed arcs $\mathbb{I}$, and a framed link $\mathbb{S}$ such that $M_{2}=M_{1}(\mathbb{S})$ and $T_{2}=T_{1}(\mathbb{I})$. The homomorphism $\mathfrak{f}_{\mathcal{C}^{\prime}}^{\mathbb{M}}=\mathfrak{f}_{\mathcal{C}^{\prime}, \mathbb{I}, \mathbb{S}}^{\mathbb{M}}$ is defined using an A-diagram

$$
\mathcal{H}=(\Sigma, \boldsymbol{\alpha}, \boldsymbol{\beta}, \boldsymbol{\gamma}, \mathfrak{u}, \mathfrak{t})
$$

subordinate to $(\mathbb{I}, \mathbb{S})$. Let us denote by $W_{\alpha \beta \gamma}$ the 4 -manifold obtained from the Heegaard triple $\mathcal{H}$. There is an epimorphism

$$
\pi: H_{1}\left(M_{\alpha \beta} \amalg M_{\beta \gamma} \amalg M_{\alpha \gamma}, \mathbb{Z}\right) / \text { Tors } \longrightarrow H_{1}\left(W_{\alpha \beta \gamma}, \mathbb{Z}\right) / \text { Tors }=H_{1}(W, \mathbb{Z}) / \text { Tors. }
$$

Every element $\zeta \in H_{1}(W, \mathbb{Z}) /$ Tors may be represented as $\pi\left(\zeta_{\alpha \beta}, \zeta_{\beta \gamma}, \zeta_{\alpha \gamma}\right)$ with

$$
\left(\zeta_{\alpha \beta}, \zeta_{\beta \gamma}, \zeta_{\alpha \gamma}\right) \in H_{1}\left(M_{\alpha \beta} \amalg M_{\beta \gamma} \amalg M_{\alpha \gamma}, \mathbb{Z}\right) / \text { Tors. }
$$

We may then define

$$
\begin{aligned}
& \mathfrak{f}_{\mathcal{H}}^{\zeta}: \operatorname{HF}\left(\Sigma, \boldsymbol{\alpha}, \boldsymbol{\beta}, \mathfrak{u},\left.\mathfrak{t}\right|_{M_{\alpha \beta}}\right) \longrightarrow \operatorname{HF}\left(\Sigma, \boldsymbol{\alpha}, \gamma, \mathfrak{u},\left.\mathfrak{t}\right|_{M_{\alpha \gamma}}\right) \\
& \mathfrak{f}_{\mathcal{H}}^{\zeta}(\mathbf{x}):=\mathfrak{f}_{\alpha \beta \gamma}\left(\left(\zeta_{\alpha \beta} \cdot \mathbf{x}\right) \otimes \Theta_{\beta \gamma}+\mathbf{x} \otimes\left(\zeta_{\beta \gamma} \cdot \Theta_{\beta \gamma}\right)\right)-\zeta_{\alpha \gamma} \cdot \mathfrak{f}_{\alpha \beta \gamma}\left(\mathbf{x} \otimes \Theta_{\beta \gamma}\right) .
\end{aligned}
$$

Correspondingly, we may define

$$
\overline{\mathfrak{f}}_{\mathcal{H}}^{\mathbb{M}}: \operatorname{HF}^{\mathbb{M}}\left(\mathcal{T}_{1}\right) \otimes \Lambda^{*}\left(H_{1}(W, \mathbb{Z}) / \text { Tors }\right) \longrightarrow \operatorname{HF}^{\mathbb{M}}\left(\mathcal{T}_{2}\right), \quad \overline{\mathfrak{f}}_{\mathcal{H}}^{\mathbb{M}}(\mathbf{x} \otimes \zeta):=\mathfrak{f}_{\mathcal{H}}^{\zeta}(\mathbf{x}) .
$$

It is then implied by [OS06, Lemma 2.6] that the above map is in fact well-defined, and does not depend on the representation of $\zeta$ as $\pi\left(\zeta_{\alpha \beta}, \zeta_{\beta \gamma}, \zeta_{\alpha \gamma}\right)$. After composing with the maps $\mathfrak{f}_{\mathcal{C}_{1}}^{\mathbb{M}}$ and $\mathfrak{f}_{\mathcal{C}_{3}}^{\mathbb{M}}$ we obtain an induced map, which may be denoted by

$$
\overline{\mathfrak{f}}_{\mathcal{C}}^{\mathbb{M}}: \operatorname{HF}^{\mathbb{M}}(\mathcal{T}) \otimes \Lambda^{*}\left(H_{1}(W, \mathbb{Z}) / \text { Tors }\right) \longrightarrow \operatorname{HF}^{\mathbb{M}}\left(\mathcal{T}^{\prime}\right)
$$

We may then follow the steps taken in OS06 to show the invariance of the map $\mathfrak{f}_{\mathcal{C}}^{\mathbb{M}}$ and show that $\overline{\mathfrak{f}}_{\mathcal{C}}^{\mathbb{M}}$ is also well-defined. 
7.5. Relative $\operatorname{Spin}^{c}$ structures and the cobordism map. Suppose $\mathcal{W}=(W, F)$ is a stable cobordism from a balanced tangle $\left(M^{0}, T^{0}\right)$ to a balanced tangle $\left(M^{1}, T^{1}\right)$. Let $\left(X^{i}, \tau^{i}\right)$ be the sutured manifold associated with $\left(M^{i}, T^{i}\right)$ for $i=0,1$. Specifically, $X^{i}=M^{i} \backslash \operatorname{nd}\left(T^{i}\right)$ for a tubular neighborhood $\operatorname{nd}\left(T^{i}\right)$ around $T^{i}$. Moreover, if $T^{i}=\amalg_{j=1}^{\kappa} T_{j}^{i}$, then $\tau^{i}=\amalg_{j=1}^{\kappa} \tau_{j}^{i}$ where $\tau_{j}^{i} \subset \partial \operatorname{nd}\left(T^{i}\right)$ is the meridian of $T_{j}^{i}$ for each $1 \leq j \leq \kappa$.

Denote the Poincaré dual of the homology class $\left[\tau_{j}^{i}\right] \in H_{1}\left(X^{i}, \mathbb{Z}\right)$ by $\chi_{j}^{i} \in \mathrm{H}^{2}\left(X^{i}, \partial X^{i}, \mathbb{Z}\right)$. Recall that the set of relative $\operatorname{Spin}^{c}$ classes on $\left(M^{i}, T^{i}\right)$, denoted by $\operatorname{Spin}^{c}\left(M^{i}, T^{i}\right)$, is an affine space over $\mathrm{H}^{2}\left(X^{i}, \partial X^{i}, \mathbb{Z}\right)$ and sits in the exact sequence

$$
0 \longrightarrow\left\langle\chi_{j}^{i} \mid 1 \leq j \leq \kappa\right\rangle_{\mathbb{Z}} \longrightarrow \operatorname{Spin}^{c}\left(M^{i}, T^{i}\right) \longrightarrow \operatorname{Spin}^{c}\left(M^{i}\right) \longrightarrow 0 .
$$

The surface $F$ induces an equivalence relation on the components of $T^{i}$ by setting $T_{j}^{i} \sim T_{k}^{i}$ if they are subsets of the boundary of the same component of $F$. Let

$$
\mathcal{I}_{i}=\left\langle\chi_{j}^{i}-\chi_{k}^{i} \mid T_{j}^{i} \sim T_{k}^{i}\right\rangle \subset \mathrm{H}^{2}\left(X^{i}, \partial X^{i}, \mathbb{Z}\right)
$$

and define

$$
\operatorname{Spin}_{F}^{c}\left(M^{i}, T^{i}\right):=\frac{\operatorname{Spin}^{c}\left(M^{i}, T^{i}\right)}{\mathcal{I}_{i}} \quad \text { and } \quad \mathbb{H}_{F}^{i}:=\frac{\mathrm{H}^{2}\left(X^{i}, \partial X^{i}, \mathbb{Z}\right)}{\mathcal{I}_{i}}
$$

Consequently, if $F=\amalg_{j=1}^{m} F_{j}$, each component $F_{j}$ of $F$ determines a equivalence class in $\mathbb{H}_{F}^{i}$, denoted by $\eta_{j}^{i}$.

Correspondingly, this equivalence relation specifies an $\mathbb{F}$-algebra $(\mathbb{F}=\mathbb{Z} / 2 \mathbb{Z})$, denoted by $\mathbb{A}_{F}$, which is isomorphic to a quotient of $\mathbb{A}_{i}=\mathbb{A}_{\left(M_{i}, T_{i}\right)} \otimes_{\mathbb{Z}} \mathbb{F}$ by the ideal determined by $\sim$. More precisely, consider the polynomial ring $\mathbb{F}\left[\mathfrak{u}_{1}, \ldots, \mathfrak{u}_{m}\right]$. If $\partial^{+} M^{0}=\amalg_{i=1}^{k} S_{i}^{-}$and $\partial^{-} M^{0}=\amalg_{j=1}^{l} S_{j}^{+}$, then

$$
\partial_{h}^{-} W=\amalg_{i=1}^{k} S_{i}^{-} \times[0,1] \quad \text { and } \quad \partial_{h}^{+} W=\amalg_{j=1}^{l} S_{j}^{+} \times[0,1] .
$$

Associated with any connected component of $\partial_{h} W$, we define

$$
\mathfrak{u}_{i}^{-}:=\prod_{F_{j} \cap S_{i}^{-} \neq \emptyset} \mathfrak{u}_{j} \quad \text { for } 1 \leq i \leq k, \mathfrak{u}_{i}^{+}:=\prod_{F_{j} \cap S_{i}^{+} \neq \emptyset} \mathfrak{u}_{j} \quad \text { for } \quad 1 \leq i \leq l .
$$

If we set $\mathfrak{u}^{-}=\sum_{i=1}^{k} \mathfrak{u}_{i}^{-}$and $\mathfrak{u}^{+}=\sum_{i=1}^{l} \mathfrak{u}_{i}^{+}$, it follows that

$$
\mathbb{A}_{F}:=\frac{\mathbb{F}\left[\mathfrak{u}_{1}, \ldots, \mathfrak{u}_{m}\right]}{\left\langle\mathfrak{u}_{i}^{-} \mid g\left(S_{i}^{-}\right)>0\right\rangle+\left\langle\mathfrak{u}_{i}^{+} \mid g\left(S_{i}^{+}\right)>0\right\rangle+\left\langle\mathfrak{u}^{+}-\mathfrak{u}^{-}\right\rangle} .
$$

The map $\mathfrak{u}_{F}: \pi_{0}(F) \rightarrow \mathbb{A}_{F}$ mapping $F_{i}$ to $\mathfrak{u}_{i}$ for $i=1, \ldots, m$, is an $\mathbb{A}_{F}$-coloring on $(W, F)$. Thus, $\mathcal{C}=\left[W, F, \mathfrak{t}, \mathfrak{u}_{F}\right]$ is an $\mathbb{A}_{F}$-cobordism for every $\mathfrak{t} \in \operatorname{Spin}^{c}(W)$. Let $\mathfrak{u}^{i}: \pi_{0}\left(T^{i}\right) \rightarrow \mathbb{A}_{F}$ denote the $\mathbb{A}_{F}$-coloring induced by $\mathfrak{u}_{F}$ on $\left(M_{i}, T_{i}\right)$. Associated with this coloring, there is a natural filtration on $\mathbb{A}_{F}$ by $\mathbb{H}_{F}^{i}$ defined by

$$
\chi^{i}: G\left(\mathbb{A}_{F}\right) \rightarrow \mathbb{H}_{F}^{i}=\mathrm{H}^{2}\left(X^{i}, \partial X^{i}, \mathbb{Z}\right) \quad \chi^{i}\left(\prod_{j=1}^{m} \mathfrak{u}_{j}^{a_{j}}\right):=\sum_{j=1}^{m} a_{j} \eta_{j}^{i},
$$

see [AE15, Section 3.2]. Thus, for any $\operatorname{Spin}^{c} \operatorname{class} \mathfrak{s}^{i} \in \operatorname{Spin}^{c}\left(M^{i}\right)$ we have

$$
\operatorname{HF}\left(M^{i}, T^{i}, \mathfrak{s}^{i}, \mathfrak{u}^{i}\right)=\bigoplus_{\underline{\mathfrak{s}}^{i} \in \mathfrak{s}^{i} \subset \operatorname{Spin}_{F}^{c}\left(M^{i}, T^{i}\right)} \operatorname{HF}\left(M^{i}, T^{i}, \underline{\mathfrak{s}}^{i}, \mathfrak{u}^{i}\right)
$$

Lemma 7.7. Consider the $\mathbb{A}_{F}$-cobordism $\mathcal{C}=\left[W, F, \mathfrak{t}, \mathfrak{u}_{F}\right]$ as above and let $\mathfrak{s}^{0}=\left.\mathfrak{t}\right|_{M^{0}}$ and $\mathfrak{s}^{1}=\left.\mathfrak{t}\right|_{M^{1}}$. Suppose that for an element $\mathbf{x}$ in $\operatorname{HF}\left(M^{0}, T^{0}, \underline{\mathfrak{s}}^{0}, \mathfrak{u}^{0}\right)$ with $\underline{\mathfrak{s}}^{0} \in \mathfrak{s}^{0}$ we have $\mathfrak{f}_{\mathcal{C}}(\mathbf{x}) \in \operatorname{HF}\left(M^{1}, T^{1}, \underline{\mathfrak{s}}^{1}, \mathfrak{u}^{1}\right)$ where $\underline{\mathfrak{s}}^{1} \in \mathfrak{s}^{1}$. Then, $\mathfrak{f}_{\mathcal{C}}$ induces the maps

$$
\mathfrak{f}_{\mathcal{C}}: \operatorname{HF}\left(M^{0}, T^{0}, \underline{\mathfrak{s}}^{0}+\sum_{j=1}^{m} a_{j} \eta_{j}^{0}, \mathfrak{u}^{0}\right) \rightarrow \operatorname{HF}\left(M^{1}, T^{1}, \underline{\mathfrak{s}}^{1}+\sum_{j=1}^{m} a_{j} \eta_{j}^{1}, \mathfrak{u}^{1}\right),
$$

for every choice of $a_{1}, \ldots, a_{m} \in \mathbb{Z}$. 
Proof. It is enough to prove the lemma in the case where $(W, F)$ is determined by $(\mathbb{I}, \mathbb{S})$, for an acceptable set of framed arcs $\mathbb{I}$ and a framed link $\mathbb{S}$. Let

$$
\mathcal{H}=\left(\Sigma, \boldsymbol{\alpha}, \boldsymbol{\beta}, \boldsymbol{\gamma}, \mathfrak{t}, \mathfrak{u}_{F}\right)
$$

be an $\mathbb{A}_{F}$-diagram for $\mathcal{C}$ whose underlying Heegaard triple is subordinate to $(\mathbb{I}, \mathbb{S})$. Further, assume that the distinguished generator $\Theta_{\beta \gamma}$ is represented by an intersection point $\theta_{\beta \gamma} \in \mathbb{T}_{\beta} \cap \mathbb{T}_{\gamma}$ and the marked points in $\mathbf{z}$ are labelled such that $z_{i}$ corresponds to $T_{i}^{0}$. Consider the intersection points $\mathbf{x}, \mathbf{y} \in \mathbb{T}_{\alpha} \cap \mathbb{T}_{\beta}$ and let

$$
\mathfrak{\mathfrak { s }}(\mathbf{y})-\underline{\mathfrak{s}}(\mathbf{x})=\sum_{j=1}^{m} a_{j} \eta_{j}^{0},
$$

where $a_{j} \in \mathbb{Z}$ for all $j$. Here, abusing the notation $\underline{\mathfrak{s}}(\cdot)$ denotes the equivalence class of the relative $\operatorname{Spin}^{c}$ structure represented by the corresponding generator. Let $\Delta_{x} \in \pi_{2}\left(\mathbf{x}, \Theta_{\beta \gamma}, \mathbf{x}^{\prime}\right)$ and $\Delta_{y} \in \pi_{2}\left(\mathbf{y}, \Theta_{\beta \gamma}, \mathbf{y}^{\prime}\right)$ be triangle classes representing the $\operatorname{Spin}^{c}$ structure $\mathfrak{t}$ for some $\mathbf{x}^{\prime}, \mathbf{y}^{\prime} \in \mathbb{T}_{\alpha} \cap \mathbb{T}_{\gamma}$. Then, there are disks $\phi \in \pi_{2}(\mathbf{y}, \mathbf{x})$ and $\psi \in \pi_{2}\left(\mathbf{x}^{\prime}, \mathbf{y}^{\prime}\right)$ such that $\Delta_{y}=\phi \star \Delta_{x} \star \psi$. Since $a_{j}=n_{j}(\phi)=$ $\sum_{T_{i}^{0} \subset \partial F_{j}} n_{z_{i}}(\phi)$,

$$
\begin{aligned}
\underline{\mathfrak{s}}\left(\prod_{j=1}^{m} \mathfrak{u}_{j}^{n_{j}\left(\Delta_{y}\right)} \cdot \mathbf{y}^{\prime}\right)-\underline{\mathfrak{s}}\left(\prod_{j=1}^{m} \mathfrak{u}_{j}^{n_{j}\left(\Delta_{x}\right)} \cdot \mathbf{x}^{\prime}\right) & =\sum_{j=1}^{m}\left(n_{j}\left(\Delta_{y}\right)-n_{j}\left(\Delta_{x}\right)\right) \eta_{j}^{1}+\underline{\mathfrak{s}}\left(\mathbf{y}^{\prime}\right)-\underline{\mathfrak{s}}\left(\mathbf{x}^{\prime}\right) \\
& =\sum_{j=1}^{m}\left(n_{j}\left(\Delta_{y}\right)-n_{j}\left(\Delta_{x}\right)\right) \eta_{j}^{1}-\sum_{j=1}^{m} n_{j}(\psi) \eta_{j}^{1} \\
& =\sum_{j=1}^{m} n_{j}(\phi) \eta_{j}^{1}=\sum_{j=1}^{m} a_{j} \eta_{j}^{1}
\end{aligned}
$$

where $n_{j}\left(\Delta_{\bullet}\right)=\sum_{T_{i}^{0} \subset \partial F_{j}} n_{z_{i}}\left(\Delta_{\bullet}\right)$ for $\bullet=x, y$ and $n_{j}(\psi)=\sum_{T_{i}^{0} \subset \partial F_{j}} n_{z_{i}}(\psi)$. This completes the proof of lemma. 


\section{Applichtions And special CASES}

8.1. Cobordisms between closed 3-manifolds. Let $\mathcal{Y}=(Y, p)$ be an oriented, closed 3-manifold $Y$ with a based point $p \in Y$. Associated with $\mathcal{Y}$, there is a balanced tangle $\left(Y_{p}, T_{p}\right)$ defined as follows. Let $p_{-}, p_{+} \in Y$ be points close to $p$ and $T \subset Y$ be an embedded oriented arc passing through $p$ such that $\partial^{-} T=p_{-}$and $\partial^{+} T=p_{+}$. Then, $Y_{p}$ is constructed by removing small disjoint balls (also disjoint from $p$ ) around $p_{-}$and $p_{+}$and $T_{p}=T \cap Y_{p}$. Note that $\partial^{-} Y_{p}$ and $\partial^{+} Y_{p}$ are the boundary of spheres around $p_{-}$and $p_{+}$, respectively, and $T_{p}$ has one connected component. So, there is an obvious $\mathbb{F}[\mathfrak{u}]$-coloring on $\left(Y_{p}, T_{p}\right)$ labeling $T_{p}$ by $\mathfrak{u}$. Here, as before $\mathbb{F}=\mathbb{Z} / 2 \mathbb{Z}$. Let $\mathcal{T}_{\mathcal{Y}, \mathfrak{s}}=\left(Y_{p}, T_{p}, \mathfrak{s}, \mathfrak{u}_{p}\right)$ for every $\mathfrak{s} \in \operatorname{Spin}^{c}(Y)$.

Definition 8.1. Let $\mathcal{Y}=(Y, p)$ and $\mathcal{Y}^{\prime}=\left(Y^{\prime}, p^{\prime}\right)$ be oriented, closed, based 3-manifolds. A decorated cobordism $\mathcal{X}=(X, \sigma)$ from $\mathcal{Y}$ to $\mathcal{Y}^{\prime}$ is a smooth, oriented 4-manifold $X$ with $\partial X=$ $-Y \amalg Y^{\prime}$ and a properly embedded $\operatorname{arc} \sigma \subset X$ such that $\partial \sigma=p \amalg p^{\prime}$.

Associated with any decorated cobordism $\mathcal{X}=(X, \sigma)$ from $\mathcal{Y}=(Y, p)$ to $\mathcal{Y}^{\prime}=\left(Y^{\prime}, p^{\prime}\right)$ one may construct a stable cobordism $\left(X_{\sigma}, F_{\sigma}\right)$ from $\left(Y_{p}, T_{p}\right)$ to $\left(Y_{p}^{\prime}, T_{p}^{\prime}\right)$, as follows. Let $T \subset Y$ and $T^{\prime} \subset Y^{\prime}$ be embedded, oriented arcs containing $p$ and $p^{\prime}$ respectively, and let $\partial^{\bullet} T=p_{\bullet}$ and $\partial^{\bullet} T^{\prime}=p_{\bullet}^{\prime}$ for $\bullet=+,-$. Consider parallel disjoint copies of $\sigma$ in $X$, denoted by $\sigma^{-}$and $\sigma^{+}$, such that $\partial \sigma^{-}=p_{-} \amalg p_{-}^{\prime}, \partial \sigma^{+}=p_{+} \amalg p_{+}^{\prime}$ and $\sigma^{-} \cup T^{\prime} \cup \sigma^{+} \cup T$ bounds an embedded disk $D$ in $X$. Then, $X_{\sigma}$ is obtained from $X$ by removing small, disjoint tubular neighborhoods around $\sigma^{-}$and $\sigma^{+}$, while $F_{\sigma}=D \cap X_{\sigma}$. Let $\mathfrak{u}_{\sigma}$ denote the $\mathbb{F}[\mathfrak{u}]$-coloring on $\left(X_{\sigma}, F_{\sigma}\right)$ which labels $F_{\sigma}$ by $\mathfrak{u}$, and set $\mathcal{C}_{\mathcal{X}, \mathfrak{t}}=\left(X_{\sigma}, F_{\sigma}, \mathfrak{t}, \mathfrak{u}_{\sigma}\right)$ for every $\mathfrak{t} \in \operatorname{Spin}^{c}(X)$.

For every closed, oriented, based 3 -manifold $\mathcal{Y}=(Y, p)$ and every $\mathfrak{s} \in \operatorname{Spin}^{c}(Y)$, the homology groups $\operatorname{HF}^{\mathbb{M}}\left(\mathcal{T}_{\mathcal{Y}, \mathfrak{s}}\right)$, for $\mathbb{M}$ equal to $\mathbb{F}, \mathbb{F}[\mathfrak{u}], \mathbb{F}\left[\frac{1}{\mathfrak{u}}\right]$ or $\mathbb{F}\left[\mathfrak{u}, \frac{1}{\mathfrak{u}}\right]$, are equal to $\widehat{\operatorname{HF}}(Y, \mathfrak{s} ; \mathbb{F}), \operatorname{HF}^{-}(Y, \mathfrak{s} ; \mathbb{F})$, $\operatorname{HF}^{+}(Y, \mathfrak{s} ; \mathbb{F})$ and $\operatorname{HF}^{\infty}(Y, \mathfrak{s} ; \mathbb{F})$, respectively. Moreover, for every decorated cobordism $\mathcal{X}=(X, \sigma)$ from $(Y, p)$ to $\left(Y^{\prime}, p^{\prime}\right)$ and every $\operatorname{Spin}^{c}$ structure $\mathfrak{t} \in \operatorname{Spin}^{c}(X)$, the cobordism map $\mathfrak{f}_{\mathcal{C}_{\mathcal{X}, \mathfrak{t}}}^{\mathbb{M}}$ is the cobordism map of Ozsváth and Szabó in any of the aforementioned cases.

8.2. Functoriality of link Floer homology. Another important example of $\mathbb{A}$-tangles is given by multi-pointed links.

Definition 8.2. A multi-pointed link is a triple $\mathcal{L}=(Y, L, \mathbf{p})$ where $L$ is an oriented link in a closed, connected, oriented 3-manifold $Y$ together with a finite set $\mathbf{p} \subset L$ of based points such that every component of $L$ contains at least one base point.

Associated with any multi-pointed link $\mathcal{L}=(Y, L, \mathbf{p})$ we define a balanced tangle $\left(Y_{\mathbf{p}}, L_{\mathbf{p}}\right)$ as follows. Assume $\mathbf{p}=\left\{p_{1}, \ldots, p_{n}\right\}$ and consider $n$ pairwise of disjoint arc segments $I=\amalg_{i=1}^{n} I_{i}$ in $L$ such that $p_{i} \in I_{i}$. Using the orientation induced from $L$ on $I$, let $\mathbf{p}_{-}=\partial^{-} I$ and $\mathbf{p}_{+}=\partial^{+} I$. Then, $Y_{\mathbf{p}}$ is obtained from $Y$ by removing small disjoint ball neighborhoods around the points in $\mathbf{p}_{-}$and $\mathbf{p}_{+}$and $\partial \bullet Y_{\mathbf{p}} \subset \partial Y_{\mathbf{p}}$ is the union of sphere boundary components around $\mathbf{p} \bullet$ for $\bullet=+,-$. Furthermore,

$$
L_{\mathbf{p}}=-\left((L \backslash I) \cap Y_{\mathbf{p}}\right) \amalg\left(I \cap Y_{\mathbf{p}}\right) .
$$

Definition 8.3. A decorated cobordism from $\mathcal{L}=(Y, L, \mathbf{p})$ to $\mathcal{L}^{\prime}=\left(Y^{\prime}, L^{\prime}, \mathbf{p}^{\prime}\right)$ is a triple $\mathcal{F}=$ $(X, F, \sigma)$ as follows.

(1) $X$ is a smooth, oriented 4-manifold with $\partial X=-Y \amalg Y^{\prime}$.

(2) $F \subset X$ is a smoothly embedded, oriented surface such that $\partial F=-L \amalg L^{\prime}$.

(3) $\sigma \subset F$ is a union of embedded, pairwise disjoint, oriented arcs such that $\partial^{-} \sigma=\mathbf{p}, \partial^{+} \sigma=\mathbf{p}^{\prime}$ and every component of $F \backslash \sigma$ with positive genus intersects more than one component of $L \backslash \mathbf{p}$ and $L^{\prime} \backslash \mathbf{p}^{\prime}$.

To any decorated cobordism $\mathcal{F}=(X, F, \sigma)$ from $\mathcal{L}=(Y, L, \mathbf{p})$ to $\mathcal{L}^{\prime}=\left(Y^{\prime}, L^{\prime}, \mathbf{p}^{\prime}\right)$, we assign a cobordism $\left(X_{\sigma}, F_{\sigma}\right)$ from the tangle $\left(Y_{\mathbf{p}}, L_{\mathbf{p}}\right)$ to $\left(Y_{\mathbf{p}^{\prime}}^{\prime}, L_{\mathbf{p}^{\prime}}^{\prime}\right)$. We choose the labeling for $\mathbf{p}=$ $\amalg_{i=1}^{n} p_{i}, \mathbf{p}^{\prime}=\amalg_{i=1}^{n} p_{i}^{\prime}$ and $\sigma=\amalg_{i=1}^{n} \sigma_{i}$ such that $\partial \sigma_{i}=p_{i} \amalg p_{i}^{\prime}$ for all $1 \leq i \leq n$. Let $I=\amalg_{i=1}^{n} I_{i}$ and 
$I^{\prime}=\amalg_{i=1}^{n} I_{i}^{\prime}$ where $I_{i} \subset L$ and $I_{i}^{\prime} \subset L^{\prime}$ are connected segments containing $p_{i}$ and $p_{i}^{\prime}$, respectively. Let

$$
\mathbf{p}_{\bullet}=\partial^{\bullet} I=\amalg_{i=1}^{n} p_{i \bullet} \quad \text { and } \quad \mathbf{p}_{\bullet}^{\prime}=\partial^{\bullet} I^{\prime}=\amalg_{i=1}^{n} p_{i \bullet}^{\prime}
$$

where $p_{i \bullet}=\partial^{\bullet} I_{i}$ and $p_{i \bullet}^{\prime}=\partial^{\bullet} I_{i}^{\prime}$ for $\bullet=-,+$. Consider parallel copies $\sigma_{i}^{-}, \sigma_{i}^{+} \subset F$ of each $\sigma_{i}$ such that $\partial \sigma_{i}^{\bullet}=p_{i \bullet} \amalg p_{i \bullet}^{\prime}$ for $\bullet=-,+$. Moreover, $\sigma_{i}^{-} \cup \sigma_{i}^{+} \cup I_{i} \cup I_{i}^{\prime}$ bounds a disk $D_{i} \subset F$ containing $\sigma_{i}$ such that $D_{1}, \ldots, D_{n}$ are pairwise disjoint. Let $F^{\circ}=F \backslash\left(\amalg_{i=1}^{n} D_{i}\right)=\amalg_{j=1}^{m} F_{j}^{\circ}$. For each $1 \leq i \leq n$, $m \bullet(i)$ is defined such that $\sigma_{i}^{\bullet} \subset \partial F_{m}^{\circ} \bullet(i)$ where $\bullet=+,-$.

Then, $X_{\sigma}$ is constructed from $X$ by removing disjoint small tubular neighborhood of the arcs $\amalg_{i=1}^{n}\left(\sigma_{i}^{-} \amalg \sigma_{i}^{+}\right)$while

$$
F_{\sigma}=-\left(F^{\circ} \cap X_{\sigma}\right) \amalg\left(\amalg_{i=1}^{n} D_{i} \cap X_{\sigma}\right) .
$$

Abusing the notation, we denote $D_{i} \cap X_{\sigma}$ by $D_{i}$ and $F_{j}^{\circ} \cap X_{\sigma}$ by $F_{j}^{\circ}$ where $F^{\circ}=\amalg_{j=1}^{m} F_{j}^{\circ}$. The algebra associated to the cobordism $\left(X_{\sigma}, F_{\sigma}\right)$, defined as in Equation 8, is equal to

$$
\mathbb{A}=\frac{\mathbb{F}\left[\mathfrak{v}_{1}, \ldots, \mathfrak{v}_{m}, \mathfrak{u}_{1}, \ldots, \mathfrak{u}_{n}\right]}{\sum_{i=1}^{n} \mathfrak{u}_{i} \mathfrak{v}_{m^{+}(i)}-\sum_{i=1}^{n} \mathfrak{u}_{i} \mathfrak{v}_{m^{-}(i)}},
$$

and the map $\mathfrak{u}_{\sigma}: \pi_{0}\left(F_{\sigma}\right) \rightarrow \mathbb{A}$, defined by

$$
\begin{cases}\mathfrak{u}\left(F_{j}^{\circ}\right)=\mathfrak{v}_{j} & 1 \leq j \leq m \\ \mathfrak{u}\left(D_{i}\right)=\mathfrak{u}_{i} & 1 \leq i \leq n,\end{cases}
$$

is an $\mathbb{A}$-coloring on $\left(X_{\sigma}, F_{\sigma}\right)$. So, $\mathcal{C}_{\mathcal{F}, \mathfrak{t}}=\left[W_{\sigma}, F_{\sigma}, \mathfrak{t}, \mathfrak{u}_{\sigma}\right]$ is an $\mathbb{A}$-cobordism for every $\mathfrak{t} \in \operatorname{Spin}^{c}(X)$. Let $\mathcal{T}_{\mathcal{L}, \mathfrak{s}}$ (respectively $\mathcal{T}_{\mathcal{L}^{\prime}, \mathfrak{s}^{\prime}}$ ) denote the $\mathbb{A}$-tangle obtained from equipping $\left(Y_{\mathbf{p}}, L_{\mathbf{p}}\right)$ (respectively $\left.\left(Y_{\mathbf{p}^{\prime}}^{\prime}, L_{\mathbf{p}^{\prime}}^{\prime}\right)\right)$ with the coloring induced from $\mathfrak{u}_{\sigma}$ and the $\operatorname{Spin}^{c}$ structure $\mathfrak{s}=\left.\mathfrak{t}\right|_{Y}\left(\right.$ respectively $\left.\mathfrak{s}^{\prime}=\left.\mathfrak{t}\right|_{Y^{\prime}}\right)$.

Then for every $\mathbb{A}$-module $\mathbb{M}$ we have an $\mathbb{A}$-homomorphism

$$
\mathfrak{f}_{\mathcal{C}_{\mathcal{F}, \mathfrak{t}}}^{\mathbb{M}}: \operatorname{HF}^{\mathbb{M}}\left(\mathcal{T}_{\mathcal{L}, \mathfrak{s}}\right) \rightarrow \operatorname{HF}^{\mathbb{M}}\left(\mathcal{T}_{\mathcal{L}^{\prime}, \mathfrak{s}^{\prime}}\right)
$$

For $\mathbb{M}=\mathbb{A}$, following the discussions of Section 7.5, the chain complexes $\operatorname{CF}\left(\mathcal{T}_{\mathcal{L}, \mathfrak{s}}\right)$ and $\operatorname{CF}\left(\mathcal{T}_{\mathcal{L}^{\prime}, \mathfrak{s}^{\prime}}\right)$ are $\left(\mathbb{A}, \mathbb{H}_{F}\right)$ and $\left(\mathbb{A}, \mathbb{H}_{F}^{\prime}\right)$ filtered chain complexes where $\mathbb{H}_{F}$ and $\mathbb{H}_{F}^{\prime}$ are defined as in Equation 7 Moreover, the cobordism map $\mathfrak{f}_{\mathcal{C}_{\mathcal{F}, \mathfrak{t}}}$ preserves the relative filtration in the sense of Lemma 7.7. In particular, for $\mathbb{M}=\mathbb{F}\left[\mathfrak{u}_{1}, \ldots, \mathfrak{u}_{n}\right]$ which has the structure of an $\mathbb{A}$-module via the homomorphism $\phi: \mathbb{A} \rightarrow \mathbb{M}$ which maps all $\mathfrak{v}_{i}$ to zero, we obtain an invariant homomorphism

$$
\mathfrak{f}_{\mathcal{F}, \mathfrak{t}}=\mathfrak{f}_{\mathcal{C}_{\mathcal{F}, \mathfrak{t}}}^{\mathbb{F}\left[\mathfrak{u}_{1}, \ldots, \mathfrak{u}_{n}\right]}: \operatorname{HFL}^{-}(Y, L, \mathbf{p}, \mathfrak{s}) \rightarrow \operatorname{HFL}^{-}\left(Y^{\prime}, L^{\prime}, \mathbf{p}^{\prime}, \mathfrak{s}^{\prime}\right)
$$




\section{REFERENCES}

[AD] Akram Alishahi and Nathan Dowlin, The Lee spectral sequence, unknotting number, and the knight move conjecture, arXiv:1710.07875.

[AE] Akram Alishahi and Eaman Eftekhary, Knot Floer homology and the unknotting number, in preparation.

[AE15]__ A refinement of sutured floer homology, J. Symplectic Geom. 13 (2015), no. 3, 609-743.

[Ali] Akram Alishahi, The Bar-Natan homology and unknotting number, arXiv:1710.07874.

[Cer70] Jean Cerf, La stratification naturelle des espaces de fonctions différentiables réelles et le théorème de la pseudo-isotopie, Inst. Hautes Études Sci. Publ. Math. (1970), no. 39, 5-173.

[Eft05] Eaman Eftekhary, Longitude Floer homology and the Whitehead double, Algebr. Geom. Topol. 5 (2005), 1389-1418.

[Gab83] David Gabai, Foliations and the topology of 3-manifolds, J. Differential Geom. 18 (1983), no. 3, $445-503$.

[Gab87a] _ Foliations and the topology of 3-manifolds. II, J. Differential Geom. 26 (1987), no. 3, 461-478.

[Gab87b] _ Foliations and the topology of 3-manifolds. III, J. Differential Geom. 26 (1987), no. 3, 479-536.

[GK15] David T. Gay and Robion Kirby, Indefinite Morse 2-functions: broken fibrations and generalizations, Geom. Topol. 19 (2015), no. 5, 2465-2534.

[GW10] J. Elisenda Grigsby and Stephan M. Wehrli, On the colored Jones polynomial, sutured Floer homology, and knot Floer homology, Adv. Math. 223 (2010), no. 6, 2114-2165.

[GWW13] David Gay, Katrin Wehrheim, and Chris Woodward, Connected cerf theory, preprint, 2013.

[JT] András Juhász and Dylan P. Thurston, Naturality and mapping class groups in heegaard floer homology, arXiv:1210.4996.

[Juh] András Juhász, Defining and classifying tqfts via surgery, arXiv:1408.0668.

[Juh06] _ Holomorphic discs and sutured manifolds, Algebr. Geom. Topol. 6 (2006), 1429-1457.

[Juh08] András Juhász, Floer homology and surface decompositions, Geom. Topol. 12 (2008), no. 1, $299-350$.

[Lip06] Robert Lipshitz, A cylindrical reformulation of Heegaard Floer homology, Geom. Topol. 10 (2006), 9551097.

[Mil65] John Milnor, Lectures on the h-cobordism theorem, Notes by L. Siebenmann and J. Sondow, Princeton University Press, Princeton, N.J., 1965.

[OS04a] Peter Ozsváth and Zoltán Szabó, Holomorphic disks and knot invariants, Adv. Math. 186 (2004), no. 1, 58-116.

[OS04b] Holomorphic disks and three-manifold invariants: properties and applications, Ann. of Math. (2) 159 (2004), no. 3, 1159-1245.

[OS04c] Holomorphic disks and topological invariants for closed three-manifolds, Ann. of Math. (2) 159 (2004), no. 3, 1027-1158.

[OS05] _ Heegaard Floer homology and contact structures, Duke Math. J. 129 (2005), no. 1, 39-61.

[OS06] Holomorphic triangles and invariants for smooth four-manifolds, Adv. Math. 202 (2006), no. 2, 326-400.

[OS08] Holomorphic disks, link invariants and the multi-variable Alexander polynomial, Algebr. Geom. Topol. 8 (2008), no. 2, 615-692.

[Ras02] Jacob Andrew Rasmussen, Floer homology of surgeries on two-bridge knots, Algebr. Geom. Topol. 2 (2002), 757-789.

[Zem] Ian Zemke, Link cobordisms and functoriality in link floer homology, arXiv:1610.05207.

Department of Mathematics, Columbia University, New York, NY 10027

E-mail address: alishahi@math.columbia.edu

School of Mathematics, Institute for Research in Fundamental Sciences (IPM), P. O. Box 193955746, TEhran, Iran

E-mail address: eaman@ipm.ir 